\author{
Universidade DE SÃo PAUlO-USP \\ Escola de Engenharia de SÃo Carlos \\ Departamento de Engenharia MecÂnica \\ Programa de Pós-Graduação em Engenharia MecÂnica
}

\title{
Germán Andrés Gaviria Martínez
}

\author{
Towards natural transition in \\ compressible boundary layers
}





\title{
Germán Andrés Gaviria Martínez
}

\section{Em direção a transição em camada limite compressível}

Tese de doutorado apresentada ao Programa de Engenharia Mecânica da Escola de Engenharia de São Carlos como parte dos requisitos para a obtenção do título de Doutor em Ciências.

Área de concentração: Aeronaves

Orientador: Prof. Assoc. Marcello Augusto Faraco de Medeiros Coorientador: Prof. Dr. Elmer Mateus Gennaro

\author{
São Carlos
} aloja o Programa de Pós-Graduação de Engenharia Mecânica. 


\section{AUTORIZO A REPRODUÇÃO TOTAL OU PARCIAL DESTE TRABALHO, POR QUALQUER MEIO CONVENCIONAL OU ELETRÔNICO, PARA FINS DE ESTUDO E PESQUISA, DESDE QUE CITADA A FONTE.}

Gaviria Martínez, Germán Andrés

Em direção a transição em camada limite

GG283e compressível / Germán Andrés Gaviria Martínez;

orientador Marcello Augusto Faraco de Medeiros;

coorientador Elmer Gennaro. São Carlos, 2016.

Tese (Doutorado) - Programa de Pós-Graduação em Engenharia Mecânica e Área de Concentração em Aeronaves - Escola de Engenharia de São Carlos da Universidade de São Paulo, 2016.

1. Transição para turbulência. 2. Pacote de ondas. 3. Camada limite compressível. 4. Instabilidade secundária. 5. Simulação DNS. 6. Transição natural. 7 . Camada limite incompressível. 8. Solução numérica das Equações de Navier-Stokes. I. Título. 


\section{FOLHA DE JULGAMENTO}

Candidato: Físico GERMAN ANDRES GAVIRIA MARTINEZ.

Título da tese: "Em direção a transição em camada limite compressível".

Data da defesa: 02/09/2016.

Comissão Julgadora:

\section{Resultado:}

Prof. Associado Marcello Augusto Faraco de Medeiros Aptosedo (Orientador)

(Escola de Engenharia de São Carlos/EESC)

Prof. Dr. Cristiano Bigonha Tibiriça

(Escola de Engenharia de São Carlos/EESC)

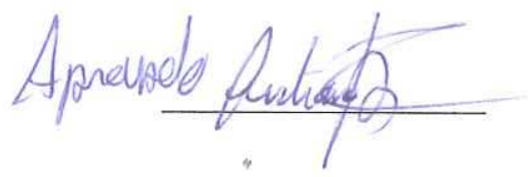

Prof. Dr. Savio Brochini Rodrigues

(Universidade Federal de São Carlos/UFSCar)

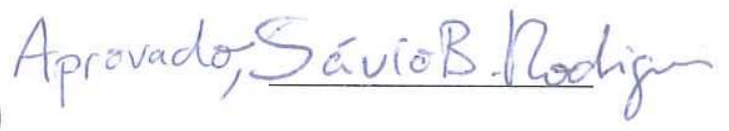

Prof. Dr. Igor Braga de Paula

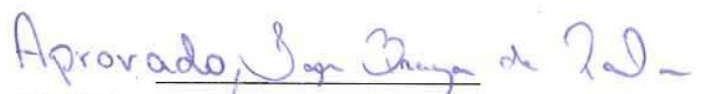

(Pontifícia Universidade Católica do Rio de Janeiro/PUC)

Prof. Titular Gustavo Carlos Buscaglia

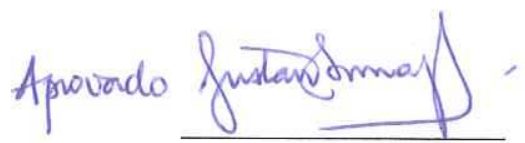

(Instituto de Ciências Matemáticas e de Computação/ICMC-USP)

Coordenador do Programa de Pós-Graduação em Engenheira Mecânica:

Prof. Associado Gherhardt Ribatski

Presidente da Comissão de Pós-Graduação:

Prof. Associado Luis Fernando Costa Alberto 

To my family 



\section{Agradecimentos}

Ao meu orientador Prof. Dr. Marcello Augusto Faraco de Medeiros, pela oportunidade, orientação e pelas experiências compartilhadas no desenvolvimento desde trabalho.

À Escola de Engenharia de São Carlos pelo suporte.

Ao grande número de amigos que encontrei no Brasil, que fizeram da minha estância uma experiência pessoal inestimável.

Ao Conselho Nacional de Desenvolvimento Científico e Tecnológico (CNPQ), pela bolsa de estudos concedida durante o desenvolvimento deste trabalho.

À Air Force Office of Scientific Research (AFOSR), pelo apoio financeiro, através do projeto grant FA9550-11-1-0354-P00002.

A todos que, de alguma forma contribuiram para a realização deste trabalho. 



\section{Resumo}

Gaviria M., G. A. Em direção a transição em camada limite compressível. 141 p. Tese de doutorado - Escola de Engenharia de São Carlos, Universidade de São Paulo, 2016.

No presente trabalho, um código DNS (Direct Numerical Simulation) foi desenvolvido para abordar problemas de transição para tubulência em camada limite subsônica compressível em uma placa plana. Foram realizados testes de validação de código, nos regimes linear e não linear do processo de transição, nos regimes incompressível e compressível. O foco do presente trabalho é estudar transição natural modelada por meio de pacotes de onda em camada limite compressível subsônica, e realizar uma análise preliminar da transição induzida por ruído branco.

Três assuntos principais foram considerados: uma simulação DNS e uma análise comparativa com o experimento (MEDEIROS; GASTER, 1999b) sobre a evolução de um pacote de ondas em camada limite incompressível, a influência da compressibilidade na evolução de pacotes de ondas no regime subsônico, e por último, um estudo preliminar da tranisção induzida por ruído branco em Mach 0.2 e Mach 0.9 .

As comparações realizadas entre a solução numérica e os dados experimentais mostram uma boa concordância, nos regimes linear e não linear, tanto no espaço físico quanto no espaço de Fourier. A simulação numérica deste experimento e a análise realizada neste trabalho, não são encontradas na literatura para o regime incompressível. A análise modal não linear aplicada aos resultados, permitiu identificar a presença das resonâncias tipo H e tipo K no pacote de ondas.

A influência da compressibilidade na evolução dos pacotes de onda foi estudada em Mach 0.7 e Mach 0.9. Na literatura não há trabalhos sobre pacotes de ondas no regime subsônico. No regime linear da transição, os modos oblíquos resultam ser os mais instáveis para Mach > 0.7, como era de esperar, de acordo com os resultados da literatura. No regime não linear, foram observadas estrias de moderada amplitude, associadas com mo- 
dos de baixa frequência que acabam decaindo. O pacote de ondas em Mach 0.9 apresentou amplificação não linear somente na banda subharmônica, que pode ser associada com transição tipo H ou resonância dessintonizada. No entanto, o comportamento geral neste regime é estabilizante. Por sua vez, a interação entre pacotes de ondas em Mach 0.9 mostrou um comportamento desestabilizante, pois a interação acaba gerando amplificação não linear em modos que decaem no pacote isolado. Os modos amplificados sugerem a presença do mecanismo de transição oblíqua.

Finalmente, a evolução da mesma perturbação constituída por ruído branco em Mach 0.2 e Mach 0.9, resultaram ser completamente diferentes. Na camada limite incompressível foram observados vórtices tipo lambda, que poderiam ser gerados pela presença localizada das resonâncias tipo H e/ou tipo K. No regime compressível foram observados vórtices distribuidos em todo o domínio, o que sugere a presença da transição oblíqua. Na transição gerada por ruído branco a compressibilidade teve uma influência maior que no pacote de ondas. Nas condições estudadas, a interação entre pacotes de ondas parece ser uma melhor representação do ruído branco no regime compressível.

Palavras-chave: Camada limite incompressível, camada limite compressível, pacote de ondas, transição natural, simulação DNS, instabilidade secundária, transição para turbulência. 


\section{Abstract}

Gaviria M., G. A. Towards natural transition in compressible boundary layers. 141 p. Ph.D. Thesis - São Carlos School of Engineering, University of São Paulo, 2016.

In this work, a DNS code was developed to investigate problems on transition in subsonic compressible boundary layer on a flat plate. Code validation tests were performed for linear and nonlinear stages of transition, on incompressible and compressible regimes. The focus of the present work is to investigate natural transition in subsonic boundary layers modeled by wave packets; and perform a preliminary study of transition induced by white noise.

Three main problems were considered, namely, a DNS simulation and analysis of the experiment (MEDEIROS; GASTER, 1999b) of wave packet evolution on incompressible boundary layer, the influence of compressibility on wave packet evolution at subsonic Mach numbers and finally, a preliminary study of the evolution of a white noise perturbation in the boundary layer at Mach 0.2 and Mach 0.9.

Comparisons between numerical and experimental results show remarkably good agreement in the linear and nonlinear stages, in both, spatial and Fourier spaces. A numerical simulation of this experiment and the analysis carried out is not available in the literature for wave packets in the incompressible boundary layer. The nonlinear modal analysis performed established the existence of tuned fundamental and subharmonic resonance of H-type and K-type in the packet.

Influence of compressibility in the wave packet evolution was here investigated in boundary layers at Mach 0.7 and Mach 0.9. There are no works reported in the literature on wave packets in compressible subsonic boundary layer. In the linear regime, the oblique modes were the most unstable for Mach $>0.7$, as expected by the results of the literature. In the nonlinear regime, strong streaks were observed, associated with low frequency modes that eventually decay downstream. An isolated wave packet at Mach 0.9 showed 
nonlinear amplification only in the subharmonic band, which may be associated to H-type or detuned resonance. However this packet has a relatively stable character. On the other hand, at Mach 0.9 spanwise interaction of wave packet pairs were more unstable than the isolated case, because stable modes for the isolated packet evolution becomes unstable in the wave packet interaction. This scenario evidenced the presence of oblique transition.

Finally, the nonlinear evolution of the same white noise disturbance at Mach 0.2 and Mach 0.9 were observed to be completely different. In the incompressible boundary layer localized lambda vortex structures were observed, that could be associated to the local presence of H-type and/or K-type resonance. In the compressible regime, longitudinal vortex structures distributed across the entire domain seemed to be linked to oblique transition. In the white noise evolution, compressibility seems to have a stronger effect than in the wave packet evolution. In the conditions considered, the wave packet interaction appear to be a better representation of white noise compressible transition scenario.

Keywords: Incompressible boundary layer, wave packet, natural transition, compressible boundary layer, DNS simulation, secondary instability, transition to turbulence. 


\section{List of Figures}

Figure 1.1 Paths to turbulence in boundary layers. (Reproduced from (FEDOROV, 2011)) . . . . . . . . . . . . . . . . . 29

Figure 1.2 Stages of the transition process in the boundary layer. 1) Laminar flow 2) Primary instability 3) Secondary instability 4) Highly nonlinear region with presence of turbulent spots 5) Fully turbulent flow. The region of interest in the present work is indicated by the red line.

Figure 1.3 Tollmien-Schlichting wave in the diagram instability. Reynolds number is based on boundary layer displacement $\delta^{*}(x)$ and non-dimensional frequency. . . . . . . . . . . . . . . . . . . 3

Figure 2.1 Modified wave number for several schemes. (a) 5 points stencil for $w_{1}=1.5 ; w_{1}=1.8 ; w_{1}=2.2$ and $w_{1}=2.4$. (b) 7 points stencil to $w_{1}=2.3, w_{2}=2.4 ; w_{1}=2.4, w_{2}=2.5 ; w_{1}=2.5, w_{2}=2.6$. Comparison with tridiagonal sixth order scheme. (Reproduced from (BERGAMO, 2013)) . . . . . . . . . . . . . . . . . . 42

Figure 2.2 Phase velocity for several schemes. (a) 5 points stencil for $w_{1}=1.5$; $w_{1}=1.8 ; w_{1}=2.2$ and $w_{1}=2.4$. (b) 7 points stencil to $w_{1}=2.3$, $w_{2}=2.4 ; w_{1}=2.4, w_{2}=2.5 ; w_{1}=2.5, w_{2}=2.6$. Comparison with tridiagonal sixth order scheme. (Reproduced from (BERGAMO, 2013)).

Figure 2.3 (a) Grid stretching in the wall normal direction, (b) stretching function and (c) cell size variation.

Figure 2.4 Reflecting outflow boundary conditions applied to the Poiseuille flow in a two-dimensional domain. (a) Reference case (Reproduced from (POINSOT; LELE, 1992)) and (b) DNS simulation are in a very good agreement. . . . . . . . . . . . . . . . . 45 
Figure 2.5 Non-reflecting outflow boundary conditions applied to the Poiseuille flow in a two-dimensional domain. (a) Reference case (Reproduced from (POINSOT; LELE, 1992)) and (b) DNS simulation are in a very good agreement. . . . . . . . . . . . . . 46

Figure 2.6 Steady state boundary layer profile generation. (a) Upstream of the leading edge of the flat plate a free-slip wall is included to avoid nonphysical gradients associated with the plate leading edge. (b) Boundary layer profile. (Figure is stretched in y direction to facilitate visualization) $0.6 \delta^{*}(-), 1.1 \delta^{*}(-.-)$ and $\delta_{99}(\ldots) \ldots \ldots \ldots 4 \ldots \ldots$

Figure 2.7 Moving frame 3D . . . . . . . . . . . . . . . . . . . . . . 48

Figure 2.8 (a) Slab (b) pencil decomposition. (Reproduced from (2DECOMP\&FFT,

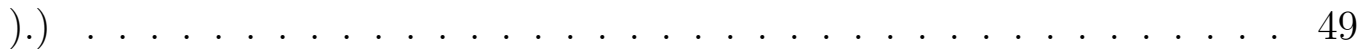

Figure 2.9 Execution diagram and computational cost obtained with gprof. . . . . 50

Figure 2.10 Information of the computational cost obtained with gprof. . . . . . . . 50

Figure 2.11 Speedup of the DNS code. . . . . . . . . . . . . . . 51

Figure 2.12 Diagrams of profiling obtained with vampir, showing detailed information of the communication operations required for parallelization. . . . 52

Figure 3.1 Comparison of Blasius profiles for velocity components, $u$, $v$, with Stewartson solution and DNS results. Comparison of asymptotic values for $\mathrm{v}$ are in a very good agreement.

Figure 3.2 Temperature profile for adiabatic wall and comparison of $T_{a d}$ values calculated from DNS, Stewartson solution and theoretical value (equation 43) at Mach 0.9. . . . . . . . . . . . . . . . . . . . . 55

Figure 3.3 Tollmien-Schlichting amplification curves for incompressible boundary layer. (a) Reproduced from (KONZELMANN; FASEL, 1991, pag. 331) and (b) DNS simulation. . . . . . . . . . . . . . . 56

Figure 3.4 DNS simulation of Fundamental resonance. (a) Comparison of amplification curves with (RIST; FASEL, 1995), (b) Vortical structures generated by the K-type resonance are aligned. (c) Spectrum at Re/1.7208= 460 at $y=0.6 \delta^{*}(x) \ldots \ldots \ldots \ldots \ldots$. . . . . . . . . . . 57

Figure 3.5 Comparison of Subharmonic resonance from (KACHANOV; LEVCHENKO, 1984) and DNS simulation. (a) Amplification curves, (b) staggered vortex pattern and (c) spectral content. . . . . . . . . . . . . 58

Figure 3.6 Comparison of fundamental and subharmonic waves reported in (MASAD; NAYFEH, 1991) and calculated with DNS.

Figure 3.7 Comparison of amplification curves (a) for oblique transition reported in (JOSLIN; STREETT; CHANG, 1993) and calculated with DNS. (b) Streaks in xz plane $(\mathrm{c})$ spectrum $\left(k \beta_{0}, n \omega_{0}\right)$ at $R e=1300$. 
Figure 4.1 Perturbation composition, (a) two-dimensional spectrum, (b) format in streamwise direction, (c) and (d) time signal and time spectral composition. Spanwise perturbation shape in physical space (e) and spectrum (f).

Figure 4.2 Perturbation format in $\mathrm{x}-\mathrm{Z}$ plane. . . . . . . . . . . . . . . . 65

Figure 4.3 Results for grid independence in (a) streamwise and (b) spanwise directions in the nonlinear regime of the wave packet.

Figure 4.4 Test for grid independence in y-direction for a TS-wave in the computational setup for the experiment simulation, with $\omega=0.075$.

Figure 4.5 Grid independence tests in the spanwise direction for an oblique wave defined by $\omega=0.0847$ and $\beta=0.2$. The minimum resolution required in $\mathrm{z}$-direction is 4 points per period of a wave with wavelenght $\lambda_{z}=\frac{2 \pi}{\beta}$.

Figure 4.6 Comparison of velocity time signal at the centerline, at all experimental measurement points of (MEDEIROS; GASTER, 1999a) with DNS simu-

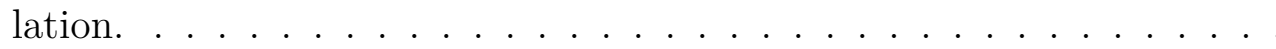

Figure 4.7 Linear wave packet at Mach 0.2 , in $y=0.6 \delta^{*}(x)$. Contour levels at $90 \%(-), 50 \%(-.-), 30 \%(\ldots)$ and $10 \%(--)$ of the amplitude peaks in the packet.

Figure 4.8 Nonlinear wave packet evolution at Mach 0.2 , at $y=0.6 \delta^{*}(x)$. Contour levels at $90 \%(-), 50 \%(-.-), 30 \%(\ldots)$ and $10 \%(--)$ of the amplitude peaks in the packet. . . . . . . . . . . . . . . . . 70

Figure 4.9 Comparison of linear (a) and nonlinear (b) wave packet at advanced position at $y=0.6 \delta^{*}(x)$. Contour levels at $90 \%(-), 50 \%(-.-), 30 \%$ (...) and $10 \%(--)$ of the amplitude peaks in the packet. . . . . . . . 71

Figure 4.10 Comparison of spectral evolution at several measurement points for (a) linear DNS (b) nonlinear DNS and (c) experimental wave packet.

Figure 4.11 Spectrum evolution of the linear wave packet in global nondimensional variables. The frequency of the most amplified mode decays in downstream.

Figure 4.12 Comparison of contour levels of linear, DNS and experimental spectrum in the nonlinear position, $x^{*}=1200 \mathrm{~mm}$.

Figure 4.13 Comparison of $u^{\prime}$ component of the disturbance velocity in the experimental domain at $x^{*}=1300 \mathrm{~mm}, y=0.6 \delta^{*}(x)$. (a) DNS results interpolated at the experimental domain, (b) Experimental results. . . 74

Figure 4.14 Definition of mode bands used for the analysis. (1) linear (2) fundamental (3) subharmonic and (4) low frequency bands.

Figure 4.15 Amplification curves for subharmonic bands of linear (green lines), nonlinear (red lines) and fundamental (blue lines) modes. The evolution of the mode indicated by the red point is shown in the figure 4.16 . 
Figure 4.16 (a) Phase, (b) streamwise wave number, (c) phase velocity for subharmonic resonance, (d) zoom of (c), for the mode $\omega / 2=0.059260$, $\beta=-0.2218$, chosen from experimental spectrum at $x^{*}=1300 \mathrm{~mm} . \quad .76$

Figure 4.17 Amplification curves for the subharmonic mode $(25,15)$ and its fundamental mode. The threshold amplitude of the driving mode $(1,0)$ (indicated by the gray dashed lines) catalyses the subharmonic amplification by increasing the growth rate. The nonlinear growth rate for the mode $(1 / 2,1)$ calculated from H-type controlled transition, matches with the growth rate for the same mode in the packet. . . . . . . . . 79

Figure 4.18 Amplification curves of the most amplified subharmonic in the experimental domain at $x^{*}=1200 \mathrm{~mm} . \ldots \ldots$. . . . . . . . . . 79

Figure 4.19 Analysis of the effective amplitude of the 2D wave driving the H-type instability in the packet. (a)Scaled linear packet. (b) Nonlinear packet. 80

Figure 4.20 Subharmonic most amplified mode at several streamwise positions between $x^{*}=1000 \mathrm{~mm}$ and $x^{*}=1300 \mathrm{~mm}$. . . . . . . . . . . 81

Figure 4.21 Amplification curves for the most amplified subharmonic modes denotes as $(\mathrm{n}, \mathrm{k})$. (a) $(29,14)_{x=1000 \mathrm{~mm}}$, (b) $(24,13)_{x=1100 \mathrm{~mm}}$, (c) $(21,12)_{x=1200 \mathrm{~mm}}$, (d) $(19,11)_{x=1300 \mathrm{~mm}} \ldots \ldots \ldots \ldots$. . . . . . . . . . . . . . . 82

Figure 4.22 The nonlinear amplification of subharmonic modes near to the peak in the subharmonic band, at $x^{*}=1000 \mathrm{~mm}$, can be reproduced closely with H-type controlled transition. (a) 25, 15, (b) 27, 14 . . . . . . . . 82

Figure 4.23 Effect of subharmonic nonlinearity on wave packet shape. . . . . . . . . 83

Figure 4.24 Spectrum of the perturbation with subharmonic band removed. . . . . 84

Figure 4.25 Comparison of spectrum levels at $\mathrm{x}=1300 \mathrm{~mm}$, to investigate subharmonic seeding.

Figure 4.26 Amplification curves and spectrum of the wave packet generated with subharmonic band removed from the perturbation spectra.

Figure 4.27 Amplification curve of the mode $(24,13)$ of the subharmonic mode generated in the reduced disturbance spectrum. . . . . . . . . . . . . 85

Figure 4.28 Amplification curves for fundamental bands of linear (green lines), nonlinear (red lines) and fundamental (blue lines) modes.

Figure 4.29 Mode of the first band, $n=32, k=25$, (a) Phase, (b) streamwise wave number, (c) locking in phase velocity for fundamental resonance. . . . . 87

Figure 4.30 Mode of the second band $2, n=40, k=25$, (a) Phase, (b) streamwise wave number, (c) locking in phase velocity for fundamental resonance.

Figure 4.31 Amplification curves of the most amplified mode in the fundamental band of the experimental spectrum. . . . . . . . . . . . 88

Figure 4.32 Effective amplitude for K-type breakdown. . . . . . . . . . . . . . . 88 
Figure 4.33 Experimental pressure gradient curve. (Reproduced from (MEDEIROS; GASTER, 1999b).)

Figure 4.34 Amplification curves and spectrum at $x^{*}=1200 \mathrm{~mm}$, for a wave packet with the experimental pressure gradient, (a) subharmonic and (b) fundamental mode.

Figure 4.35 Amplification curves and spectrum at $x^{*}=1200 \mathrm{~mm}$, for wave packet with experimental pressure gradient and spectrum calibration at position $x=600 \mathrm{~mm}$. (a) Subharmonic and (b) fundamental mode.

Figure 4.36 1) Higher levels of the disturbance amplitude are linearly related to higher levels of the fundamental modes. Reproduced from (OPFER, 2002, figures 7.10 and 7.11). . . . . . . . . . . . . . . . 92

Figure 4.37 Amplification curves and spectrum for a wave packet with disturbance amplitude increased 20\%. (a) subharmonic and (b) fundamental mode. 92

Figure 4.38 Spanwise modes for $\omega=0.09075$ at $x=1300 \mathrm{~mm}$. . . . . . . . . . . 93

Figure 5.1 N-factor curves for Mach 0.7. The red line indicates the mode 2D most amplified, which in this case corresponds to $\omega=0.0486 \ldots$. . . . . . 97

Figure 5.2 Variation of the frequency of most amplified mode with Mach number. From Mach 0.8 , oblique modes around $\beta \sim 0.07$ are the linearly most unstable. . . . . . . . . . . . . . . . . . 97

Figure 5.3 (a)Linear and (c) nonlinear wave packet at Mach 0.7, in $y=0.6 \delta^{*}(x)$. Contour levels at $90 \%(-), 50 \%(-.-), 30 \%(\ldots)$ and $10 \%(--)$ of the amplitude peaks.

Figure 5.4 Spectral evolution at Mach 0.7, for linear and nonlinear wave packet, with $a_{1}=3.5 \times 10^{-5} \ldots \ldots \ldots \ldots 9 . \ldots \ldots$

Figure 5.5 Spectrum levels for Mach 0.7 at $\mathrm{x}=972 . \quad \ldots \ldots$. . . . . . . . . . 100

Figure 5.6 Amplification curves for Mach 0.7 for selected modes in the spectrum at the position $x=972$. All bands shows strong nonlinear amplification.

Figure 5.7 Spectral evolution for Mach 0.7, for nonlinear wave packet with disturbance amplitude $a_{2}=2.5 * 10^{-5} \ldots \ldots \ldots \ldots 1$

Figure 5.8 Amplification curves for disturbance with $a_{2}=2.5 * 10^{-5}$. . . . . . . 102

Figure $5.9 \mathrm{u}$ component of the velocity at the centerline. Amplitude effect on the nonlinear wave packet evolution at Mach 0.7, (a) linear wave packet, (b) $a_{2}=2.5 \times 10^{-5}$ and (c) $a_{1}=3.5 \times 10^{-5}$

Figure 5.10 Spectrum at the position $x=972$, for the wave packet generated with amplitude $a_{1}=3.5 * 10^{-5}$. (a) Most amplified subharmonic mode (b) non identified resonance, could be a detuned resonance.

Figure 5.11 (a) The oblique mode $(1,1)$ does not resonates with the fundamental mode $(1,1)$. (b) Non identified resonance. $a_{2}=3.5 \times 10^{-5}$. . . . . . 104 
Figure 5.12 Comparison of linear wave packet for (a) Mach 0.2 and (b) Mach 0.9 at the centerline. . . . . . . . . . . . . . . 105

Figure 5.13 Linear wavepacket at Mach 0.9 , in $y=0.6 \delta^{*}(x)$. Contour levels at $90 \%$ $(-), 50 \%(-.-), 30 \%(\ldots)$ and $10 \%(--)$ of the amplitude peaks in the packet. . . . . . . . . . . . . . . . 105

Figure 5.14 Comparison of linear and nonlinear spectrum evolution at Mach 0.9 . 106

Figure 5.15 Nonlinear wavepacket at Mach 0.9, in $y=0.6 \delta^{*}(x)$. Contour levels at $90 \%(-), 50 \%(-.-), 30 \%(\ldots)$ and $10 \%(--)$ of the amplitude peaks in the packet. . . . . . . . . . . . . . . . 108

Figure 5.16 Linear wave packet in incompressible boundary layer $($ Mach $=0.2)$ (a) and (b) at Mach 0.9. (c) Nonlinear wavepacket at Mach 0.9, in $y=0.6 \delta^{*}(x)$. Contour levels at $90 \%(-), 50 \%\left(\right.$-.- $\left.^{-}\right), 30 \%(\ldots)$ and $10 \%(--)$ of the amplitude peaks in the packet. . . . . . . . 108

Figure 5.17 Comparison of linear and nonlinear spectrum levels at Mach 0.9 in $\mathrm{x}=1132 \ldots \ldots \ldots \ldots$

Figure 5.18 Amplification curves at $\mathrm{x}=1132$. Streaks are dominant from the beginning, but eventually decay and reach the linear growth rate. Subharmonic mode remains amplifying nonlinearly. . . . . . . . . . . . . . . . 109

Figure 5.19 Spectrum evolution of nonlinear wave packet at Mach 0.9 in extended domain in streamwise direction, with $x_{\max }=2500$. . . . . . . . . 110

Figure 5.20 Amplification curve at $\mathrm{x}=2412$. Streaks are dominant but decay in downstream. . . . . . . . . . . . . . . . . . 111

Figure 5.21 Phase locking for subharmonic mode. . . . . . . . . . . . . . . . 111

Figure 5.22 Linear evolution in physical of a pair of wave packets separated by $d=30.113$

Figure 5.23 Nonlinear evolution in physical space of a pair of wave packets separated

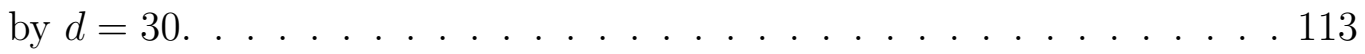

Figure 5.24 Comparison of linear and nonlinear spectral evolution for two packets separated $d=30 \ldots \ldots \ldots \ldots$. . . . . . . . . . . . . . . . . . . . . . . .

Figure 5.25 Comparison of spectrum contour levels at position $x=1132$. . . . . . 115

Figure 5.26 Amplification curves for a pair of wave packets separated 30 units. . . . 115

Figure 5.27 Linear evolution of a pair of wave packets separated by $d=60$. . . . . 117

Figure 5.28 Nonlinear evolution of a pair of wave packets separated by $d=60$. . . 117

Figure 5.29 Wave packet interaction, with distances between center packet (a) $d=$ 30, (b) $d=60$, (c) isolated packet. . . . . . . . . . . . . 118

Figure 5.30 Linear and nonlinear spectral evolution for a pair of wave packets sep-

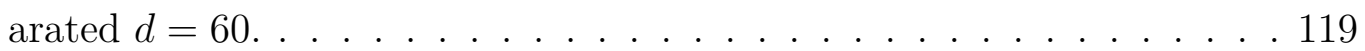

Figure 5.31 Comparison of spectrum levels for a pair of linear and nonlinear wave packets separated $d=60$ at $\mathrm{x}=1132$. . . . . . . . . . . 120

Figure 5.32 Amplification curves for a pair of wave packets separated $d=60$. . . . 120 
Figure 6.1 Temporal and spatial disturbances used to generate white noise perturbation. . . . . . . . . . . . . . . . . . 122

Figure 6.2 (a) Linear and (b) nonlinear evolution at Mach 0.2 for white noise disturbance. Lambda vortex are generated in nonlinear case. . . . . . . 123

Figure 6.3 (a) Linear and (b) nonlinear evolution of white noise at Mach 0.9. Streaks along entire domain are generated. . . . . . . . . . . . . 123

Figure 6.4 Linear and nonlinear evolution of spectral content for white noise perturbation at Mach 0.2. . . . . . . . . . . . . . . . . . . . 124

Figure 6.5 Low frequency modes (most amplified) in white noise transition at Mach 0.2. . . . . . . . . . . . . . . . . . . . . . 125

Figure 6.6 Comparison of spectral levels at $\mathrm{x}=1192$. Low frequency modes are amplified nonlinearly. . . . . . . . . . . . . . . . . . 125

Figure 6.7 Evolution of a white noise perturbation at Mach 0.9 . . . . . . . . . 127

Figure 6.8 Most amplified modes in nonlinear amplification for white noise perturbation at Mach 0.9 . . . . . . . . . . . . . . . . . . . . . . 127

Figure 6.9 Comparison of spectral levels at $\mathrm{x}=796 \ldots$. . . . . . . . . . . . . 128

Figure 6.10 (a) Velocity time signal and Gaussian windowing (b) Fourier transform 128 



\section{List of Tables}

Table 1 Experimental Parameters . . . . . . . . . . . . . . . . . 63 

Nomenclature

LST Linear Stability Theory

DNS Direct Numerical Simulation

TS Tollmien-Schlichting

PSE Parabolized Stability Equations 



\section{Contents}

1 Introduction $\quad 27$

1.1 Relevance . . . . . . . . . . . . . . . . . . . . . . 28

1.2 Transition routes . . . . . . . . . . . . . . . . . . . . . . . . . 29

1.3 Wave packets and natural transition . . . . . . . . . . . . . . 34

1.3.1 DNS simulations of wave packets . . . . . . . . . . . . . . 35

1.4 Objectives of this work . . . . . . . . . . . . . . 36

1.5 Thesis outline . . . . . . . . . . . . . . . 37

$2 \quad$ Physical problem and computational setup $\quad 39$

2.1 Governing equations . . . . . . . . . . . . . . . . . . . . . . . . . . . 39

2.2 Numerical Methods . . . . . . . . . . . . . . . . . . . . . . . 40

2.2.1 Discretization . . . . . . . . . . . . . . . . 40

2.2.2 Compact finite differences . . . . . . . . . . . . . . . . 41

2.2.3 Numerical Stability . . . . . . . . . . . . . . . . . 43

2.2 .4 Filter . . . . . . . . . . . . . . . . . . . 43

2.2.5 Grid stretching . . . . . . . . . . . . . . . . . . . 44

2.2.6 Boundary Conditions . . . . . . . . . . . . . . . . . . 44

2.2.7 3D Moving Frame . . . . . . . . . . . . . . . . . 47

2.3 Parallelization . . . . . . . . . . . . . . . . . . . . . 48

2.3.1 Performance estimation . . . . . . . . . . . . . . . . . . . . 49

3 Code validation $\quad 53$

3.1 Base flow generation . . . . . . . . . . . . . . . . 53

3.1.1 Boundary layer profiles . . . . . . . . . . . . . . 53

3.2 Hydrodynamic instability tests . . . . . . . . . . . . . . . . 56

4 DNS simulation of experimental wave packet in an incompressible $\begin{array}{ll}\text { boundary layer } & 61\end{array}$ 
4.1 Numerical set-up . . . . . . . . . . . . . . . . . . . 6 61

4.1.1 Choice of reference experiment . . . . . . . . . . . . . 61

4.2 Disturbance Generation . . . . . . . . . . . . . . . . . . . 63

4.2 .1 Grid independence tests . . . . . . . . . . . . 66

4.3 DNS results . . . . . . . . . . . . . . . . . . . . . . . . . 68

4.3 .1 Linear wave packet . . . . . . . . . . . . . . . 68

4.3.2 Comparison of numerical with experimental wave packet . . . . . . 68

4.4 Nonlinear modal analysis of DNS results . . . . . . . . . . . . . 75

4.4 Subharmonic bands . . . . . . . . . . . . . . . . . . . . . 75

4.4.2 Asymmetry generated by the nonlinear effects . . . . . . . . . . 83

4.4.3 Subharmonic seed . . . . . . . . . . . . . . . . 83

4.4 .4 Fundamental bands . . . . . . . . . . . . . . . . 86

4.5 Effective experimental conditions . . . . . . . . . . . . . . . . . . . . 89

4.5.1 Pressure gradient . . . . . . . . . . . . . . . . . . 89

4.5.2 Amplitude calibration . . . . . . . . . . . . . . . . 90

4.5.3 Amplitude effect . . . . . . . . . . . . . . . . . . 91

$5 \quad$ Wave packet in compressible boundary layer $\quad 95$

5.1 Numerical set-up . . . . . . . . . . . . . . . . . . . . 95

5.2 Wave packet in a boundary layer at Mach $0.7 \ldots \ldots$. . . . . . . . 96

5.2 .1 Linear wave packet . . . . . . . . . . . . . . 96

5.2 .2 Nonlinear wave packet . . . . . . . . . . . . . . . 98

5.2 .3 Amplitude effect . . . . . . . . . . . . . . . . . . . . 101

5.2.4 Analysis of possible secondary instability regime . . . . . . . . . 103

5.3 Wave packet in a boundary layer at Mach 0.9 . . . . . . . . . . . . . . 104

5.3 .1 Linear wave packet . . . . . . . . . . . . . . . . . . . . 104

5.3.2 Nonlinear wave packet . . . . . . . . . . . . . . 107

5.4 Interaction between wavepackets in a boundary layer at Mach 0.9 . . . . 112

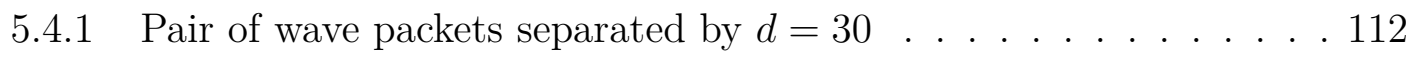

5.4 .2 Pair of wave packets separated by $d=60 \ldots 116$

6 Transition generated by white noise 121

6.1 Generation of white noise disturbance . . . . . . . . . . . . . . . . . . 121

6.2 White noise evolution at Mach $0.2 \ldots$. . . . . . . . . . . . . . . . . 122

6.3 White noise evolution at Mach $0.9 \ldots \ldots$. . . . . . . . . . 126

$\begin{array}{llr}7 & \text { Conclusions and remarks } & 129\end{array}$

7.1 Conclusions . . . . . . . . . . . . . . . . . . . . . . . 129

$\begin{array}{ll}\text { Bibliography } & 133\end{array}$ 


\section{Introduction}

Natural transition is the term coined in literature (KACHANOV, 1994; PAULA et al., 2013) to refer to the transition to turbulence process as it occurs in real (uncontrolled) conditions, generated by perturbations with random spectral content and moderate amplitudes, $<2 \%$ of the free-stream velocity as was observed experimentally by Peltzer (2008) and Reeh e Tropea (2015). Transition to turbulence in boundary layers is triggered by infinitesimal perturbations that begin to be amplified by the flow from the critical Reynolds number $R e_{c r}$ up to the transition point $R_{T}$, which indicates the turbulence onset. In the transition region, the flow acts as a band-pass filter, amplifying through linear and nonlinear mechanisms the unstable bands of modes. Temporal and three-dimensional complex variations on the flow are observed, as was firstly evidenced in the pioneer experiment (REYNOLDS, 1883). In real conditions, disturbances in the form of thermal, mechanical and acoustic perturbations, surface irregularities, free stream turbulence, among others, cannot be completely removed, and always are present influencing the flow. The transition process is very sensitive to flow characteristics and disturbance parameters, such as amplitude, spectrum and source of perturbation, (SINGER; FERZIGER; REED, 1986). It is a very intermittent phenomenon, difficult to measure and reproduce.

Given the complexity of the problem, investigations on natural transition consider several simplified approaches. For example, two-dimensional waves interacting with a low amplitude band of three-dimensional modes (SPALART; YANG, 1987; FASEL, 2002; BORODULIN; KACHANOV; KOPTSEV, 2002), transition induced by free-stream turbulence (BRANDT; SCHLATTER; HENNINGSON, 2004; RICCO; WU, 2007), white noise (YANG; SPALART; FERZIGER, 1992; SHAIKH, 1997) and wave packets, which are the focus of this work.

The use of wave packets as a model for natural transition is justified by their broadband spectra and by experimental observations, where wave packets have been identified in the time-velocity signals induced by free stream turbulence (SCHUBAUER; SKRAMSTAD, 1943; GASTER, 1993) and by artificially excited white noise (SHAIKH, 1997). Through Fourier analysis, individual modes in the wave packet can be monitored to identify linear and nonlinear interactions, and the results can be related with observations in natural 
transition.

\section{$1.1 \quad$ Relevance}

In many practical situations prediction or control of the transition point is crucial, as well as the determination of flow properties in the transition regime. In some cases, it is needed to promote turbulence as in chemical mixing, combustion or to avoid separation. Delay of turbulence onset is important for the extension of laminar flow, because it is related with lower drag and higher lift coefficients. Investigations on a Airbus A320, (MAREC, 2001), evidence that skin friction drag is responsible for about $50 \%$ of the total drag, from this percentage $25 \%$ is generated on the wings. Depending on the specific part of the airplane, it is possible to achieve different levels of drag reduction. An extreme case is the drag of the fin, that can be reduced in $38 \%$ resulting in a saving of $1.3 \%$ in fuel consumption. In subsonic aircraft, fuel represents around $27 \%$ of DOC (Direct Operational Cost), in supersonic aircraft, it represents $35 \%$.

The laminar region can be extended using flow control systems or by wing shape optimizations. High cost and technical requirements of the control systems, suggest that drag reduction based on laminar wings would give more benefits. However, design of laminar wings is a challenge, because limitations in understanding the transition process difficult refined optimizations. Low error drag estimations depend on practical and accurate methods, which reduce time in design cycles.

The dimensionless drag count coefficient is a measure of drag. At cruise, the drag count varies between 200 to 400. For subsonic cruise 1 drag count is equivalent to $100 \mathrm{~kg}$. For supersonic cruise $1 \mathrm{drag}$ count is equivalent to $5 \%$ of the payload. The accuracy desired for drag calculation is 10 drag counts for subsonic, 4 for transonic cruise and 1 for supersonic cruise, (THIBERT; ARNAL, 2000). Methods used in aircraft industry can have errors on estimations on drag on the order of $10 \%$, transonic cruise presents even higher deviations (THIBERT; ARNAL, 2000; KAYNAK; GüRDAMAR, 2008). The formulation of accurate and practical methods is based on a detailed comprehension of the transition process that remains as an open problem.

Usually engineering predictions of transition are performed by means of empirical correlations or semi-empirical methods (DINI; SELIG; MAUGHMER, 1992), as for example $e^{N}$ method (INGEN, 2008), which is one of the most employed . These solutions produce acceptable results on engineering applications but do not explain the transition phenomenon. 


\subsection{Transition routes}

Depending mostly on the perturbation amplitude and spectral composition, there are two main routes of transition to turbulence, bypass transition and modal amplification (FEDOROV, 2011). The whole process is shown in the figure 1.1. Bypass transition is induced by high perturbation amplitudes, In this case, the flow changes abruptly from laminar to turbulent, this route is not considered in this work. In modal route, the flow evolves through several stages, as sketched in the figure 1.2, changing flow properties and developing complex vortical structures gradually. The present work focuses in the linear and moderate nonlinear stages of transition, that corresponds to the region indicated by the red line in the figure.

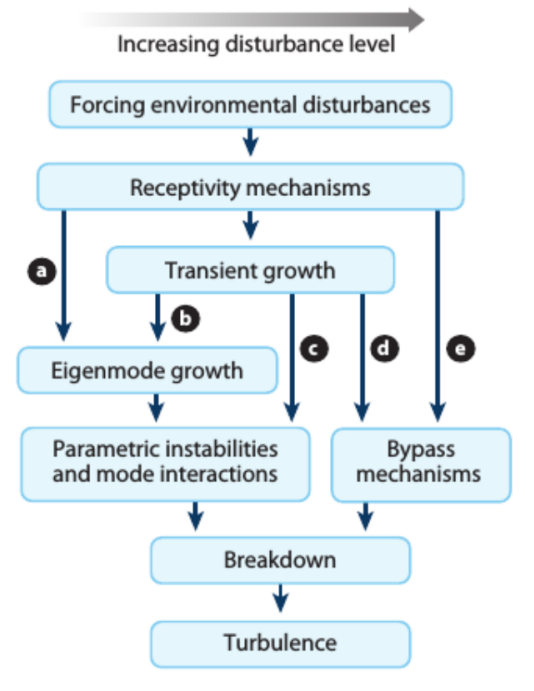

Figure 1.1: Paths to turbulence in boundary layers. (Reproduced from (FEDOROV, 2011)).

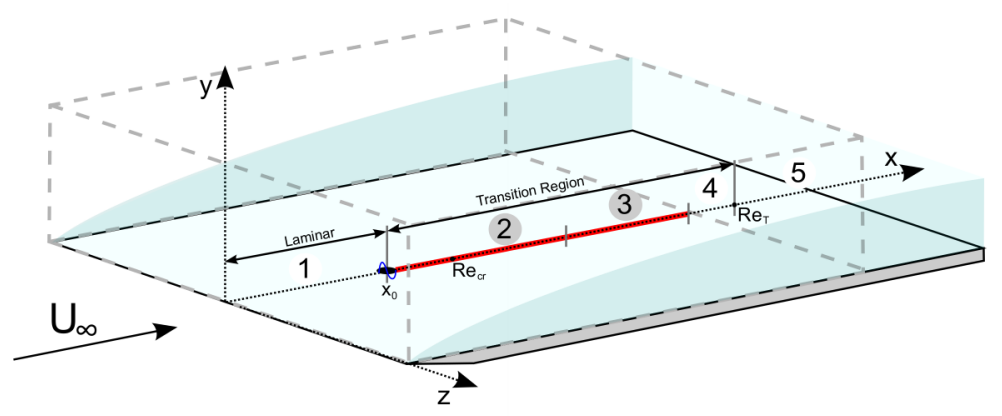

Figure 1.2: Stages of the transition process in the boundary layer. 1) Laminar flow 2) Primary instability 3) Secondary instability 4) Highly nonlinear region with presence of turbulent spots 5) Fully turbulent flow. The region of interest in the present work is indicated by the red line. 
The simplest case of hydrodynamic instability in a boundary layer consists of a monochromatic perturbation with infinitesimal amplitude, relative to the free-stream velocity. Also known as primary instability, this stage is responsible for the generation of the so called Tollmien-Schlichting (TS) waves and it is described by the Linear Stability Theory (LST). Usually, subsonic incompressible regime occupies the larger part of the transition region, generating two-dimensional waves. This theory is derived from the linearization of the Navier Stokes equations, and arrives at the Orr-Sommerfeld equation (CRIMINALE; JACKSON; JOSLIN, 2003; SCHMID; HENNINGSON, 2001). This equation is formulated in terms of the undisturbed or base flow $u_{\text {base }}$, and the disturbance $u^{\prime}$, which results from subtraction of the base flow from the perturbed flow:

$$
u^{\prime}=\bar{u}-u_{\text {base }}
$$

The ansatz for the solution of the Orr-Sommerfeld equation is a harmonic wave described by:

$$
u^{\prime}=\exp (i(\alpha x+\beta z-\omega t))
$$

In the spatial analysis, the frequency $\omega$ and spanwise wave number $\beta=\frac{2 \pi}{\lambda_{z}}$ are real and considered as parameters, then, $\alpha=\alpha_{x}+i \alpha_{i}$ results from the flow response and is an eigenvalue for this problem. Theoretical results of linear instability for boundary layer can be summarized in the instability diagram (figure 1.3). The flow amplifies the unstable band of frequencies, defined by the inner region of the diagram. This corresponds to the Tollmien-Schlichting waves. The points where disturbances start to grow and to decay are know as first and second branch respectively. Their amplitudes increase in downstream up to the end of the unstable region, as sketched in figure 1.3. TS waves were predicted theoretically at the beginning of 1930 decade (TOLLMIEN, 1929; SCHLICHTING, 1933) and confirmed experimentally in the classic experiment of (SCHUBAUER; SKRAMSTAD, 1943) in the first low turbulence wind tunnel. Previous attempts to detect TS waves failed due to relative high turbulence intensity in the wind tunnels. Results only were published after the Second World War. Tollmien-Schlichting waves have a two-dimensional wave front, they are characterized by the growth rate $\alpha_{i}$, streamwise wave number $\alpha_{x}$, propagation angle $\psi$. These waves propagates with phase velocity $c_{p}$. When $\psi \neq 0$, the TollmienSchlichting waves also are known as oblique waves with spanwise number $\beta$. From the flow variables, this parameters can be calculated as:

$$
\psi=\operatorname{atan} \frac{\beta}{\alpha_{x}}, \quad \alpha_{x}=\frac{d \theta(x)}{d x}, \quad \alpha_{i}=-\frac{d[\ln (A(x))]}{d x}, \quad c_{p}=\frac{\omega}{\alpha_{x}}
$$

With $A(x)$ the amplitude of the disturbance, $\theta(x)$ the phase, which is calculated by performing Fourier transform over the time signals in the streamwise velocity component. For an oblique wave with spanwise wave number $\beta$, the phase velocity is given by (KACHANOV; 
LEVCHENKO, 1984):

$$
c_{p}=\frac{\omega \alpha_{x}}{\alpha_{x}^{2}+\beta^{2}}
$$

A mode can be identified by the parameters $(\omega, \beta)$ or in terms of the harmonics $(n, k)$ of a given fundamental mode $\left(\omega_{0}, \beta_{0}\right)$.

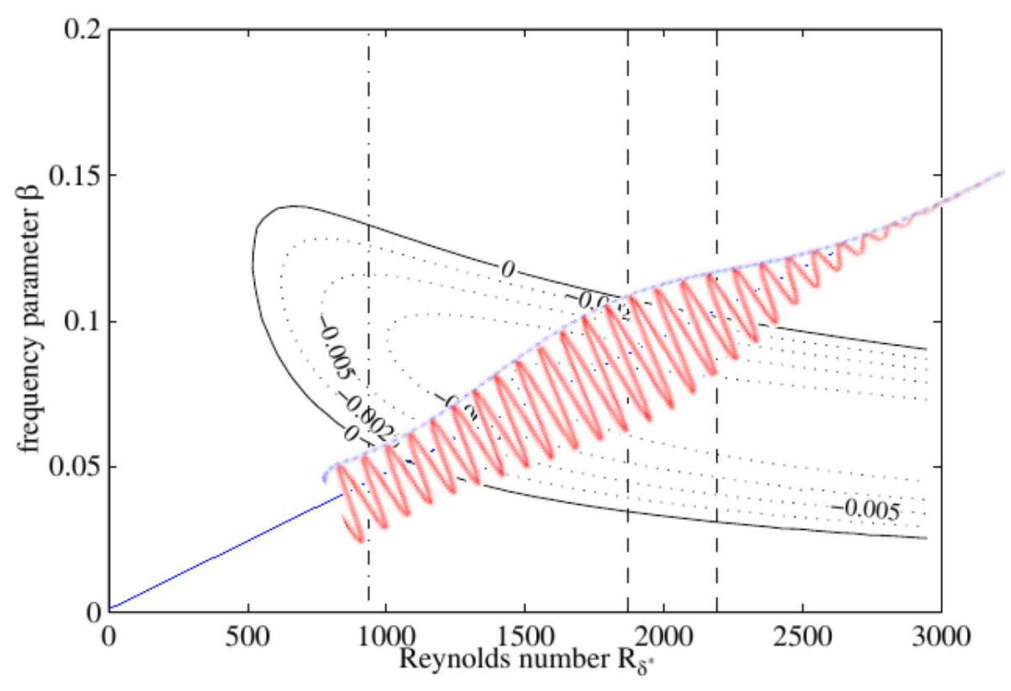

Figure 1.3: Tollmien-Schlichting wave in the diagram instability. Reynolds number is based on boundary layer displacement $\delta^{*}(x)$ and non-dimensional frequency.

At disturbance amplitudes, around $0.02 \%$ to $0.1 \%$ of the free stream velocity, moderate nonlinear effects arise, with strong amplification in a short spatial region. Growth rates increase substantially with respect to the linear growth rates. Three-dimensional vortical structures are generated, with a defined spatial and temporal periodicity. Also skin drag coefficient increases and distortion on the velocity profiles is observed (SAYADI; HAMMAN; MOIN, 2013).

Under controlled conditions, three nonlinear mechanisms of wave growth have been discovered, namely, fundamental or K-type (KLEBANOFF; TIDSTROM; SARGENT, 1962; KACHANOV, 1987), subharmonic or H-type (KACHANOV; KOZLOV; LEVCHENKO, 1977; KACHANOV; LEVCHENKO, 1984), both identified in experimental observations and oblique resonance (O-type) discovered numerically, initially for compressible boundary layer at Mach 1.6 by (BESTEK; THUMM; FASEL, 1993) and for incompressible boundary layer by (SCHMID; HENNINGSON, 1992). Another kind of nonlinear amplification is proposed by (WU; STEWART, 1996; WU; STEWART; COWLEY, 2007), which establishes that nonlinear resonance occurs when waves have the same phase velocity. These kinds of nonlinear amplification are known as secondary instability. These mechanisms separately can lead to a complete breakdown to turbulence (SAYADI; HAMMAN; MOIN, 2013; SIVASUBRAMANIAN; FASEL, 2014). An isolated two-dimensional TS-wave can not promote transition, at least low amplitude oblique waves are needed. It is not clear entirely if these mechanisms 
are present in natural transition, how they coexist or interact or if there are unknown nonlinear mechanisms. These mechanisms are relevant because in more complex cases, such as wave packets, several of their characteristics have been qualitatively observed. For disturbance amplitude larger than $\% 5$ of the free-stream velocity, strong nonlinear effects are dominant, turbulent spots are generated and fully turbulent regime is achieved.

Subharmonic breakdown is triggered by a threshold amplitude of the fundamental mode $(1,0)$, which resonates with the mode $(1 / 2, \pm 1)$. In this mechanism, three-dimensional vortical structures with a staggered pattern are generated. In the region of subharmonic amplification the velocity amplitude varies in the range $0.02 \%$ to $0.06 \%$, influencing the spatial vortex periodicity (BAKE; FERNHOLZ; KACHANOV, 2000).

Fundamental resonance can be initiated by the interaction of a 2D mode (primary wave) $(1,0)$ and a pair of oblique waves $(1, \pm 1)$ with low amplitude or by the interaction of the primary wave and the pair of steady modes $(0, \pm 1)$, (see (BERLIN; WIEGEL; HENNINGSON, 1999)). The primary mode has a catalytic role, because the energy transferred to the oblique waves comes from the base flow, not from the 2D mode, which keeps its energy almost without variation. In this mechanism aligned vortical structures are generated, it develops in a short region and generates peaks in velocity signal. In the process, harmonics in frequency and spanwise modes are generated and amplified. It is more explosive than subharmonic resonance and develops in a shorter region. During the resonance process, both modes reach the same phase velocity. This is known as phase locking. In the subharmonic resonance, threshold amplitude and phase locking have analog behavior as for fundamental resonance.

Oblique transition is generated by the nonlinear interaction of a pair of oblique waves $(1, \pm 1)$. In this mechanism, there is no threshold amplitude. Modes grow linearly and transfer energy to a steady mode $(0, \pm 2)$, while also harmonics $(0,2 k)$ are generated . Linear and nonlinear behavior are difficult to separate. (SCHMID; REDDY; HENNINGSON, 1996) concluded that oblique transition can be triggered by a lower energy in the oblique waves than the energy required by the $2 \mathrm{D}$ waves in H-type and K-type resonances. For high amplitudes of the oblique waves, the oblique transition can induce turbulence, for lower amplitudes all modes eventually decay. Several works, (MAYER; WERNZ; FASEL, 2011; MAYER; TERZI; FASEL, 2011) shows that oblique breakdown is a viable path to turbulence in supersonic boundary layers.

Compressibility can change stability properties of a given flow. Research of transition in compressible boundary layer come after of its incompressible counterpart, owing mainly to experimental difficulties and problem complexity by the increase of variables and parameters. There are only a few experiments of controlled transition on supersonic boundary layers: the experiment of (SEMIONOV; KOSINOV; MASLOV, 1999) for Mach 2 is commonly 
used as main reference for low supersonic Mach numbers. Investigations on nonlinear transition in compressible boundary layers start at the ends of the 1980s, with studies on secondary instability. This coincides with a noticeable improvement in the calculation capacity and the cost reduction of the computational resources. Since then, the amount of numerical investigations is increasing. Also in these years, efficient numerical approaches like the Parabolized Stability Equations (PSE) (BERTOLOTTI; HERBERT; SPALART, 1992) were formulated, which are capable of reproducing moderate nonlinear behavior of the Navier-Stokes equations. These methods were applied to compressible boundary layers to investigate the initial stages of the nonlinear regime of transition (CHANG et al., 1991). In subsequent years, several systematic studies were performed on secondary instability at subsonic (El-Hady Nabil M., 1991; MASAD; NAYFEH, 1991), and supersonic boundary layers (NG; ERLEBACHER, 1992). Also, (THUMM; WOLZ; FASEL, 1990) simulated secondary instability for compressible boundary layer at several Mach numbers. In recent theoretical works, spatial modes (TUMIN, 2007) and the effect of heat transfer on Klebanoff modes (RICCO; TRAN; YE, 2009) are investigated. More recently, a new mode was discovered for compressible boundary layers (TUNNEY et al., 2015).

Compressibility alters stability properties of a given flow. According to LST (MACK, 1975; GAPONOV et al., 1982; MACK, 1987; ÖZGEN; KIRCALI, 2007), at subsonic Mach numbers the unstable region is similar to the incompressible case. The main differences result in a reduction of the growth rates and the critical Reynolds numbers, (ÖZGEN; KIRCALI, 2007). Also, in contrast to incompressible boundary layers, for Mach $\geq 0.7$ the $3 \mathrm{D}$ disturbances with angle of propagation $45^{\circ}<\psi<60^{\circ}$ are more unstable than 2D waves (WHITE, 1974), the effect is strongest in the range $2<\mathrm{Mach}<3$.

At subsonic Mach numbers compressibility has an overall stabilizing effect and, as a direct consequence, the transition region is longer in the nonlinear regime (MARTINEZ; GENNARO; MEDEIROS, 2015). However, the set of most unstable modes can change, depending on the specific value of the Mach number, due to several effects, some of them, described below.

Results on secondary instability mechanisms (El-Hady Nabil M., 1991), showed that for subsonic Mach numbers, the subharmonic resonance has less intensity than the incompressible counterpart, then, it is needed a longer region to develops a nonlinearity of this kind. Fundamental resonance is stronger than subharmonic, and more intense with the propagation angle (THUMM; WOLZ; FASEL, 1990).

In subharmonic resonance there is a preferred band in spanwise wave numbers (NG; ERLEBACHER, 1992). In subsonic boundary layers heat transfer has a stronger effect on linear instability than in supersonic ones (SCHLICHTING; GERSTEN, 2003, pag. 467). By adding heat into the boundary layer, unstable region increases, while heat removal has a stabilizing effect (KAZAKOV; KOGAN; KUPAREV, 1985). Methods for transition prediction used in industry are mainly based on linear amplification and only limited nonlinear 
effects are included, for example, by coupling Navier-Stokes solvers with the $e^{N}$ method (YONGSHENG; WE, 2007), or using nonlinear models of natural transition, (KUROTAKI et al., 2007). Sometimes additional effects, such as the compressibility (KAYNAK; GüRDAMAR, 2008) are also taken into account.

Natural transition develops through different routes depending, mainly, on the specific characteristics of spectral content and amplitude of the perturbation. These routes may include secondary instability mechanisms, that could coexist interchanging dominance along transition stages depending on specific conditions. Probably unknown scenarios are also present, generating intermittency and formation of turbulent spots. Investigation of the aspects are the focus of actual research.

\subsection{Wave packets and natural transition}

Early works on wave packets were experimental, establishing the main characteristics of the packet evolution. (VASUDEVA, 1967) reveals selective nonlinear amplification in the streamwise direction. The following experiment focused on linear evolution (GASTER; GRANT, 1975) to compare results with a linear model (GASTER, 1975). Later, (COHEN; BREUER; HARITONIDIS, 1991) performed measurements of the complete transition process, from linear amplification up to the formation of turbulent spots. In (COHEN, 1994) analysis for individual modes is carried out, to identify nonlinear amplification. Subsequent experiments focused in several aspects of moderate nonlinear regime. (MEDEIROS; GASTER, 1999b) investigated the origin of nonlinearly generated modes, the phase effect of the perturbation (MEDEIROS; GASTER, 1999a) and the effect of magnitude of the modulation (MEDEIROS, 2004). By performing variations on the spectral content of the wave packet disturbance, (BECH; HENNINGSON; HENKES, 1998) observed that the pressure gradient has a large influence on the evolution than the packet shape. In these works, bands of nonlinearly amplified modes suggest the presence of secondary instability mechanisms, both fundamental and subharmonic. Usually, in the experiments and simulations of wave packets, the frequency spectrum of the perturbation is carefully controlled, and less attention is given to the spanwise spectrum.

In the literature, the dominant nonlinear activity in wave packet has been attributed to subharmonic instability of the $\mathrm{C}$ or $\mathrm{H}$ type. Nevertheless, the conclusion is generally based only on the fact that these waves fall close to the subharmonic band, and that the dominant spanwise wave number is close to that obtained for subharmonic resonance for controlled (unmodulated wave) transition (COHEN; BREUER; HARITONIDIS, 1991; MEDEIROS; GASTER, 1999b; SIVASUBRAMANIAN; FASEL, 2014). It is clear in the literature that the dominant subharmonic mode in the packet does not have exactly half the frequency of the dominant primary fundamental mode in the packet. The frequency 
ratio is closer to $2 / 3$ and is often regarded as evidence of detuned subharmonic resonance (BORODULIN et al., 2002). However such resonance would normally be associated with two modes symmetric with respect to the actual subharmonic frequency. This state of affairs led to suggestions that a different mechanism could be at play (YEO et al., 2010), namely phase locking mechanism (WU; STEWART, 1996). It is apparent that the issue is not yet settled. It seems that comparison of actual growth rates would be a much more definite evidence for conclusion, but this was never shown in the literature, except in the experiments of (PAULA et al., 2013), which performed such an analysis for waves modulated only in the streamwise direction. Paula et al. (2013) produced the streamwise modulation by combining 2 or 3 waves of different frequency and the subharmonic waves were also artificially excited. With this strategy, the problem of identification of detuned resonance in the modulated wave system was circumvented because the degree of detuning was established by the excitation, not by the evolution. Although being an important step into modulated waves, the analysis carried out in (PAULA et al., 2013) is still limited relative to the wave packet composed by a continuous spectrum.

\subsubsection{DNS simulations of wave packets}

A Direct Numerical Simulation (DNS) have a high demand for computational resources, hence, they are used mainly as a research tool. A more general comprehensive review of DNS used on research in transition and turbulence can be found in (MOIN; MAHESH, 1998).

Probably Fasel (1976) performed the first DNS simulation for transitional boundary layers, focused on exploring the quality of numerical solutions by comparison with results of linear theory and related experimental observations. Fasel e Konzelmann (1990) quantified the effect of assumptions considered for the derivation of LST, as the non parallel assumption. Due to computational limitations, Fasel (1984) performed first simulations of two-dimensional wave packets. Early three-dimensional DNS simulations of transition in compressible boundary layers, performed along the decade of 1990s, were focused on exploring the potential of the numerical approach, also into developing accurate numerical methods for this kind of problem and improving computational performance, using, for example, parallelization (KARNIADAKIS, 2003).

In the literature, there are three main reference experiments of wave packets on incompressible boundary layer: (GASTER; GRANT, 1975), (COHEN; BREUER; HARITONIDIS, 1991) and (MEDEIROS; GASTER, 1999b). The first two experiments were reproduced using DNS simulations, by (KONZELMANN; FASEL, 1991; SEVERIN, 2012) and (YEO et al., 2010) respectively, leading to numerical investigation. A numerical analysis of the experiment (MEDEIROS; GASTER, 1999b) is not found in literature.

Some observations at Mach 0.5, considering white noise (NORMAND; LESIEUR, 1992), nonlinear growth (WASISTHO; GEURTS; KUERTEN, 1997), transition on an airfoil (LIU et al., 
1997) and secondary instability mechanisms (DENG et al., 2005) suggest that up to Mach 0.5 there are no remarkable differences in the initial nonlinear stages of transition in compressible boundary layer. A remarkable fact of the DNS simulations is the prediction of the oblique transition mechanism, not observed previously in experiments. In the last fifteen years DNS simulations concentrated on initial nonlinear stages and fully developed turbulence (DENG et al., 2005). Recently, investigations have been focused on the determination of the details in the transition process at several Mach numbers, considering changes in the three dimensional structures and the effect of a selected parameter, such as wall temperature and geometry.

Initial works of wave packets on compressible boundary layers are for hypersonic flows (FORGOSTON; TUMIN, 2006), and DNS simulations at Mach 1.3 (PIROZZOLI; BERNARDINI; GRASSO, 2010), Mach 2 (MAYER; WERNZ; FASEL, 2011), Mach 3 (MAYER; TERZI; FASEL, 2011) and several for higher Mach numbers (FRANKO; LELE, 2013; SIVASUBRAMANIAN; FASEL, 2015) were performed later.

Despite a great number of engineering applications falls into high subsonic and transonic regime, the investigations on wave packet evolution concentrate on either incompressible and supersonic boundary layers. Research of wave packet evolution in subsonic boundary layer is still incipient.

\subsection{Objectives of this work}

The present work has two principals objectives: to develop a DNS code focused in problems of transition in subsonic boundary layers on a flat plate and to investigate natural transition in subsonic regime, modeled by wave packets and white noise. Three main problems were considered: numerical simulation of the experiment (MEDEIROS; GASTER, 1999b) in incompressible boundary layer, the influence of compressibility on wave packet evolution at subsonic Mach numbers and finally, a preliminary study of transition induced by white noise in a boundary layer at Mach 0.2 and 0.9 .

The numerical results are analyzed in the context of current nonlinear theories for monochromatic waves, to establish the nonlinear regime that the packet undergo. 


\subsection{Thesis outline}

The document is organized as follows. Chapter 2 describes the governing equations and boundary conditions tested and eventually used. The numerical procedure is also described, including the time and space discretization, filter, grid stretching and parallelization, among other aspects. In Chapter 3, the validation tests performed on the code are presented. This includes tests in the linear and nonlinear regime. Results of subharmonic, fundamental and oblique resonances on incompressible and compressible subsonic boundary layers available in the literature were used for comparison.

In Chapter 4 results for DNS simulation of the experiment (MEDEIROS; GASTER, 1999b) are presented. Detailed comparison on physical and Fourier spaces is shown, a nonlinear modal analysis is performed and several relevant calculations are presented. Chapter 5 is devoted to results for wave packets at Mach 0.7 and 0.9 , including interaction between packets at Mach 0.9. Chapter 6 presents results and preliminary analysis for transition generated by white noise at Mach 0.2 and 0.9 . Finally, Chapter 7 is dedicated to conclusions, final remarks and suggested future works. 


\section{Physical problem and computational \\ setup}

\subsection{Governing equations}

Navier Stokes equations for compressible flow at subsonic Mach numbers were solved numerically in a rectangular integration domain as indicated by dashed lines in the figure 1.2, to calculate the boundary layer over a flat plate. It was considered the non-conservative formulation, in terms of density $\rho$, velocity components $u_{i}$ and internal energy $e$. All variables are non-dimensionalized by the displacement thickness $\delta_{0}^{*}$ at a reference position in streamwise direction, free-stream value of velocity $U_{\infty}^{*}$ and density $\rho_{\infty}^{*}$. The equations can be written as

$$
\begin{aligned}
& \frac{\partial \rho}{\partial t}=-\rho \frac{\partial u_{i}}{\partial x_{i}}-\frac{\partial \rho}{\partial x_{i}} u_{i} \\
& \frac{\partial u_{j}}{\partial t}=-\frac{\partial u_{j}}{\partial x_{i}} u_{i}-\frac{1}{\rho} \frac{\partial p}{\partial x_{j}}+\frac{1}{\rho} \frac{\partial \tau_{i j}}{\partial x_{i}} \\
& \frac{\partial e}{\partial t}=-\frac{\partial e}{\partial x_{i}} u_{i}-\frac{p}{\rho} \frac{\partial u_{i}}{\partial x_{i}}+\frac{1}{\rho} \tau_{i j} \frac{\partial u_{j}}{\partial x_{i}}-\frac{1}{\rho} \frac{\partial q_{i}}{\partial x_{i}}
\end{aligned}
$$

The viscous tensor is defined as

$$
\tau_{i j}=\frac{\mu(T)}{R e}\left[\frac{\partial u_{i}}{\partial x_{j}}+\frac{\partial u_{j}}{\partial x_{i}}-\frac{2}{3} \delta_{i j} \frac{\partial u_{k}}{\partial x_{k}}\right]
$$

and the heat flux term is given as

$$
q_{i}=-\frac{\mu}{(\gamma-1) \operatorname{RePr} M_{\infty}^{2}} \frac{\partial T}{\partial x_{i}} .
$$

The Reynolds, Prandtl and Mach numbers are defined as:

$$
R e=\frac{\rho_{\infty}^{*} U_{\infty}^{*} \delta_{0}^{*}}{\mu_{\infty}^{*}} \quad \operatorname{Pr}=\frac{\mu_{\infty}^{*} c p^{*}}{k^{*}} \quad M_{\infty}=\frac{U_{\infty}^{*}}{c_{\infty}^{*}}=\frac{U_{\infty}^{*}}{\sqrt{\gamma p_{\infty}^{*} / \rho_{\infty}^{*}}}
$$


with $\mu_{\infty}^{*}, c_{p}^{*}$ the references dynamic viscosity and specific heat, and $\gamma$ the heat capacity ratio.

Temperature and internal energy are related by:

$$
e=\frac{T}{\left(\gamma^{2}-\gamma\right) M_{\infty}^{2}}
$$

To complete the set of equations required to solve the Navier-Stokes equations system, the ideal gas hypothesis is considered, to relate the pressure $p$ with the internal energy and density

$$
p=(\gamma-1) \rho e
$$

Finally viscosity is modeled considering the Sutherland's law

$$
\frac{\mu^{*}}{\mu_{\infty}}=\mu(T)=\frac{1+C}{T+C} T^{\frac{3}{2}}
$$

with $C=\frac{110 k}{T_{\infty}^{*}}$, and $T_{\infty}^{*}=300 K$. The relations between dimensional variables denoted with $(*)$ and nondimensional ones are:

$$
\begin{gathered}
\rho=\frac{\rho^{*}}{\rho_{\infty}^{*}}, \quad p=\frac{p^{*}}{p_{\infty}^{*} U_{\infty}^{* 2}}, \quad x_{i}=\frac{x_{i}^{*}}{\delta_{0}^{*}}, \\
T=\frac{T^{*}}{T_{\infty}^{*}}, \quad u_{i}=\frac{u_{i}^{*}}{U_{\infty}^{*}}, \quad t=\frac{t^{*} U_{\infty}^{*}}{\delta_{0}^{*}}, \quad e=\frac{e^{*}}{U_{\infty}^{* 2}} .
\end{gathered}
$$

\section{$2.2 \quad$ Numerical Methods}

A DNS code was developed focusing on problems of transition in boundary layer at subsonic Mach numbers. The code is implemented in Fortran90 and parallelized using MPI (Message Passage Interface). Routines of LAPACK library are used to solve linear equation systems for spatial derivatives calculation

In transition to turbulence problems, the flow shows variations in a wide range of scales, usually between $10^{-6}$ and $10^{-2}$ of the free stream velocity. Then this variations must be calculated, and spectral-like schemes are interesting.

\subsubsection{Discretization}

For spatial discretization a compact finite differences scheme was constructed with spectral like resolution, based on (LELE, 1992). The scheme is $4^{\text {th }}$ order accuracy and is composed by a tridiagonal stencil to reduce computational cost as compared with pentadiagonal implementations. These schemes allow highly accurate discretizations with a lower number of grid points than required in non-compact methods. The time integration is performed with a standard $4^{\text {th }}$ order Runge Kutta method:

$$
y_{n+1}=y_{n}+\frac{1}{6}\left(k_{1}+k_{2}+k_{3}+k_{4}\right)
$$


with:

$$
\begin{aligned}
& k_{1}=\Delta t f\left(t_{n}, y_{n}\right) \\
& k_{2}=\Delta t f\left(t_{n}+\frac{1}{2} \Delta, y_{n}+\frac{1}{2} k_{1}\right) \\
& k_{3}=\Delta t f\left(t_{n}+\frac{1}{2} \Delta, y_{n}+\frac{1}{2} k_{2}\right) \\
& k_{4}=\Delta t f\left(t_{n} \Delta, y_{n}+k_{3}\right)
\end{aligned}
$$

Uniform grid is used in streamwise and spanwise directions, grid is stretched in wall normal direction to increase resolution near the wall and resolve gradients correctly. Details can be found in (BERGAMO, 2013; BERGAMO et al., 2015).

\subsubsection{Compact finite differences}

In an uniform grid the first derivative can be approximated as

$$
\beta f_{i-2}^{\prime}+\alpha f_{i-1}^{\prime}+f_{i}^{\prime}+\alpha f_{i+1}^{\prime}+\beta f_{i+2}^{\prime}=c \frac{f_{i+3}-f_{i-3}}{6 h}+b \frac{f_{i+2}-f_{i-2}}{4 h}+a \frac{f_{i+1}-f_{i-1}}{2 h} .
$$

The coefficients $a, b, c, \alpha$, e $\beta$ are chosen to set the order of stencil with respect to the truncated Taylor series.

$$
\begin{array}{ll}
\text { Second order: } & a+b+c=1+2 \alpha+2 \beta, \\
\text { Fourth order: } & a+2^{2} b+3^{2} c=2 \frac{3 !}{2 !}\left(\alpha 2^{2} \beta\right), \\
\text { Sixth order: } & a+2^{4} b+3^{4} c=2 \frac{5 !}{4 !}\left(\alpha 2^{4} \beta\right), \\
\text { Eigth order: } & a+2^{6} b+3^{6} c=2 \frac{7 !}{6 !}\left(\alpha+2^{6} \beta\right), \\
\text { Tenth order: } & a+2^{8} b+3^{8} c=2 \frac{9 !}{8 !}\left(\alpha+2^{8} \beta\right) .
\end{array}
$$

Relations (20) to (24) can be used to construct up to $10^{\text {th }}$ order accurate stencils. By means of error analysis, it is possible to calculate the modified wave number $w^{\prime}$ as a function of the real wave number $w$ for each finite difference scheme as

$$
w^{\prime}(w)=\frac{a \sin (w)+(b / 2) \sin (2 w)+(c / 3) \sin (3 w)}{1+2 \alpha \cos (w)+2 \beta \cos (2 w)} .
$$

In (LELE, 1992), $4^{\text {th }}$ order pentadiagonal schemes with spectral-like resolution are constructed, using relations (20) and (21) and imposing the condition:

$$
w^{\prime}\left(w_{1}\right)=w_{1}, \quad w^{\prime}\left(w_{2}\right)=w_{2}, \quad w^{\prime}\left(w_{3}\right)=w_{3}
$$

To reduce the computational cost associated with a pentadiagonal system, on the right hand side of the equation 19 two approximations were considered to construct tridiagonal 
schemes, with stencil of 5 and 7 points $(\beta=0)$, both $4^{\text {th }}$ order accurate. Using the relations (20) and (21), the 5 point stencil was obtained by imposing

$$
c=0, \quad w^{\prime}\left(w_{1}\right)=w_{1}
$$

the 7 point stencil results from

$$
w^{\prime}\left(w_{1}\right)=w_{1}, \quad w^{\prime}\left(w_{2}\right)=w_{2}
$$

Several schemes were tested by solving the advection equation to compare the modified wave number and phase speed $c_{p}=w^{\prime} / w$, as shown in figures 2.1 and 2.2.
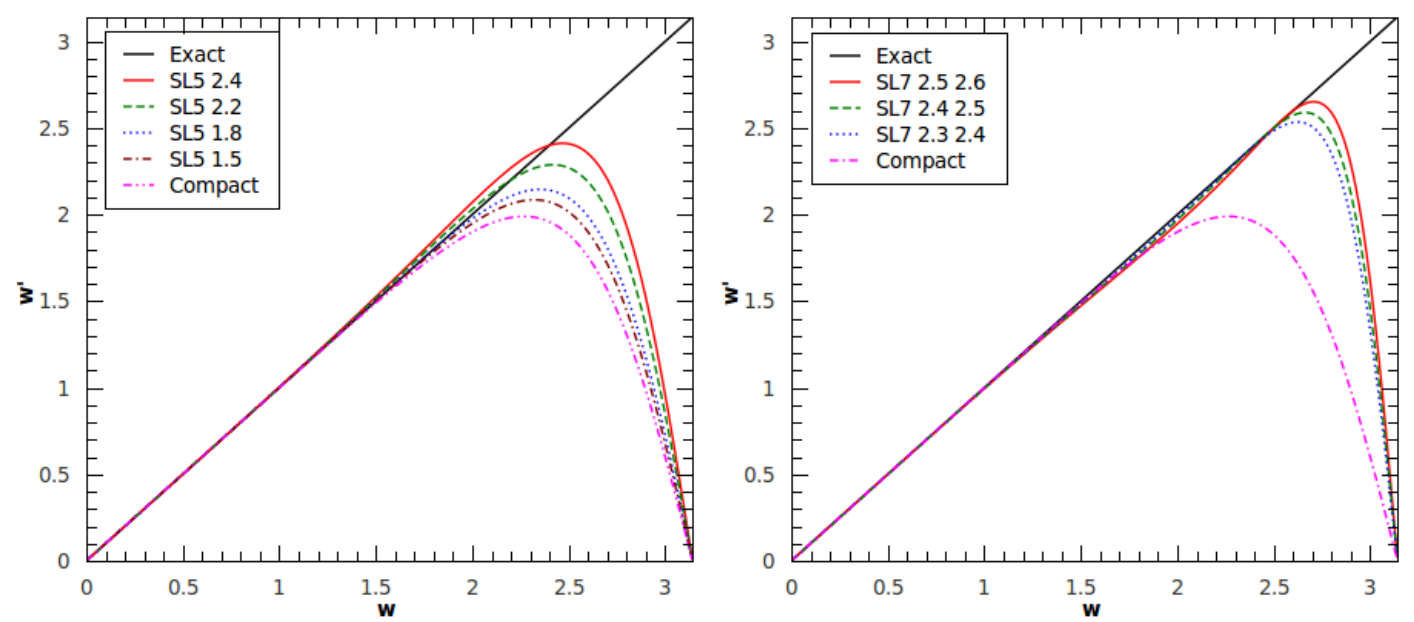

Figure 2.1: Modified wave number for several schemes. (a) 5 points stencil for $w_{1}=1.5$; $w_{1}=1.8 ; w_{1}=2.2$ and $w_{1}=2.4$. (b) 7 points stencil to $w_{1}=2.3, w_{2}=2.4 ; w_{1}=$ $2.4, w_{2}=2.5 ; w_{1}=2.5, w_{2}=2.6$. Comparison with tridiagonal sixth order scheme. (Reproduced from (BERGAMO, 2013)).
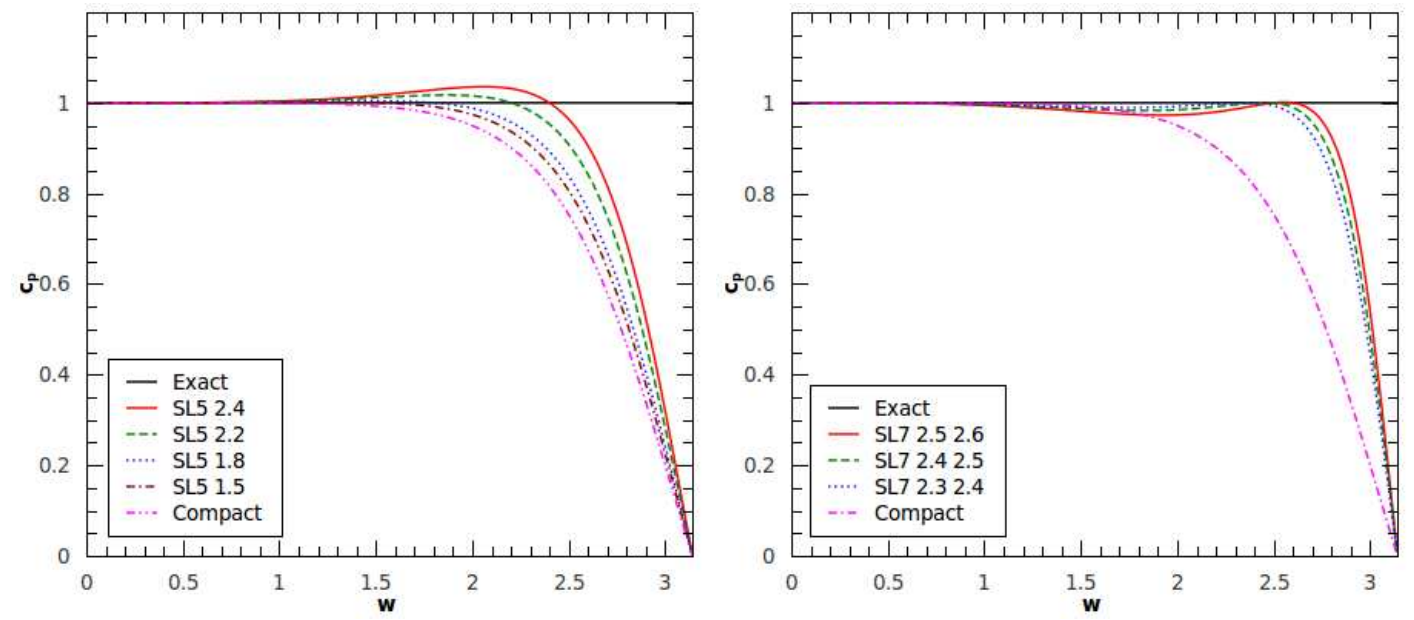

Figure 2.2: Phase velocity for several schemes. (a) 5 points stencil for $w_{1}=1.5 ; w_{1}=1.8$; $w_{1}=2.2$ and $w_{1}=2.4$. (b) 7 points stencil to $w_{1}=2.3, w_{2}=2.4 ; w_{1}=2.4, w_{2}=2.5$; $w_{1}=2.5, w_{2}=2.6$. Comparison with tridiagonal sixth order scheme. (Reproduced from (BERGAMO, 2013)). 
From the modified wave number curves and phase velocity for 5 -point stencil, $\omega^{\prime}=1.8$ was chosen because it offers the best approximation. By substitution into restrictions (20, $21,25)$, the resulting stencil is:

$$
\alpha=0.364957272268410 \beta=0, a=1.57663818151227 b=0.153276363024547 c=0
$$

This stencil was employed in all simulations performed in the present work.

\subsubsection{Numerical Stability}

For the advection equation this integration scheme is stable up to CFL (Courant-FriedrichsLewy) number around 1.3 (LELE, 1992)[pag. 32]. For the Navier-Stokes equations the time step was modified, also this value of CFL was achieved to obtain a numerically stable solution. The convection and diffusion stability criterion are given by:

$$
\Delta t \leq \frac{C F L}{\left[\frac{\frac{1}{M_{\infty}}+u_{\max }}{\Delta x}+\frac{\frac{1}{M_{\infty}}+v_{\max }}{\Delta y}+\frac{\frac{1}{M_{\infty}}+w_{\max }}{\Delta z}\right]}
$$

\subsubsection{Filter}

Spurious oscillations are generated by numerical boundary conditions, grid stretching, among other factors. This nonphysical oscillations may be amplified affecting the numerical solution. To control spurious oscillations a $10^{\text {th }}$ order low pass filter (VISBAL; GAITONDE, 2002) was applied in each time iteration at the inner points of the domain. In the spanwise direction, a periodic filter is used to be compatible with the periodic boundary condition. The filtering level is controlled with the numerical parameter $\alpha$, which varies from $-0.5 \leq \alpha_{f} \leq 0.5$, low values of $\alpha_{f}$ correspond to higher filtering. In all simulations presented here $\alpha_{f}=0.49$ was employed in all directions, which means almost no filter. For an arbitrary discrete variable $\phi$ the filter is defined by:

$$
\alpha_{f} \bar{\phi}_{i-1}+\bar{\phi}_{i} \alpha_{f}+\bar{\phi}_{i+1}=\sum_{n=0}^{N} \frac{a_{n}}{2} \phi_{i+n}+\phi_{i-n}
$$

The coefficients for the $10^{\text {th }}$ order filter are:

$$
\begin{array}{ccc}
a 0=\frac{193+126 \alpha_{f}}{256} & a 1=\frac{105+302 \alpha_{f}}{256} & a 2=15 \frac{-1+2 \alpha_{f}}{64} \\
a 3=\frac{45-2 \alpha_{f}}{512} & a 4=5 \frac{-1+2 \alpha_{f}}{256} & a 5=\frac{1-2 \alpha_{f}}{512}
\end{array}
$$




\subsubsection{Grid stretching}

The domain was discretized with an uniform grid in $\mathrm{x}$ and $\mathrm{z}$ directions, in the wall normal direction a stretched grid was employed. The number of grid points considered in each direction were $\mathrm{nx}, \mathrm{nz}$ and ny respectively. For the simulations performed in this work, two grids in $y$-direction were considered, one defined by $0 \leq y \leq 20, n y=51$ and a more refined grid with an wider wall normal domain defined by, $0 \leq y \leq=60, n y=151$, both, are shown in figure 2.3 .

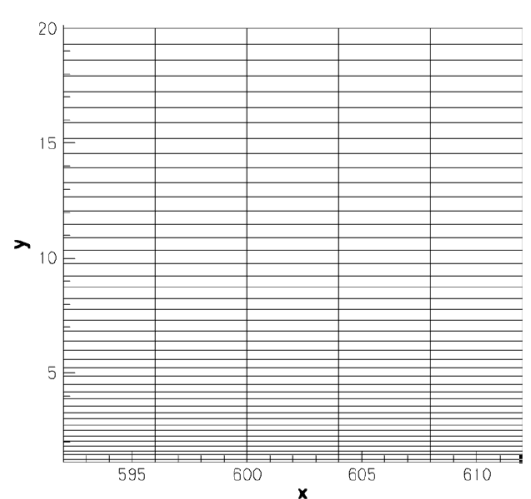

(a)

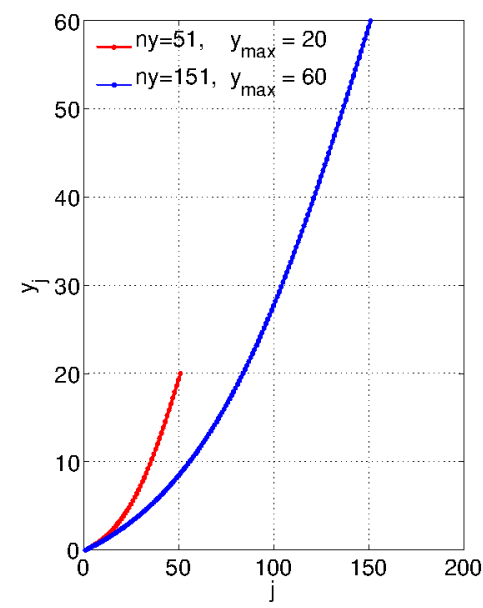

(b)

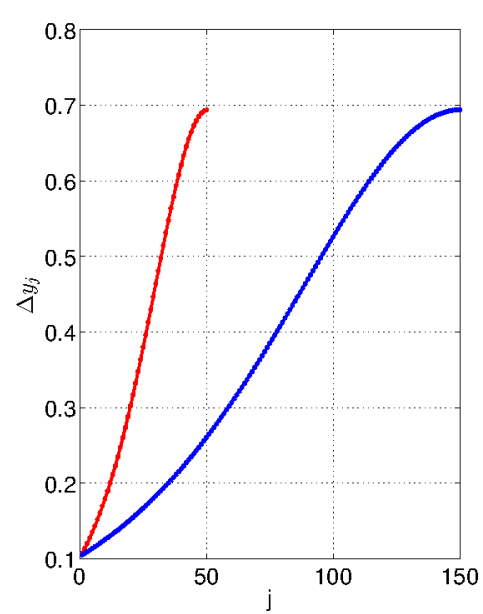

(c)

Figure 2.3: (a) Grid stretching in the wall normal direction, (b) stretching function and (c) cell size variation.

\subsubsection{Boundary Conditions}

Initially characteristic boundary conditions were considered to be used in the boundary layer simulations. As a code test, reflecting and non-reflecting outflow boundary conditions proposed by (POINSOT; LELE, 1992) were applied to the Poiseuille flow. This approach calculates the numerical boundary conditions based on considerations and approximations of Navier-Stokes equations, to control wave reflections. Results shown in figures 2.4 and 2.5 reveal a good qualitative agreement, including details in the contour levels of the flow variables. This kind of boundary conditions was not used in the boundary layer, because in their formulation they have parameters strongly dependent of the problem that must be determined empirically. Also, a buffer zone is required (WASISTHO; GEURTS; KUERTEN, 1997), which does not justify the complexity and the increase in the computational time. 
(a)

Reflecting Outflow
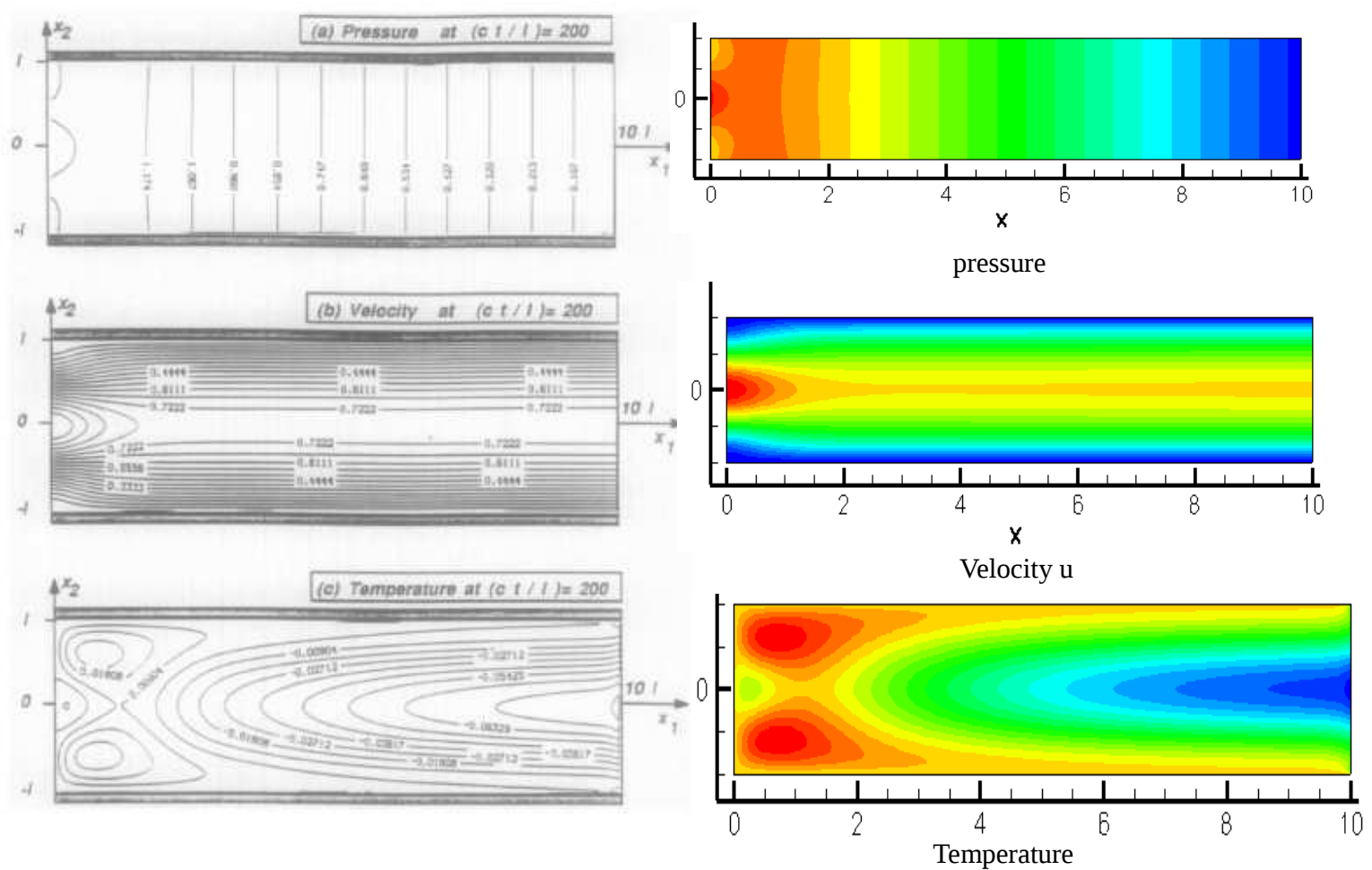

iterations: 100000

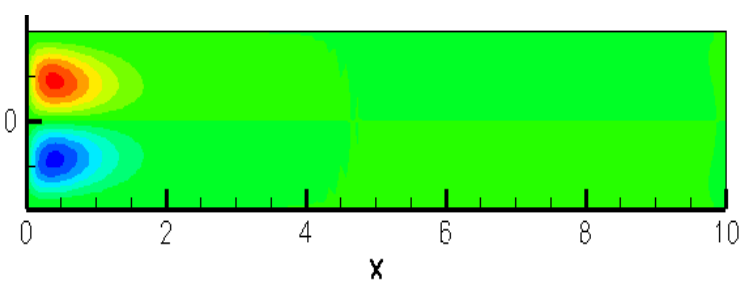

Velocity v

Figure 2.4: Reflecting outflow boundary conditions applied to the Poiseuille flow in a two-dimensional domain. (a) Reference case (Reproduced from (POINSOT; LELE, 1992)) and (b) DNS simulation are in a very good agreement. 
(a)

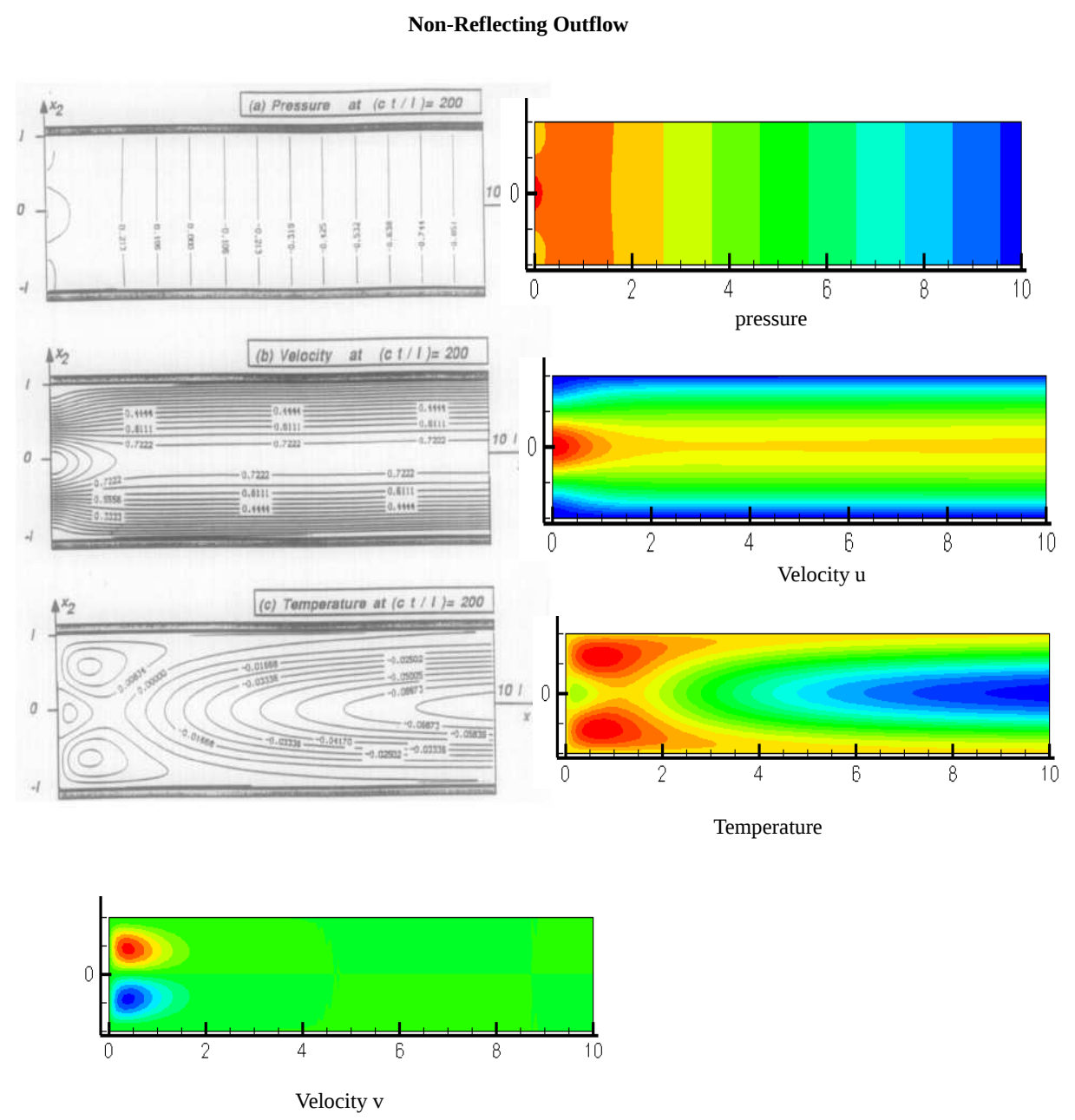

Figure 2.5: Non-reflecting outflow boundary conditions applied to the Poiseuille flow in a two-dimensional domain. (a) Reference case (Reproduced from (POINSOT; LELE, 1992)) and (b) DNS simulation are in a very good agreement.

In the transitional boudary layer simulations, the base flow is a boundary layer over a flat plate, which is obtained with a two-dimensional DNS simulation of the long semiinfinite plate. At the inflow, uniform flow condition is imposed by fixing velocity, with $u=1$ and zero for the others velocity components whereas the pressure is calculated with Neumann condition. At the outflow the pressure is fixed, and the other flow variables are calculated using null second derivative. Also a buffer zone is included at the outflow to avoid reflections into the integration domain. On the wall, a no-slip condition is applied to 
the velocity components meanwhile isothermal wall is used for the temperature, density is calculated with a compatibility condition for the pressure. Finally at the upper boundary, Neumann condition is applied to the velocity components and density, the pressure is fixed. In three dimensional simulations, periodic boundary condition is used in the spanwise direction.

To obtain the steady state base flow, the simulation is run until variations of the integrated variables are of order $10^{-12}$ between consecutive time steps. To better represent free flow condition at the leading edge of the plate, a domain extension upstream of the plate is included with free-slip condition at the wall, as sketched in figure 2.6. With this set up, some undesired and nonphysical pressure gradient which results in small velocity profiles deformations are avoided.

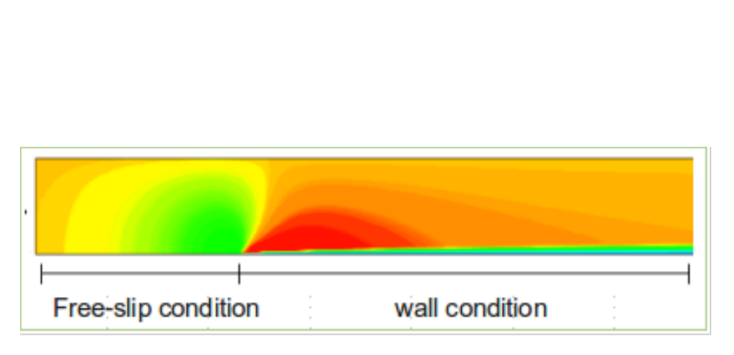

(a)

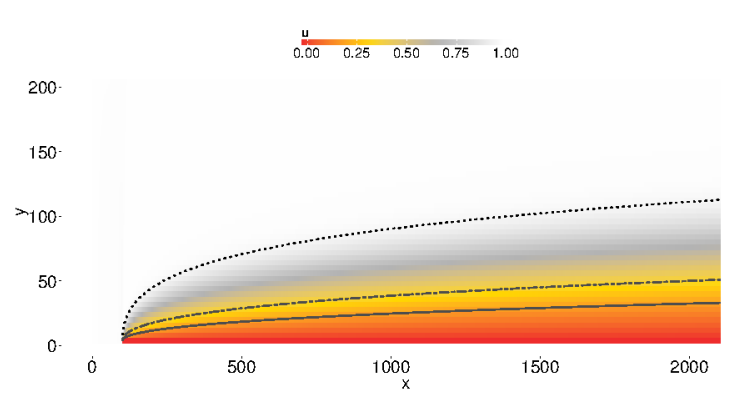

(b)

Figure 2.6: Steady state boundary layer profile generation. (a) Upstream of the leading edge of the flat plate a free-slip wall is included to avoid nonphysical gradients associated with the plate leading edge. (b) Boundary layer profile. (Figure is stretched in y direction to facilitate visualization) $0.6 \delta^{*}(-), 1.1 \delta^{*}(-.-)$ and $\delta_{99}(\ldots)$.

\subsubsection{D Moving Frame}

To reduce the computational domain and hence the number of grid points, a three dimensional moving frame was implemented. During the simulation the domain was increased downstream and in the spanwise direction, and reduced upstream, to perform calculation only in the region occupied by the wave packet. The domain was resized sometimes along the simulation. Figure 2.7 shows three different computational domains superposed to evidence the differences. Several tests performed by comparison of results obtained for several frames sizes show that there are no significant differences in the packet region or at the boundaries, in physical and Fourier spaces. 


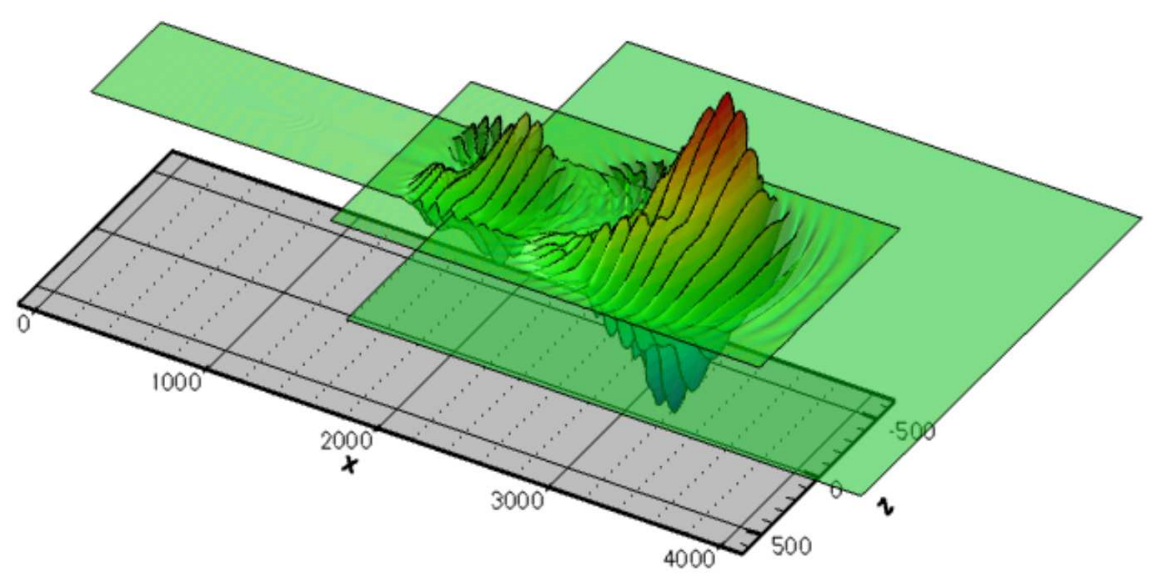

Figure 2.7: Moving frame 3D.

\subsection{Parallelization}

The code was parallelized employing domain decomposition technique by using the library (2DECOMP\&FFT, ). This implementation allows to divide the computational domain into $N \times 1$ or $N \times M$ blocks, sometimes named as slab and pencil decomposition respectively, as sketched in figure 2.8. In this kind of decomposition each processor stores a sub-domain, that contains all axis points in one direction, so that all derivatives in this direction can be calculated in parallel with a compact finite difference scheme.

To calculate the derivatives in the other directions, the orientation of the decomposition is changed. The orientation change is performed by transposition operations which involves communication between process, using the mpi_alltoall routine, which is the principal source of overhead in this kind of parallelization. Depending on the employed computer architecture, there is an optimal decomposition, that minimizes the communication time. The parallelization used in this work does not introduce any additional error at the sub-domain boundaries, as occurs for other parallelization approaches in combination with compact schemes (SENGUPTA; DIPANKAR; RAO, 2007), however the overhead in communication operations increase with the number of processors. 


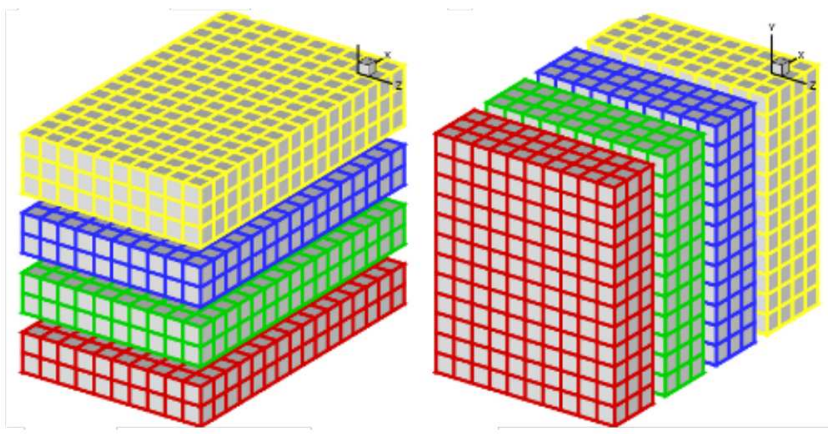

(a)
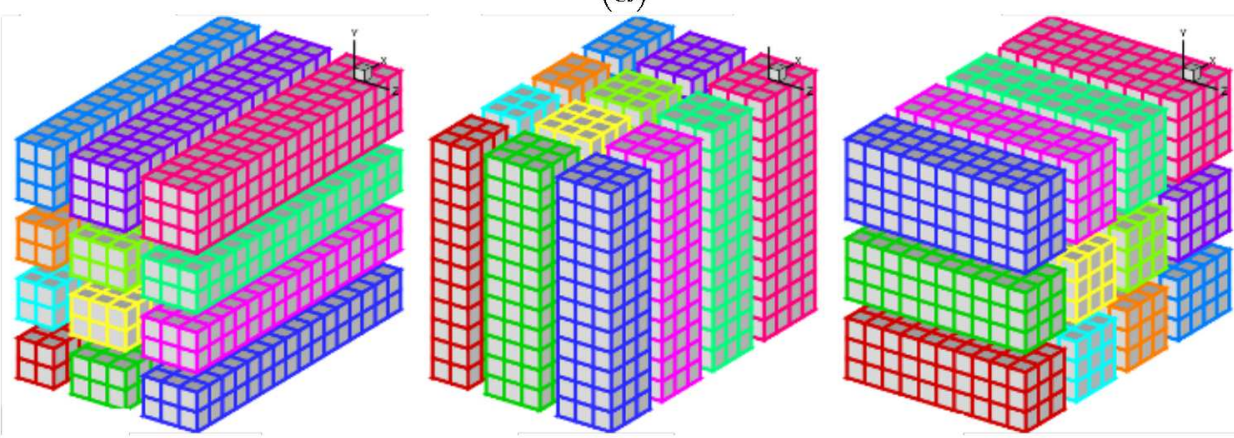

(b)

Figure 2.8: (a) Slab (b) pencil decomposition. (Reproduced from (2DECOMP\&FFT, ).)

\subsubsection{Performance estimation}

There are two kinds of tools to determine program performance: profiling and tracing. Profiling tools allows to collect global data from statistical sampling of events during execution of the program, gprof is an open-source tool of this kind. Tracing tools, as for example Vampir-trace, collect detailed data during execution. This kind of tools are more precise, but larger data files than in profiling tools are generated and can produce substantial computing overhead. To determine the computational performance of the current DNS code, gprof was used for the sequential execution and Vampire-Trace for parallel execution.

\section{Sequential execution}

Using gprof, the code structure can be decomposed into blocks, as shown in figures 2.9 and 2.10. Each box corresponds to a function of the code. Inside the boxes there are 3 numbers indicating the percentage of total computational time employed by a particular function. The second number, between parentheses, indicates the amount of work done by the function and the third number is the number of calls of the function. Numbers outside the boxes are the percentage of work passed to the next function and number of calls. From this diagram it can be concluded that $50 \%$ of the computational time is employed into spatial derivative calculation and the most expensive function is dgtsv of 
(LAPACK, ) library, which was used to solve the tridiagonal system of the finite difference method. Time per/iteration per/grid point is of the order of $5 \mu s$.

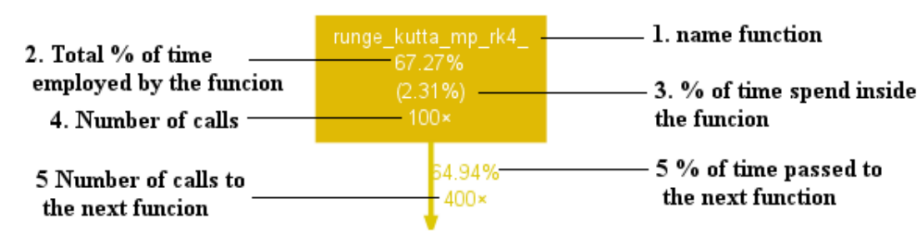

Figure 2.9: Execution diagram and computational cost obtained with gprof.

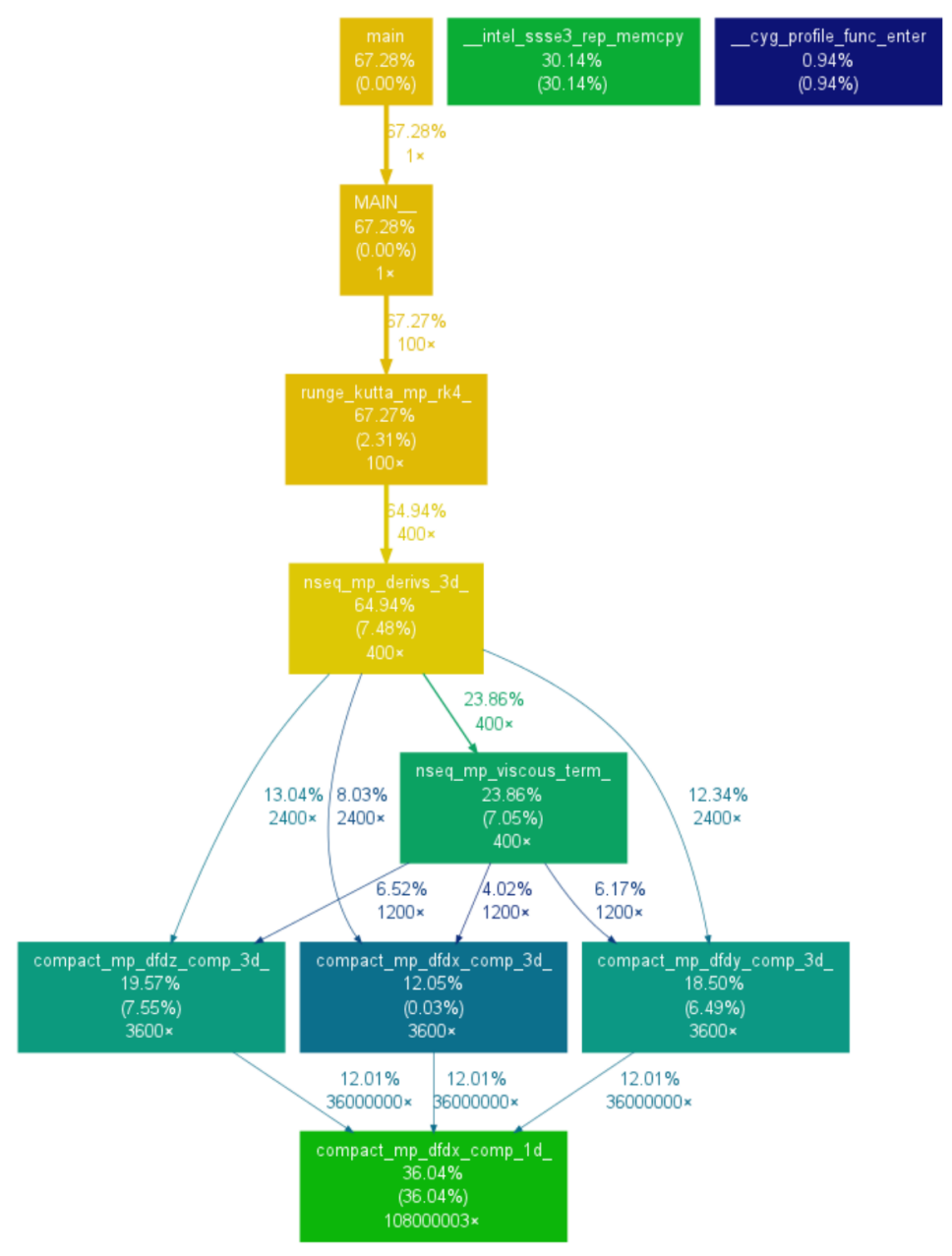

Figure 2.10: Information of the computational cost obtained with gprof. 


\section{Parallel execution}

By using vampir, detailed diagrams of the communication operations between processors can be obtained (figure 2.12). They allow to find errors and bottlenecks to plan optimizations. However, this kind of analysis requires detailed work and only preliminary tests were performed. To evaluate performance of a parallelized program there are several metrics, for the DNS code, the most relevant metric is the speedup. The speedup is a measure of efficiency on the time reduction by increasing number of processors. Ideally, if $\mathrm{n}$ processors are used, the computational time is reduced by a factor of $\frac{1}{n}$. However, due to the communication operations between processors required for the parallelization, an overhead in the computational time is generated, which increases with the processors number. Figure 2.11 shows the results for tests using up 130 processors, carried out for the DNS code, showing a reasonable performance. However, scalability has a strong dependence on the computer architecture and physical communication channels between processors. To perform the simulations of the present work, less than 40 processors were used, for which the performance is better.

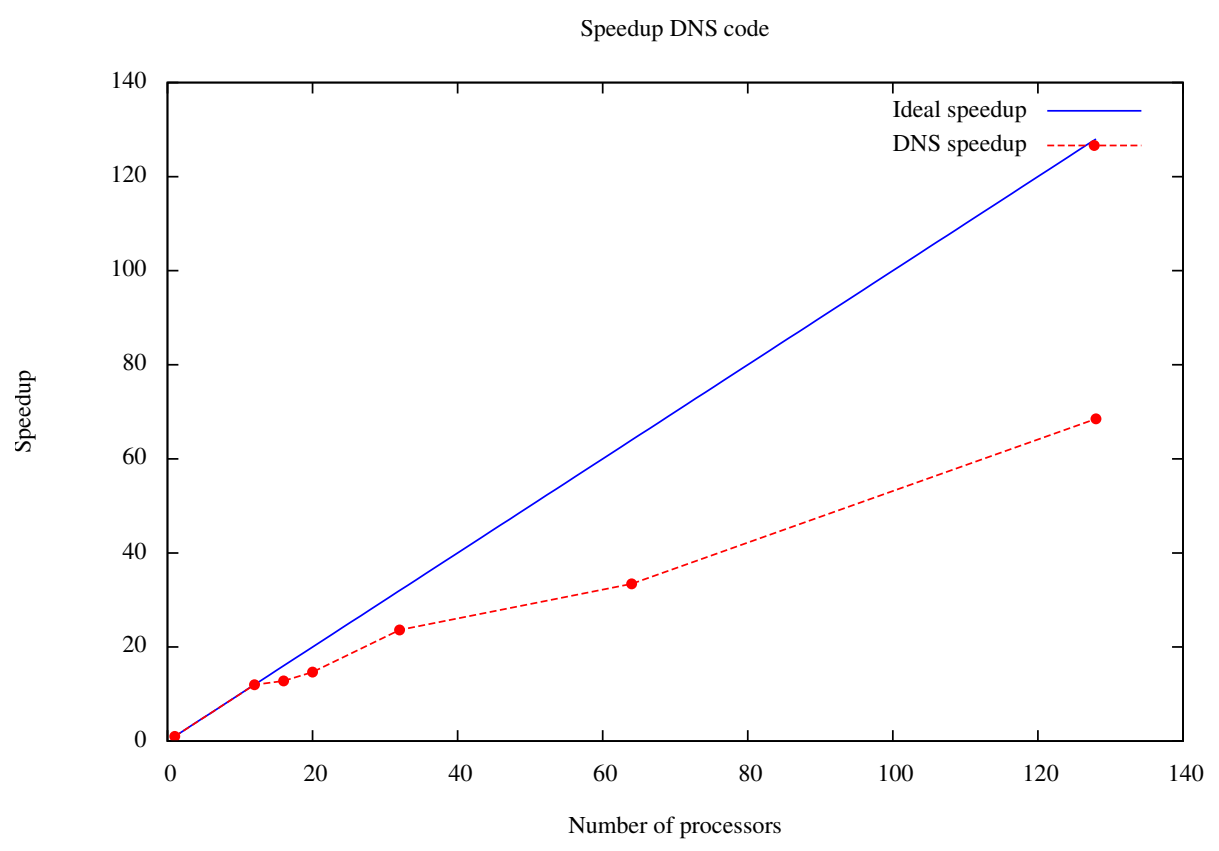

Figure 2.11: Speedup of the DNS code. 


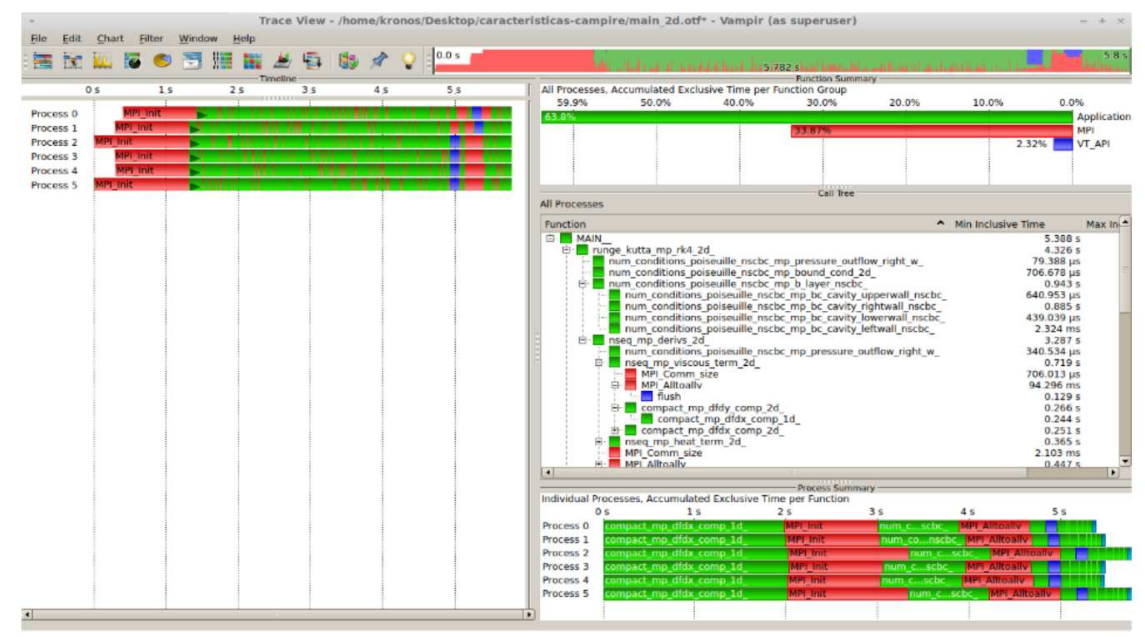

Figure 2.12: Diagrams of profiling obtained with vampir, showing detailed information of the communication operations required for parallelization. 


\section{Code validation}

The developed DNS code has been successfully applied to study several hydrodynamic instability problems in cavity flow (BERGAMO et al., 2015) and boundary layer (MARTINEZ; GENNARO; MEDEIROS, 2015; MARTINEZ; MEDEIROS, 2016). In this chapter, code validation tests are presented at several regimes of transition for subsonic boundary layers. Initially, comparisons of boundary layer profiles with theoretical results are shown. Then, the subsequent sections are devoted to comparisons between DNS simulations and results of the literature for primary and secondary instabilities.

\subsection{Base flow generation}

The base flow considered is a subsonic boundary layer over a flat plate. It is calculated with a two-dimensional DNS simulation starting from an uniform flow until a stationary regime is reached with variations on the integrated variables of the order of $10^{-12}$.

\subsubsection{Boundary layer profiles}

To compare the boundary layer profiles generated with DNS simulations, the Stewartson equations, for compressible boundary layer (see (ANDERSON, 1989)), were solved.. The differential equation system to be solved in terms of the similarity variables $F$ and $T$ is:

$$
\begin{gathered}
\frac{d}{d \eta}\left(\frac{\chi}{P_{r}} \frac{d T}{d \eta}\right)+F \frac{d T}{d \eta}+(\gamma-1) M^{2} \chi\left(\frac{d^{2} F}{d \eta^{2}}\right)^{2}=0 \\
\frac{d}{d \eta}\left(\chi \frac{d^{2} F}{d \eta^{2}}\right)+F \frac{d^{2} F}{d \eta^{2}}=0
\end{gathered}
$$

with $\chi=\rho^{\prime} \mu^{\prime}$. The boundary conditions are:

$$
\left.T\right|_{\eta=0}=T_{w},\left.\quad \frac{d F}{d \eta}\right|_{\eta=0}=0, \quad,\left.F\right|_{\eta=0}=0
$$


and

$$
\left.T\right|_{\eta \rightarrow \infty} \rightarrow 1,\left.\quad \frac{d F}{d \eta}\right|_{\eta \rightarrow \infty} \rightarrow 1
$$

Flow variables are recovered using the transformation:

$$
\begin{gathered}
\eta=\sqrt{\frac{R e}{2 x}} \int_{0}^{y} \rho(x, \tilde{y}) d \tilde{y} \\
u=\frac{d F}{d \eta}, \quad v=T(\eta u-F) \sqrt{\frac{1}{2 x R e}}
\end{gathered}
$$

and

$$
T=T(\eta), \quad \rho=1 / T, \quad e=\frac{1}{\gamma M^{2}(\gamma-1)}+\frac{1}{2}\left(u^{2}+v^{2}\right) \rho
$$

To include the Sutherland's law, $\chi$ is given by:

$$
\chi=\frac{1+C}{T+C} \sqrt{T} \quad \frac{d \chi}{d T}=\chi\left\{\frac{1}{2 T}-\frac{1}{T+C}\right\}
$$

Then, the system of equations 33 and 34 becomes:

$$
\begin{gathered}
\frac{d^{3} F}{d \eta^{3}}=-\frac{1}{\chi} \frac{d^{2} F}{d \eta^{2}}\left\{\frac{d \chi}{d T} \frac{d T}{d \eta}+F\right\} \\
\frac{d^{2} T}{d \eta^{2}}=-\frac{1}{\chi}\left\{\frac{d \chi}{d T}\left\{\frac{d T}{d \eta}\right\}^{2}+P_{r} F \frac{d T}{d \eta}+P_{r}(\gamma-1) M^{2} \chi \frac{d^{2} F}{d \eta^{2}}\right\}=0
\end{gathered}
$$

The Blasius solution (WHITE, 1974) is the theoretical result for incompressible boundary layer. In figure 3.1 the velocity profiles are compared in terms of the similarity variable $\eta=y \sqrt{\frac{R e}{2 x}}$. Comparison of asymptotic values for $\mathrm{v}$ are in a very good agreement.

Also the figure shows the asymptotic value of the wall-normal velocity component, $v$, calculated with the three solutions.

The shape factor $H=\delta^{*} / \theta$, calculated with the Blasius and Stewartson solutions, and DNS simulation are respectively: $H_{\text {Blasius }}=2.591566, H_{S t w}=2.597096, H_{D N S}=$ 2.594004. Flow parameters used in DNS simulations were Mach 0.2 and $\operatorname{Re}=835, \operatorname{Pr}=$ 0.71. All the comparisons performed are in a very good agreement with the theoretical results. 

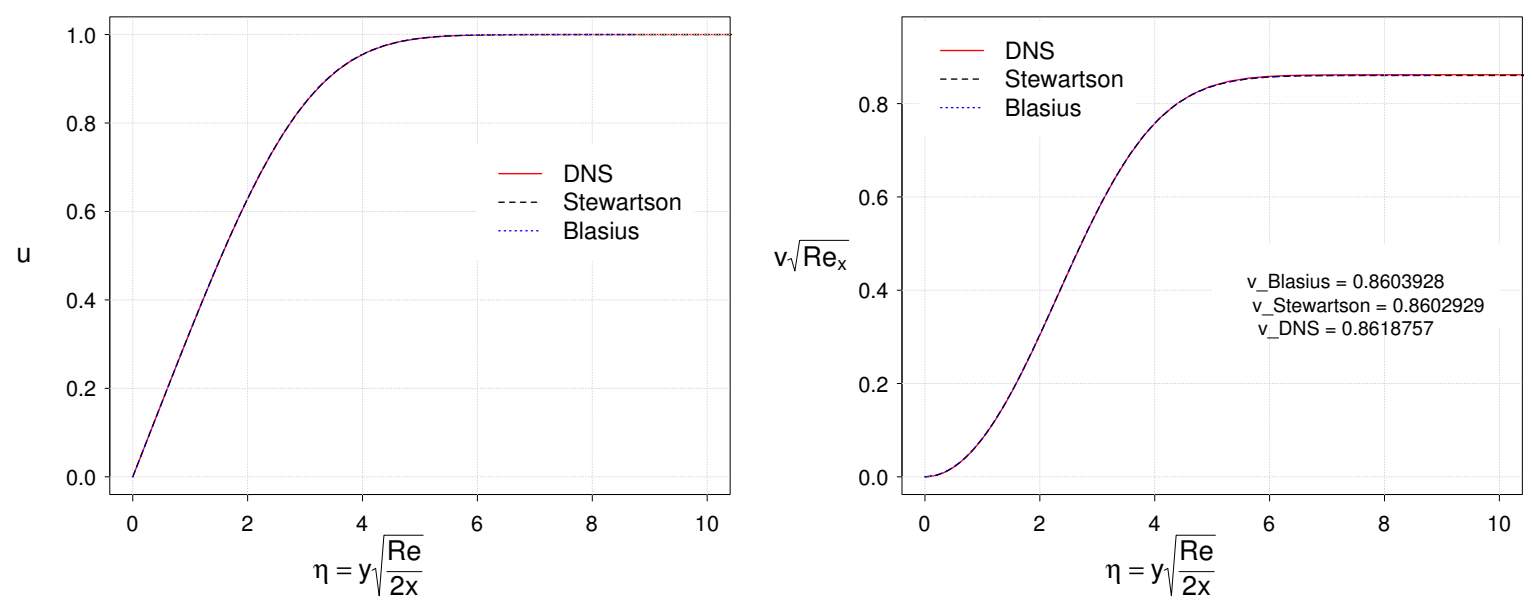

Figure 3.1: Comparison of Blasius profiles for velocity components, $u$, $v$, with Stewartson solution and DNS results. Comparison of asymptotic values for $\mathrm{v}$ are in a very good agreement.

Figure 3.2 shows the thermal boundary layer for adiabatic wall condition, calculated with Stewartson solution and computed with DNS. The value of the adiabatic wall temperature $T_{a d}$, (WHITE, 1974, pag. 511), is also compared by using the theoretical relation

$$
T_{a d}=T_{\infty}\left(1+r \frac{\gamma-1}{2} M^{2}\right)
$$

The recovery coefficient $\mathrm{r}$, for the laminar boundary layers is $r \approx \sqrt{\operatorname{Pr}}$.

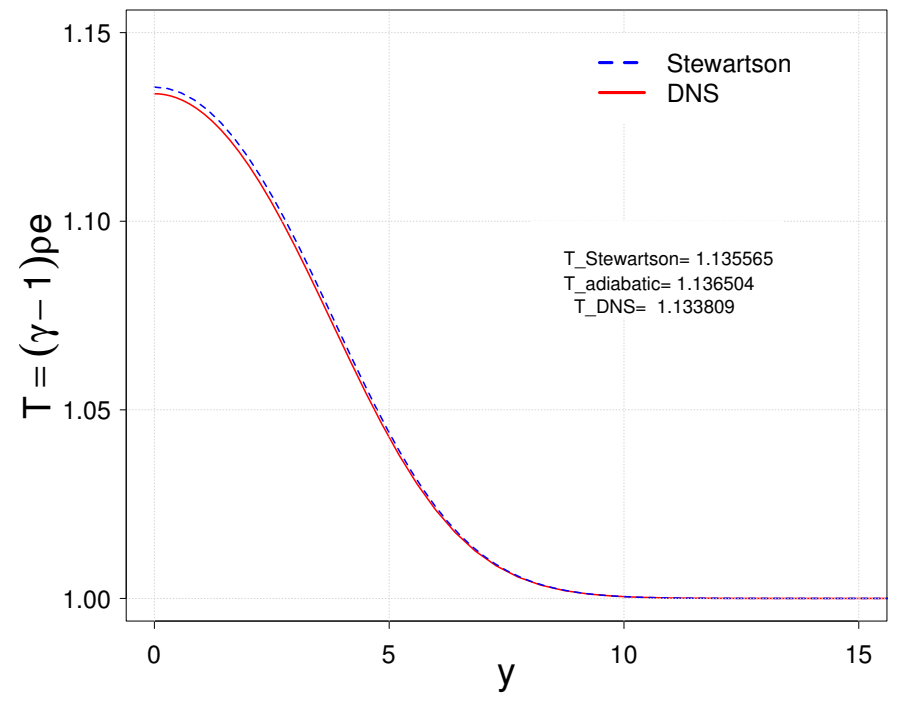

Figure 3.2: Temperature profile for adiabatic wall and comparison of $T_{a d}$ values calculated from DNS, Stewartson solution and theoretical value (equation 43) at Mach 0.9.

All DNS results are in very good agreement with the theoretical results. 


\subsection{Hydrodynamic instability tests}

(KONZELMANN; FASEL, 1991) presented results of a DNS simulation for a TS-wave, in a attempt to investigate the effect of not considered factors in LST, such as non-parallelism. Figure 3.3 shows a comparison of the amplification curves, for a TS-wave with $F=120$. The perturbation is introduced into the flow by using a localized periodic blowing and suction disturbance, applied at the wall normal component of velocity as :

$$
v_{\text {wall }}=A_{1,0} \cos \left[\alpha_{x}\left(x-x_{0}\right)\right] \cos (\omega t)
$$

(a)
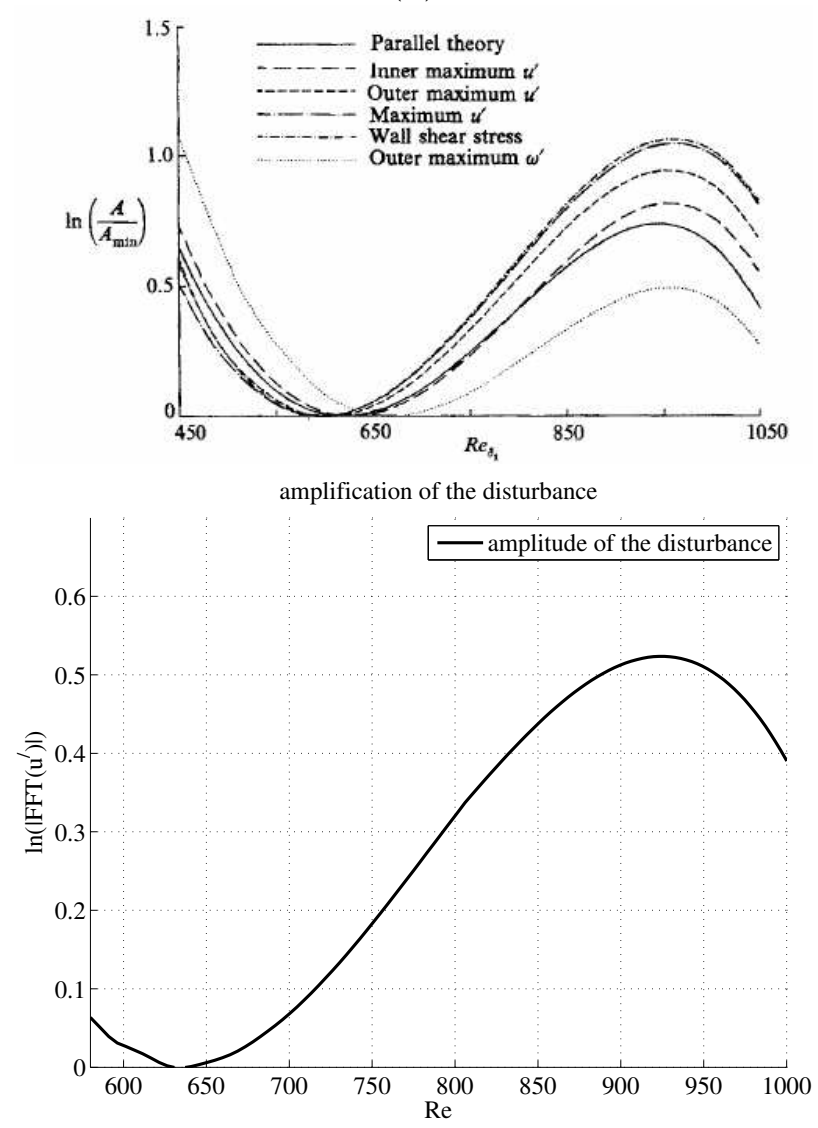

(b)

Figure 3.3: Tollmien-Schlichting amplification curves for incompressible boundary layer. (a) Reproduced from (KONZELMANN; FASEL, 1991, pag. 331) and (b) DNS simulation.

From DNS simulation, the amplification curve employing the inner maximum criterion was calculated, (figure 3.3(b)). Comparison of the curve and the location of the first and second branch with the reference result (figure 3.3(a)) are in a good agreement. 
The results for fundamental resonance on incompressible boundary layer are limited in the literature. For comparison with the current DNS code, (RIST; FASEL, 1995) was used as reference. The perturbation function is defined as

$$
v_{\text {wall }}=A_{1,0} \cos \left[\alpha\left(x-x_{0}\right)\right] \cos (\omega t)+A_{1,1}\left[\cos \left(\alpha\left(x-x_{0}\right) \pm \beta z\right)\right] \cos (\omega t)
$$

Amplification curves obtained from DNS simulation are compared in figure 3.4(a) with the reference case. For the fundamental $(1,0)$, oblique $(1,1)$ and harmonic $(1,2)$ mode, the curves are in very good agreement. The vortical structures generated (figure 3.4(b)) are aligned as expected. In the spectrum shown in figure 3.4(c), the generation and amplification of harmonics, in frequency $(\mathrm{n})$ and spanwise wave numbers $(\mathrm{k})$ can be seen.

(a)

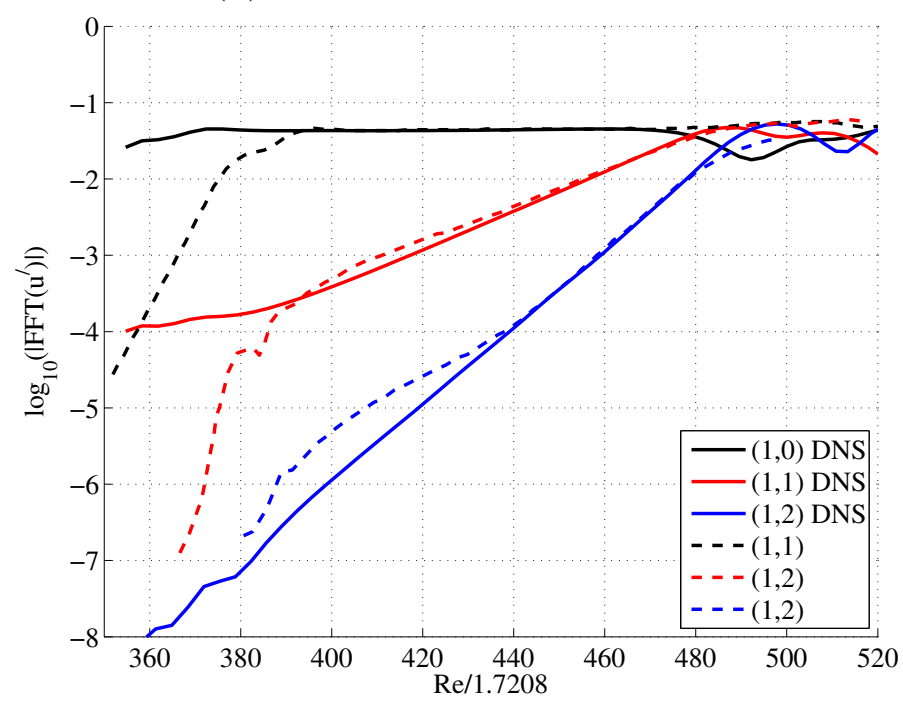

(b)
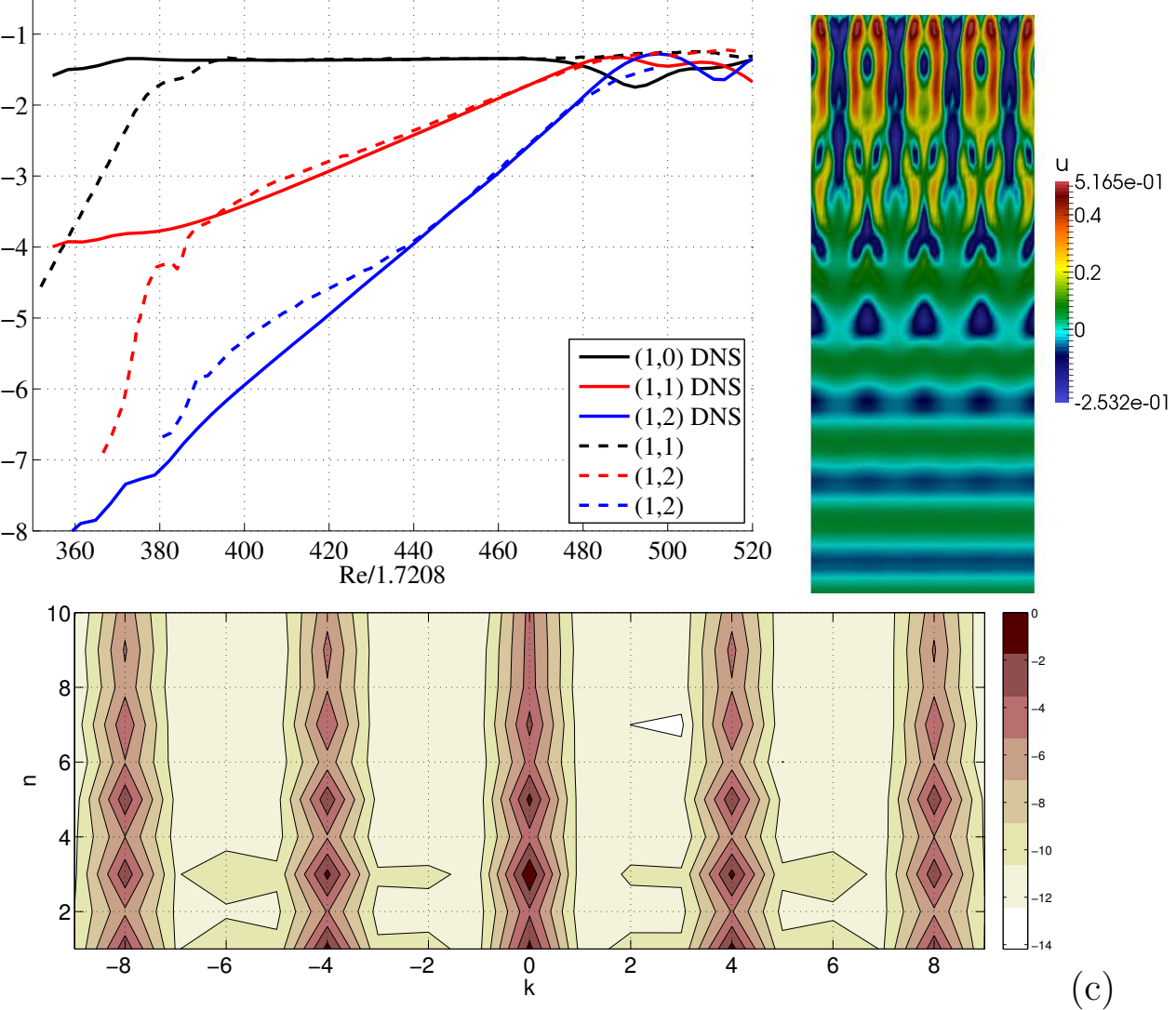

(c)

Figure 3.4: DNS simulation of Fundamental resonance. (a) Comparison of amplification curves with (RIST; FASEL, 1995), (b) Vortical structures generated by the K-type resonance are aligned. (c) Spectrum at $R e / 1.7208=460$ at $y=0.6 \delta^{*}(x)$.

For the subharmonic resonance, (KACHANOV; LEVCHENKO, 1984) was here considered as reference case. The disturbance used to induce H-type resonance was:

$$
v_{\text {wall }}=A_{1,0} \cos \left(\alpha_{x}\left(x-x_{0}\right)\right) \cos (\omega t)+A_{1 / 2,1}\left[\cos \left(\alpha\left(x-x_{0}\right) \pm \beta z\right) \cos ((\omega / 2) t)\right.
$$


Figure 3.5(a) compares the amplification curves for fundamental and subharmonic modes showing good agreement. The resulting vortex array (figure 3.5(b)) is organized in a staggered pattern, as expected for this mechanism. Finally in the spectrum (figure 3.5(c)), the generation and amplification of harmonics in frequency and spanwise wave number can be observed.

To test the code for nonlinear regime in compressible boundary layer, a subharmonic case at Mach 0.8 (MASAD; NAYFEH, 1991) was simulated. Comparison of the amplification curves (figure 3.6(a)) also shows good agreement.

Finally, the case reported by (JOSLIN; STREETT; CHANG, 1993), for oblique resonance was simulated, using as perturbation a pair of oblique waves:

$$
v_{\text {wall }}=A_{1,1}\left[\cos \left(\alpha\left(x-x_{0}\right) \pm \beta z\right)\right] \cos (\omega t)
$$

The comparison of amplification curves for several modes, shown in figure 3.7(a) evidences good agreement between DNS simulations with the reference results. Longitudinal vortical streaks are generated (figure 3.7(b) ) and in the spectra, amplification of the modes $(0,2)$ and $(2,2)$, typical of this kind of nonlinear activity, is observed.

(a)

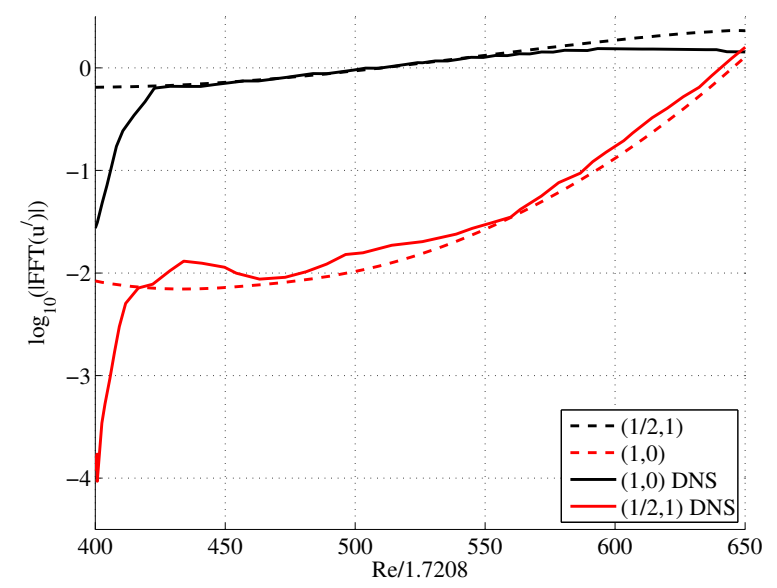

(b)
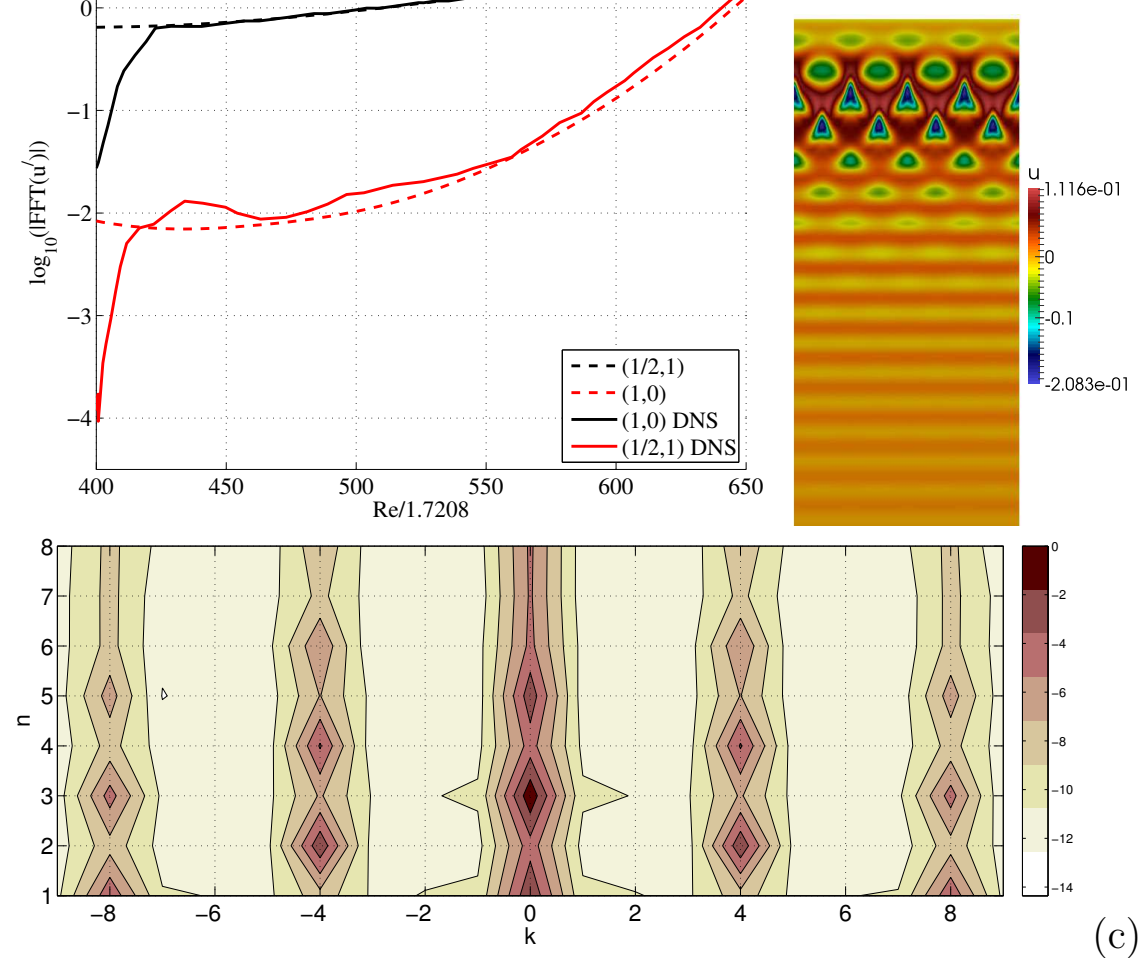

(c)

Figure 3.5: Comparison of Subharmonic resonance from (KACHANOV; LEVCHENKO, 1984) and DNS simulation. (a) Amplification curves, (b) staggered vortex pattern and (c) spectral content. 


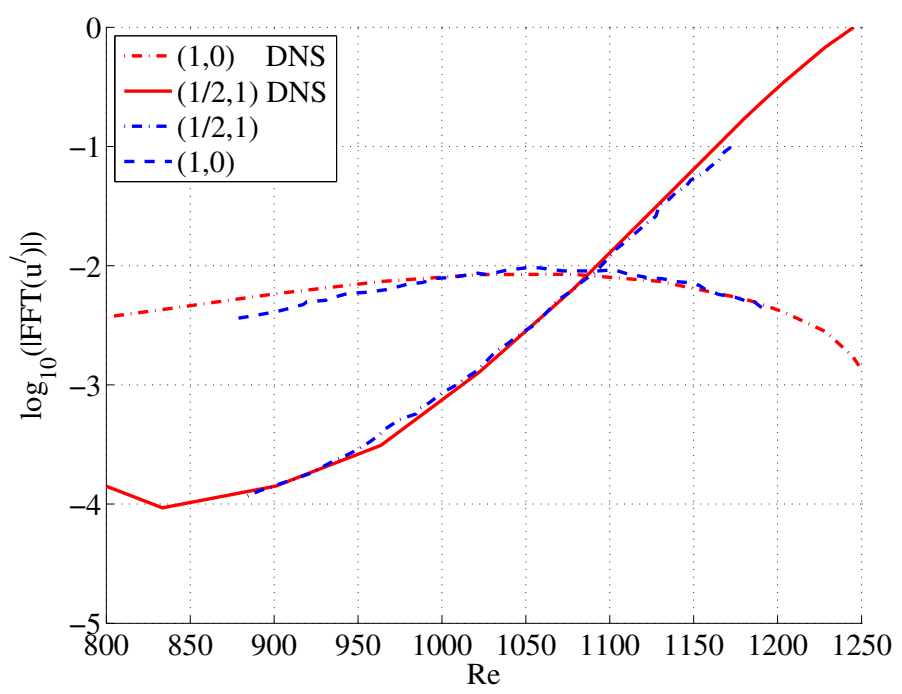

Figure 3.6: Comparison of fundamental and subharmonic waves reported in (MASAD; NAYFEH, 1991) and calculated with DNS.

(a)

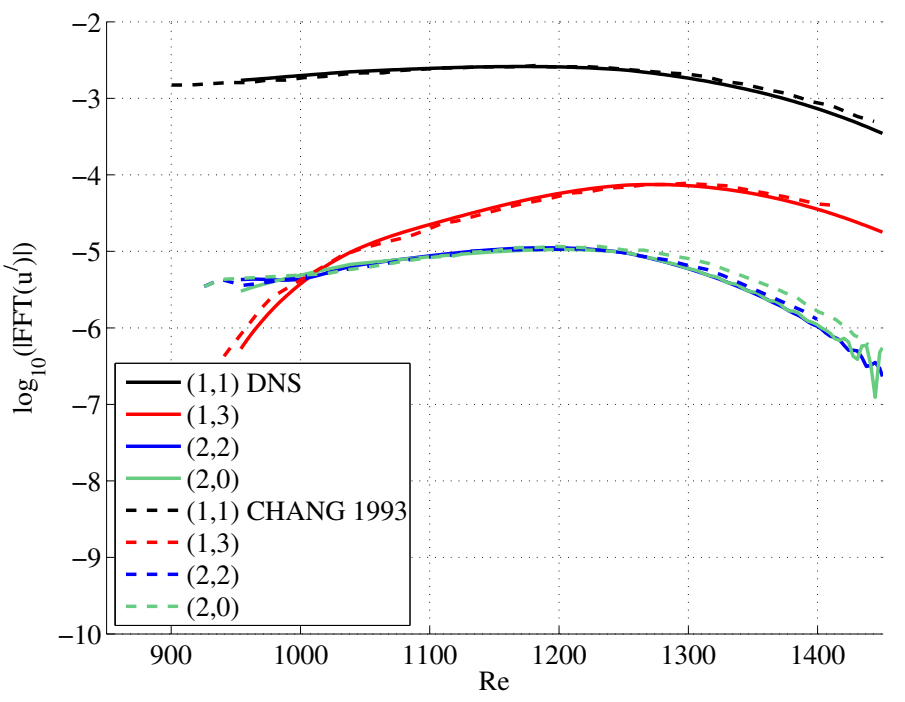

(b)

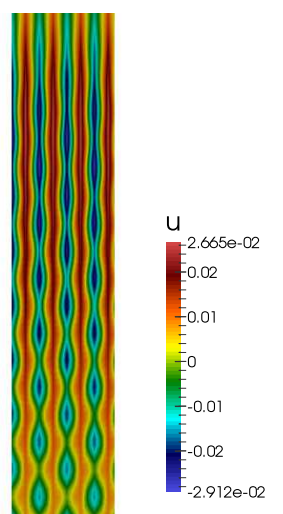

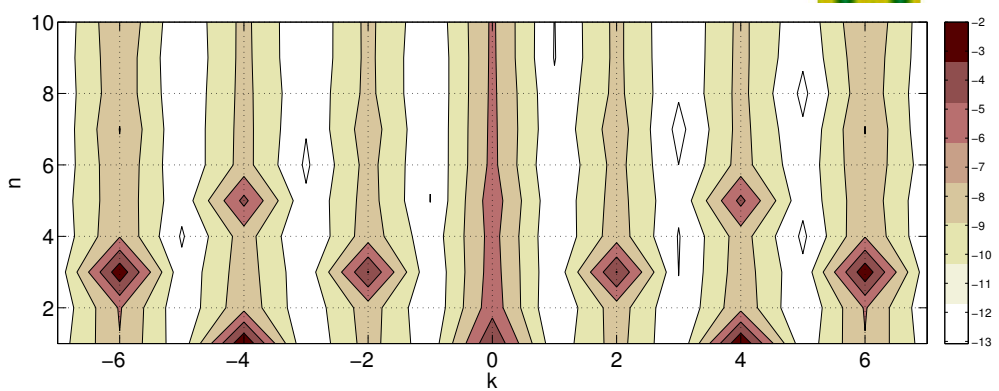

(c)

Figure 3.7: Comparison of amplification curves (a) for oblique transition reported in (JOSLIN; STREETT; CHANG, 1993) and calculated with DNS. (b) Streaks in xz plane (c) spectrum $\left(k \beta_{0}, n \omega_{0}\right)$ at $R e=1300$. 


\section{DNS simulation of experimental wave packet on incompressible boundary layer}

\subsection{Numerical set-up}

\subsubsection{Choice of reference experiment}

In the literature, there are three main reference experiments of wave packets on incompressible boundary layer. In these experiments, the disturbance is introduced in the flow by a defined acoustic time signal driving a loudspeaker that was located at the center of the plate and communicated to the fluid via a small hole. Gaster e Grant (1975) focused on the linear evolution for comparison with the theoretical linear model proposed by (GASTER, 1975), which is valid for low amplitudes of the packet. In that experiment, the wave packet amplitude is of the order of $0.05 \%$ of the free-stream velocity, measured close to the outer amplitude peak of the Tollmien-Schlichting wave, at $y=1.1 \delta^{*}(x)$. The disturbance is a rectangular pulse in time, with amplitude and pulse width as parameters. Initial subharmonic amplification is also observed in the last measurement points.

In (COHEN; BREUER; HARITONIDIS, 1991), the evolution from the linear wave packet to the formation of turbulent spots is investigated. In the evolution the packet amplitudes varies from $0.4 \%$ to $27 \%$ of the free stream velocity measured close to the inner amplitude peak of the Tollmien-Schlichting wave at $y=0.6 \delta^{*}(x)$. The disturbance was produced by a single period of a sinusoidal wave of frequency $24 \mathrm{~Hz}$, chosen to coincide with the most linearly unstable 2D mode in the experimental arrangement. Later in (COHEN, 1994), an analysis for individual modes is carried out for the same experimental data which identifies nonlinear growth of oblique modes. In the analysis, these modes are separated in three bands of frequencies, considering as reference, the frequency of the $2 \mathrm{D}$ dominant linear mode. The first band is centered around half of the reference frequency (the subharmonic band), the second band contains modes around the reference frequency (the fundamental band) and the third band represents higher frequency modes. Results show 
strong nonlinear amplification in the three bands, however no attempt was made to find an explanation for the origin of the nonlinear fundamental modes. From DNS simulation of this experiment, (YEO et al., 2010) argues that owing to small deviations from comparisons with LST observed in some low frequency modes, the experiment does not display a fully linear region. This is probably associated with the relatively high disturbance amplitude used in the experiment.

In (MEDEIROS; GASTER, 1999b) low amplitude packets, that evolve from $\% 0.1$ to $1 \%$ of the free-stream velocity measured at $y^{*}=0.6 \delta^{*}(x)$ are tracked, to focus on subharmonic amplification, effect of phase (MEDEIROS; GASTER, 1999a) and later on amplification of modes related to the fundamental resonance (MEDEIROS, 2004). The disturbance is generated by the superposition of $N_{n}=80$ Fourier modes, using $f_{0}^{*}=5 \mathrm{~Hz}$ as the fundamental frequency, then, the unstable linear band is covered $\left(5 H z \leq f^{*} \leq 80 f_{0}^{*}\right)$ which is equivalent to $0.0013 \leq \omega \leq 0.1$. In (MEDEIROS; GASTER, 1999b) the measurement streamwise positions are between $400 \mathrm{~mm}$ and $1300 \mathrm{~mm}$, spaced by $100 \mathrm{~mm}$. The spanwise length $z_{d}^{*}$, was $0.36 \mathrm{~cm}$ with 37 measurement points.

The experimental parameters of (COHEN; BREUER; HARITONIDIS, 1991) and (MEDEIROS; GASTER, 1999b) are shown in table 1 . The excitation is located at $x_{0}^{*}$, with Reynolds number $R e$, the measurements were performed up to the maximum streamwise position $x_{\text {max }}^{*}$. The value $\% u_{R e_{1450}}$, is the disturbance amplitude at the position defined by the local Reynolds number $R e \sim 1450$, which is close to the perturbation source, and serves as an indication of the initial disturbance amplitude. For the mentioned experiments, $R e \sim 1450$ corresponds to the positions $x^{*}=1600 \mathrm{~mm}$ and $x^{*}=600 \mathrm{~mm}$ on each experiment respectively.

Both experiments have similar non-dimensional parameters, but (MEDEIROS; GASTER, 1999a; MEDEIROS; GASTER, 1999b) presents lower excitation amplitudes. The last experimental measurement points have similar local Reynolds number $R e_{\max }$. As a consequence of the amplitude choice, in (COHEN; BREUER; HARITONIDIS, 1991) turbulent spots were observed at this position, while in (MEDEIROS; GASTER, 1999b) moderate nonlinear behavior was evidenced. Some wave packet experiments have been reproduced numerically for comparison and to extend their results. (GASTER; GRANT, 1975) experiment was simulated by (KONZELMANN; FASEL, 1991; SEVERIN, 2012) while (COHEN; BREUER; HARITONIDIS, 1991) experiment was simulated by (YEO et al., 2010). A DNS simulation of the experiment (MEDEIROS; GASTER, 1999b) is not available in the literature at present. It is expected that lower excitation amplitudes are better representations of real aircraft conditions at cruise. Because of this, the experiment of (MEDEIROS; GASTER, 1999a) was chosen as a reference case for the current study. Moreover the experimental data base was available and had more information on the weakly nonlinear regimes, which is the focus of the current work. It is important to note that the actual data used is not that from (MEDEIROS; GASTER, 1999b), they comes from another very similar experiment for 
which the experimental results were available. The experimental parameters were the same, with only a small difference in the wave packet amplitudes

Table 1: Experimental Parameters

$\begin{array}{lll} & \text { Cohen Experiment (1991) } & \text { Medeiros Experiment (1999) } \\ U_{\infty} & 6.65 \mathrm{~m} / \mathrm{s} & 17.3 \mathrm{~m} / \mathrm{s} \\ x_{0}^{*} & 800 \mathrm{~mm} & 203 \mathrm{~mm} \\ \nu^{*} & 1.49 \times 10^{-5} \mathrm{~m}^{2} / \mathrm{s} & 1.49 \times 10^{-5} \mathrm{~m}^{2} / \mathrm{s} \\ \delta_{0}^{*} & 2.13 \times 10^{-3} \mathrm{~m} & 7.195 \times 10^{-4} \mathrm{~m} \\ \operatorname{Re} & 1035 & 835 \\ \operatorname{Re} & 2150 & 2114 \\ x_{\max }^{*} & 3500 \mathrm{~mm} & 1300 \mathrm{~mm} \\ z_{d}^{*} & \pm 40 \mathrm{~cm} & \pm 18 \mathrm{~cm} \\ \% u_{\operatorname{Re}_{1450}} & 0.4 \% & 0.2 \%\end{array}$

\subsection{Disturbance Generation}

Usually, in experiments and simulations of wave packets, the frequency spectrum of the perturbation is carefully controlled, while less attention is given to the spanwise spectrum, which experimentally is also more difficult to control. In the present simulations, the perturbation is constructed with the superposition of a selected band of frequencies and spanwise modes, all with the same energy, covering the linear unstable band defined by the stability diagram.

To perform the simulations, the total number of frequency modes $N_{n}=80$ was considered, and 80 symmetric spanwise wave numbers $\left(N_{k}=40\right)$, in addition to the corresponding two-dimensional mode. The time signal of the excitation was identical to that used by (MEDEIROS; GASTER, 1999b). In the physical space, the resulting signal in time and spanwise direction is a function similar to $\frac{\sin (x)}{x}$. In the streamwise direction the shape of the disturbance function is one single period of the coseno function. The perturbation is applied in the wall normal velocity component. In summary, the so called pulse excitation used in the current study is then defined as

$$
v^{\prime}=A_{3 D} \sum_{n=1}^{N_{n}} \sum_{k=-N_{k}}^{N_{k}}\left[\cos \left(\alpha_{0}\left(x-x_{0}\right) \pm k \beta_{0} z\right) \cos \left(n \omega_{0} t\right)\right]
$$

with $A_{3 D}$ the amplitude of each modes.

Using the dimensional parameters given in table 1 for the experiment (MEDEIROS; GASTER, 1999a), the non-dimensional flow parameters used in the DNS simulation of this experiment are $R e=835$ and $\operatorname{Pr}=0.71$. Although the experimental Mach number is 0.05 , in the DNS simulation Mach 0.2 was used, because by increasing Mach number the numerical time steps can be increased and a lower number of iterations are required to complete the 
simulation, while at the same time the flow remains substantially incompressible. This value has already been used in other works on transitional incompressible boundary layer (e.g (SAYADI; HAMMAN; MOIN, 2013)). We also performed tests at lower Mach numbers and demonstrated that the results of $M a c h=0.2$ are essentially incompressible. Tests also performed in this study show that at Mach 0.3 some significant compressible effects arise. With the reference parameters used in the simulation, the experimental measurement domain in non-dimensional variables is defined by:

$x_{0}=284$

$0 \leq x \leq 1300 \mathrm{~mm} / \delta_{0}^{*}=1808$

$y=0.6 \delta^{*}(x) / \delta_{0}^{*}$

$L_{z}=36 \mathrm{~cm} / \delta_{0}^{*}=500$

$f_{0}=5 H z \times\left(u_{\infty} / \delta_{0}^{*}\right)=2.0796 \times 10^{-4}$

$\omega_{0}=2 \pi f_{0} \times\left(u_{\infty} / \delta_{0}^{*}\right)=0.0013066$

$\beta_{0}=2 \pi /\left(36 \mathrm{~cm} / \delta_{0}^{*}\right)=0.0126$

From these values the computational domain was defined considering extra size dimensions to avoid boundary condition effects into the region of interest. The integration domain is:

$0 \leq x \leq 2000$

$0 \leq y \leq 20 \approx 2.58 \times \delta_{99}(x=2000)$

$-\frac{\pi}{\beta_{0}} \leq z<\frac{\pi}{\beta_{0}}$

The boundary layer thickness $\delta_{99}^{*}$ can be written in terms of $\delta_{0}^{*}$ as $\delta_{99}^{*} \approx 5 \sqrt{\frac{\nu^{*} x^{*}}{U_{\infty}}}=5 \sqrt{\frac{x}{R e}} \delta_{0}^{*}$. Test were performed to ensure that this domain was sufficiently large to represent a semi-infinite flat plate immersed in an uniform flow. In the experiment, the time signal perturbation was composed by a superposition of 80 Fourier modes with fundamental frequency $f_{0}^{*}=5 \mathrm{~Hz}$. In the non-dimensional variables, the perturbation frequency band is $0 \leq \omega \leq 80 \omega_{0}=0.10453$, the spanwise wave number band is $-40 \beta_{0} \leq \beta \leq 40 \beta_{0}$, equivalent to $-0.504 \leq \beta \leq 0.504$.

In the computational domain, the perturbation has a format given by one period of the coseno function, $\cos \left(\alpha_{0}\left(x-x_{0}\right)\right)$, with $x_{0}=284$ the center of the perturbation. The value $\alpha_{0}=2 \pi / 32$ was chosen to have similar streamwise wave number of the most linearly unstable mode in the Reynolds number range studied. Figures 4.1 and 4.2 show the perturbation in physical and Fourier space.

Variables of DNS uses global nondimensional variables, however, in the instability analysis sometimes it is important to consider local nondimensional variables, using as reference length the local displacement thickness $\delta^{*}(x)$. The local Reynolds number $R e_{\delta^{*}(x)}$, local 
frequency $\omega_{\delta}$ and local spanwise wave number $\beta_{\delta}$ are related to the global ones by:

$$
R e_{\delta^{*}(x)}=1.7208 \sqrt{x R e}, \quad \omega_{\delta}=\sqrt{\frac{x}{x_{0}}} \omega, \quad \beta_{\delta}=\sqrt{\frac{x}{x_{0}}} \beta
$$
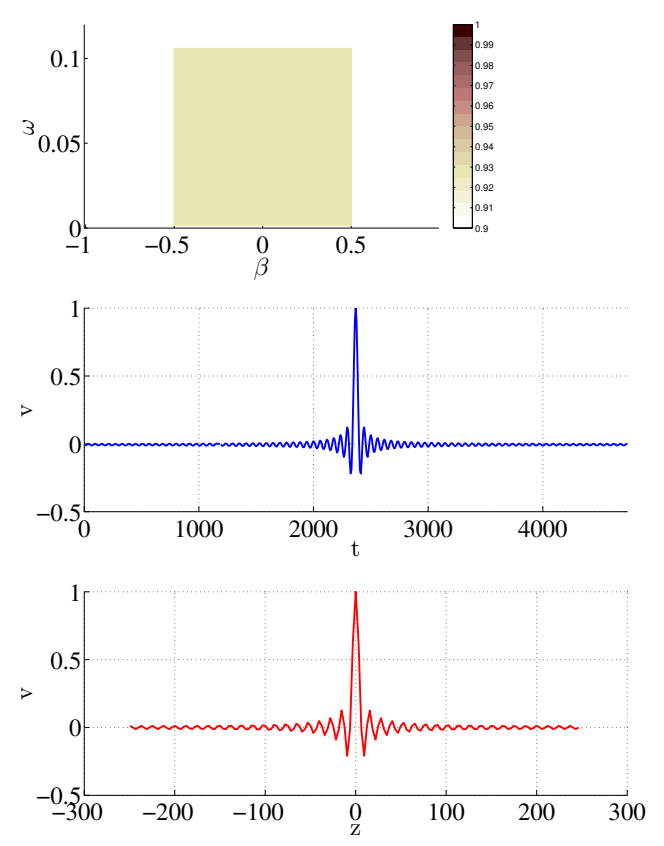
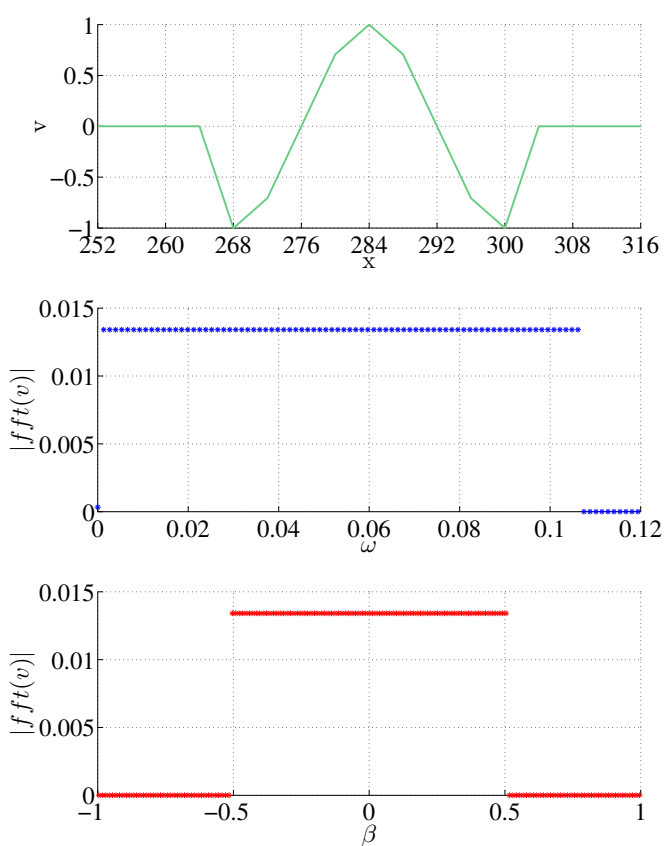

Figure 4.1: Perturbation composition, (a) two-dimensional spectrum, (b) format in streamwise direction, (c) and (d) time signal and time spectral composition. Spanwise perturbation shape in physical space (e) and spectrum (f ).

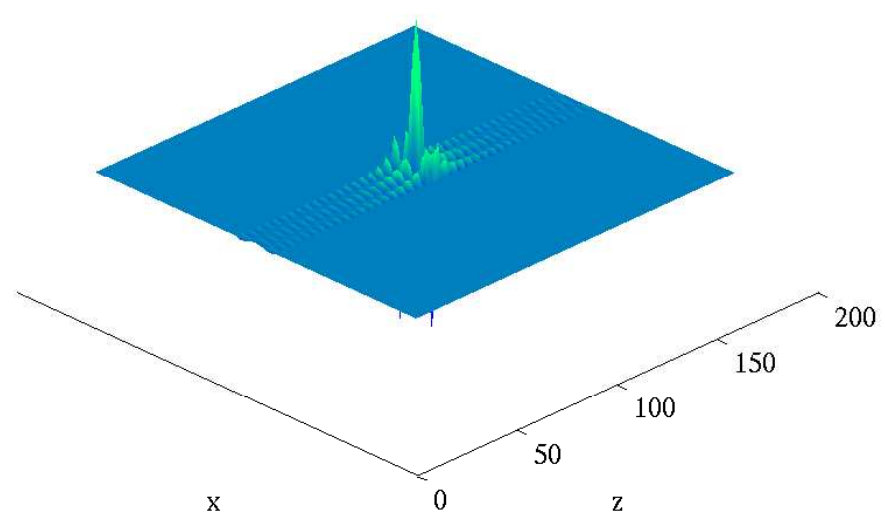

Figure 4.2: Perturbation format in $\mathrm{x}-\mathrm{z}$ plane. 


\subsubsection{Grid independence tests}

Grid resolution in $\mathrm{x}$ and $\mathrm{z}$ direction are more demanding for nonlinear than for linear regime of transition in boundary layer (CHRISTIAN; J.; SIEGFRIED, 2000). In the streamwise direction three grid resolutions were tested for wave packet simulations, namely, $\Delta x=4, \Delta x=2$ and $\Delta x=1$. Tests revealed significant grid dependence in the the nonlinear regime for $\Delta x=4$. In the figure 4.3 wave packets with two grid resolutions in streamwise direction are plotted. With $\Delta x=2$ the results are fairly grid independent up to later stages of the weakly nonlinear regime, which is the focus of the current work. In the following simulations, a grid with $\Delta x=1$ was employed for the calculations. A more refined grid was not possible to simulate with our current computational resources, due to high computational and storage cost. The refinement in the wall normal direction tests were performed for TS-waves in the defined computational domain (see figure 4.4), and lead to the conclusion that for $y_{\max }=20$ and $n y=51$ the results are independent of grid and domain extent. In the spanwise direction, the resolution of 4 points per-wavelength was sufficient for grid independence (figure 4.5). The grid employed in the wave packet simulations was $n x=2001, n y=51, n z=160$. The value of $n z$ was chosen to provide resolution of four points per wave length in the mode $40 \beta_{0}=0.504$, which is the higher spanwise wave number captured in the experiment, but which was above the highest spanwise wave number observed in the DNS results.

(a)
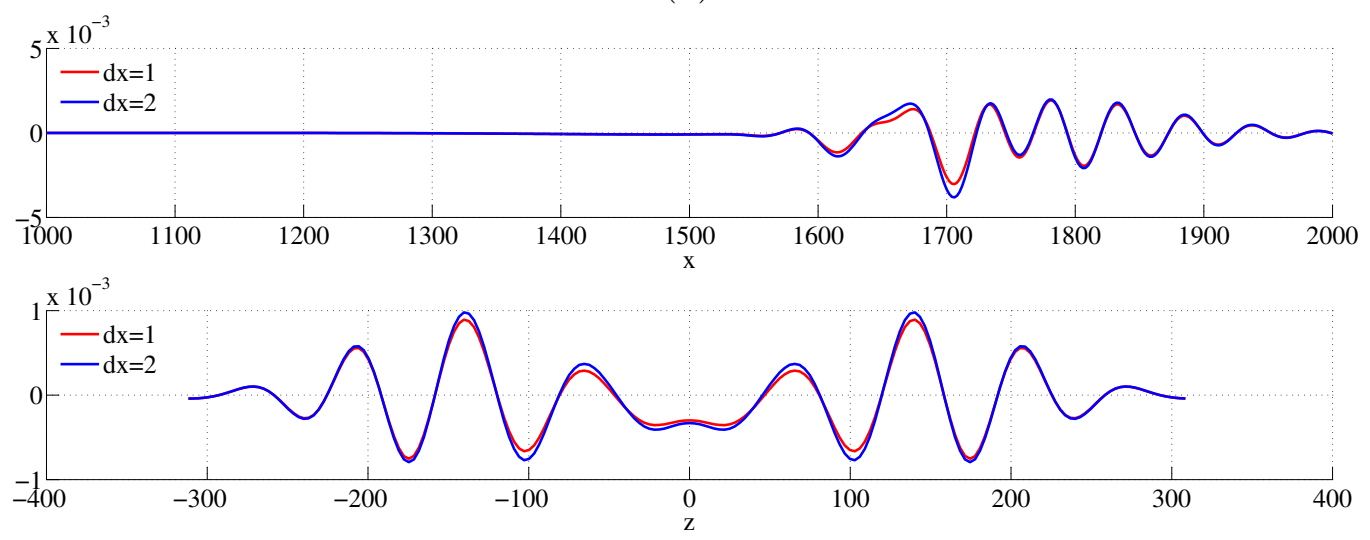

(b)

Figure 4.3: Results for grid independence in (a) streamwise and (b) spanwise directions in the nonlinear regime of the wave packet. 


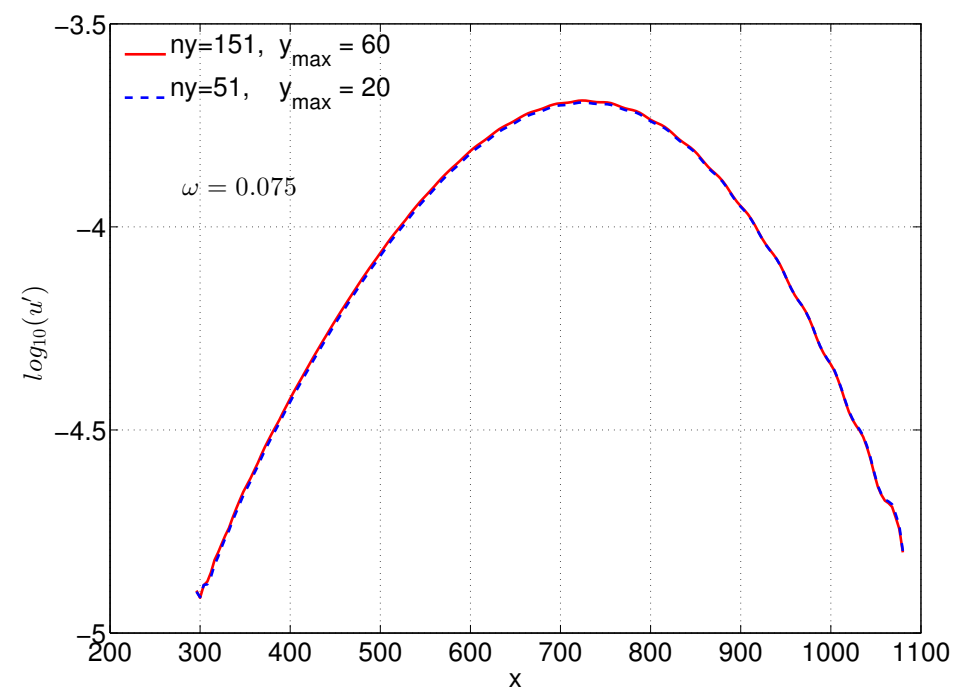

Figure 4.4: Test for grid independence in y-direction for a TS-wave in the computational setup for the experiment simulation, with $\omega=0.075$.

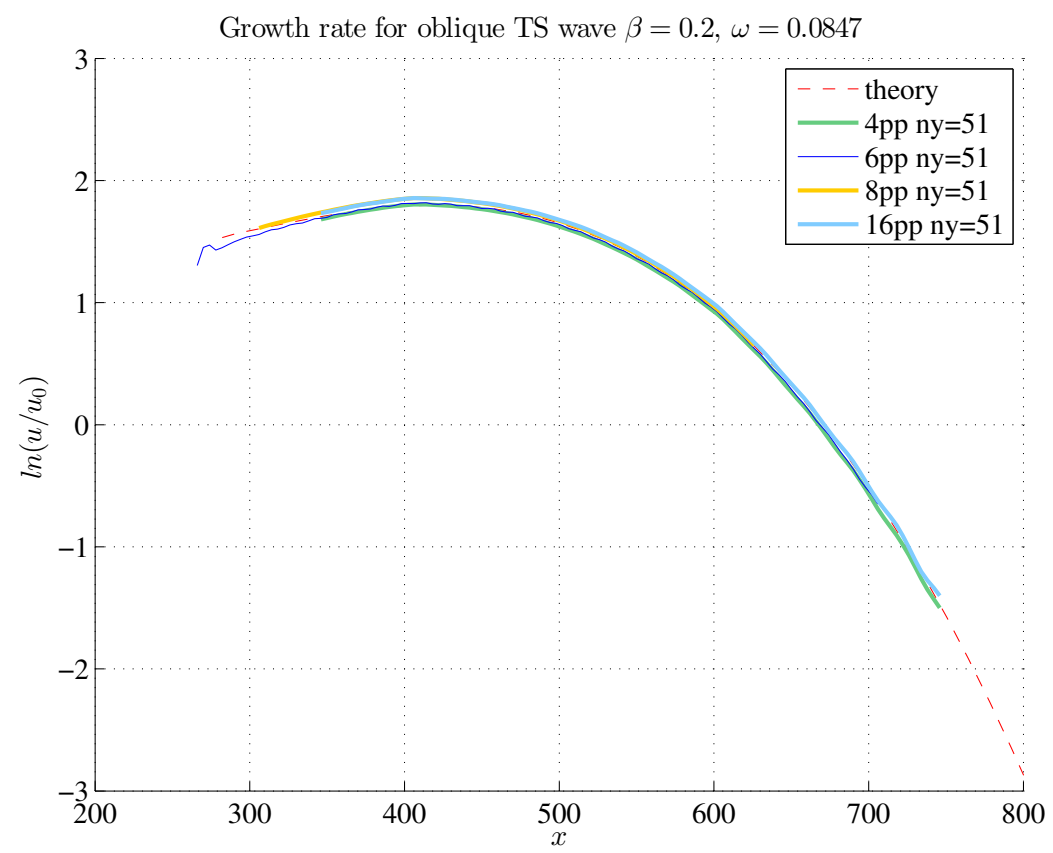

Figure 4.5: Grid independence tests in the spanwise direction for an oblique wave defined by $\omega=0.0847$ and $\beta=0.2$. The minimum resolution required in z-direction is 4 points per period of a wave with wavelenght $\lambda_{z}=\frac{2 \pi}{\beta}$. 


\subsection{DNS results}

This section is organized as follows. First a small amplitude packet was simulated to produce a linear evolution of a packet to serve as a reference for the nonlinear studies that followed. This constitutes the next subsection. Then the experiments of (MEDEIROS; GASTER, 1999a) were reproduced numerically to demonstrate that the simulations were a good representation of the actual physical observations. Next, the numerical results were analyzed in detail to try to establish conclusions regarding the nature of the nonlinear regime observed and the possible differences from the classical scenarios of unmodulated waves, such as one 2D wave in combination with a pair of small amplitude oblique waves or secondary instability mechanisms, here-called controlled transition. Finally possible causes for some small differences between the numerical and experimental results were investigated.

\subsubsection{Linear wave packet}

To perform the analysis of the wave packet evolution, Cohen (1994), Yeo et al. (2010) used predictions of a linear model and LST to compare the evolution of isolated modes in the nonlinear packet. For the present analysis, a low amplitude (linear) packet was employed as reference case. It takes into account effects as non-parallelism and very weak influence of nonlinear terms of the Navier Stokes equations, hence, it may be a closer representation of a linear wave packet generated experimentally. The evolution of the linear wave packet is shown in figure 4.7. Perturbation amplitude is of the order of $10^{-5}$. In incompressible boundary layer the packet displays the characteristic crescent shape with weak modulation in spanwise and streamwise directions. Results in the spectral space can also be obtained, and are discussed in the next section (figure 4.10 (a)), together with results for the nonlinear wave packet. In the global nondimensional variables, the frequency of the most amplified mode decays downstream, as can be seen in figure 4.11.

\subsubsection{Comparison of numerical with experimental wave packet}

To reproduce numerically the experimental results of hydrodynamic instability, it is crucial to employ the excitation amplitude that generates a wave packet with the same amplitude in a linear position of the experiment. Experimentally, the linear stages are observed up to $x^{*}=600 \mathrm{~mm}$. For the nonlinear simulation, the excitation amplitude was adjusted to match the experimental results at this location. In figure 4.6, at the experimental measurement points, the streamwise velocity time signal at the centerline is compared with the numerical results. The agreement is remarkable, in particular in view of the very small amplitudes of the signals, with maximum amplitudes about $0.1 \%$ of the free stream velocity. Significant differences are found only in the last positions, where strong 
nonlinear action takes place. Yet, even at these stations the agreement can be considered good in general.

Figures 4.7 and 4.8, show the linear and nonlinear evolution in physical space on planes parallel to the wall. A weak three-dimensional structure becomes stronger as the nonlinear effects increase downstream. Differences between linear and nonlinear regimes are more clearly shown in figure 4.9 .

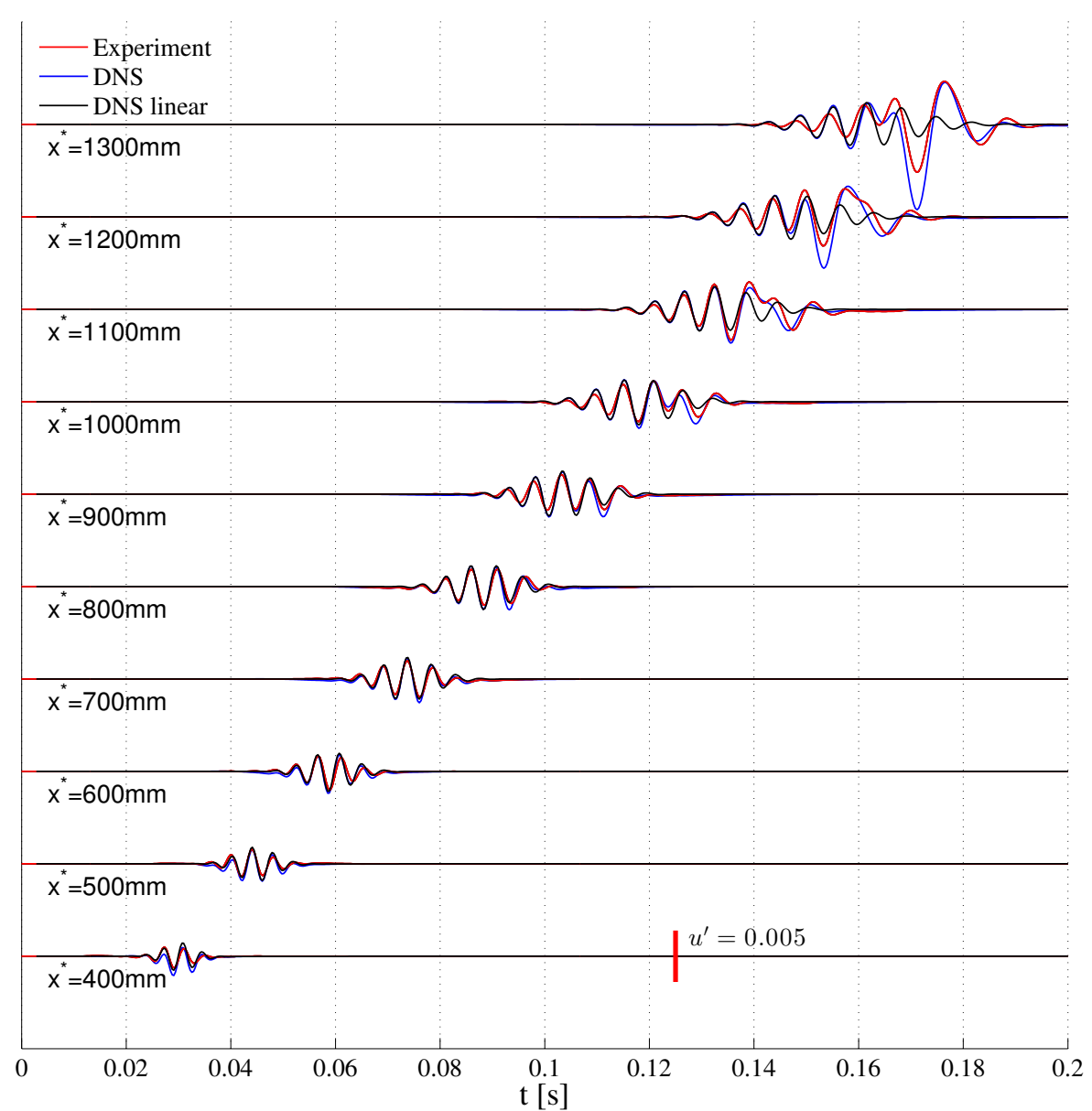

Figure 4.6: Comparison of velocity time signal at the centerline, at all experimental measurement points of (MEDEIROS; GASTER, 1999a) with DNS simulation. 


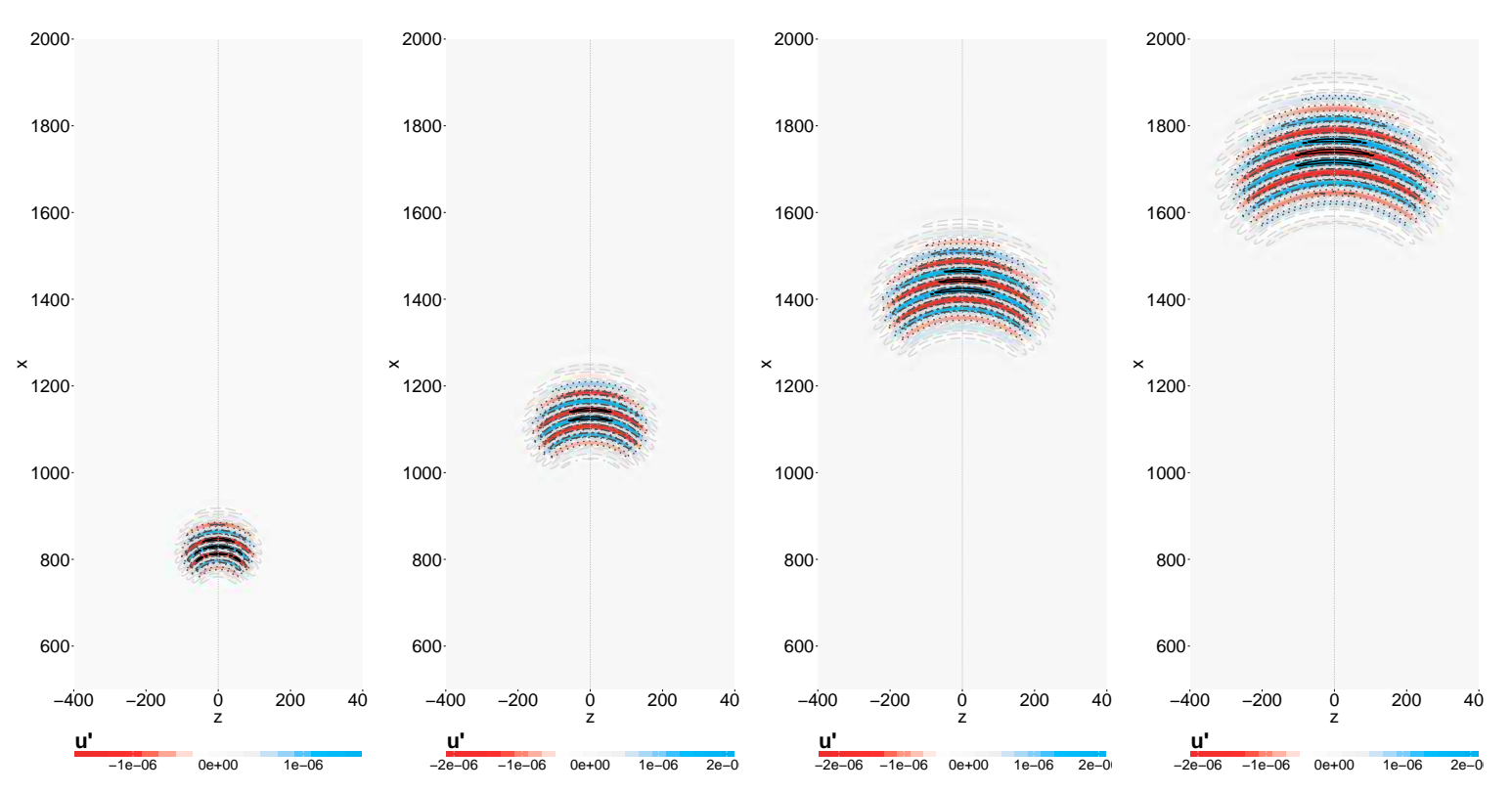

Figure 4.7: Linear wave packet at Mach 0.2 , in $y=0.6 \delta^{*}(x)$. Contour levels at $90 \%(-)$ $, 50 \%(-.-), 30 \%(\ldots)$ and $10 \%(--)$ of the amplitude peaks in the packet.

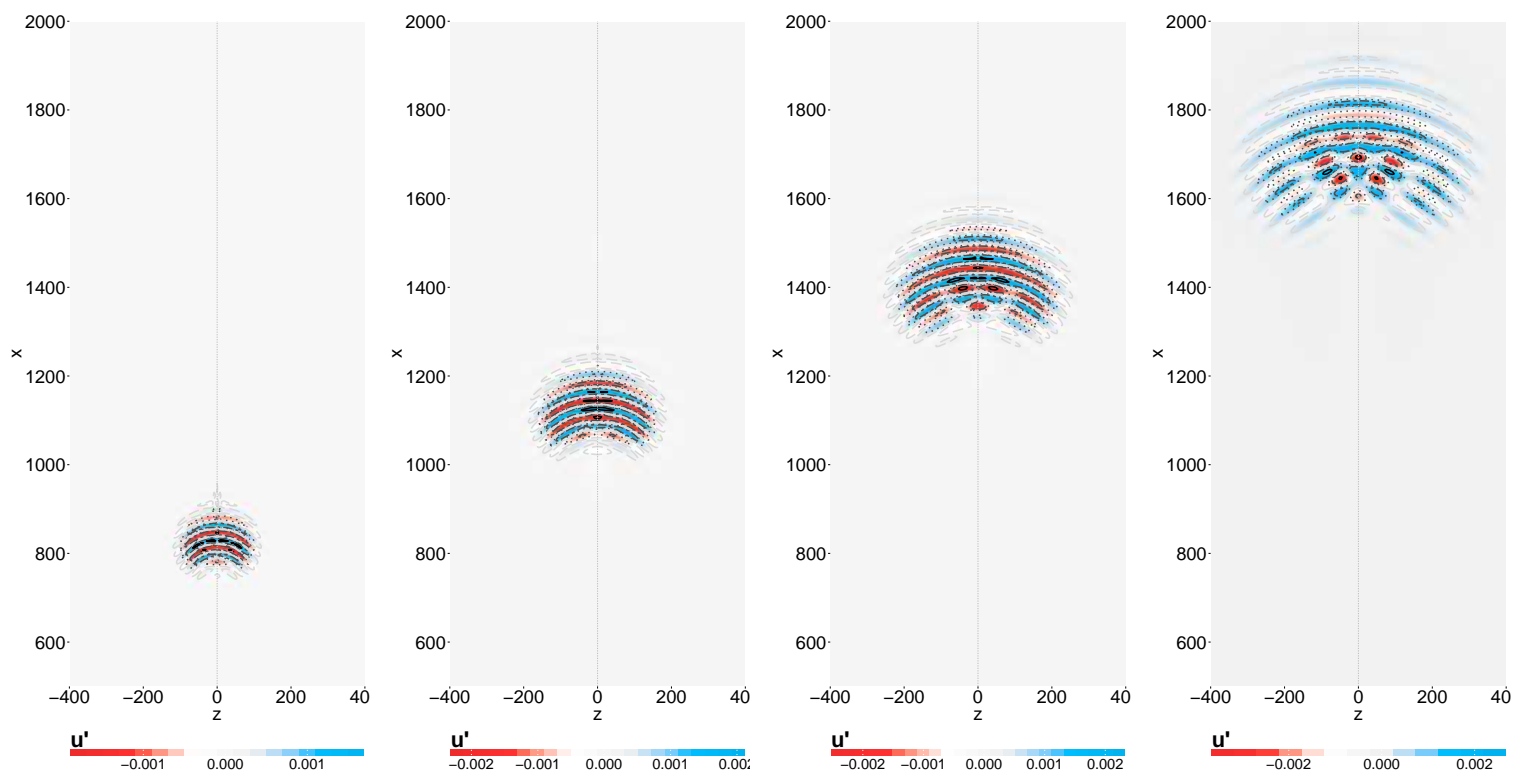

Figure 4.8: Nonlinear wave packet evolution at Mach 0.2 , at $y=0.6 \delta^{*}(x)$. Contour levels at $90 \%(-), 50 \%(-.-), 30 \%(\ldots)$ and $10 \%(--)$ of the amplitude peaks in the packet.

In figure 4.10 the spectral evolution is compared for DNS linear, DNS nonlinear and experimental wave packet, considering local nondimensional variables, using as reference length $\delta^{*}(x)$. More quantitative comparisons are provided in figure 4.12 at $x^{*}=1200 \mathrm{~mm}$. Comparison of the spectrum obtained from DNS simulation and experimental data, shows in general, good agreement. However, there are two significant differences, namely, an experimental asymmetry not present in the simulations due to the numerical formulation 
considered and a weaker nonlinear amplifications in the fundamental and low frequency modes, observed in the simulation. These differences will be addressed later in the analysis.

An important aspect in performing the analysis is that for direct comparison with the experimental results, the $\mathrm{u}$ component of the velocity calculated by the DNS simulation, was interpolated into the experimental measurement points defined in section 4.2 , by using a spline method.

The computational domain has a larger size and higher spatial resolution in the three coordinates. The interpolation was done only for direct comparison with the experiment, for subsequent analysis the DNS domain and grid resolution was used.

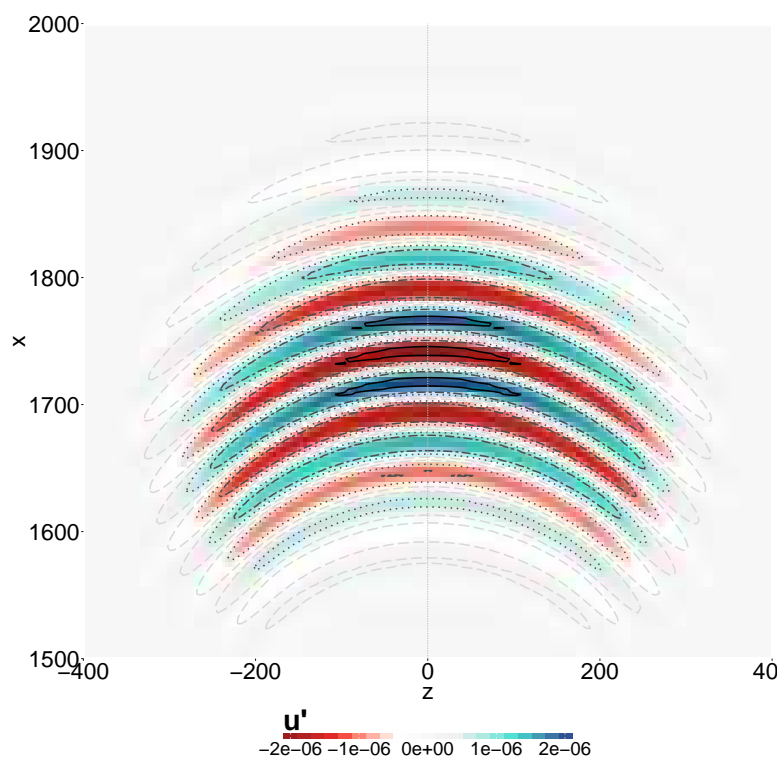

(a)

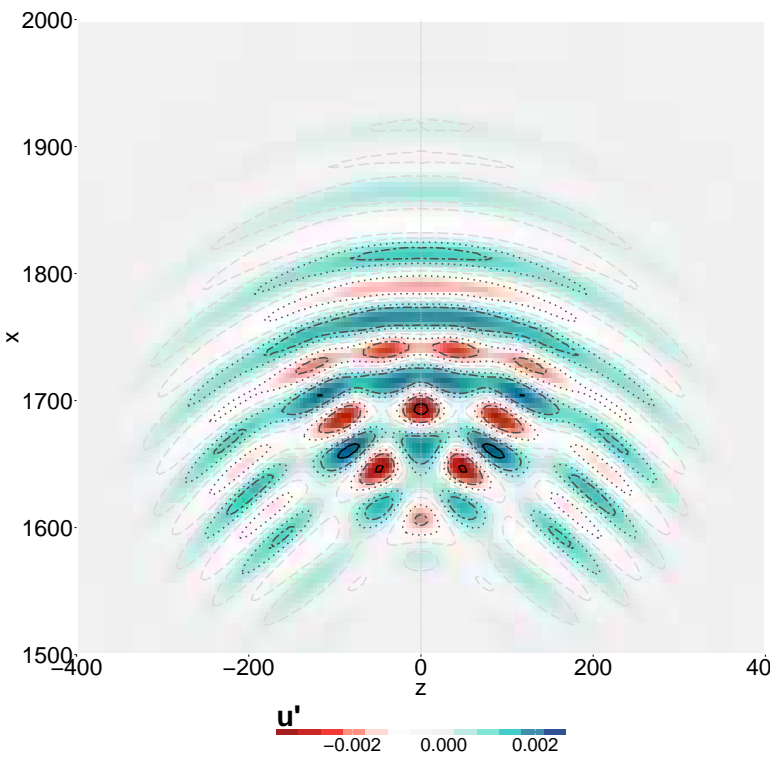

(b)

Figure 4.9: Comparison of linear (a) and nonlinear (b) wave packet at advanced position at $y=0.6 \delta^{*}(x)$. Contour levels at $90 \%(-), 50 \%(-.-), 30 \%(\ldots)$ and $10 \%(--)$ of the amplitude peaks in the packet. 

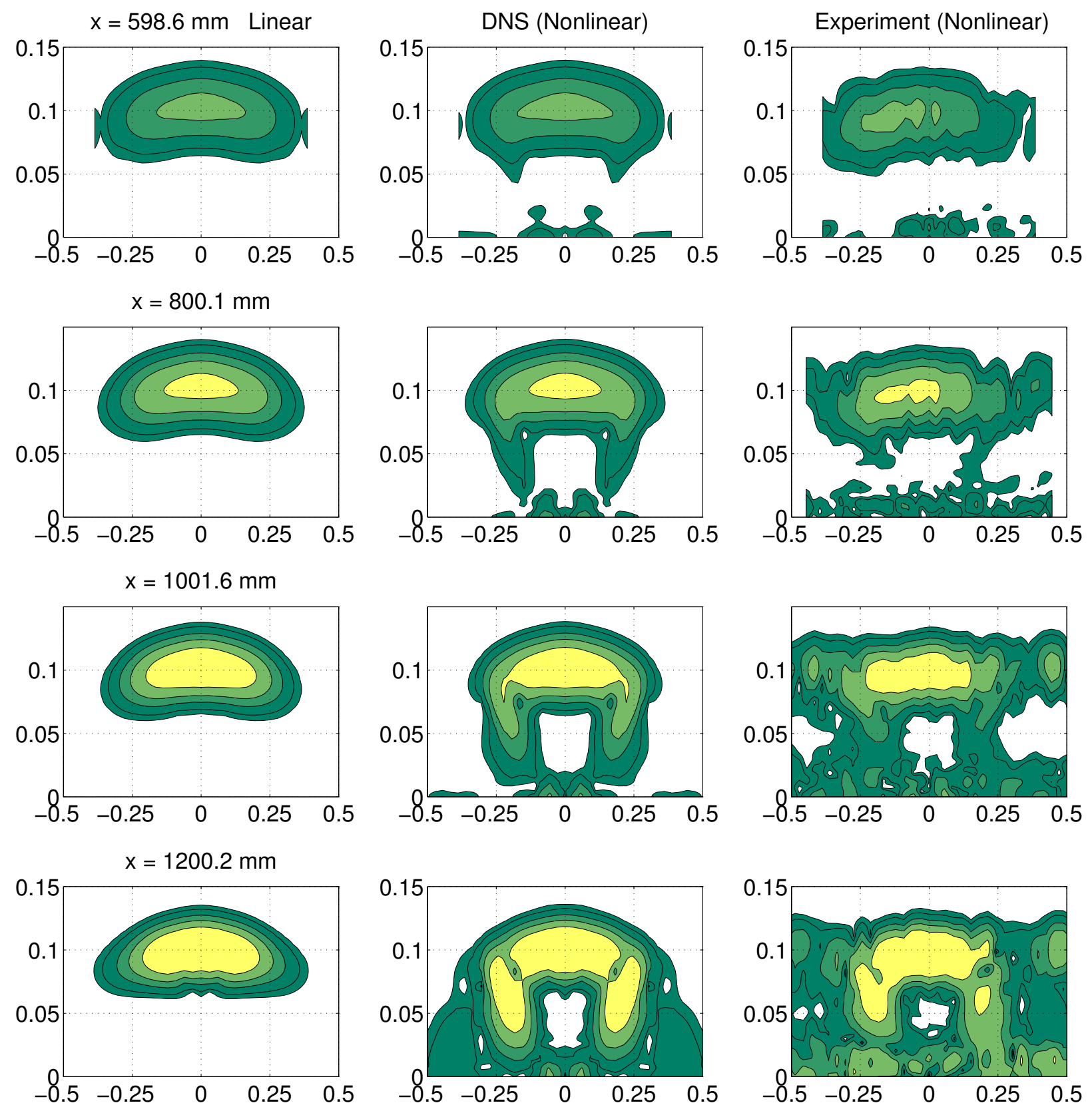

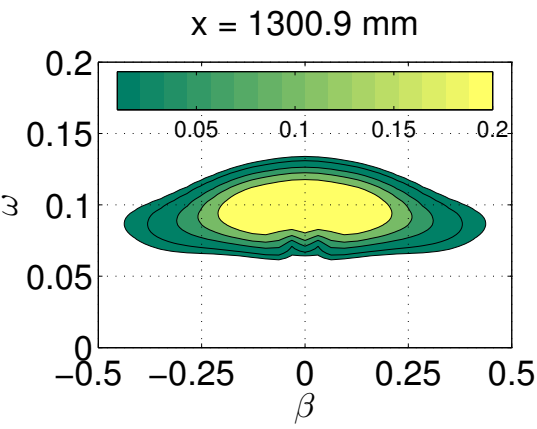

(a)

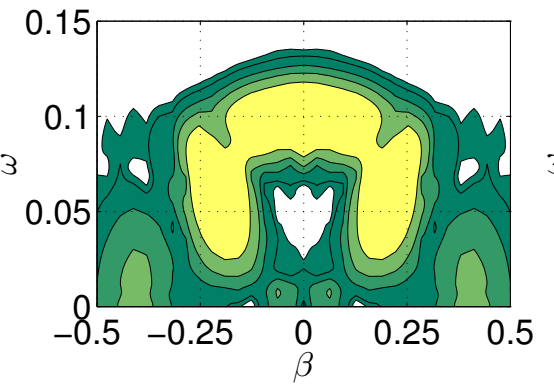

(b)

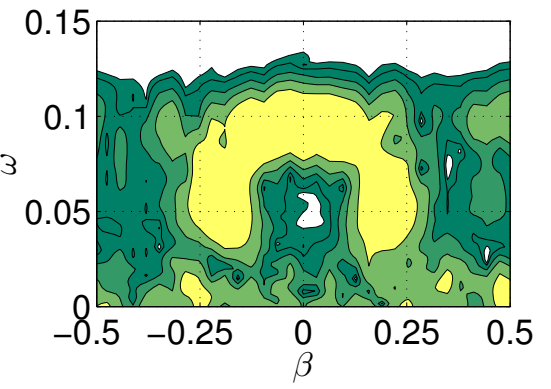

(c)

Figure 4.10: Comparison of spectral evolution at several measurement points for (a) linear DNS (b) nonlinear DNS and (c) experimental wave packet. 

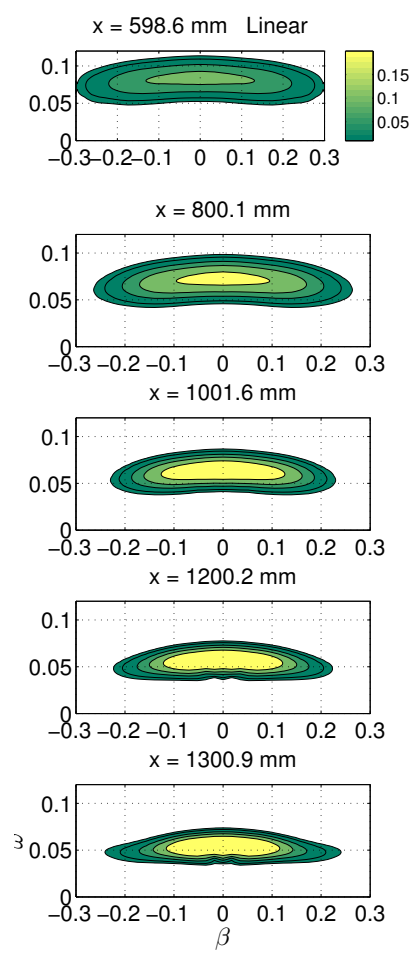

Figure 4.11: Spectrum evolution of the linear wave packet in global nondimensional variables. The frequency of the most amplified mode decays in downstream.
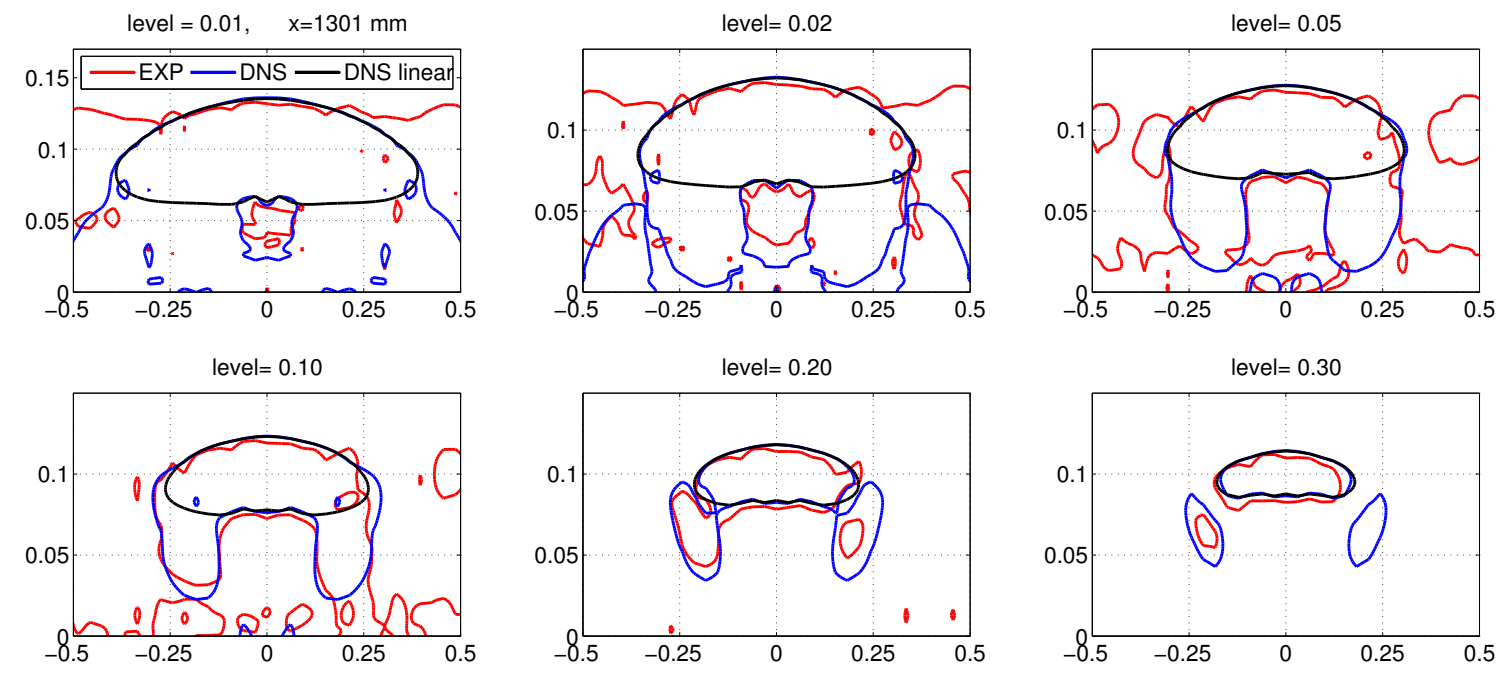

Figure 4.12: Comparison of contour levels of linear, DNS and experimental spectrum in the nonlinear position, $x^{*}=1200 \mathrm{~mm}$. 
The interpolation of numerical results into the experimental physical domain (figure 4.13), suggests that the experimental domain was a little too small in the spanwise direction, because in the interpolated DNS results (figure 4.13(a)) the wave packet is not totally contained in the spanwise direction, as is observed in the wave packet shown in the figure $4.9(\mathrm{~b})$.

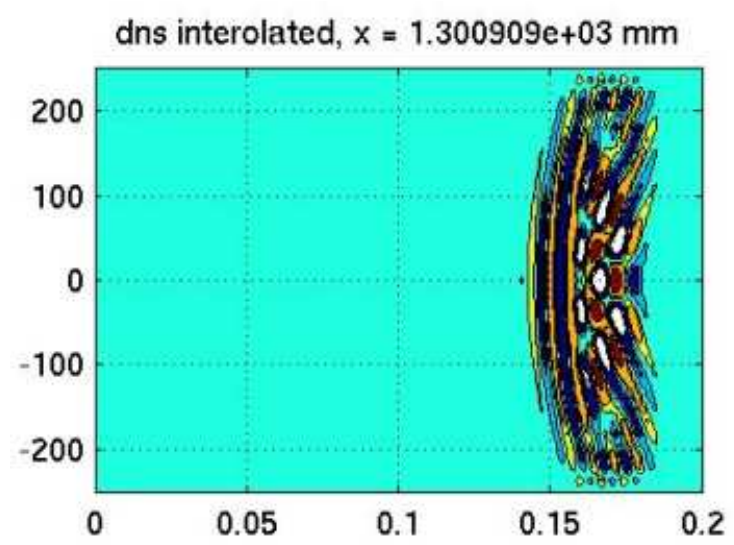

(a)

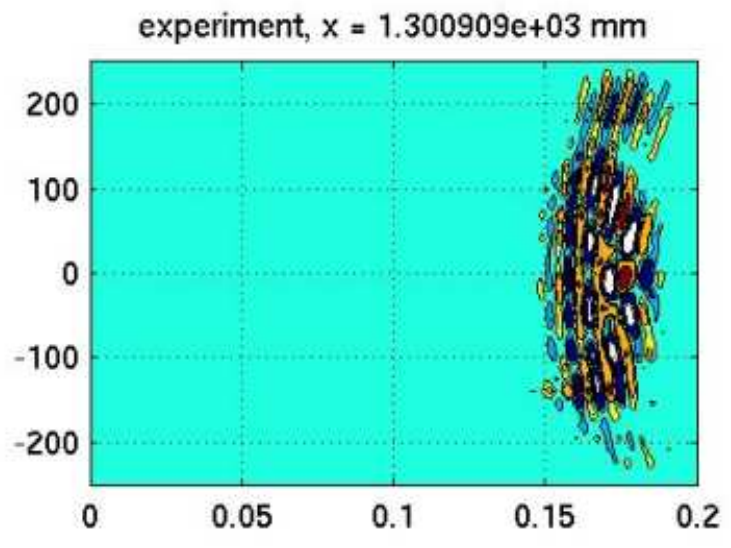

(b)

Figure 4.13: Comparison of $u^{\prime}$ component of the disturbance velocity in the experimental domain at $x^{*}=1300 \mathrm{~mm}, y=0.6 \delta^{*}(x)$. (a) DNS results interpolated at the experimental domain, (b) Experimental results. 


\subsection{Nonlinear modal analysis of DNS results}

In the nonlinear regime it is possible to distinguish four important types of modes, here called linear, subharmonic, fundamental and low frequency modes, as illustrated in figure 4.14. The subsequent analysis investigates nonlinear bands separately.

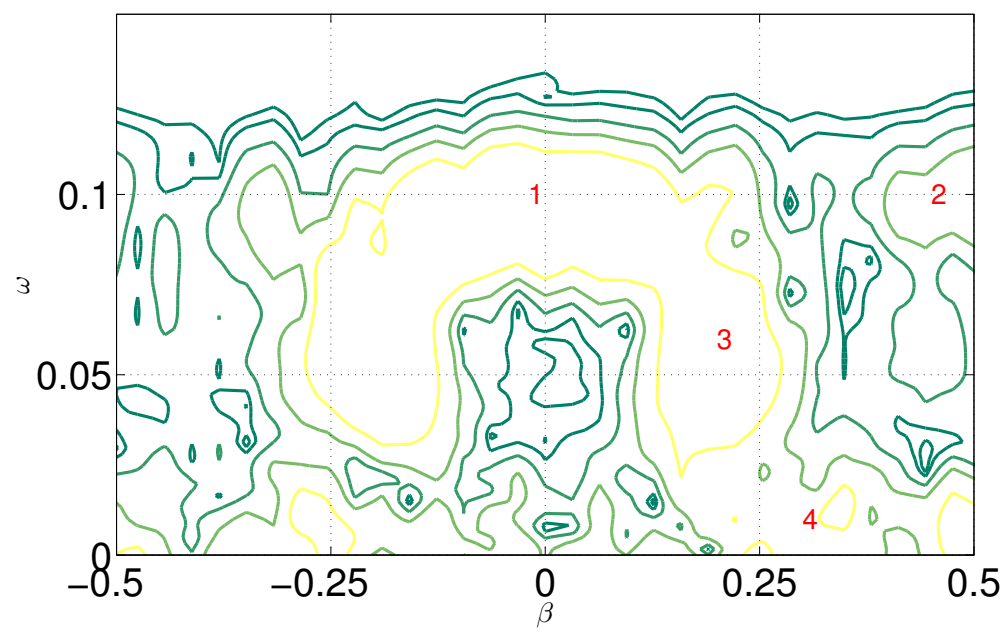

Figure 4.14: Definition of mode bands used for the analysis. (1) linear (2) fundamental (3) subharmonic and (4) low frequency bands.

\subsubsection{Subharmonic bands}

Amplification of the modes in the subharmonic band is the focus of the present section. In figure 4.15, the frames on the left column display amplitude evolution of isolated modes in the packet. The subharmonic modes displayed are indicated in the frame of the right column by the circles in the contour plots of the wave packet spectrum at position $x^{*}=1200 \mathrm{~mm}$. A mode maintain a constant dimensional frequency along the evolution, while results of hydrodynamic instability in the boundary layer are normally plotted in non-dimensional variables based on local boundary layer parameters, which, hence, change along the evolution of a mode. To emphasize that the evolution shown holds for modes, the modes are identified by their spanwise wave number index $k$ and frequency index $n$, which, for the signal processing used, remain the same along the evolution of the mode. The selected subharmonic modes are shown in two groups, one displayed in the top frames the other in the bottom frames. In the frames showing the amplitude curves, the thick continuous blue line represents the fundamental 2D mode with twice the frequency of the subharmonic band. This would be the linearly unstable mode driving a possible subharmonic resonance that is investigated. The red dashed lines correspond to the nonlinear evolution of the subharmonic modes. The dashed green lines correspond to the linear evolution of the same subharmonic modes, obtained from the above discussed linear simulation that was performed for reference. Clearly, the subharmonic modes display a 
nonlinear behavior that renders them substantially more unstable than in their linear evolution. Several modes that are linearly stable display nonlinear instability.
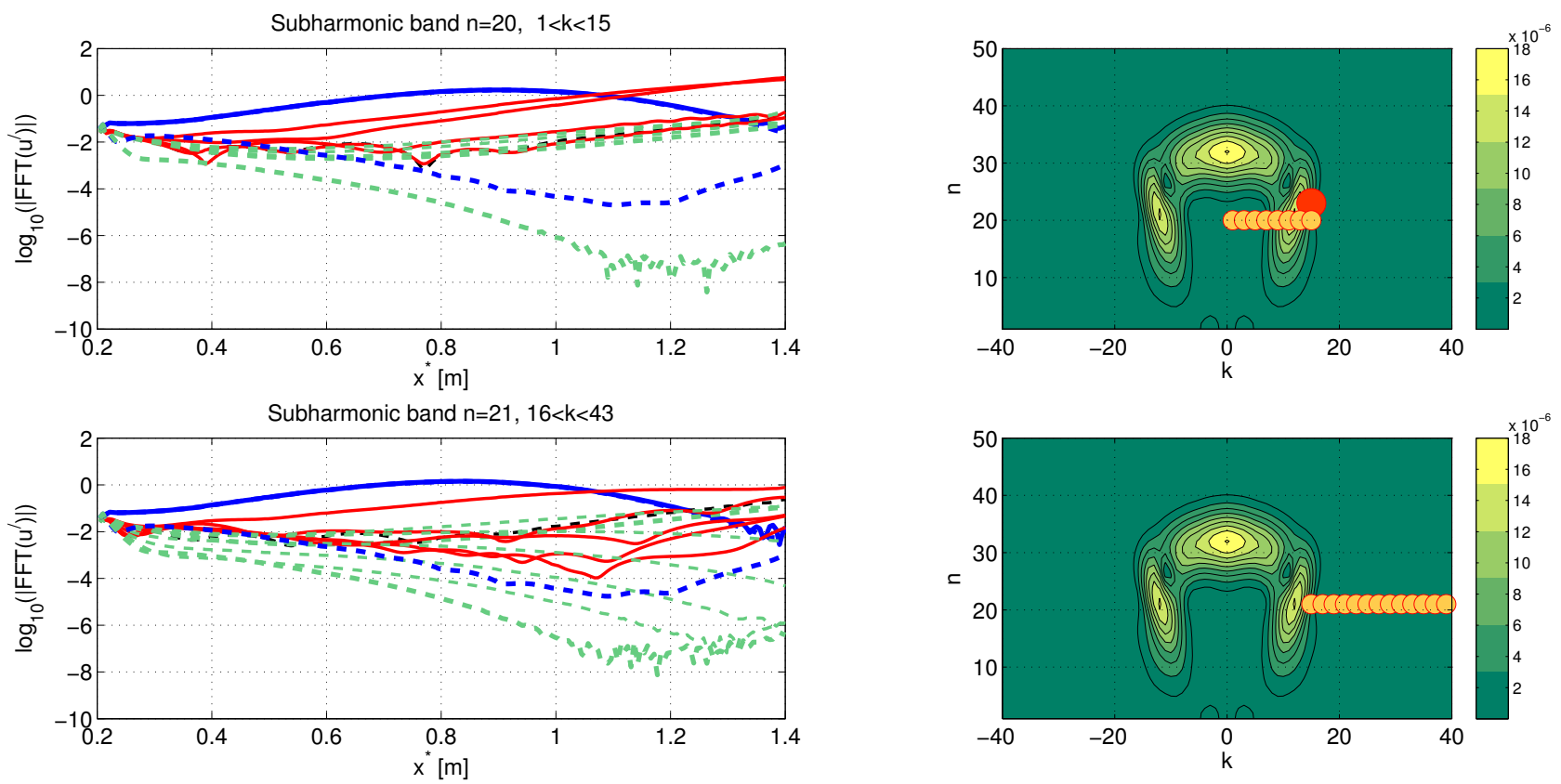

Figure 4.15: Amplification curves for subharmonic bands of linear (green lines), nonlinear (red lines) and fundamental (blue lines) modes. The evolution of the mode indicated by the red point is shown in the figure 4.16.

(a)
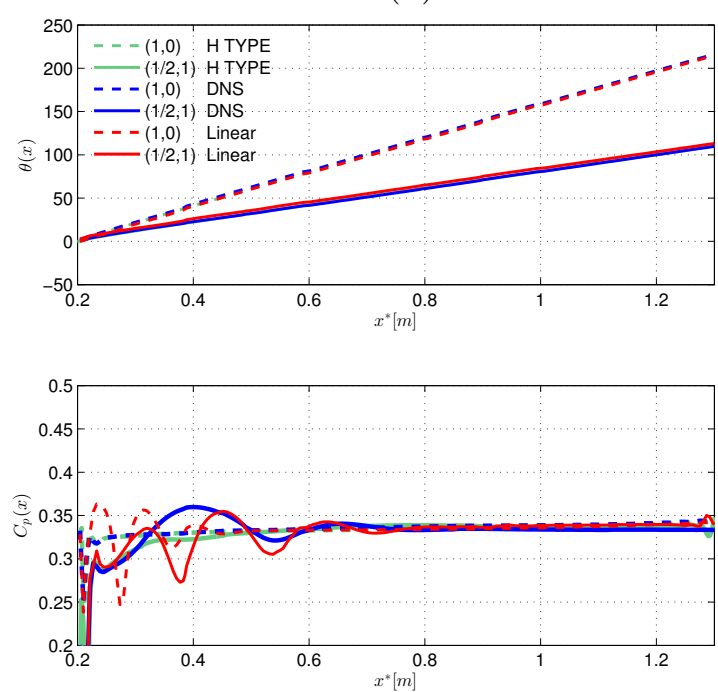

(c) (b)
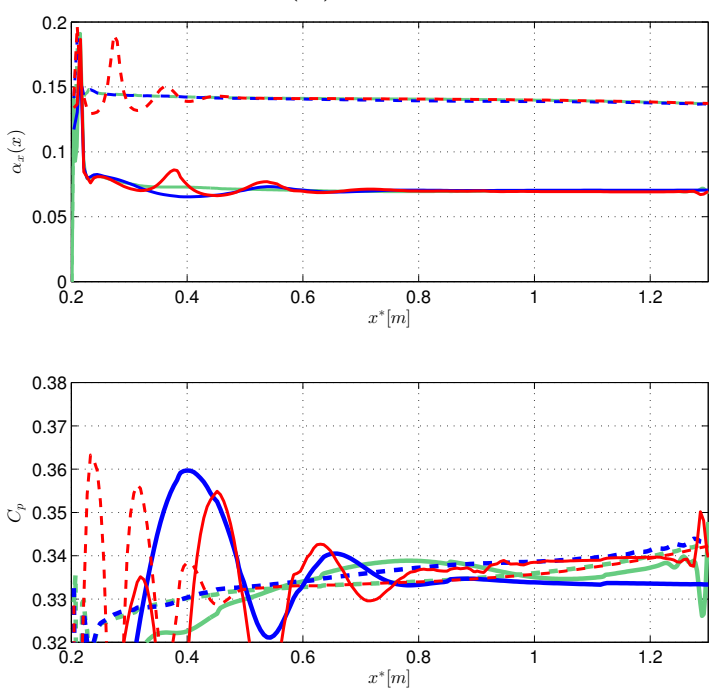

(d)

Figure 4.16: (a) Phase, (b) streamwise wave number, (c) phase velocity for subharmonic resonance, (d) zoom of (c), for the mode $\omega / 2=0.059260, \beta=-0.2218$, chosen from experimental spectrum at $x^{*}=1300 \mathrm{~mm}$. 
Sivasubramanian e Fasel (2014) investigated the phase-locking of subharmonic waves and its respective primary in a wave packet at Mach 6. In their results, phase locking was observed only in the nonlinear regime and was used as an indication of subharmonic instability. In figure 4.16 a similar analysis is presented for the current results. Figure 4.16(a) shows the phase variation of a number of modes along the $\mathrm{x}$ direction. Dashed lines and solid lines correspond respectively to the primary and subharmonic mode indicated by the red point in figure 4.15. Numerical results are shown for linear packet, nonlinear packet and controlled transition, all with good agreement. It shows clearly that no significant phase adjustment is caused by nonlinerity. That is expected for subharmonic instability of the C-type where the waves that resonate already match the phase speed required for resonance. Indeed, this secondary instability is a limiting case of H-type instability as the driving wave amplitude decays.

Conclusions are further supported by the distribution of streamwise wave number of these modes along the streamwise direction, figure 4.15(b). Some irregularities are observed at the earlier and later stages, but the bulk of the distribution is also not affected by nonlinearity. Figures 4.16(c) and 4.16(d) show the actual phase velocities, with figure 4.16(d) showing that, for a large portion of the evolution, the phase speeds of the resonant modes match each other only a little better under resonance. Once more the results are consistent with subharmonic instability of a small amplitude driving wave.

As discussed in the literature review, the subharmonic instability in a wave packet is an issue not entirely settled, with several aspects deserving more definite conclusions. What follows in an attempt to fill this gap. Nonlinear behavior of the resonant type involves phase locking. When this occurs both modes, fundamental and subharmonic reach the same phase velocity. However in the current situation, this is not clear evidence of nonlinear behavior because from beginning both modes have same phase speed, even thought this is a requirement for Craik triad resonance. This is shown in figure 4.16(c).

One important aspect in the subharmonic instability is that there is a threshold amplitude for the primary driving wave, for which instability sets is. In boundary layers, as the primary waves grow, the subharmonic growth rates increase. If the primary wave is not too large, it crosses the second branch, reaching its maximum amplitude and then decaying, while the subharmonic wave keeps growing. The subharmonic wave reaches its maximum amplitude where the primary wave reaches the threshold amplitude again, but on its decaying arm. Hence, the maximum amplitude of the subharmonic wave occurs downstream from the maximum amplitude of the primary driving wave.

Figure 4.17 helps to illustrate this concept in the wave packet. The top frame indicates the subharmonic mode tracked. For a packet in the boundary layer, the linearly unstable frequency band changes with the packet evolution. Its frequency reduces as the Reynolds number based on global parameters increases (see figure 4.11). A similar pattern may be 
observed for the subharmonic waves, although not so clearly.

For the analysis in figure 4.17, the subharmonic mode chosen was a subharmonic band peak at position $x^{*}=1200 \mathrm{~mm}$, which is a neighbor mode of the modes examined in figure 4.16. This streamwise position was chosen for clarity. Figure 4.17, bottom frame, shows the evolution of the subharmonic mode in the nonlinear packet (blue continuous line). It also shows the primary driving wave obtained from the nonlinear simulation (blue dashed line). The red dashed line is the linear evolution of the subharmonic wave, which is shown with two lines shifted in the vertical direction. The first aspect to discuss in the figure is that the primary wave is virtually unaffected by the nonlinearity. The picture indicates further that the nonlinear subharmonic wave departs from its linear behavior at a position about $x^{*}=0.25 \mathrm{~m}$. The picture also shows that at some position downstream the nonlinear subharmonic wave settles back to its linear behavior. The amplitudes of the primary wave at which the subharmonic departs from linear behavior and returns to it are also indicated in the figure, and they are virtually identical. Overall the results convey the idea of a nonlinear process of a subharmonic wave which is governed by the amplitude of the primary wave and has a well defined threshold amplitude. Moreover the primary wave remains unaffected by the nonlinear activity. The observations are consistent with a subharmonic instability driven by a small amplitude primary wave. It is interesting to mention in passing that the subharmonic wave does not reach its maximum amplitude at the decaying threshold point, owing to the fact that at that $\mathrm{x}$ location the nominally subharmonic waves have already become linearly unstable. Sometimes, in the literature the pair of resonating modes are identified by their peaks in the spectrum in a fixed position. However, as explained before, the peaks of the fundamental and subharmonic waves must normally occur at different streamwise positions. This fact incorrectly suggest the presence of detuned subharmonic resonance.

Figure 4.18 shows the amplitude evolution of the primary and subharmonic modes indicated in the two-dimensional spectrum by the red points, calculated in the experimental domain. The amplitude evolution is given for experimental (read lines) and nonlinear numerical (blue lines) results, and they agree very well with one another. Amplification curves also are compared with the corresponding linear evolution (black line). Once more, the primary wave is unaffected by nonlinearity while the subharmonic wave becomes nonlinear very early in its evolution. The green lines represent the subharmonic resonance induced by controlled transition, meaning, a two dimensional wave with the frequency of the primary wave and a pair of subharmonic waves with the same frequency and spanwise wave numbers of the other subharmonic waves. The amplitude of the primary wave in the controlled transition was chosen to match the nonlinear subharmonic wave growth rate in the packet. Indeed the matching is fairly good, and indicates that the phenomenon observed in the packet corresponds to subharmonic resonance of a primary wave at some 
amplitude. The amplitude of such primary wave is not that of the $2 \mathrm{D}$ fundamental wave composing the packet.

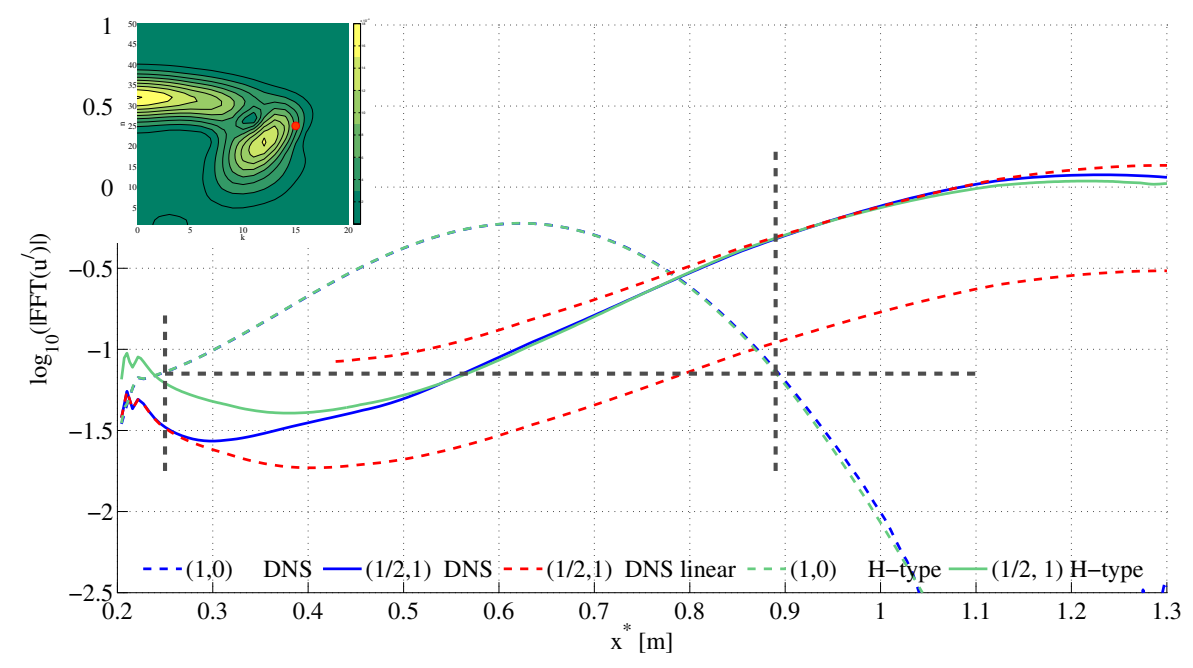

Figure 4.17: Amplification curves for the subharmonic mode $(25,15)$ and its fundamental mode. The threshold amplitude of the driving mode $(1,0)$ (indicated by the gray dashed lines) catalyses the subharmonic amplification by increasing the growth rate. The nonlinear growth rate for the mode $(1 / 2,1)$ calculated from H-type controlled transition, matches with the growth rate for the same mode in the packet.
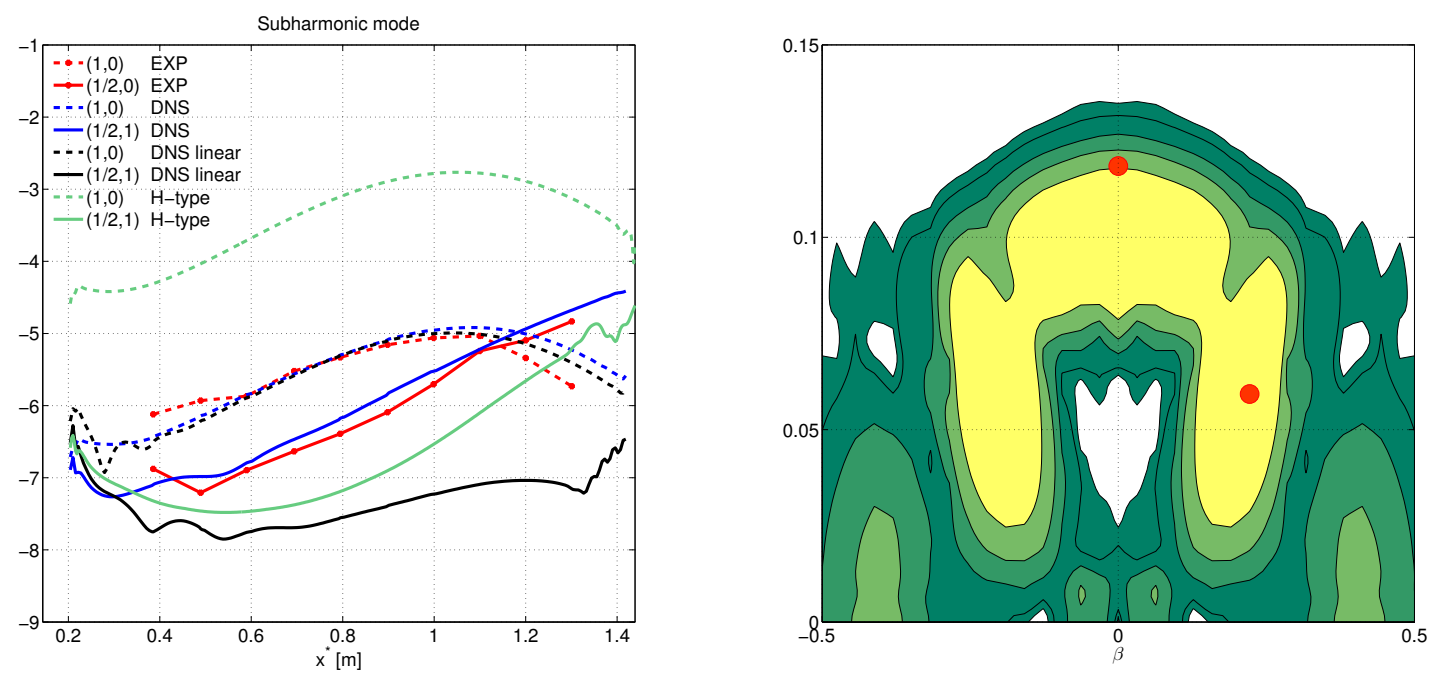

Figure 4.18: Amplification curves of the most amplified subharmonic in the experimental domain at $x^{*}=1200 \mathrm{~mm}$.

Figure 4.19 shows the envelope of the primary wave used in the controlled transition case studied in figure 4.18. It also shows the envelope of the wave packet along the centerline at a region where the primary wave in the control case reaches a maximum. The bottom figure shows results for the nonlinear packet. As shows previously, the primary waves are virtually unaffected by nonlinearity, the linear results shown are a representation of the 
linear part contained in the nonlinear packet. The results show that the amplitude of the primary wave in the controlled transition case is about $80 \%$ of the maximum amplitude in the packet. In other words it can be said that the effective amplitude of the packet in what concerns the subharmonic secondary instability is a little smaller than the maximum amplitude in the packet. The concept of effective amplitude was presented by (PAULA et al., 2013) in the context of streamwise-modulated-only waves, and also used by (MEDEIROS et al., 2015) in the context of spanwise-modulate-only waves. In both cases, the effective amplitude was the maximum amplitude in the packet. For modulation in both directions, the amplitude is a little lower.
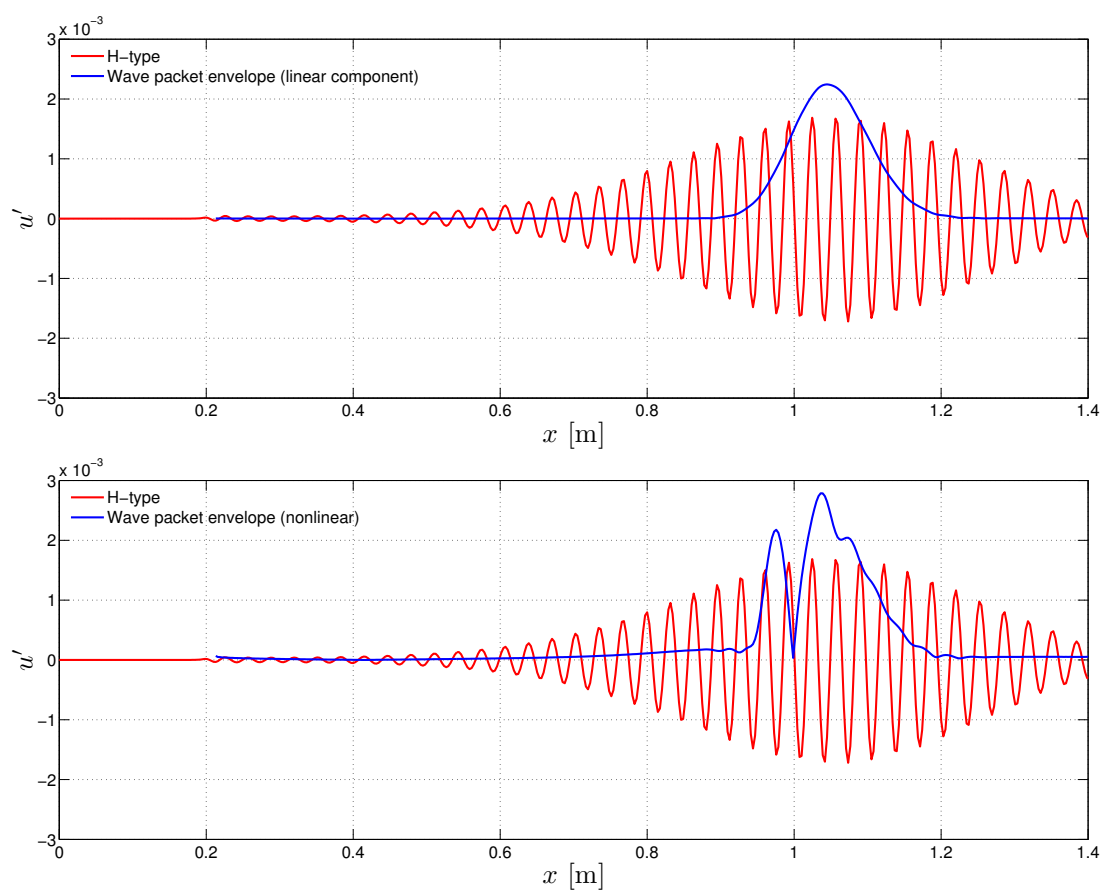

Figure 4.19: Analysis of the effective amplitude of the 2D wave driving the H-type instability in the packet. (a)Scaled linear packet. (b) Nonlinear packet. 
To study the evolution of the subharmonic band in more detail, the dominant subharmonic mode was identified at several nonlinear downstream positions, between $x^{*}=1000 \mathrm{~mm}$ and $x^{*}=1300 \mathrm{~mm}$. In figure 4.20 these modes are indicated on the spectrum at $x^{*}=$ $1300 \mathrm{~mm}$ with the colored circles. The most amplified subharmonic mode in the spectra reduces slowly its frequency and spanwise wave number downstream, probably up to reach a preferred mode, as (YEO et al., 2010) suggest. The corresponding amplification curves are plotted in figure 4.21, they are compared with the amplification curves for the oblique mode of the linear wave packet and the curves generated by the interaction of the triad of waves that induces H-type resonance for the same modes.

At $x^{*}=1000 \mathrm{~mm}$ the nonlinear growth rate of the oblique mode in the packet is driven by the corresponding 2D mode, as indicated by the threshold points (gray dashed lines). Its nonlinear amplification along the entire domain can be reproduced very closely with the controlled transition, including the location of the threshold amplitude. This behavior is similar for the neighbor modes, as can be seen in figure 4.22 .

In downstream positions small, but increasing deviations are observed from the controlled case, in the neighbor modes also are observed this kind of deviations. At $x^{*}=1300 \mathrm{~mm}$ the nonlinear amplification curve can not be explained by the triad interaction for the controlled correspondent case. As conclusion, nonlinerities present in the wave packet evolution that can not be generated by the three-wave interaction are weak in the first half of the domain, but increase in downstream and become comparable to the subharmonic growth, finally dominates the nonlinear amplification rate of the subharmonic band.

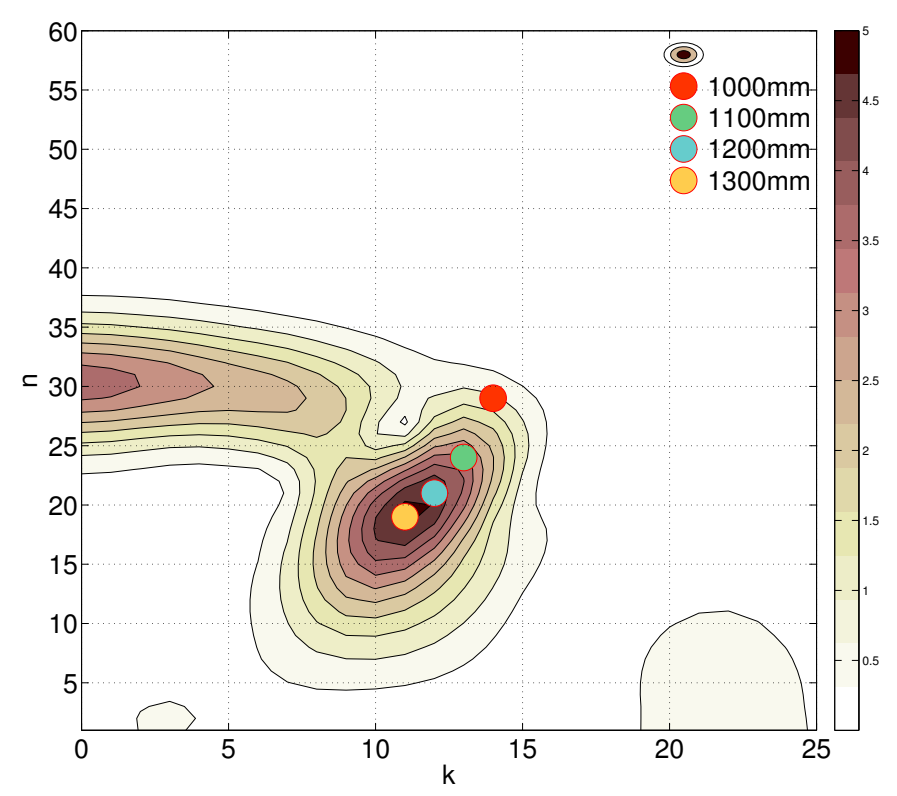

Figure 4.20: Subharmonic most amplified mode at several streamwise positions between $x^{*}=1000 \mathrm{~mm}$ and $x^{*}=1300 \mathrm{~mm}$. 
(a)
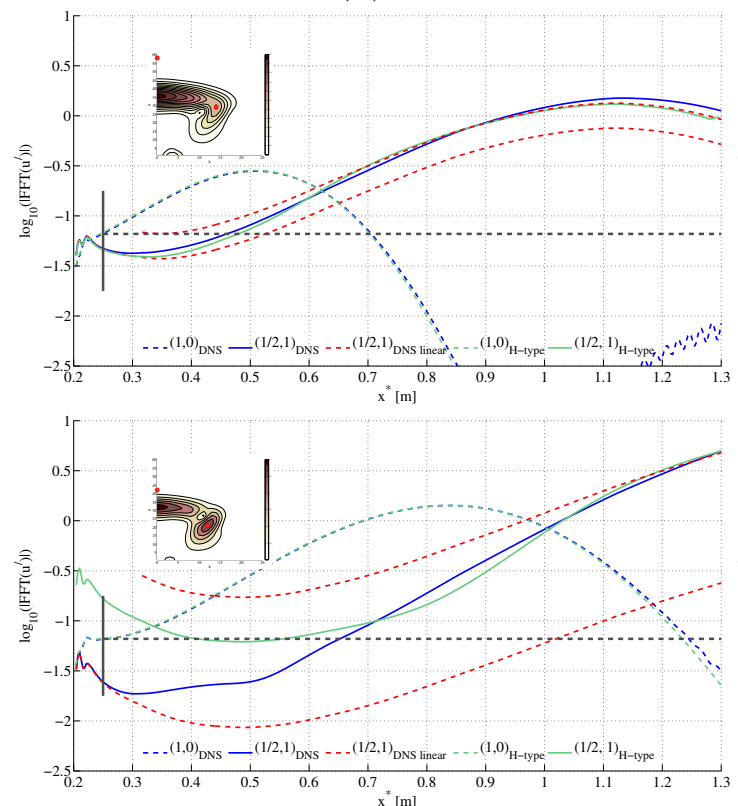

(c) (b)
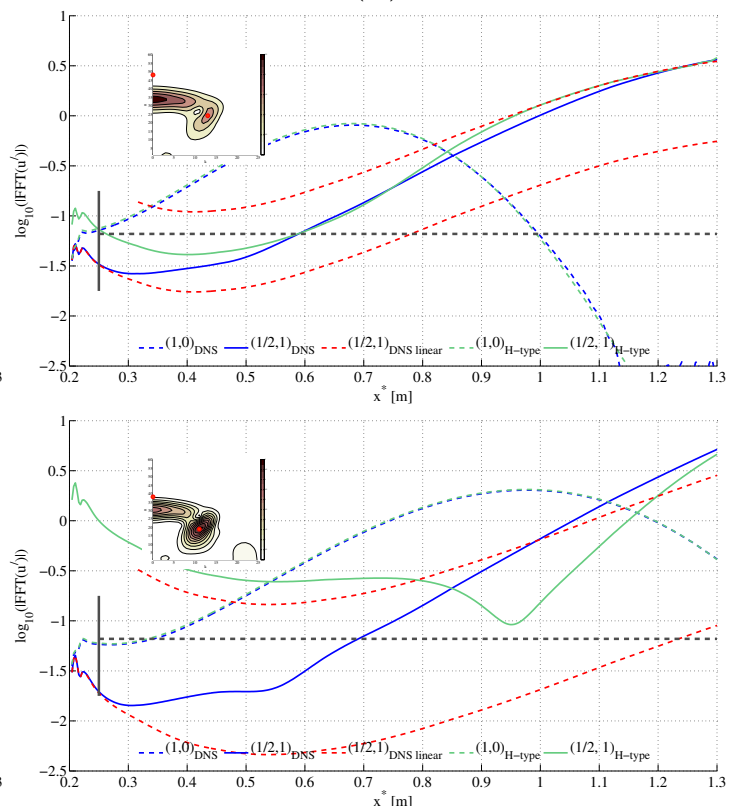

(d)

Figure 4.21: Amplification curves for the most amplified subharmonic modes denotes as $(\mathrm{n}, \mathrm{k})$. $(19,11)_{x=1300 \mathrm{~mm}}$.
(a) $(29,14)_{x=1000 \mathrm{~mm}}$
(b) $(24,13)_{x=1100 m m}$,
(c) $(21,12)_{x=1200 \mathrm{~mm}}$,
(d)

(a)

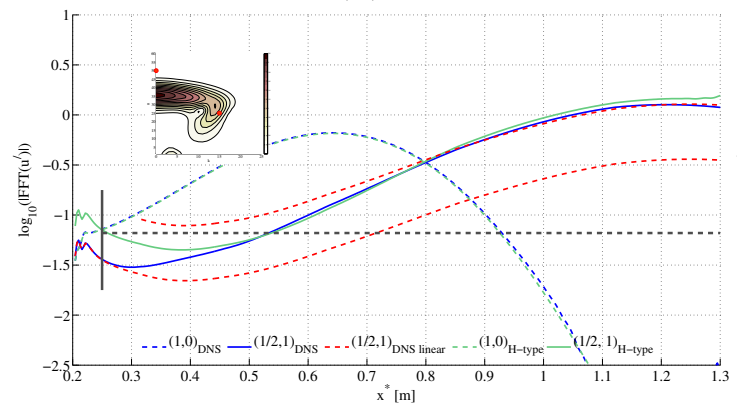

(b)

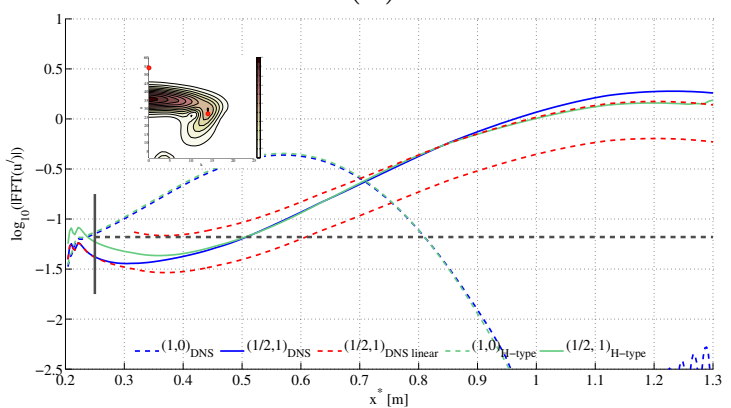

Figure 4.22: The nonlinear amplification of subharmonic modes near to the peak in the subharmonic band, at $x^{*}=1000 \mathrm{~mm}$, can be reproduced closely with H-type controlled transition. (a) 25, 15, (b) 27, 14 . 


\subsubsection{Asymmetry generated by the nonlinear effects}

From the amplification curves it is clear that subharmonic modes grow nonlinearly from a position downstream near the disturbance source. However in the time signal velocity their effect is not visible due to subharmonic low levels. When the subharmonic component in the packet is in phase with its correspondent fundamental mode (2D), their amplification is optimized, and growing deformation of the wave packet is observed in downstream, as can be seen in figure 4.23. This is caused by different propagation velocities inside the wave packet, as is indicated by the dotted lines and the points in figure 4.23.

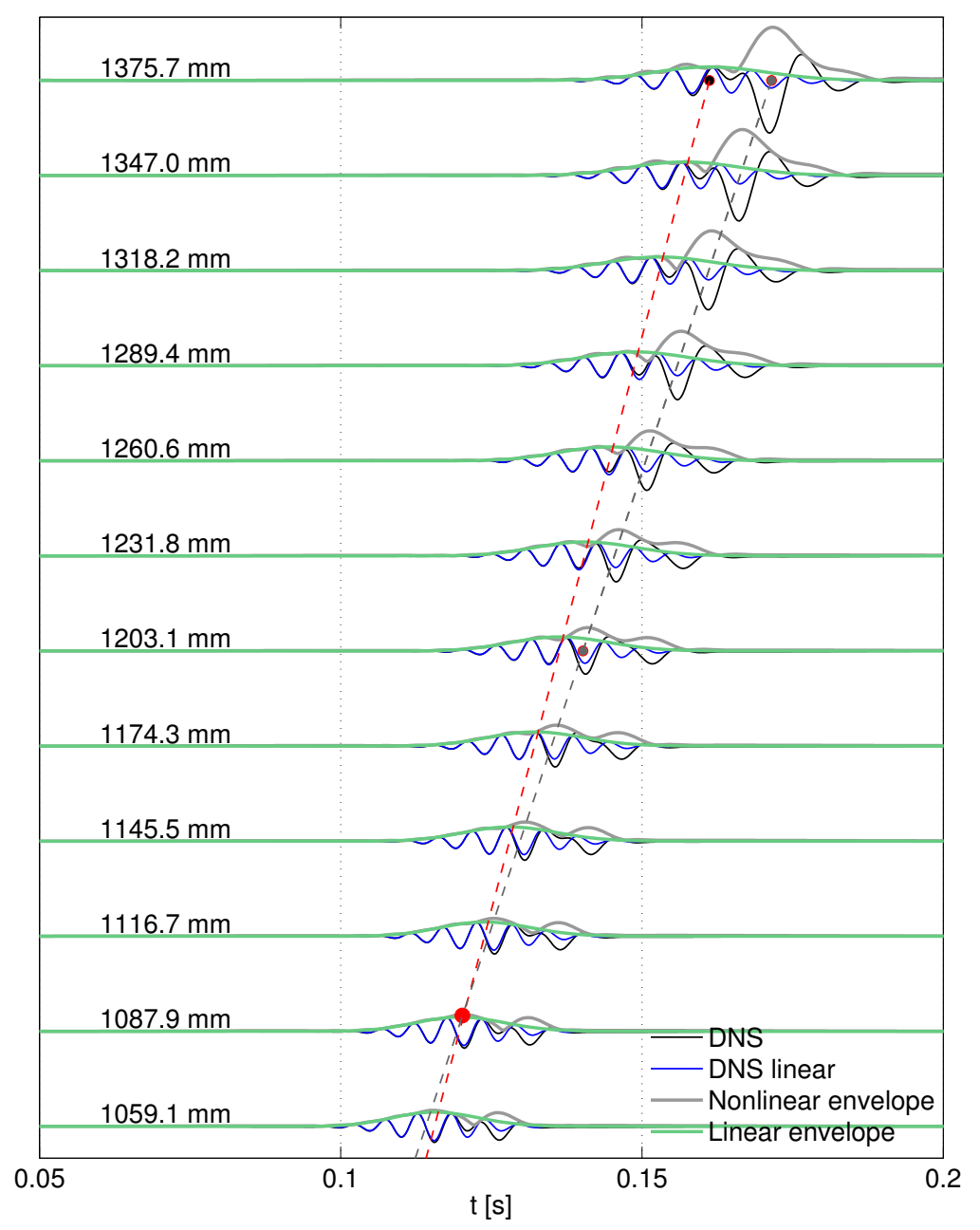

Figure 4.23: Effect of subharmonic nonlinearity on wave packet shape.

\subsubsection{Subharmonic seed}

Following (MEDEIROS; GASTER, 1999b), to investigate the origin of subharmonic modes, the subharmonic band was removed from the perturbation spectrum (figure 4.24). In the resulting spectra (figure 4.26), subharmonic modes develop almost equal to the previous scenario (see figures 4.18 and 4.25), with the complete perturbation spectrum. Then, subharmonic modes are generated by nonlinear interaction, as is evident from the am- 
plification curves (see figure 4.26)), however the responsible mechanism is unknown, but there are some tentative explanations (CRAIK, 2001).

To evidence the generation and amplification of the subharmonic band, in the figure 4.27 are plotted the amplitude curves for the mode $(24,13)$ (indicated by the black lines), which corresponds to the most amplified subharmonic mode at the position $x^{*}=110 \mathrm{~mm}$. For this mode, the subharmonic generation process, develops from the perturbation to the position in downstream around $x^{*}=0.6 \mathrm{~m}$, then, nonlinear amplification occurs as in the case with full spectrum content at the disturbance. In the packet, this kind of nonlinearity acts in conjunction with the $\mathrm{H}$-type resonance.

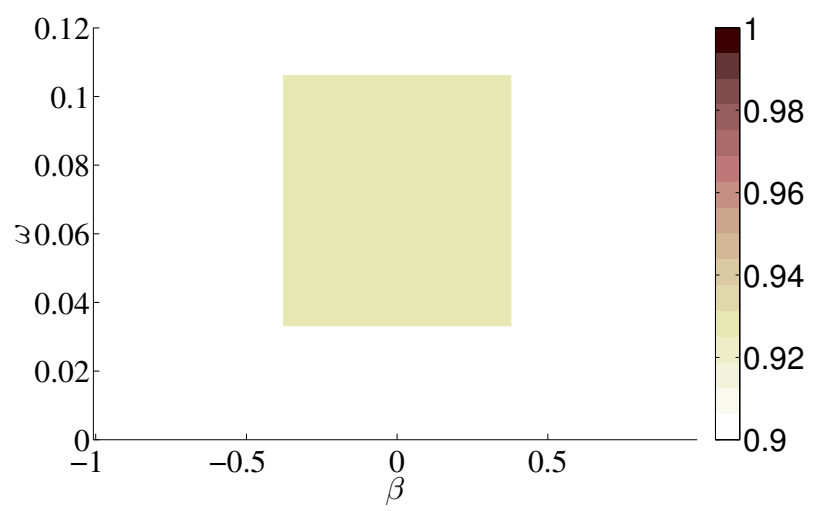

Figure 4.24: Spectrum of the perturbation with subharmonic band removed.
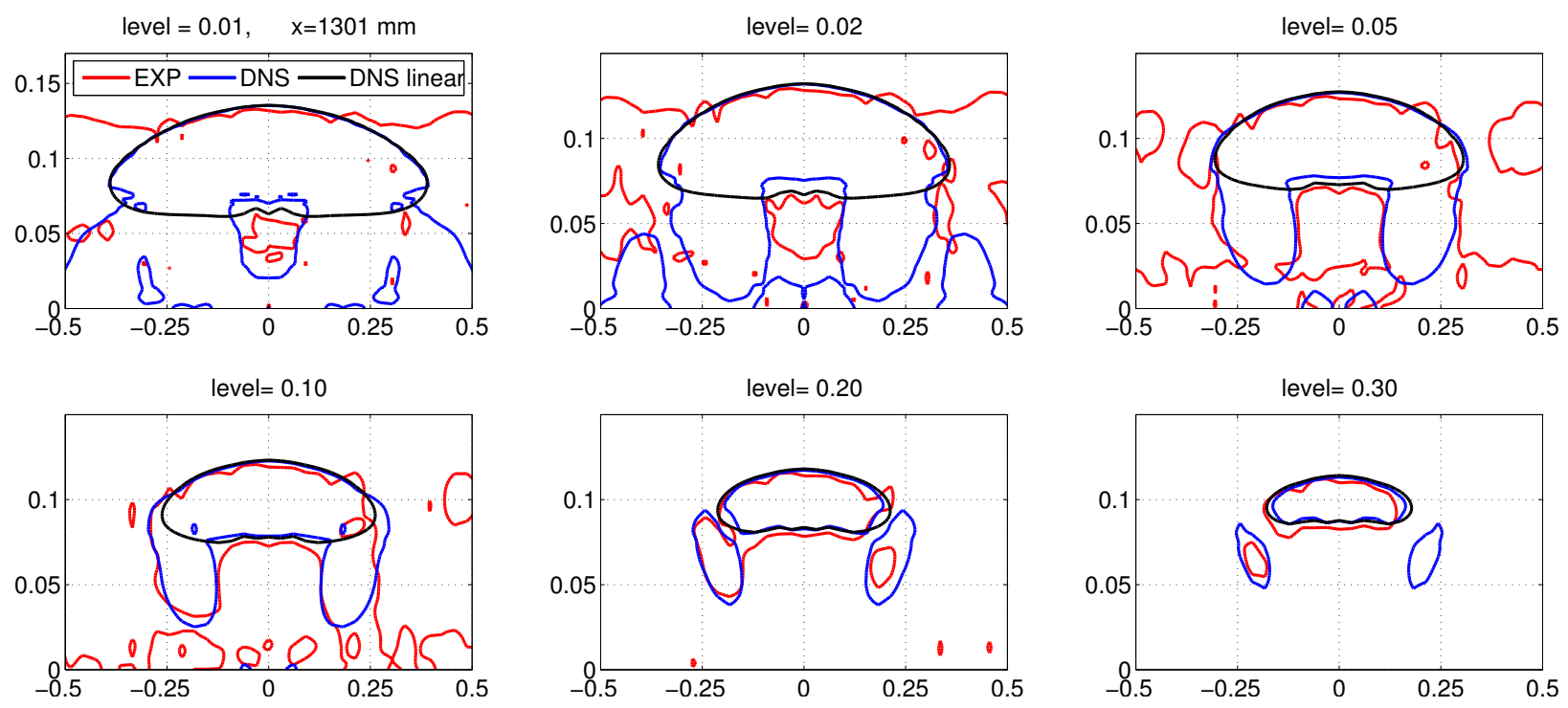

Figure 4.25: Comparison of spectrum levels at $\mathrm{x}=1300 \mathrm{~mm}$, to investigate subharmonic seeding. 

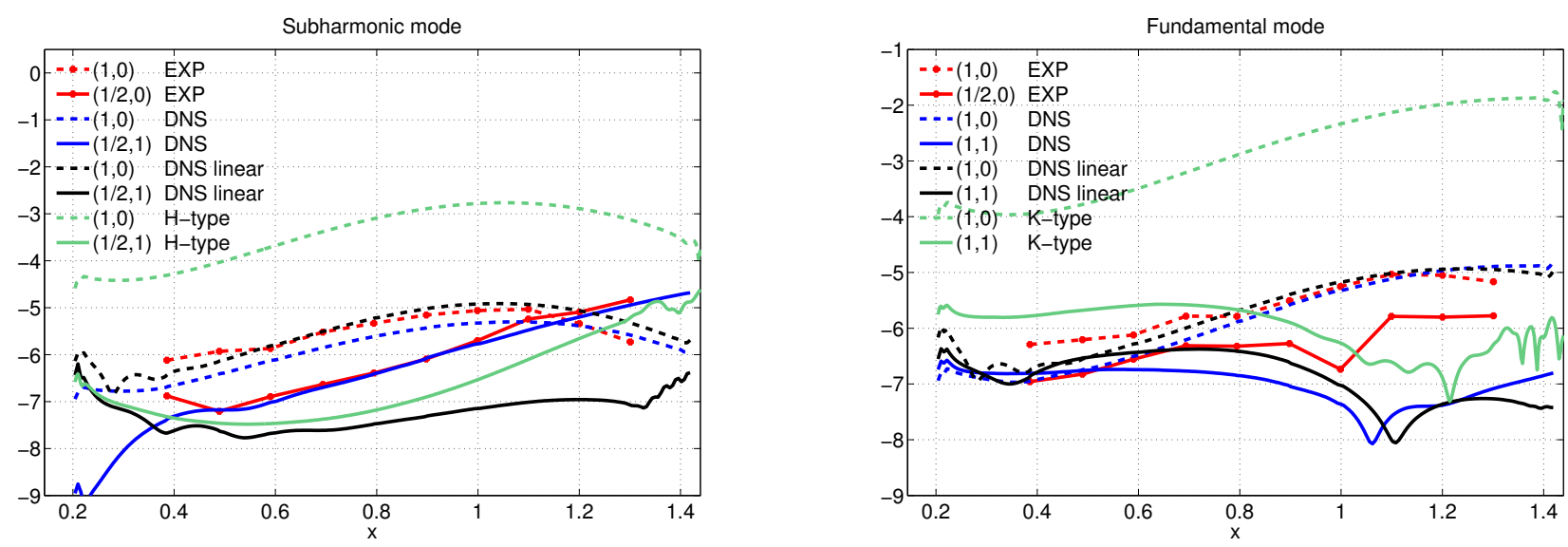

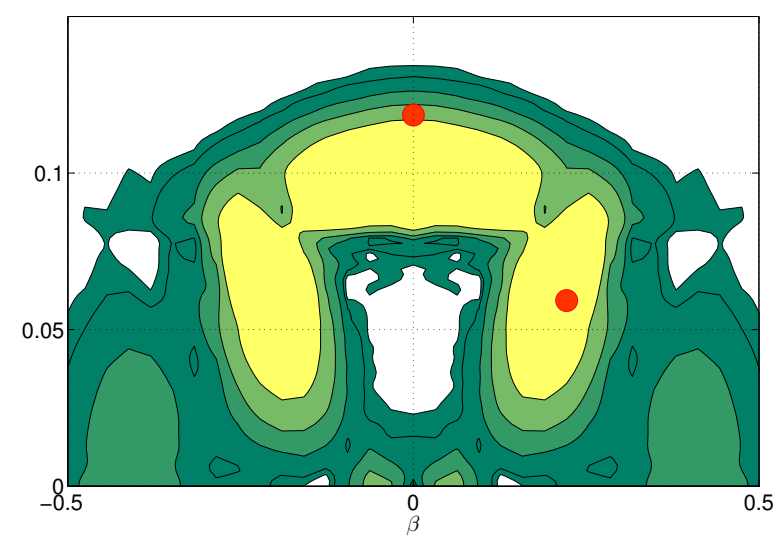

(a)

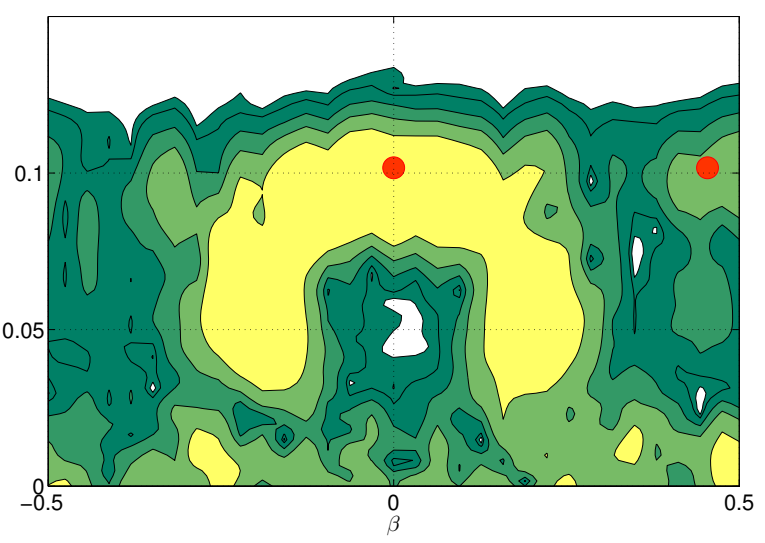

(b)

Figure 4.26: Amplification curves and spectrum of the wave packet generated with subharmonic band removed from the perturbation spectra.

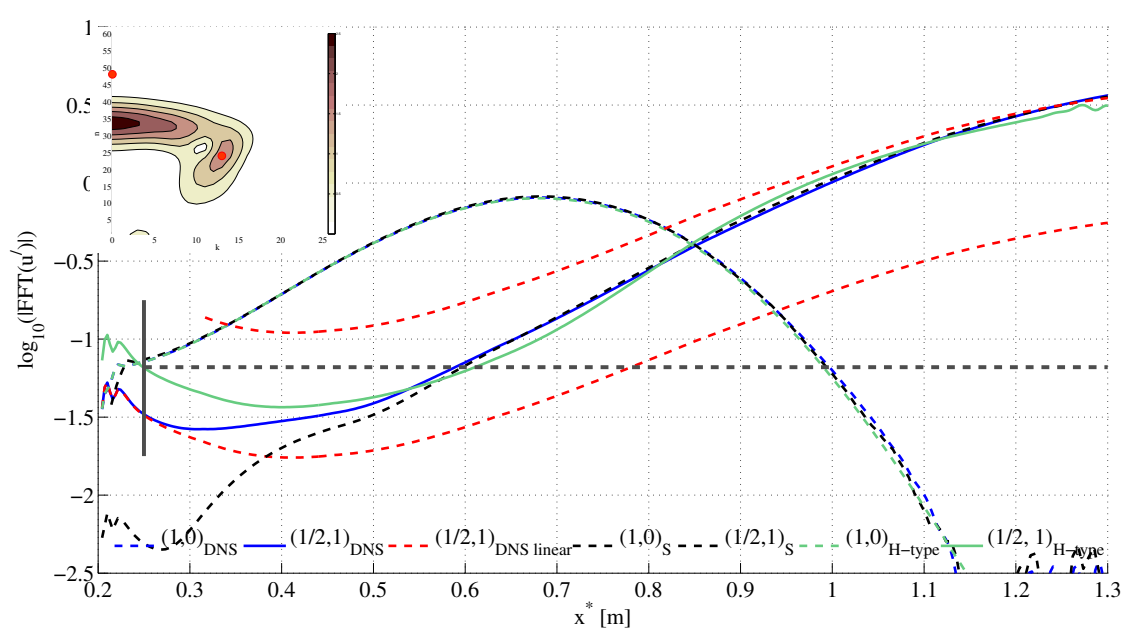

Figure 4.27: Amplification curve of the mode $(24,13)$ of the subharmonic mode generated in the reduced disturbance spectrum. 


\subsubsection{Fundamental bands}

In the fundamental band two group of modes were selected by changing their frequencies, they are indicated by the yellow circles in figure 4.28. The first band, shown in the top frames, has the same frequency of the dominant 2D mode, that could amplify as $\mathrm{K}$ type breakdown. The second band, shown in the bottom frames, has higher frequencies, maintaining the same spanwise wave numbers of the first band. The modes are identified by their indices $(n, k)$ in the spectrum. These bands are studied because (COHEN, 1994) shows strong nonlinear amplification in these bands, but without look for a cause. Also, (MEDEIROS, 2004) report similar amplification for lower amplitudes of the wave packet . Amplification curves reveals that nonlinear growth dominates from downstream positions around $x^{*}=800 \mathrm{~mm}$ and $x^{*}=700 \mathrm{~mm}$ for the first and the second group respectively.

On each group a mode was selected, to verify fundamental resonance condition. In figures 4.29 and 4.30 locking between 2D fundamental wave and the oblique mode can be observed, for both bands. In the second band, locking occurs in a upstream position than in the first band, because the 2D wave reach threshold amplitude before, then, the nonlinear amplification is triggered early for higher frequencies (see figure 4.11).
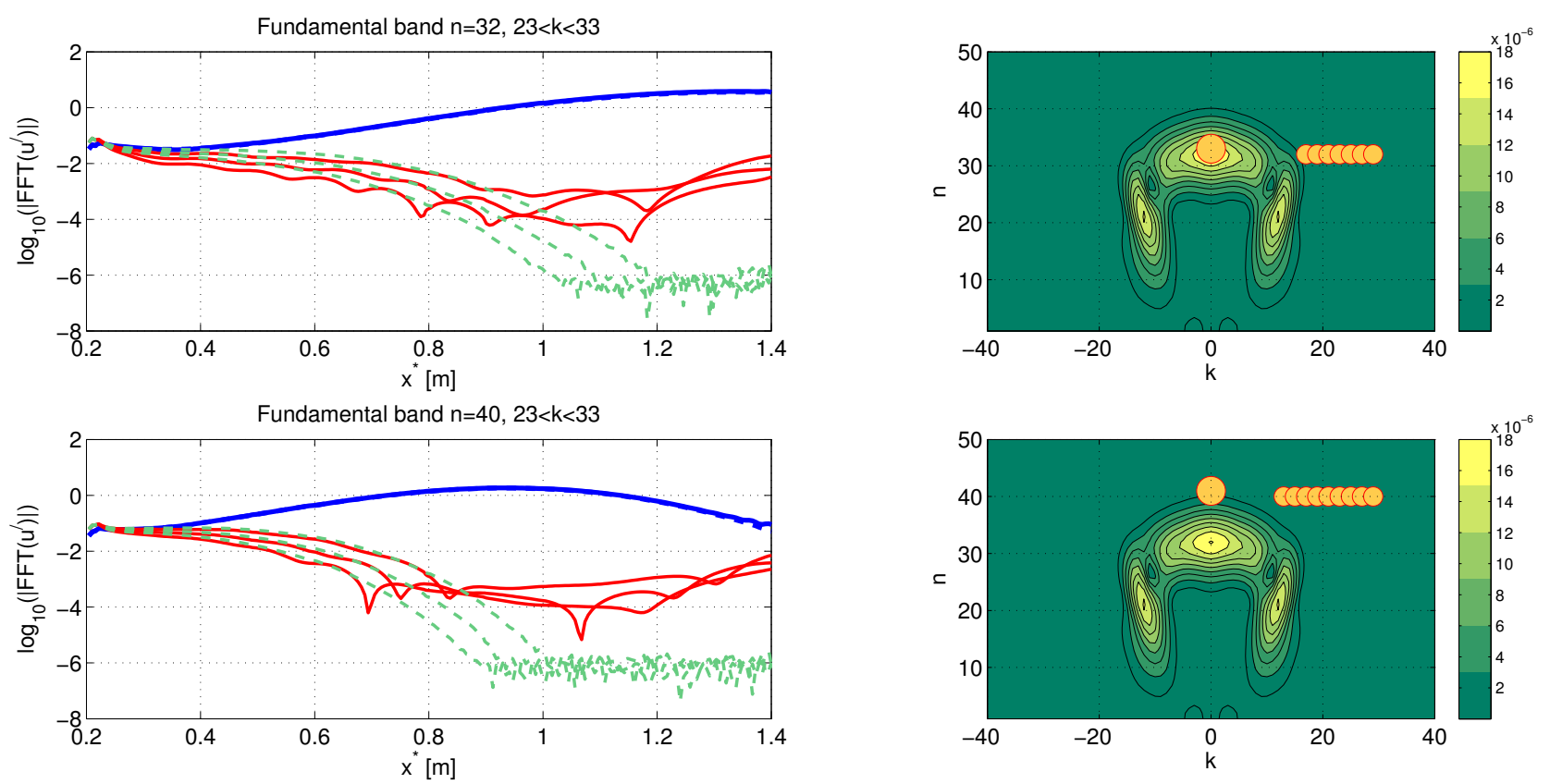

Figure 4.28: Amplification curves for fundamental bands of linear (green lines), nonlinear (red lines) and fundamental (blue lines) modes. 

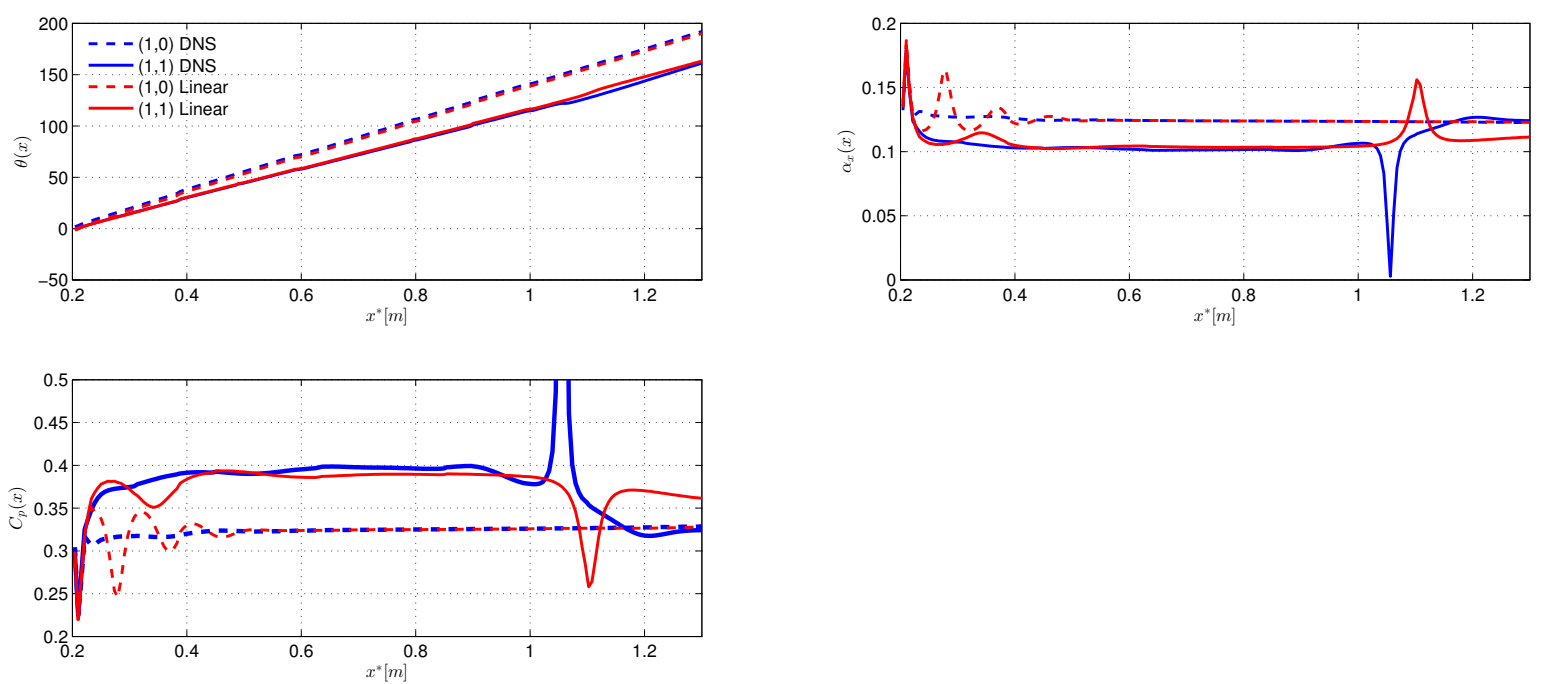

Figure 4.29: Mode of the first band, $n=32, k=25$, (a) Phase, (b) streamwise wave number, (c) locking in phase velocity for fundamental resonance.
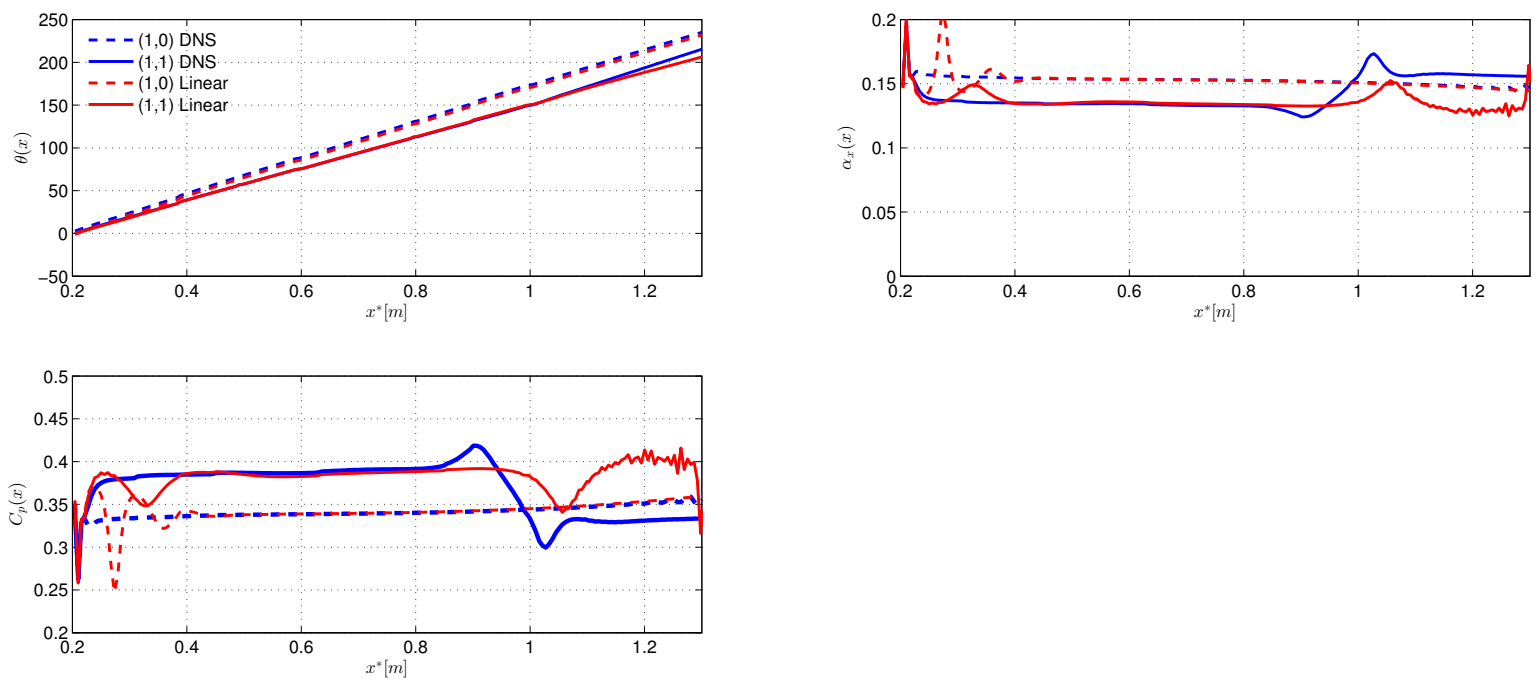

Figure 4.30: Mode of the second band 2, $n=40, k=25$, (a) Phase, (b) streamwise wave number, (c) locking in phase velocity for fundamental resonance.

From the experimental spectrum it was chosen the most amplified in the fundamental band, with its corresponding 2D mode, as indicated in the figure 4.31(b) by the red circles. For the 2D and oblique mode, the amplification curves (figure 4.31(a)) are compared for experimental, simulation and DNS linear reference case. Also, a controlled case of K-type resonance was generated to reproduce the nonlinear growth rate for the oblique mode. The resultant amplifications curves are plotted in green lines. The growth rate calculated in this manner is very similar to that observed in the packet. However the level at the last positions is underestimated in the DNS simulations. In following sections this fact is considered in detail. The effective amplitude (figure 4.32) is around $20 \%$ larger than the maximum wave packet amplitude. 

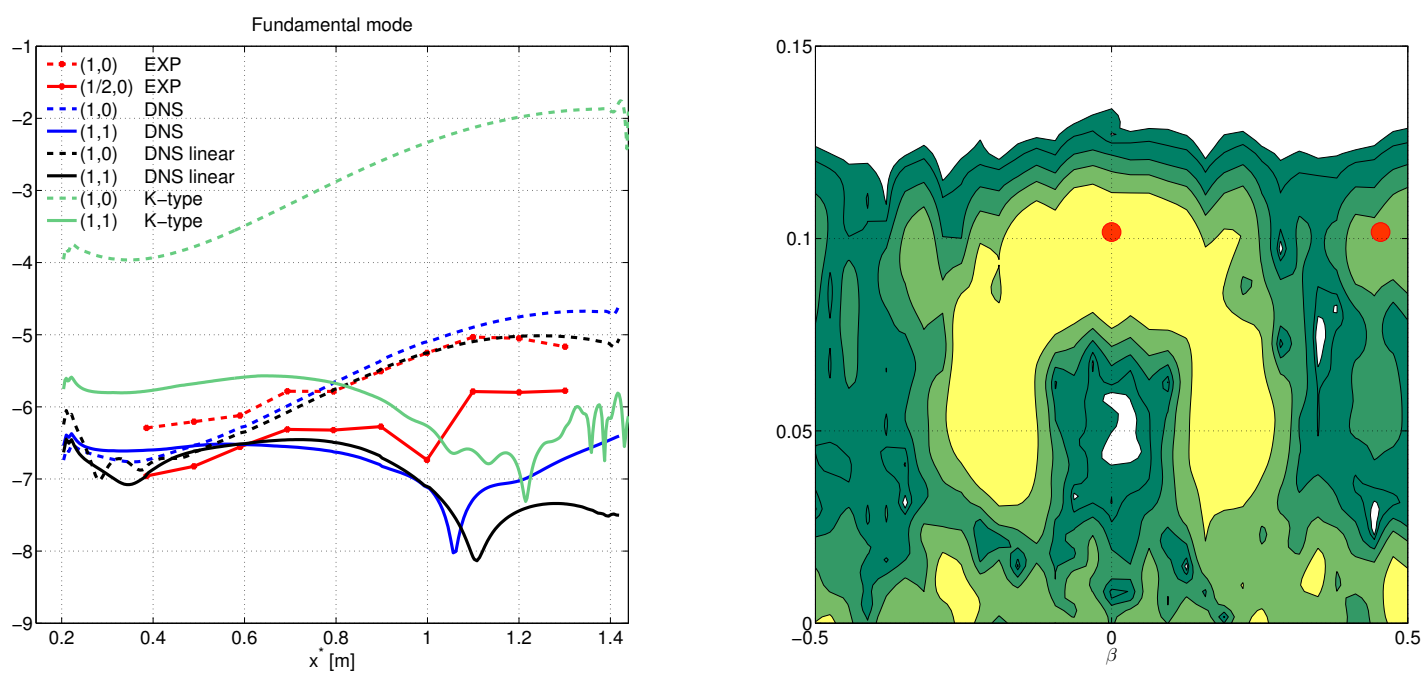

Figure 4.31: Amplification curves of the most amplified mode in the fundamental band of the experimental spectrum.

\subsubsection{Effective amplitude for fundamental resonance}

To reproduce nonlinear growth rates for modes oblique modes $(1,1)$ by controlled transition of K-type breakdown, the threshold amplitude of the 2D wave must be higher than the wave packet amplitude. In the experiment and simulations, this kind of instability rises in the last measurement points with higher growth rates than H-type resonance.
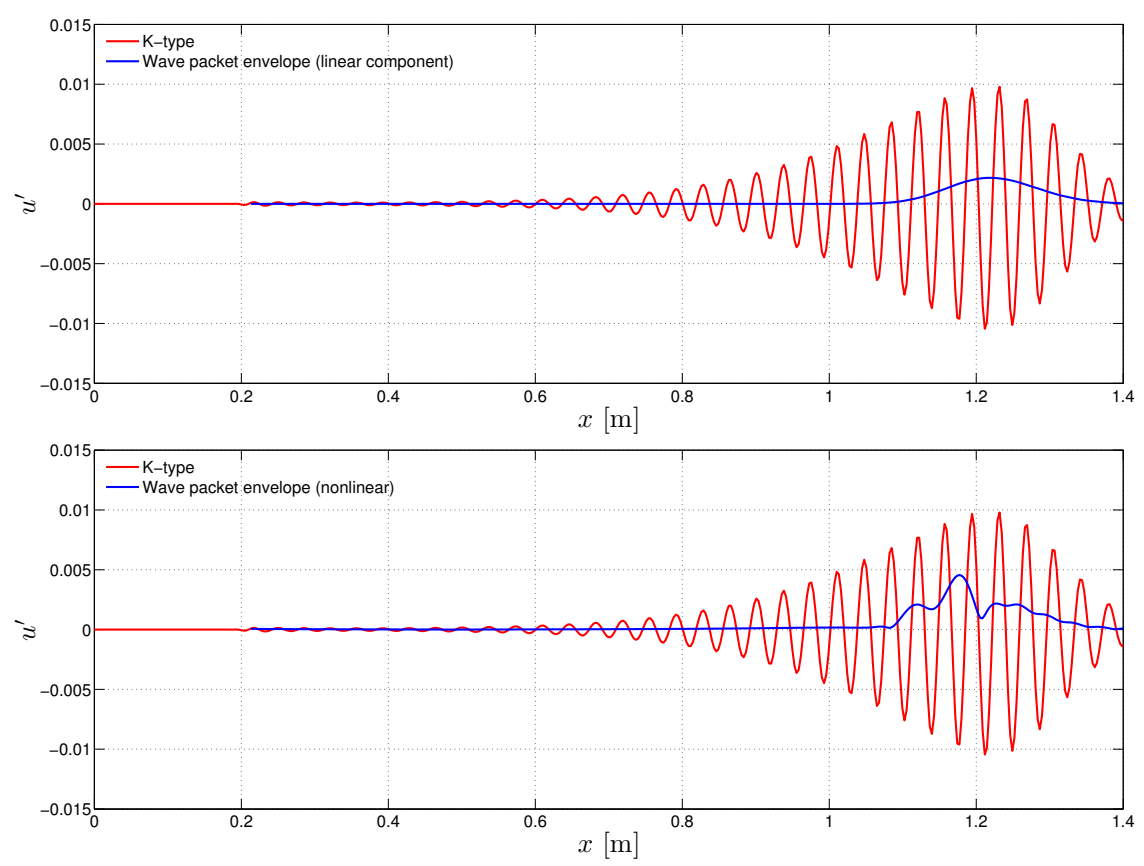

Figure 4.32: Effective amplitude for K-type breakdown. 


\subsection{Effective experimental conditions}

In previous sections, it was shown that main difference between DNS and experimental results was in the modes associated with K-type breakdown. Nonlinear growth rates for low frequency modes and fundamental ones, calculated from DNS are in accordance with experimental growth rates, however, these modes have lower levels in DNS than observed experimentally. To attempt identify the cause of the levels mismatch in the experiment, some effective experimental imperfections were investigated.

\subsubsection{Pressure gradient}

The pressure coefficient $C_{p}$ shown in figure 4.33 corresponding to the measured in the experiment, was included into pressure distribution at the outerflow boundary. Amplification curves were obtained for a wave packet with the experimental pressure gradient, for a subharmonic (figure 4.34(a) ) and a fundamental mode (figure 4.34(b)). From the amplitude curves it can be concluded that the experimental pressure gradient has no effect on the fundamental modes level, because the difference in levels of the mode $(1,1)$ between DNS result and experiment remains almost equal as in the previous case.

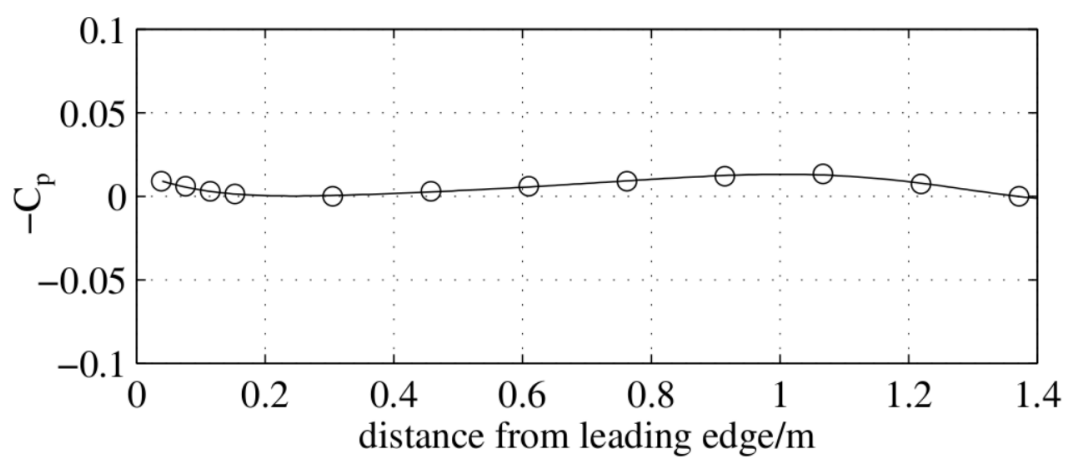

Figure 4.33: Experimental pressure gradient curve. (Reproduced from (MEDEIROS; GASTER, 1999b).) 

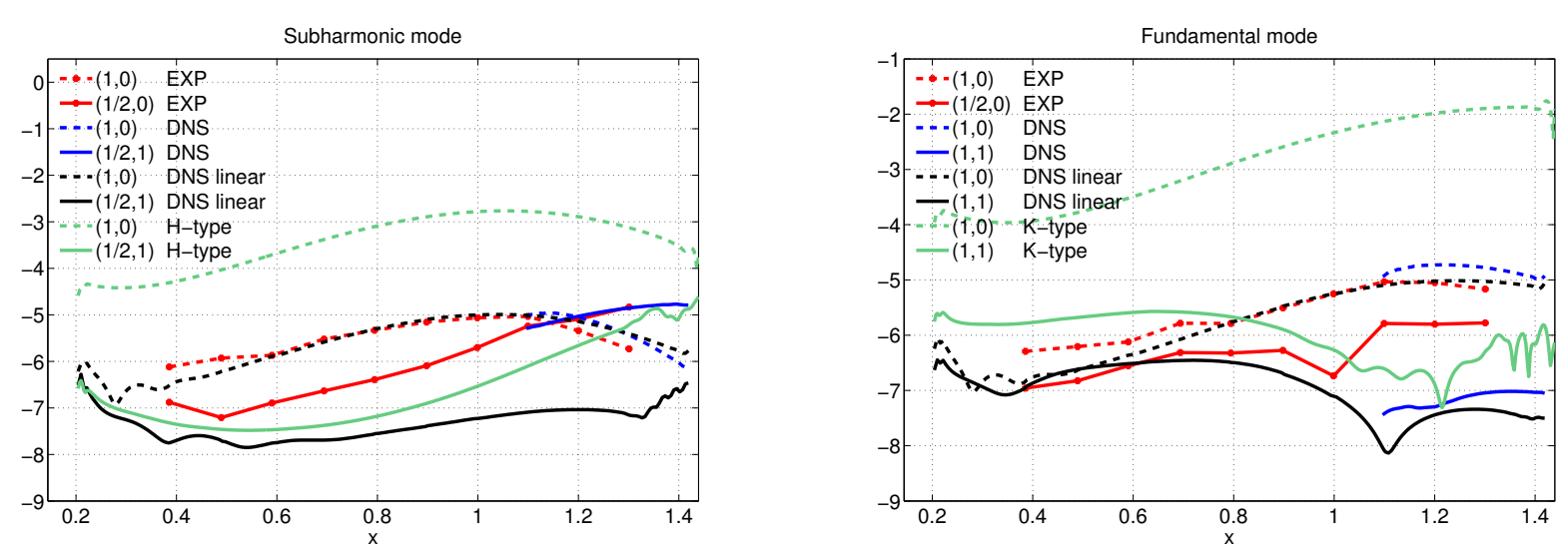

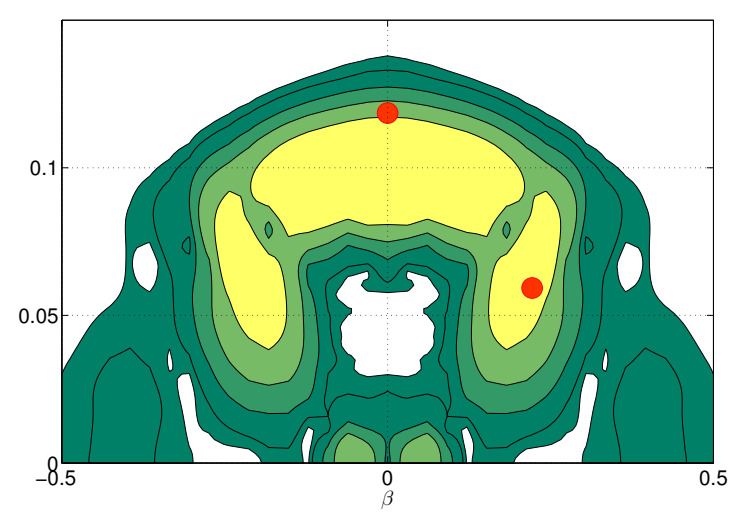

(a)

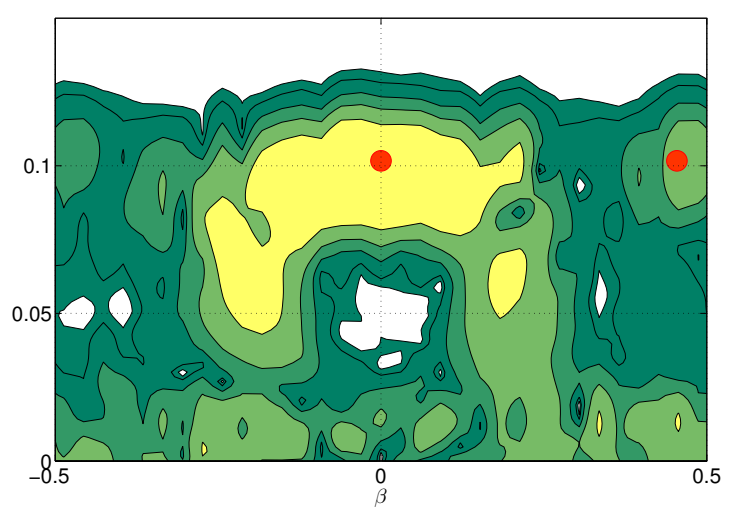

(b)

Figure 4.34: Amplification curves and spectrum at $x^{*}=1200 \mathrm{~mm}$, for a wave packet with the experimental pressure gradient, (a) subharmonic and (b) fundamental mode.

\subsubsection{Amplitude calibration}

The experiment displayed a asymmetry in spanwise direction, which was not present in DNS simulations due to the idealization of the problem. To evaluate the effect of this factor in the fundamental mode amplification, a modal calibration in Fourier space was done, in the linear region. This strategy was used successfully in (MAYER; WERNZ; FASEL, 2011) to compare numerical and experimental data. It is based on weighting each mode, to have the same amplitude of the experimental spectrum in a linear position.

Calibration was done at $x^{*}=600 \mathrm{~mm}$. Also the experimental pressure gradient was considered. In the spectrum at position $x^{*}=1300 \mathrm{~mm}$, from calibrated spectrum (figure 4.35) can be observed that fundamental modes remains underestimated. Moreover the subharmonic bands present a higher deviation from experimental, than obtained for the simulation with uniform spectra.

Also (BREUER; COHEN; HARITONIDIS, 1997) shows that spectrum of the ensemble-averaged is different from the ensemble average of the individual spectra. It is not clear DNS result 
to which can be associated. For the present analysis results were compared directly, but in future works this detail deserve more attention.
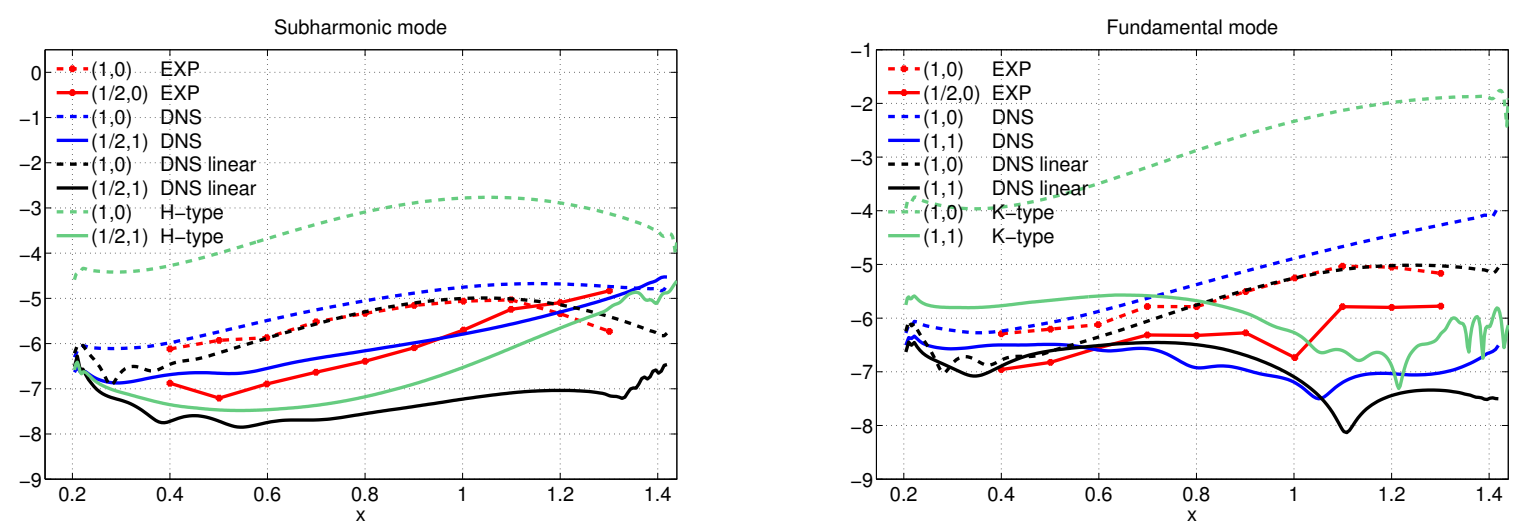

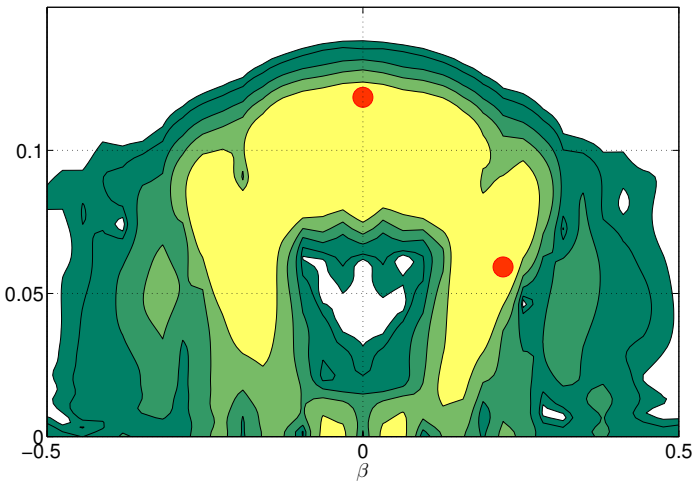

(a)

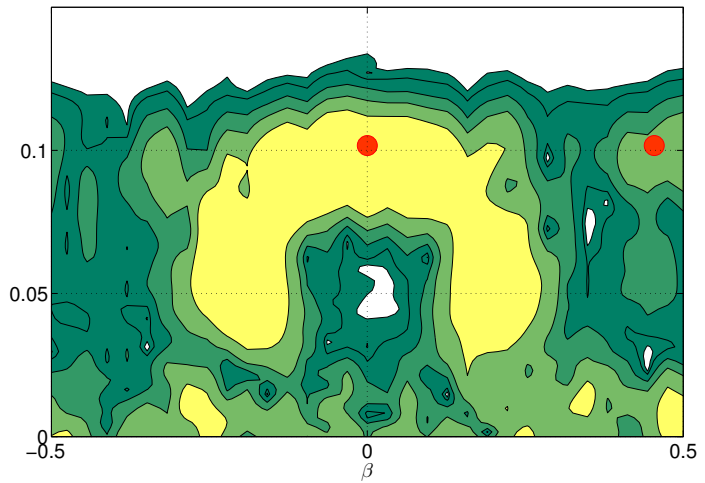

(b)

Figure 4.35: Amplification curves and spectrum at $x^{*}=1200 \mathrm{~mm}$, for wave packet with experimental pressure gradient and spectrum calibration at position $x=600 \mathrm{~mm}$. (a) Subharmonic and (b) fundamental mode.

\subsubsection{Amplitude effect}

Experimental (OPFER, 2002) and numerical works (SPALART; YANG, 1987) relate the high levels of fundamental modes with disturbance amplitude. With similar flow parameters, (OPFER, 2002) establishes a linear correlation between the level of the modes in the fundamental band and the disturbance amplitude. Furthermore, notes that the spanwise wave numbers of these modes are around twice of the highest linearly unstable oblique modes (see figure 4.36). 


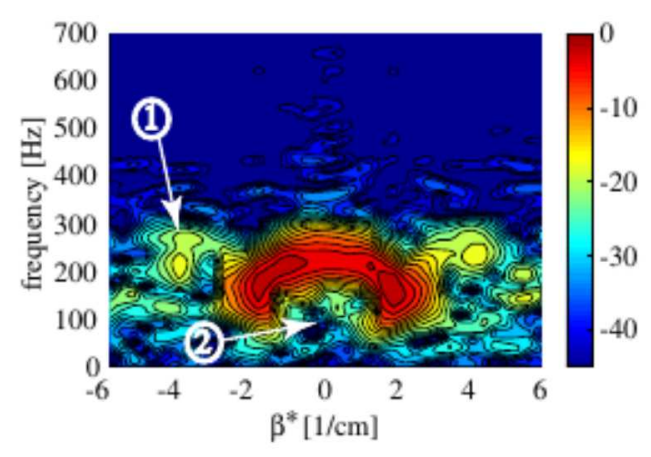

(k) $x=70 \mathrm{~cm}, R e=913$

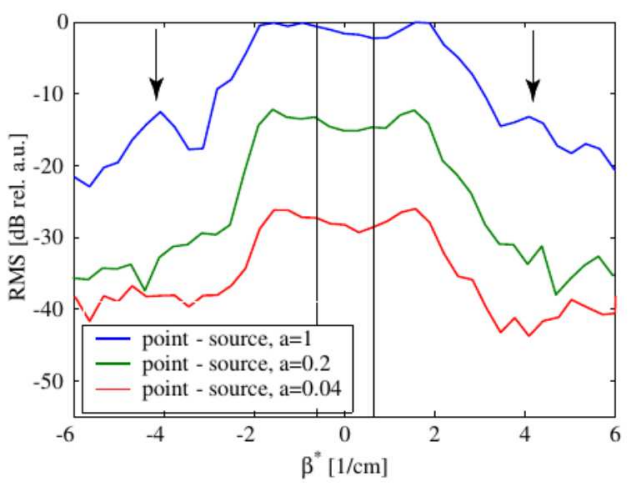

Figure 4.36: 1) Higher levels of the disturbance amplitude are linearly related to higher levels of the fundamental modes. Reproduced from (OPFER, 2002, figures 7.10 and 7.11).

To investigate the amplitude effect, a wave packet generated with an amplitude \%20 higher than in previous case was simulated. In figure 4.37 it can be seen that the resulting spectra has levels in fundamental band that are similar to the experimental ones, however, low frequency modes present higher amplification. Also, the subharmonic modes are overestimated.
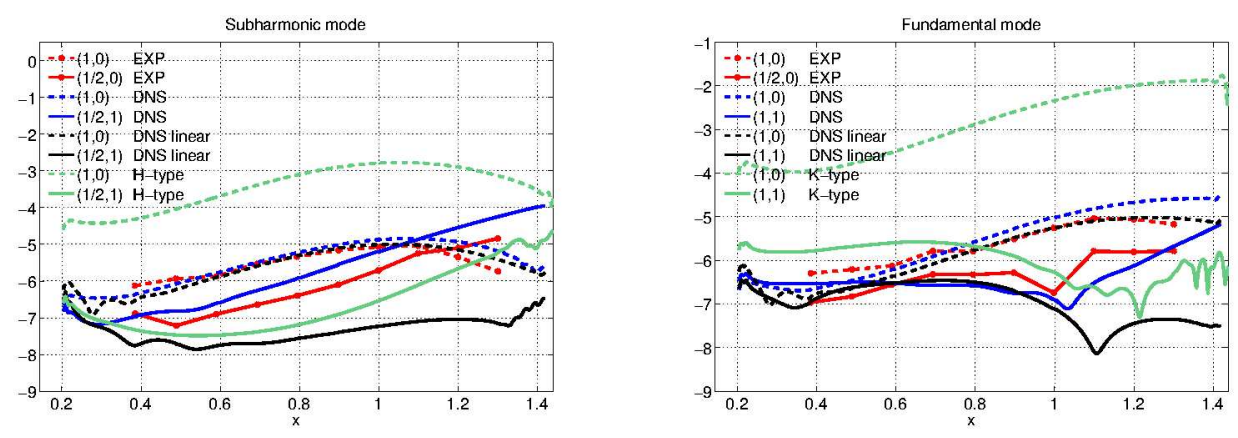

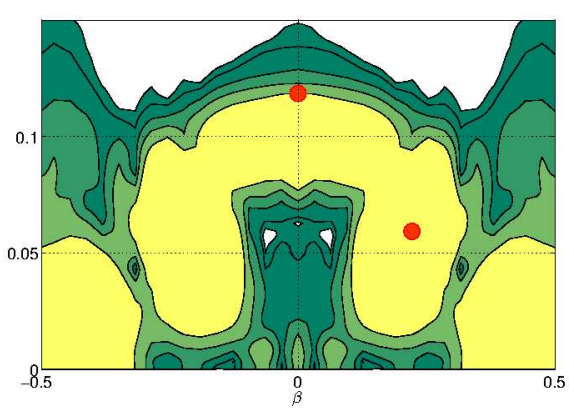

(a)

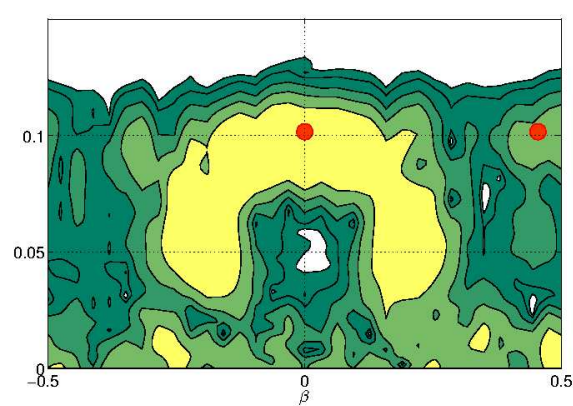

(b)

Figure 4.37: Amplification curves and spectrum for a wave packet with disturbance amplitude increased 20\%. (a) subharmonic and (b) fundamental mode.

From the spectrum in figure 4.37 , the fundamental resonance seems to be concentrated 
around the band centered in $\omega=0.09075$. In the figure 4.38, the amplitude of the corresponding spanwise modes is plotted. Considering as fundamental modes, the modes centered in $(0.0975,0.125)$, denoted as $(1,1)$. It is observed a nonlinear amplification in the spanwise harmonics, $(1,2 \beta)$ and $(1,3 \beta)$, which suggest the presence of K-type mechanism in this band.

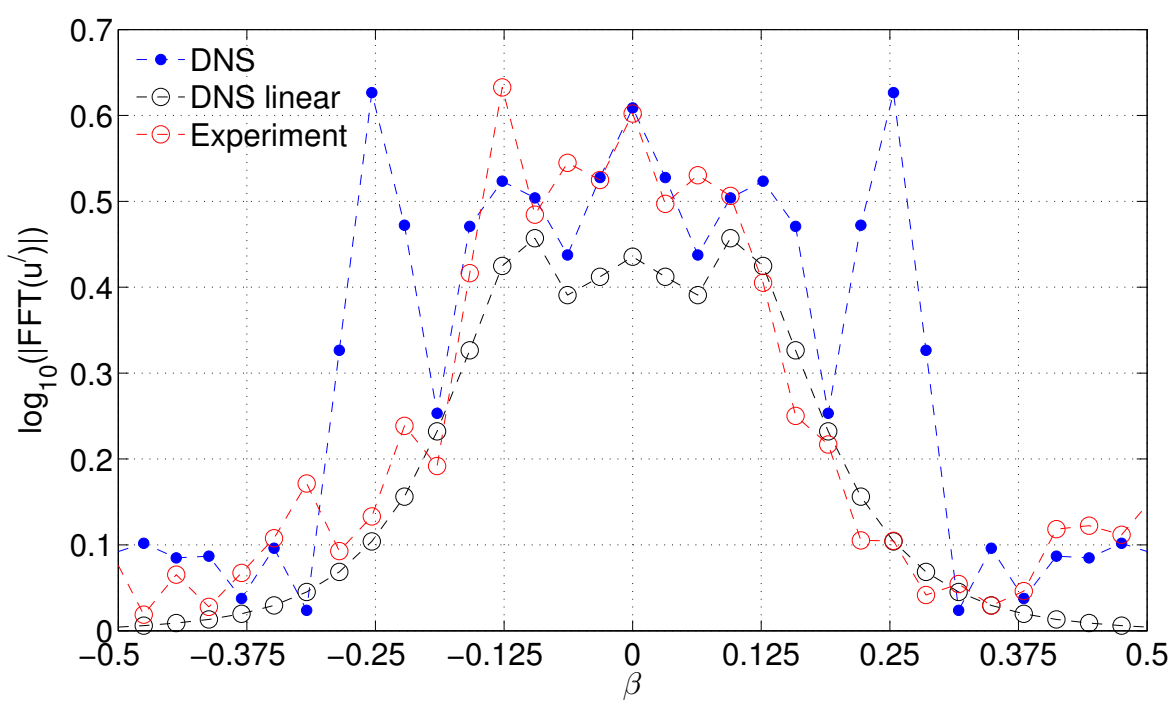

Figure 4.38: Spanwise modes for $\omega=0.09075$ at $x=1300 \mathrm{~mm}$. 


\section{Wave packet in compressible boundary}

layer

Some observations at Mach 0.5, considering white noise (NORMAND; LESIEUR, 1992), nonlinear growth (WASISTHO; GEURTS; KUERTEN, 1997), development of transition in an airfoil (LIU et al., 1997) and secondary instability mechanisms (DENG et al., 2005) suggest that up to Mach 0.5 compressibility has a weak influence on the initial nonlinear stages of transition in the boundary layer. Then, for the present analysis boundary layers at Mach $>0.5$ were considered.

This section is organized as follows. Initially the numerical set-up is defined. Next, the linear and nonlinear evolution of a wave packet is investigated by DNS simulation, considering a boundary layer on a flat plate at Mach 0.7 and 0.9. The main differences for the wave packet in an incompressible boundary layer are established and the early nonlinear stage is investigated. Finally, to consider a scenario that may be closer to natural transition, results for interactions of two wave packets at Mach 0.9 are presented.

\subsection{Numerical set-up}

To reduce computational time required to perform the simulations, it was considered as reference case the experimental parameters of (COHEN; BREUER; HARITONIDIS, 1991), for incompressible boundary layer. This experiment introduces a relative high level of disturbances amplitude which induces development of transition in a smaller region of the boundary layer in streamwise. The disturbance function was the same considered for the incompressible case, covering the same bands and controlling the perturbation spectrum as shown in section 4.2. The perturbation was located at the position $x_{0}=200$. From the experimental parameters defined in the table 1 the computational domain is:

$0 \leq x \leq 1200$

$0 \leq y \leq 20 \approx 3.5 \times \delta_{99}(x=1200)$

$-\frac{\pi}{\beta_{0}} \leq z<\frac{\pi}{\beta_{0}}$ 
It was employed the same grid resolution as in the incompressible boundary layer, the resultant number of grid points in each direction is $n x=601, n y=51, n z=160$. Some tests, not shown here, reveals grid independence for this computational arrangement. The flow parameters for DNS simulation are $R e=1035, \operatorname{Pr}=0.71$, and the considered Mach numbers, $M_{\infty}=0.7$ and $M_{\infty}=0.9$. In all simulations isothermal wall was considered. with $T_{w}=1$.

\subsection{Wave packet in a boundary layer at Mach 0.7}

At Mach 0.7 three wave packets were simulated, a low amplitude packet (linear) to be used as reference case and two nonlinear packets with amplitudes $a_{1}=3.5 \times 10^{-5}$ and $a_{2}=2.5 \times 10^{-5}$. The perturbation amplitudes were chosen to guarantee a linear region for at least a short distance from the perturbation location. This condition is verified by comparison of the amplification curves for several modes with the linear case.

\subsubsection{Linear wave packet}

The N-factor allows to identify the most linearly unstable mode at a fixed spanwise wave number. The N-factor is defined as:

$$
N=\ln \left\{\frac{A(x)}{A_{\text {ref }}}\right\}
$$

As illustration, for Mach 0.7, the resultant N-factor curves are shown in figure 5.1. The most unstable mode has the highest value of $\mathrm{N}$, in the figure, it is indicated by the red line. From the current DNS simulations for linear wave packets at several Mach numbers, the most unstable 2D and 3D mode were identified from the N-factor curves and spectrum. Results, shown in figure 5.2, reveals that for Mach $\leq 0.7$ the most amplified mode is $2 \mathrm{D}$, indicated by $(1,0)$. At higher Mach numbers, a band of oblique modes centered in $\beta \sim 0.07$ are linearly more unstable. In all cases, the frequency of these modes decays with the Mach number. As a consequence, the modes influenced by the nonlinear amplification may change with the Mach number. 


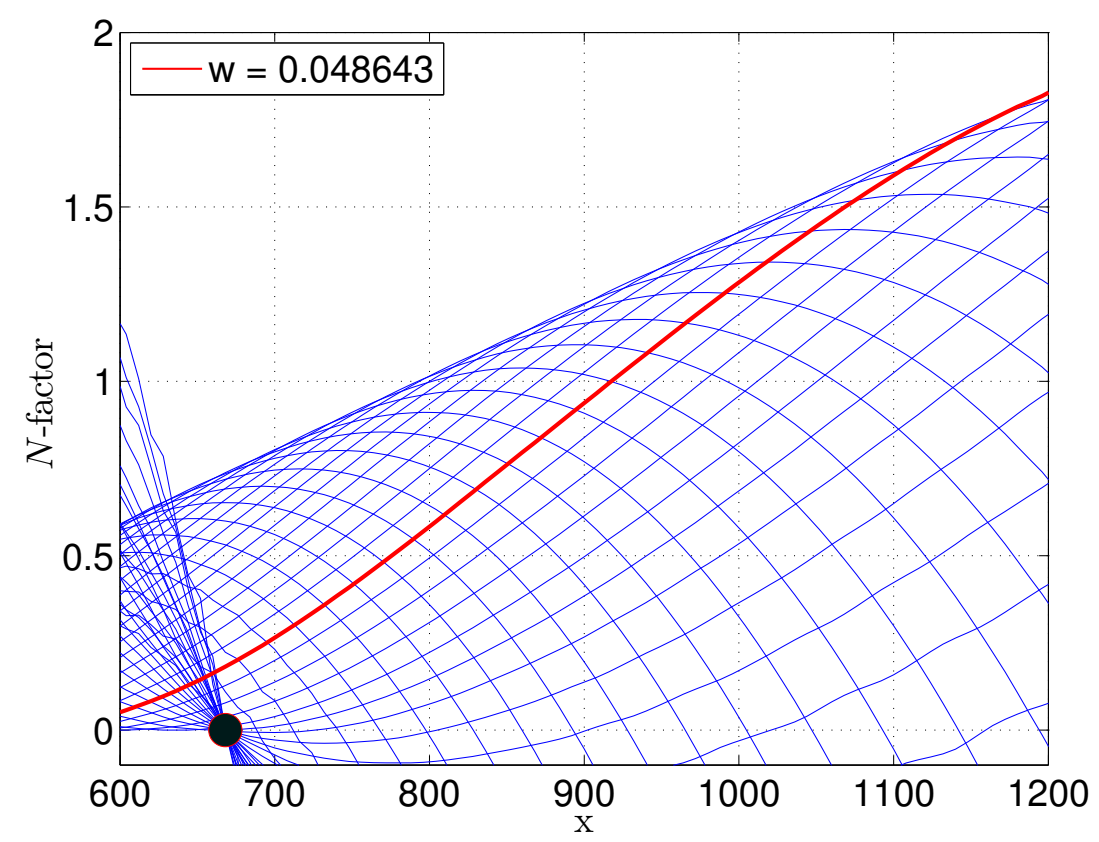

Figure 5.1: N-factor curves for Mach 0.7. The red line indicates the mode 2D most amplified, which in this case corresponds to $\omega=0.0486$.

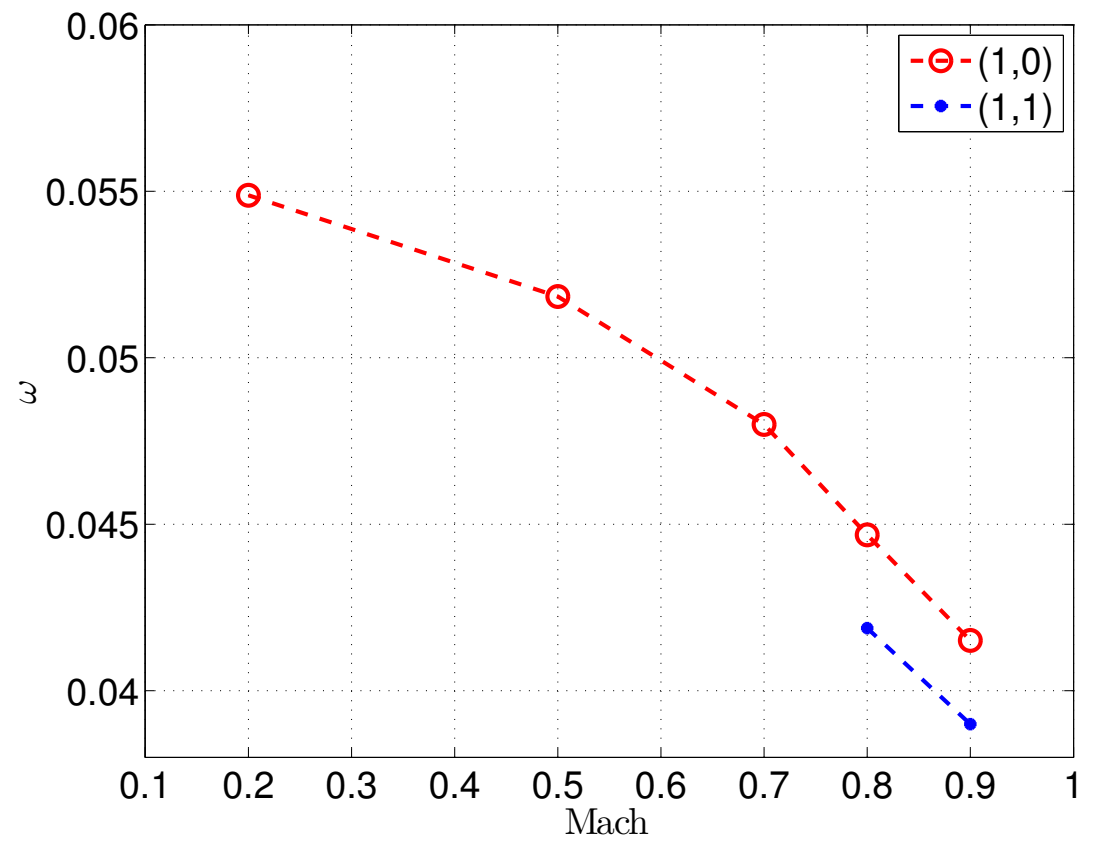

Figure 5.2: Variation of the frequency of most amplified mode with Mach number. From Mach 0.8, oblique modes around $\beta \sim 0.07$ are the linearly most unstable. 


\subsubsection{Nonlinear wave packet}

For the amplitude $a_{1}$, the linear and nonlinear wave packets are compared at an advanced downstream position in figure 5.3. The linear wave packet has a regular and smooth distribution of amplitude with no strong modulation in spanwise and streamwise. The nonlinear packet presents well defined three-dimensional structures, higher amplitudes are concentrated in a small region at the center of the packet. In figure 5.4 the spectral evolution is shown, for linear and nonlinear wave packet in global nondimensional variables. The linear amplification concentrates energy in the 2D modes, and, as occurs in the incompressible boundary layer, the frequency of the dominant mode decays downstream. In the nonlinear wave packet the spectrum gains energy, mainly in the subharmonic band and low frequency modes, a similar behavior was observed in the incompressible case (figure 4.37). For a more quantitative analysis the spectrum at the nonlinear position $x=972$ was selected. Figure 5.5 allows to separate clearly the linear from the nonlinear bands, by comparison of spectrum levels. From this spectrum a mode representative on each band was chosen, to compare its amplification curve with the linear wave packet (figure 5.6).

Each mode is identified in the figure by the color and the indices $(n, k)$ in the legend of the figure. Near the perturbation location, at $x=200$, the amplification curves of the nonlinear case follow the linear ones, because the amplitude disturbance was chosen to satisfy this condition. At downstream positions, all selected modes grows with higher growth rates than linear ones. For the considered computational domain, low frequency modes and the subharmonic band, denoted by the red circle, are the nonlinear dominant modes.
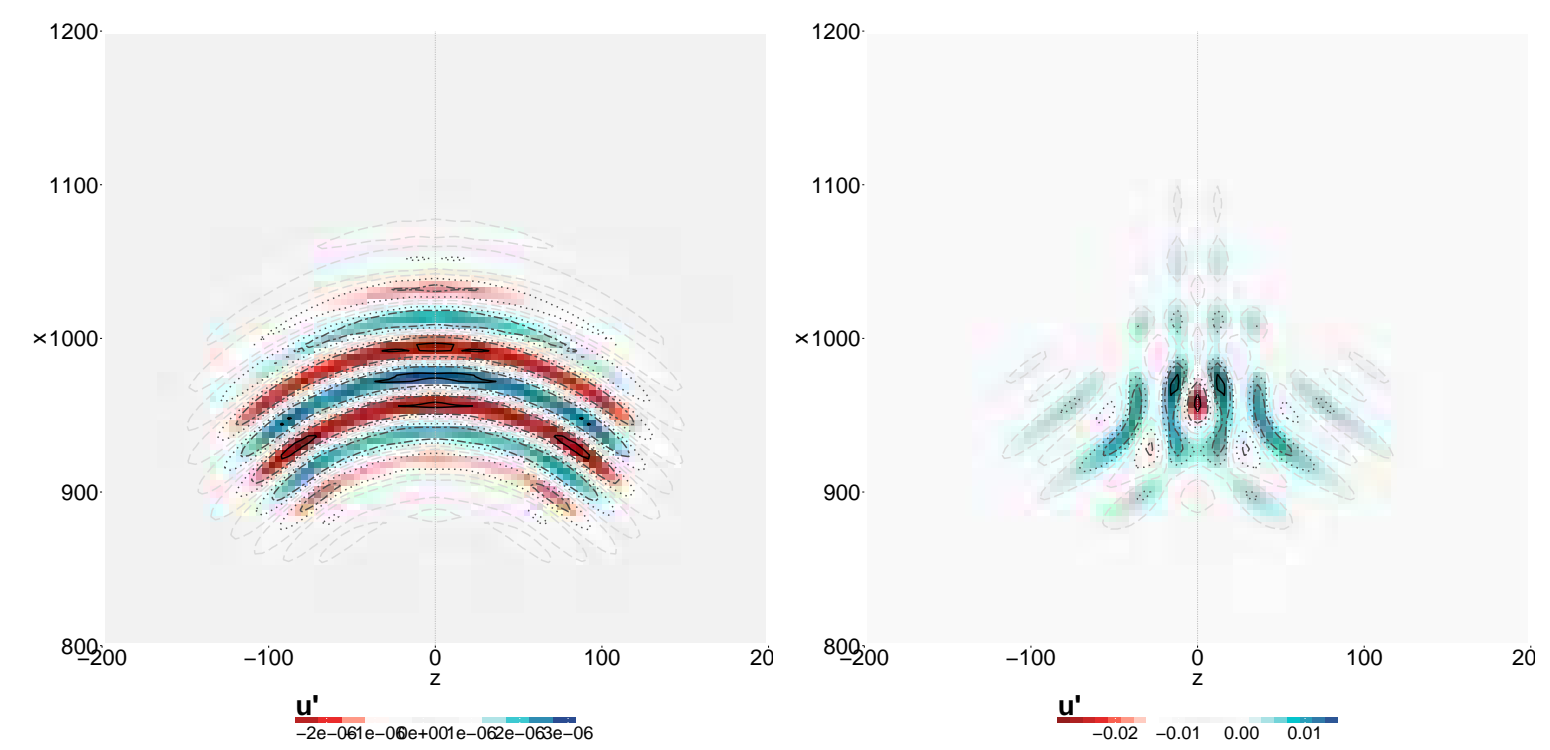

Figure 5.3: (a)Linear and (c) nonlinear wave packet at Mach 0.7, in $y=0.6 \delta^{*}(x)$. Contour levels at $90 \%(-), 50 \%(-.-), 30 \%(\ldots)$ and $10 \%(--)$ of the amplitude peaks. 

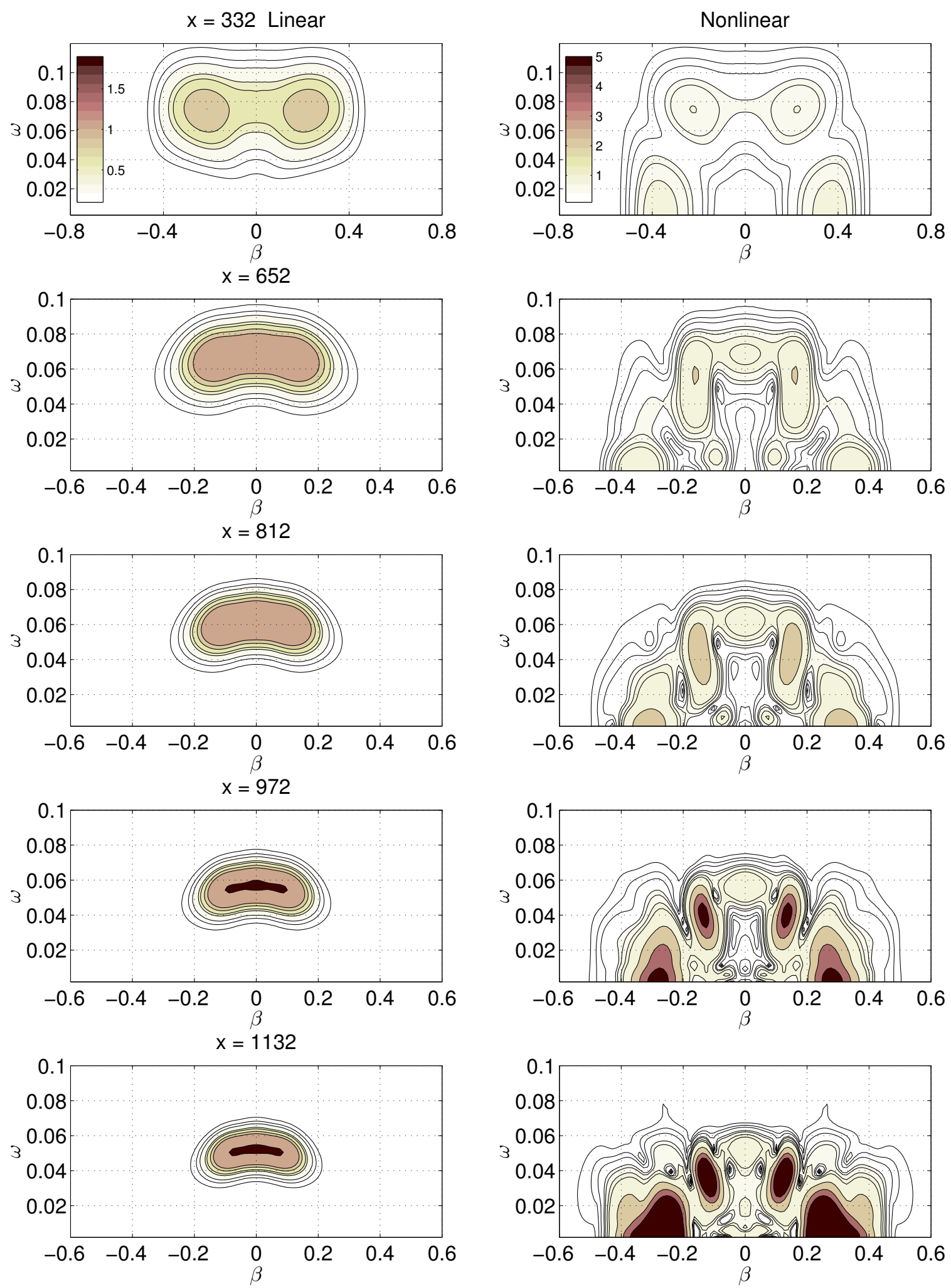

Figure 5.4: Spectral evolution at Mach 0.7, for linear and nonlinear wave packet, with $a_{1}=3.5 \times 10^{-5}$. 

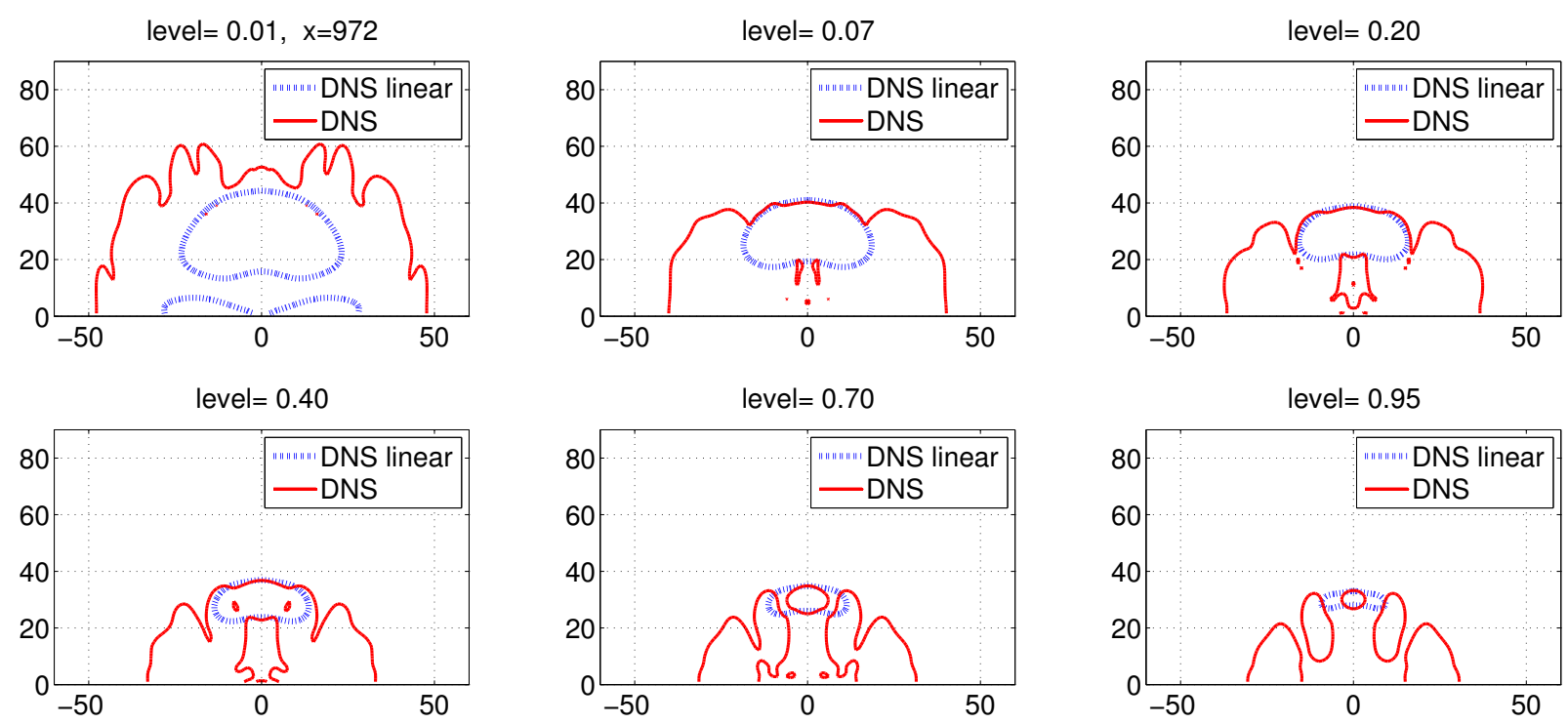

Figure 5.5: Spectrum levels for Mach 0.7 at $\mathrm{x}=972$.
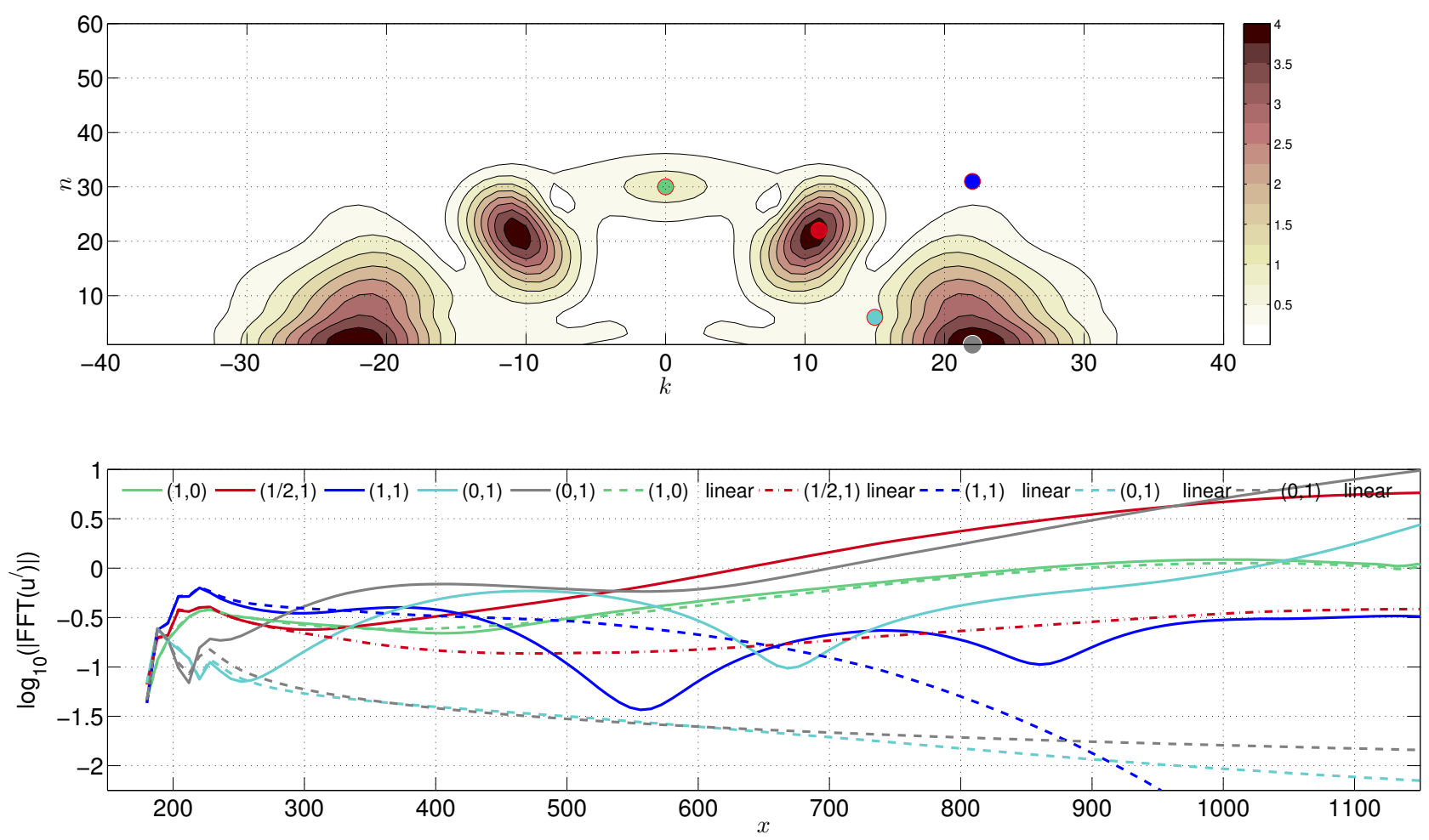

Figure 5.6: Amplification curves for Mach 0.7 for selected modes in the spectrum at the position $x=972$. All bands shows strong nonlinear amplification. 


\subsubsection{Amplitude effect}

To test effect of disturbance amplitude a packet with a lower amplitude than in previous case $a_{2}$, was simulated. A higher amplitude was not considered because it would be nonlinear from the source. The spectrum evolution (figure 5.7) and amplification curves (figure 5.8) reveal that in contrast to the case with higher amplitude, only two bands have nonlinear amplification. The subharmonic and low frequency modes, remain growing in the considered domain. The nonlinear fundamental band is much reduced in comparison with the higher amplitude case.

Velocity signals for the two nonlinear wave packets at the centerline are shown in figure 5.9. The packet with higher amplitude has stronger amplification and deformation relative to the linear and the lower amplitude ones. The amplitude has an important role in the nonlinear mechanisms that are triggered in the transition region.
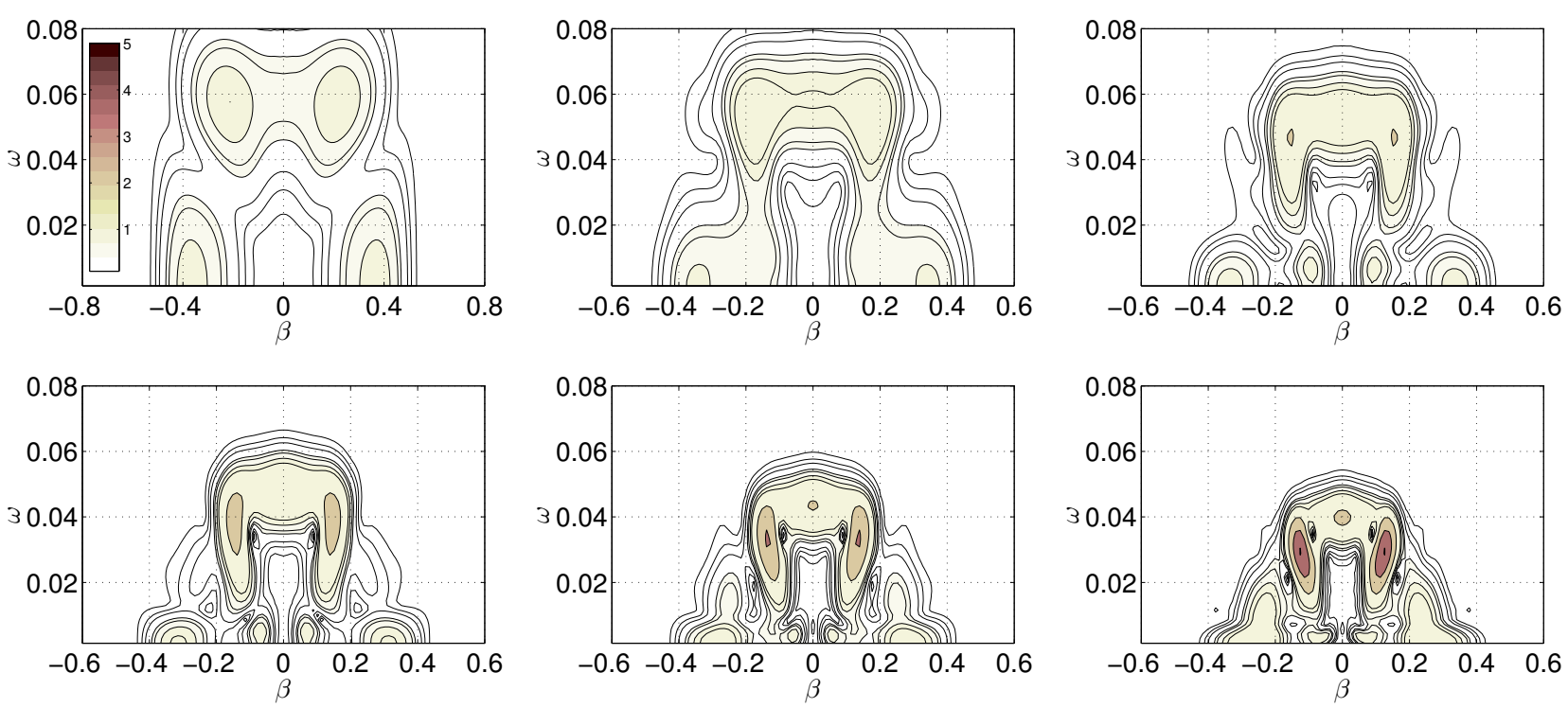

Figure 5.7: Spectral evolution for Mach 0.7, for nonlinear wave packet with disturbance amplitude $a_{2}=2.5 * 10^{-5}$. 

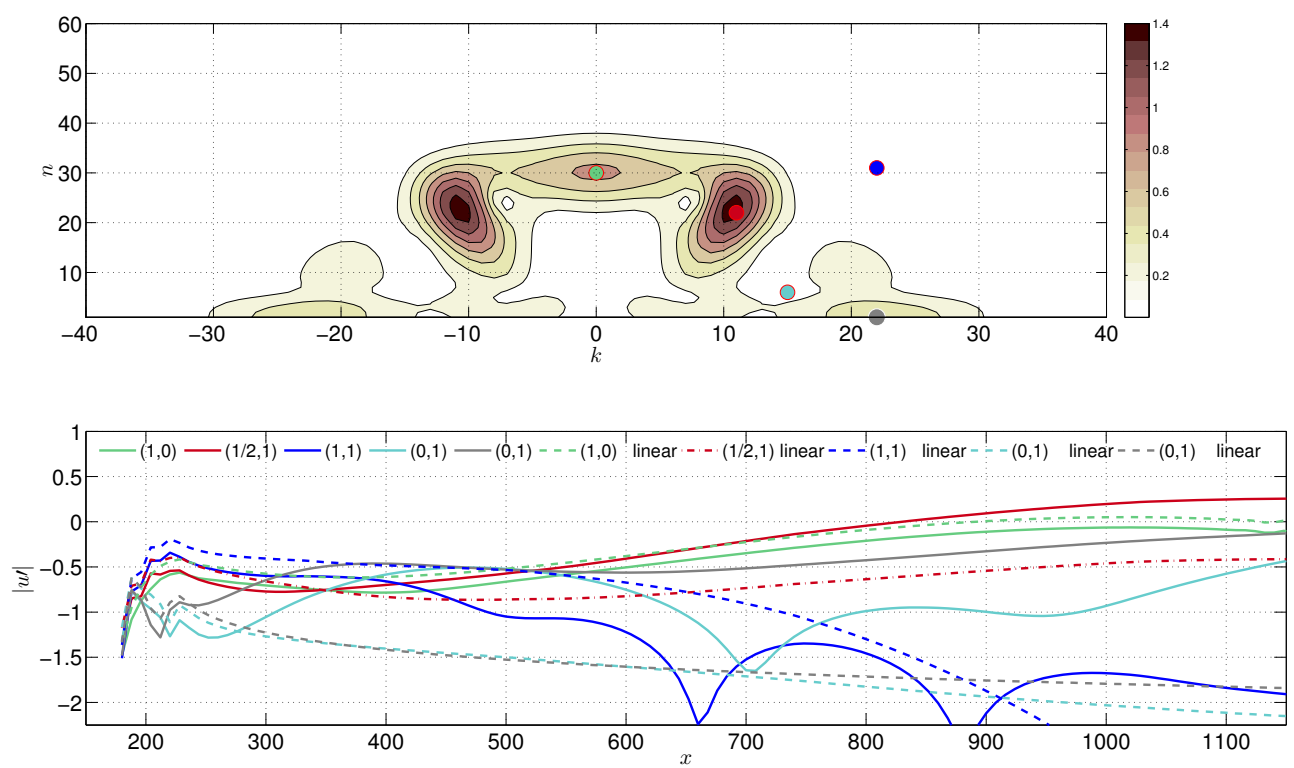

Figure 5.8: Amplification curves for disturbance with $a_{2}=2.5 * 10^{-5}$.

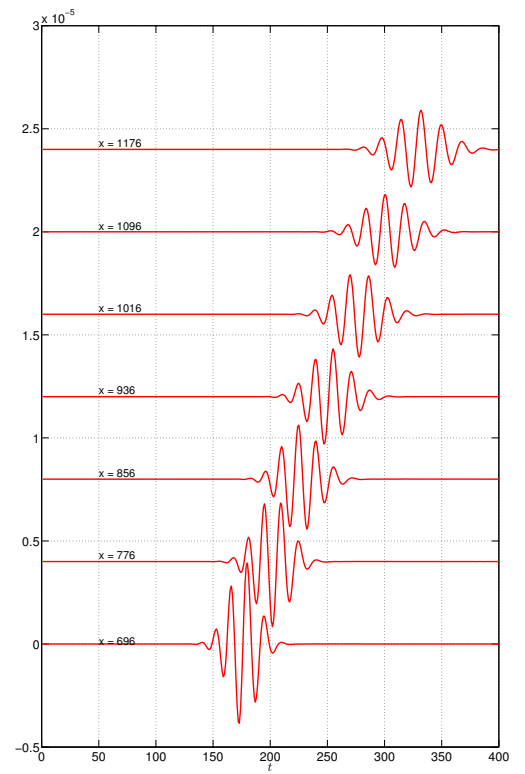

(a)

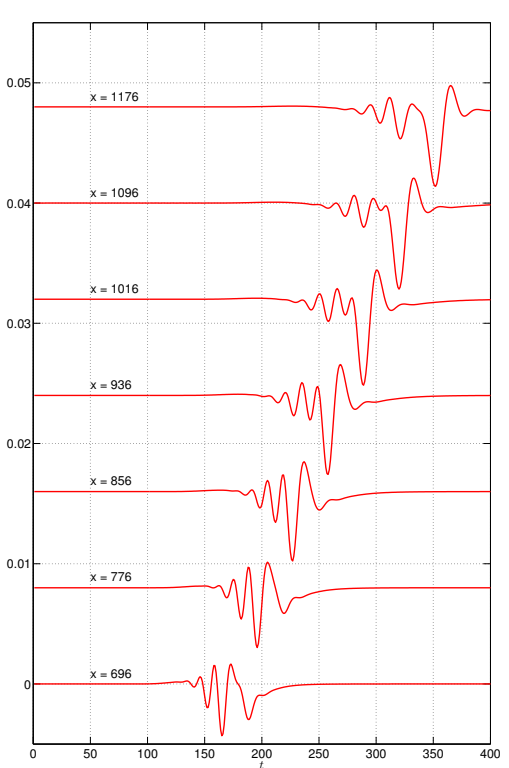

(b)

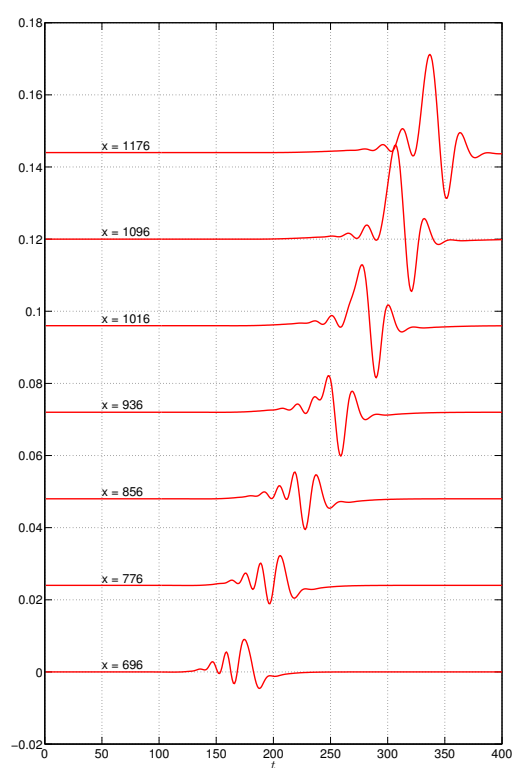

(c)

Figure 5.9: u component of the velocity at the centerline. Amplitude effect on the nonlinear wave packet evolution at Mach 0.7, (a) linear wave packet, (b) $a_{2}=2.5 \times 10^{-5}$ and (c) $a_{1}=3.5 \times 10^{-5}$ 


\subsubsection{Analysis of possible secondary instability regime}

To verify if the subharmonic band has a behavior similar to that observed in the incompressible boundary layer, discussed in section 4.4.1, two modes were selected from the spectrum at the position $x=972$, as shown in figure 5.10. The amplification curves for the selected modes in figure 5.10(a) reveal a strong deviation from the linear growth rate at the position $x \sim 280$, that remains decaying. The oblique mode $(1 / 2,1)$ is amplified nonlinearly up to position $x \sim 800$, here it recovers the linear growth rate. Changes in the growth rates for the oblique mode are driven by the threshold amplitude of the 2D wave, as indicated by dashed lines. The mode shown in figure 5.10(b) also presents nonlinear amplification, but not recovers the linear growth rate when the correspondent fundamental wave reach the threshold amplitude, even it remains growing. This fact suggest the presence of another nonlinear amplification source.

In the fundamental band, the same analysis was carried out for a pair of modes (figure 5.11) to verify the presence of K-type mechanism. Threshold amplitudes of the driving $2 \mathrm{D}$ wave show that amplification do not corresponds with this mode. Nonlinear amplification could be generated by subharmonic or detuned subharmonic resonance, and influenced by nonlinear distortions, as was observed in the incompressible case, (see section 4.4.1).
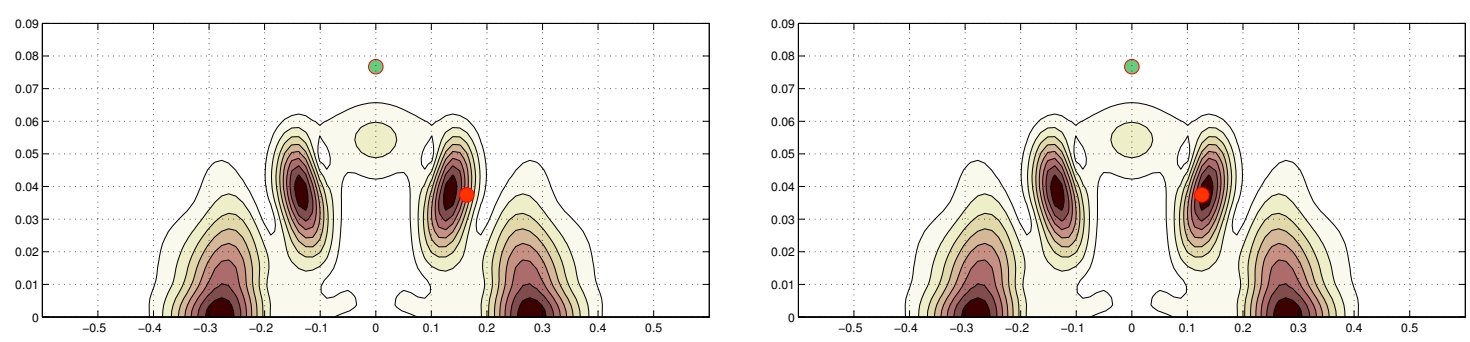

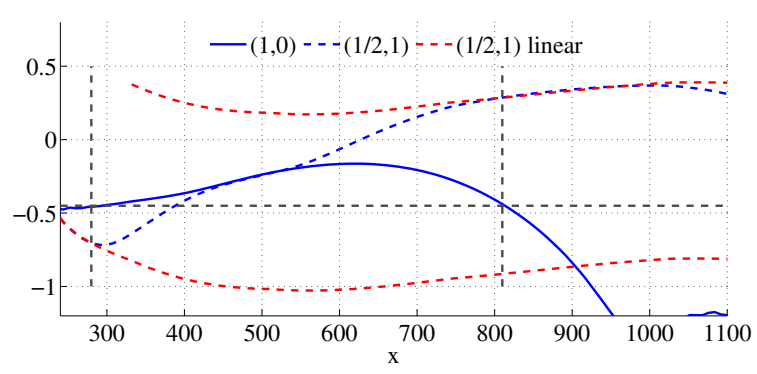

(a)

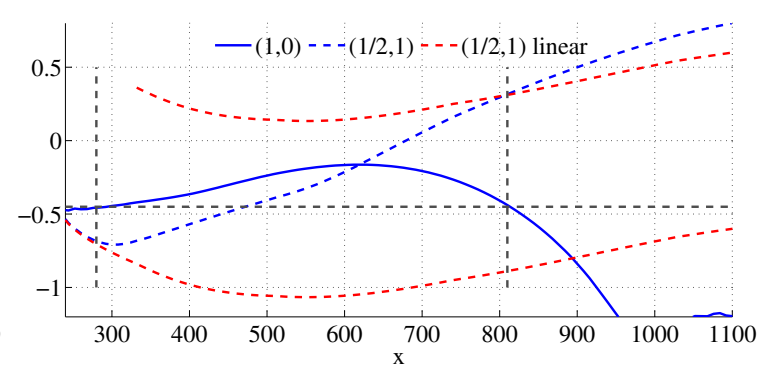

(b)

Figure 5.10: Spectrum at the position $x=972$, for the wave packet generated with amplitude $a_{1}=3.5 * 10^{-5}$. (a) Most amplified subharmonic mode (b) non identified resonance, could be a detuned resonance. 

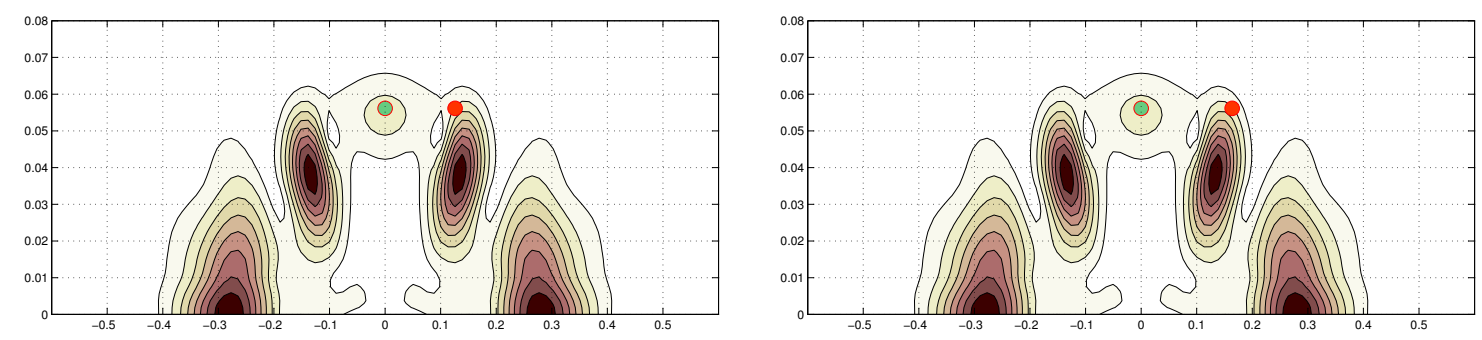

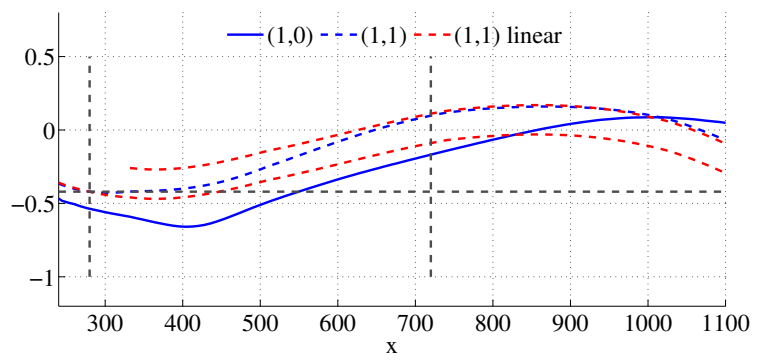

(a)

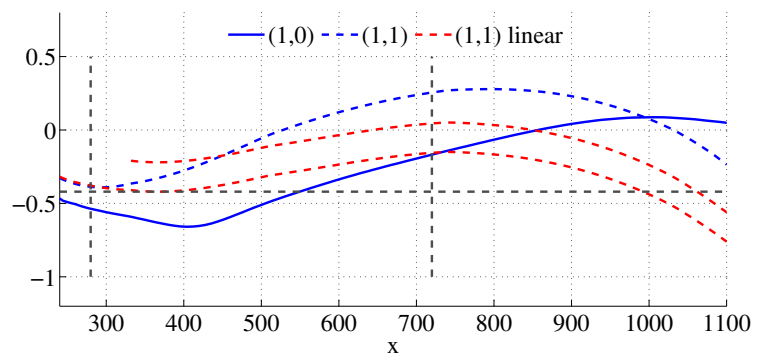

(b)

Figure 5.11: (a) The oblique mode $(1,1)$ does not resonates with the fundamental mode $(1,1)$. (b) Non identified resonance. $a_{2}=3.5 \times 10^{-5}$.

\subsection{Wave packet in a boundary layer at Mach 0.9}

As in the previous case, a linear and a nonlinear wave packet were simulated, with isothermal wall, $T_{\text {wall }}=1$, at Mach 0.9 . This Mach number is interesting because it is typical for transport aircraft at cruise.

\subsubsection{Linear wave packet}

From DNS simulations, it was found that at Mach 0.9, the linear growth rates are around 10 times lower than growth rates for incompressible boundary layer for $\omega 0.05$. Hence, the boundary layer presents a longer transition region. Comparison (figure 5.12) for linear wave packet at Mach 0.2 and Mach 0.9 at the centerline shows that, while for the incompressible boundary layer the wave packet already amplifies, in the compressible case the packet decays.

In the physical space (figure 5.13), the wave packet has low modulation in spanwise, but more intense than in the incompressible regime, as can be observed in figure 5.16. The spectral evolution of the linear wave packet in global nondimensional variables (figure $5.14)$, reveals that in contrast with all the previous cases, the most linear unstable modes are oblique, centered around the mode $\omega=0.41$ and $\beta=0.1$, which generates a higher modulation of the wave packet in spanwise direction and is associated with the more curved packet shape. 

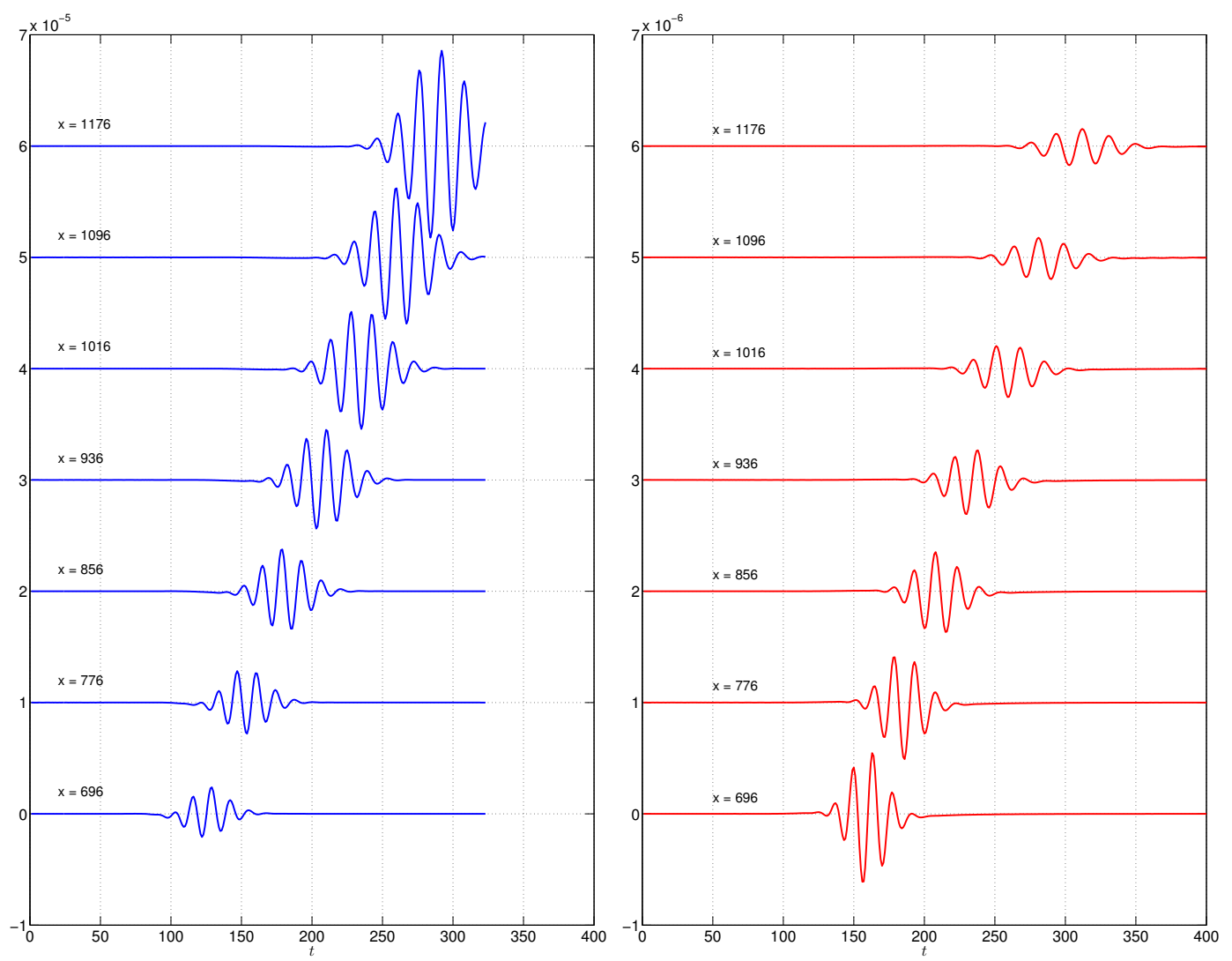

Figure 5.12: Comparison of linear wave packet for (a) Mach 0.2 and (b) Mach 0.9 at the centerline.

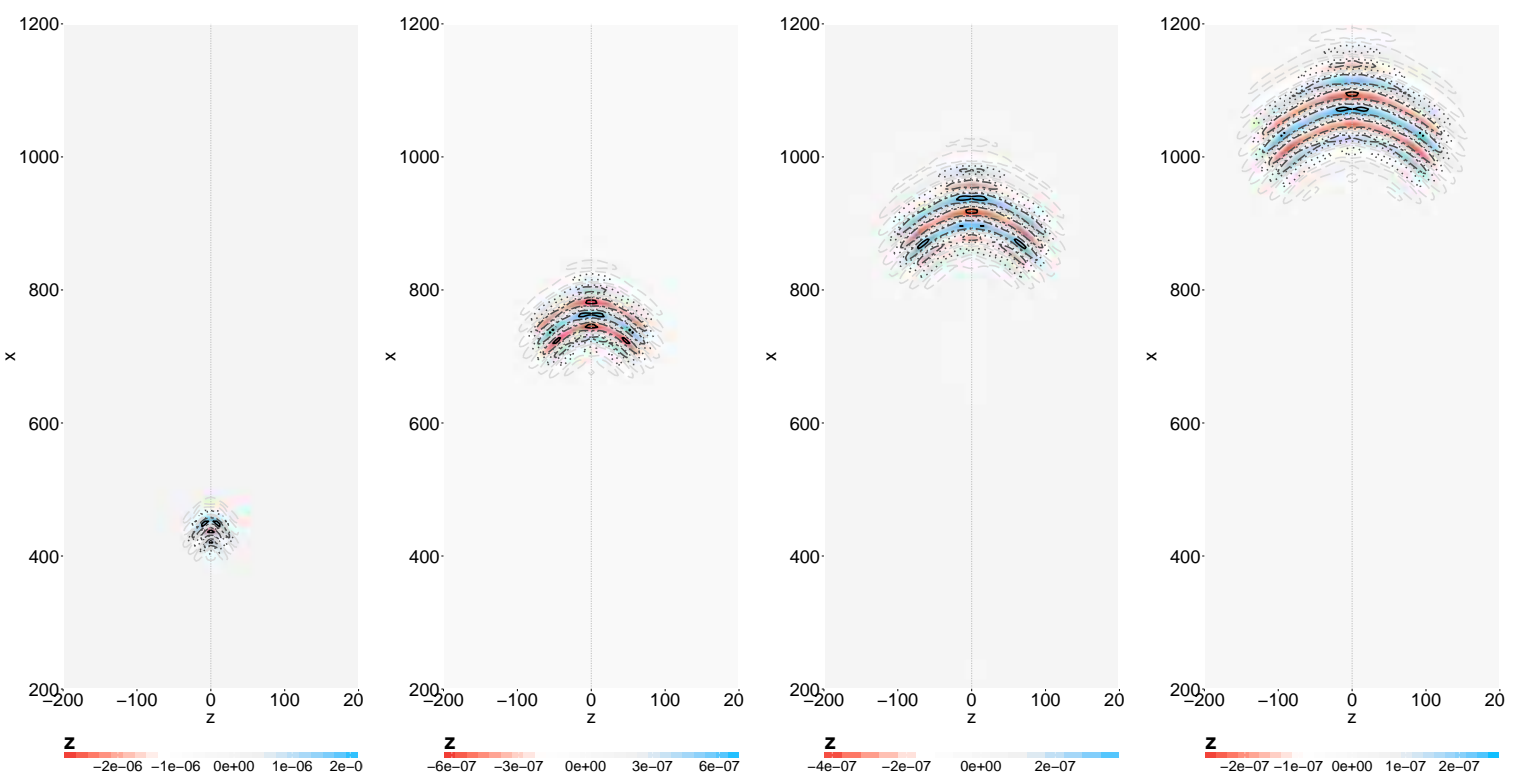

Figure 5.13: Linear wavepacket at Mach 0.9 , in $y=0.6 \delta^{*}(x)$. Contour levels at $90 \%(-)$, $50 \%(-.-), 30 \%(\ldots)$ and $10 \%(--)$ of the amplitude peaks in the packet. 

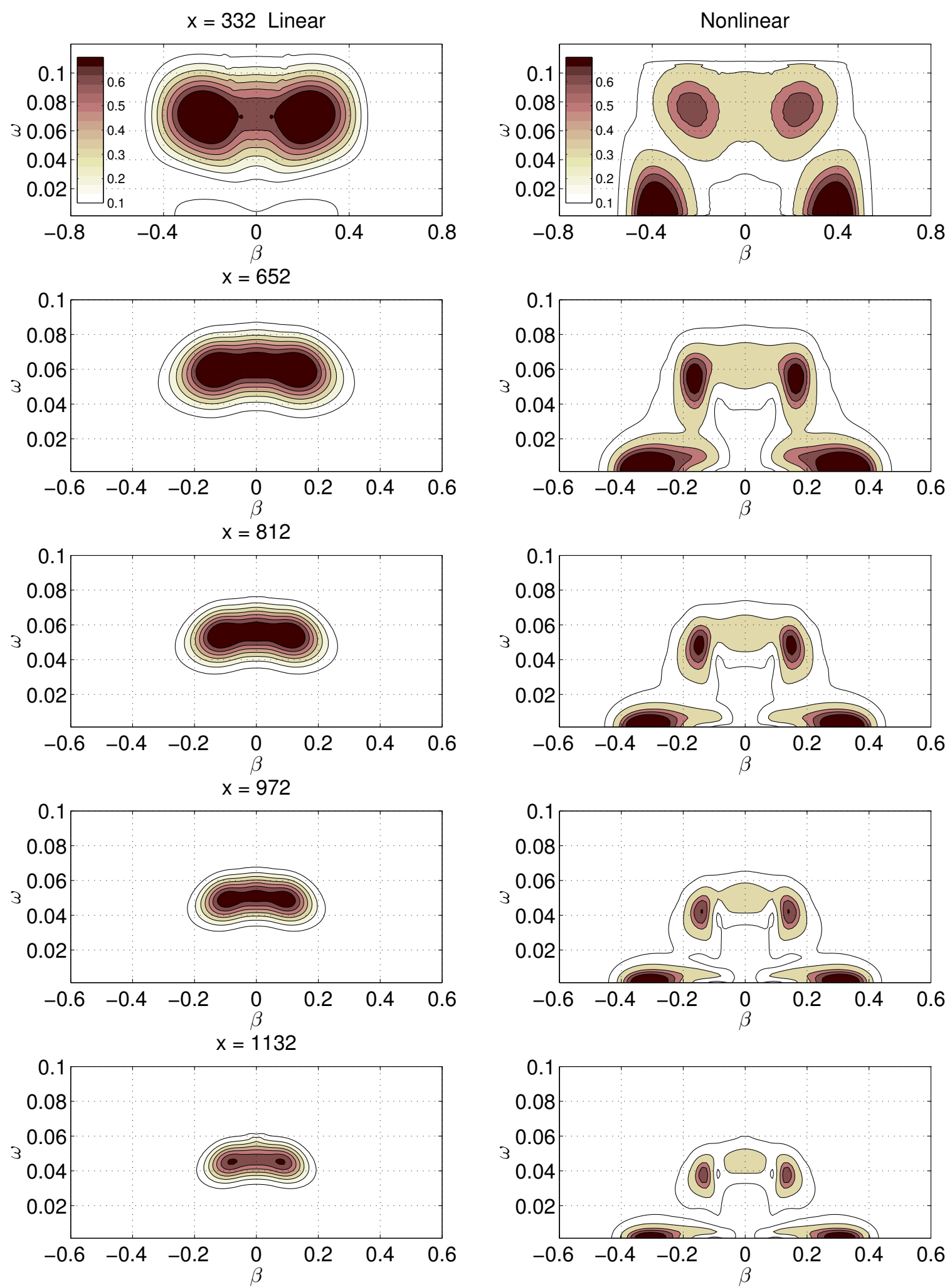

Figure 5.14: Comparison of linear and nonlinear spectrum evolution at Mach 0.9 


\subsubsection{Nonlinear wave packet}

Evolution in the physical space (figure 5.15) shows three-dimensional structures, with strong streaks located at the center of the packet and oblique waves, as was observed in the nonlinear case for Mach 0.7. In figure 5.16 linear and nonlinear wave packets are compared, and a group of oblique waves with a propagation angle $\psi \sim 45^{0}$ can be identified. In the spectral evolution (figure 5.14) only two bands presents nonlinear amplification, subharmonic and low frequency modes. From figure 5.17 the linear and nonlinear bands can be separated easily. A more precise quantitative analysis can be done from the amplification curves shown in figure 5.18, which corresponds to the spectrum at the position $x=1132$, by selecting representative modes to be tracked along the evolution. Amplitude of nonlinear wave packet was chosen to be linear in a region next to the perturbation region. There are two bands of modes that amplify nonlinearly, low frequency modes, responsible for strong streaks and the subharmonic band. Low frequency modes, are strong near the perturbation source, but eventually decay, with the linear growth rate, as can be observed in the figure, by the superposition of the linear amplification curve in the last part of the domain. Only the subharmonic modes amplifies nonlinearly and in sustained throughout the evolution. Yet, from the spectral evolution, figure 5.18 shows that the global behavior is to decay. To investigate this aspect, the domain of the simulation was extended in spanwise and streamwise, up to $x=2500$. The resulting spectral evolution is shown in figure 5.19, and the previous behavior remains, also from the position $R e / x \sim 1100$ the subharmonic modes begin to dominate the transition process, as can be seen in the amplification curves for the extended domain, (see figure 5.20).

Finally the phase locking condition is shown in figure 5.21, for the selected mode, Craik condition is satisfied, hence the nonlinear locking is not evident. 


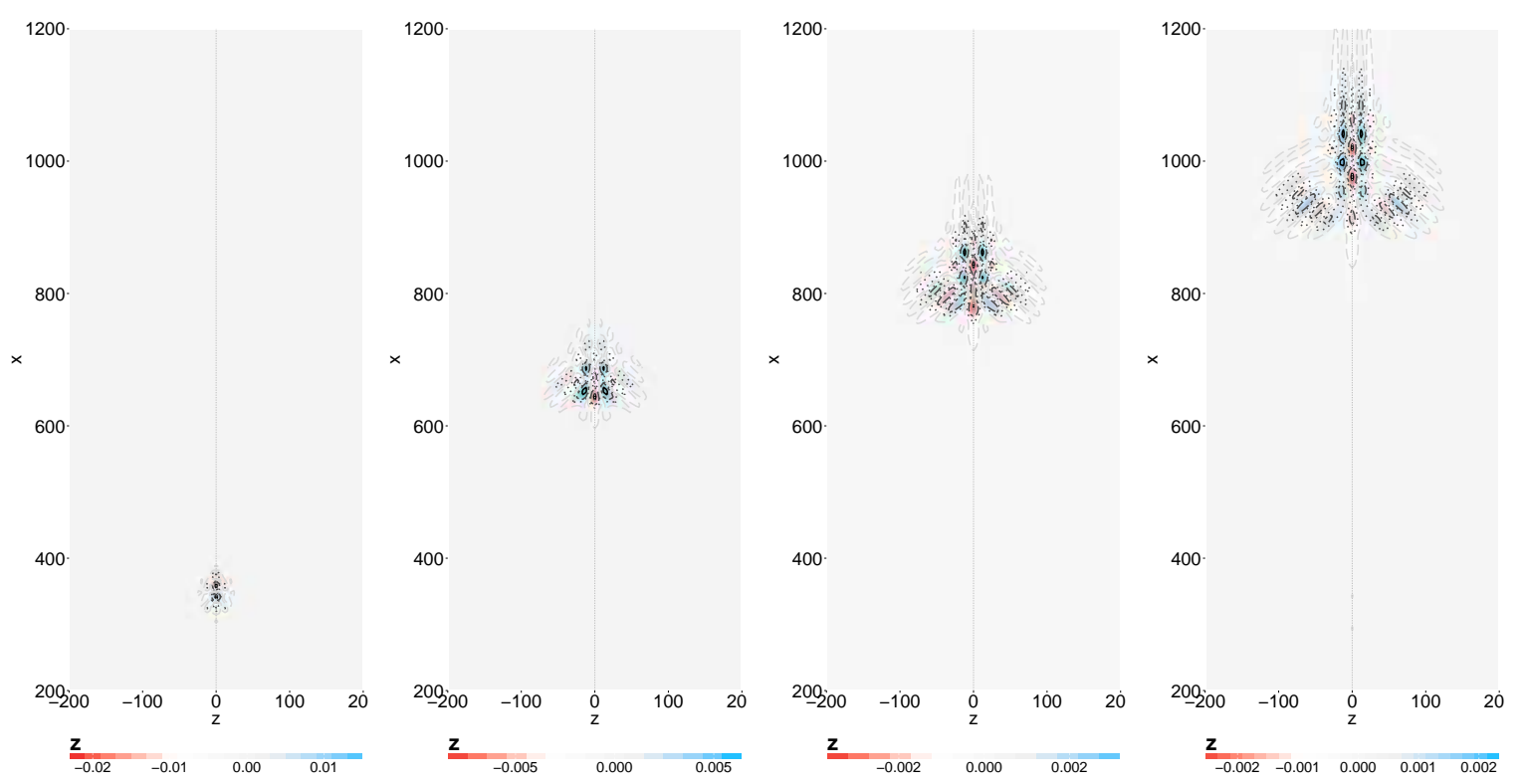

Figure 5.15: Nonlinear wavepacket at Mach 0.9, in $y=0.6 \delta^{*}(x)$. Contour levels at $90 \%$ $(-), 50 \%(-.-), 30 \%(\ldots)$ and $10 \%(--)$ of the amplitude peaks in the packet.

(a)

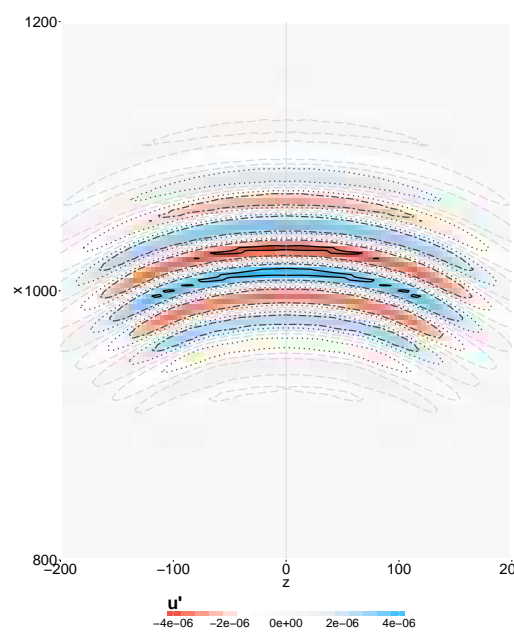

(b)

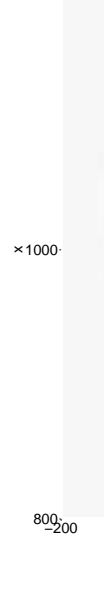

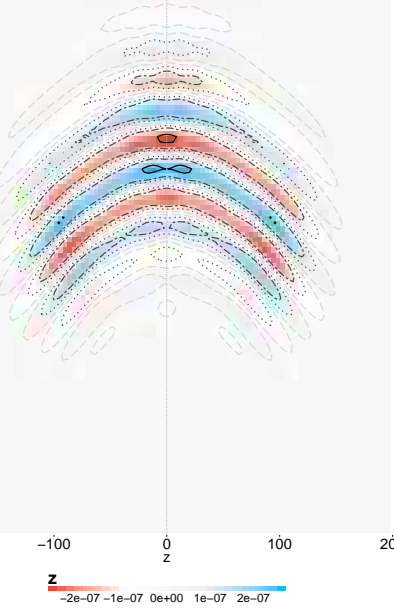

(c)

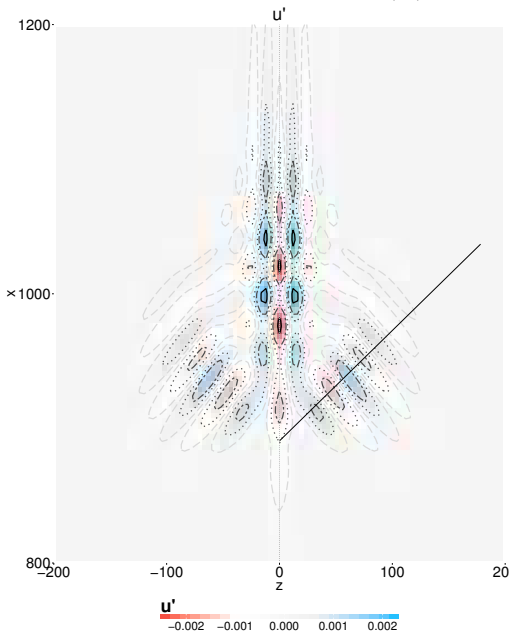

Figure 5.16: Linear wave packet in incompressible boundary layer $($ Mach $=0.2)$ (a) and (b) at Mach 0.9. (c) Nonlinear wavepacket at Mach 0.9, in $y=0.6 \delta^{*}(x)$. Contour levels at $90 \%(-), 50 \%(-.-), 30 \%(\ldots)$ and $10 \%(--)$ of the amplitude peaks in the packet. 

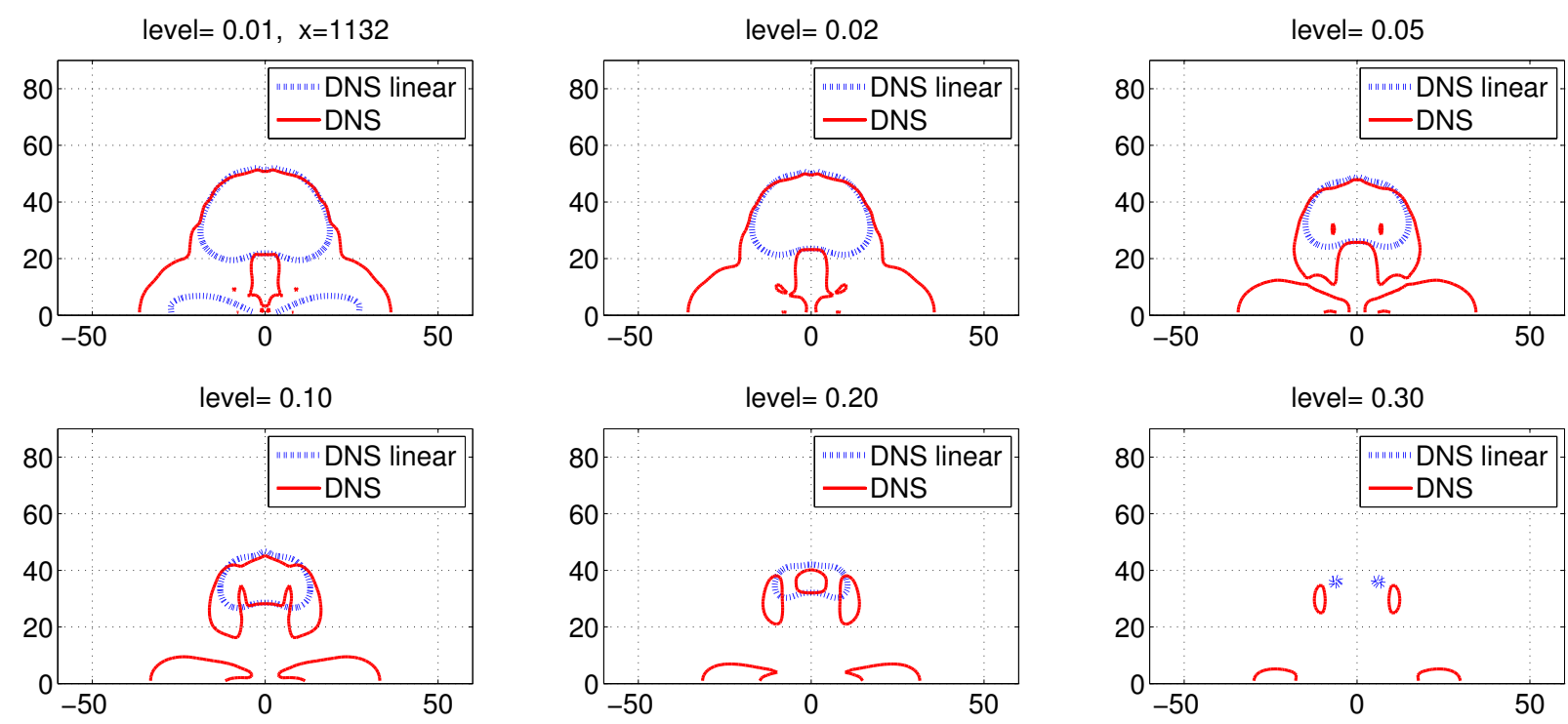

Figure 5.17: Comparison of linear and nonlinear spectrum levels at Mach 0.9 in $\mathrm{x}=1132$.
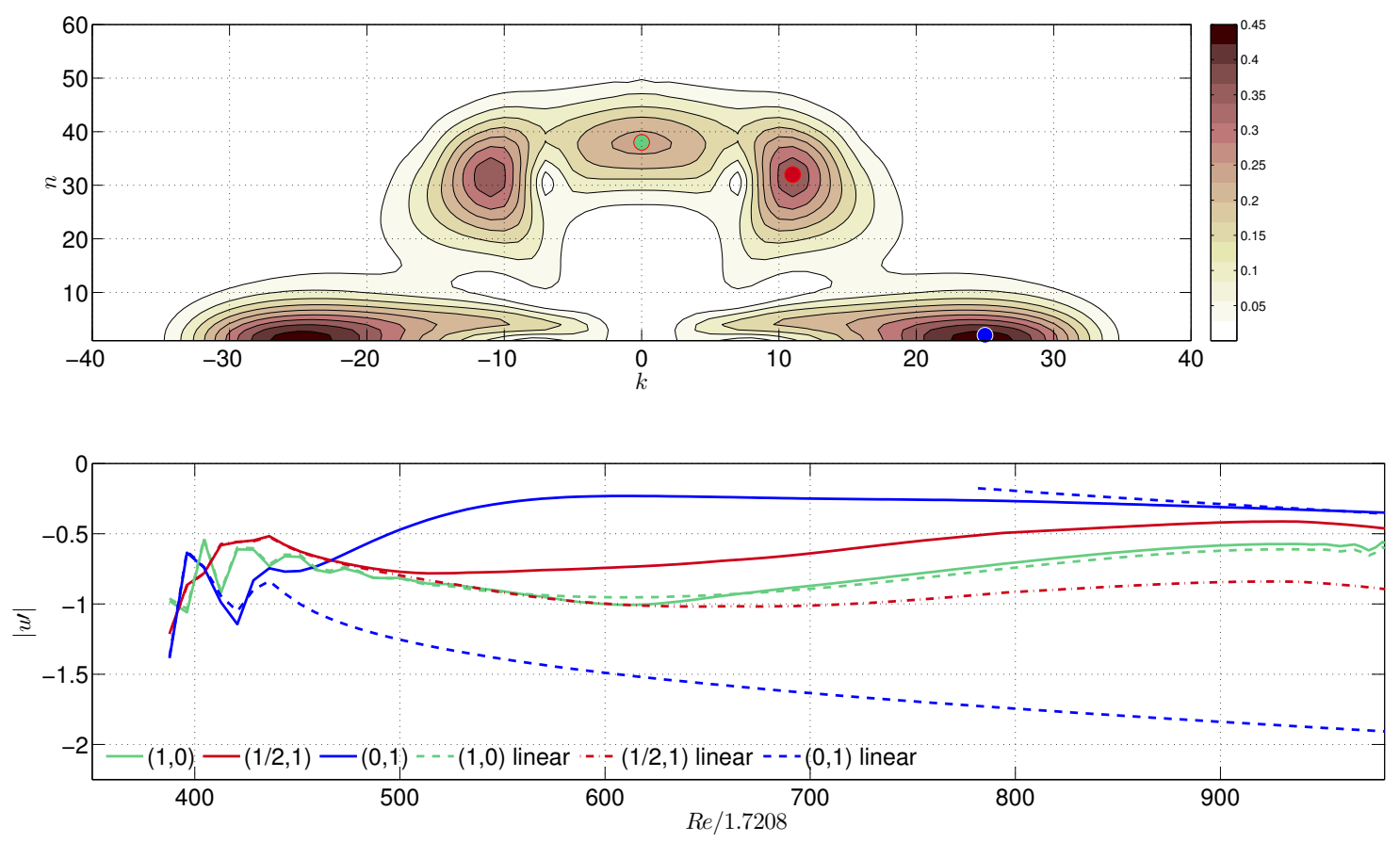

Figure 5.18: Amplification curves at $\mathrm{x}=1132$. Streaks are dominant from the beginning, but eventually decay and reach the linear growth rate. Subharmonic mode remains amplifying nonlinearly. 

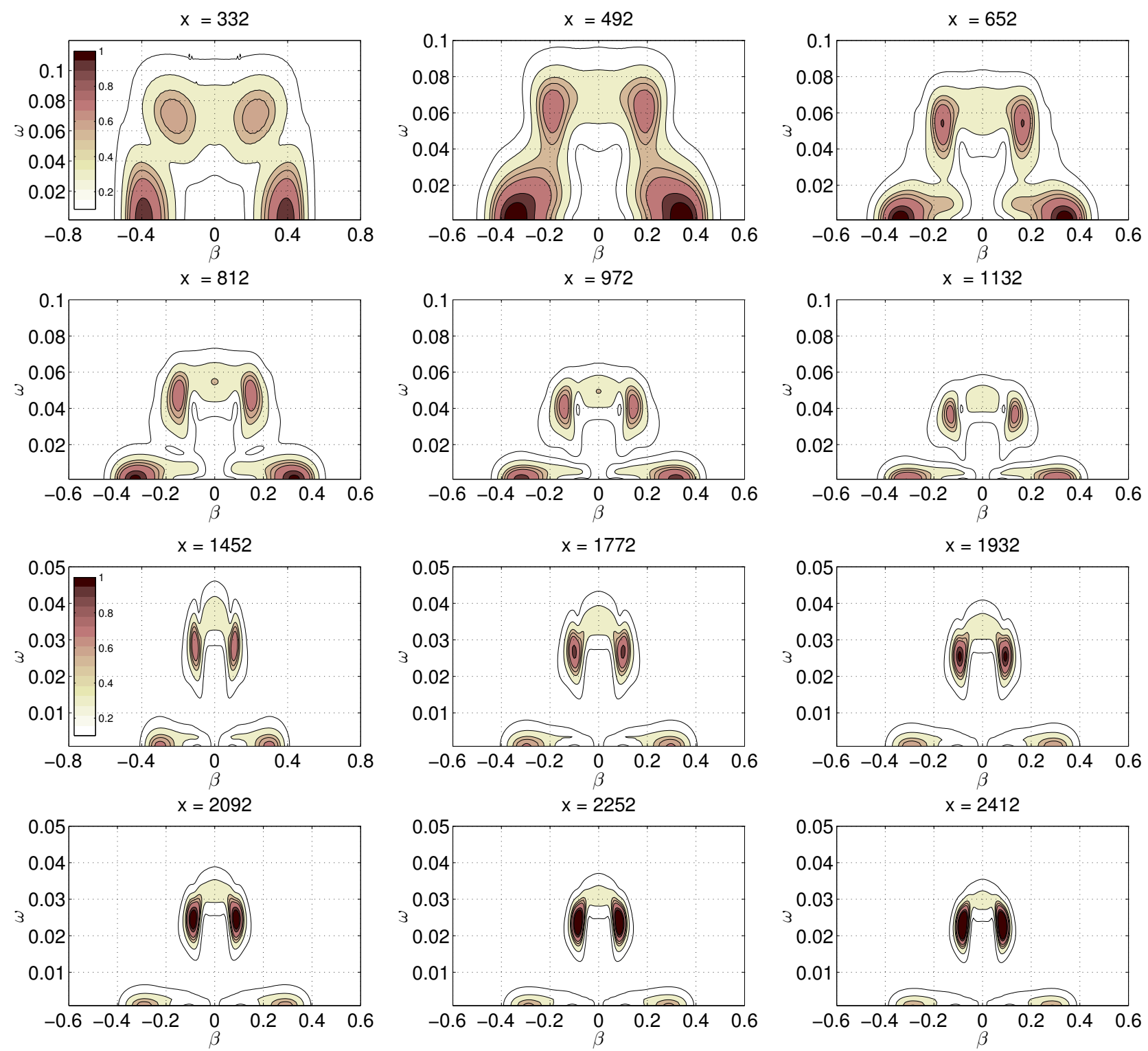

Figure 5.19: Spectrum evolution of nonlinear wave packet at Mach 0.9 in extended domain in streamwise direction, with $x_{\max }=2500$. 

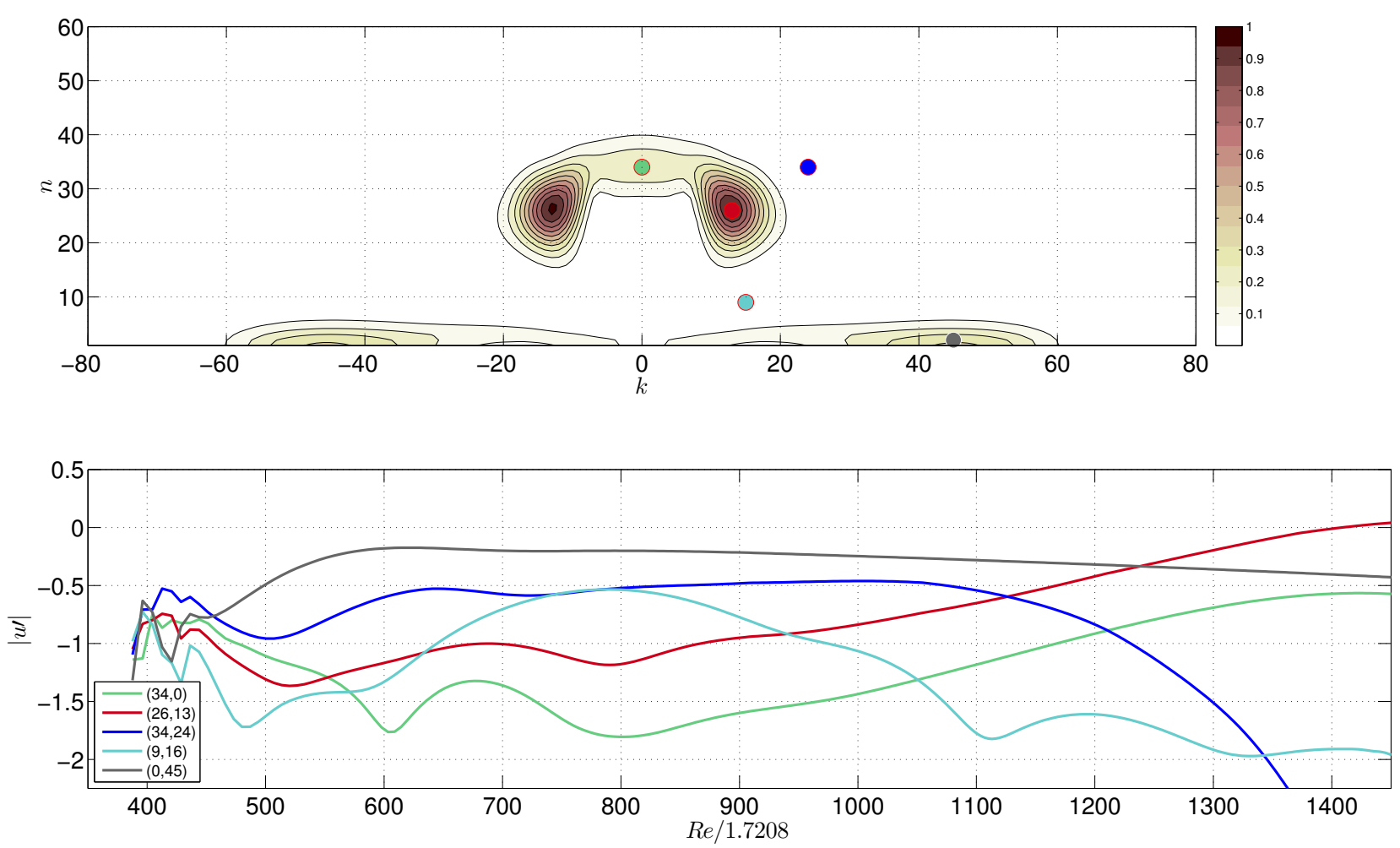

Figure 5.20: Amplification curve at $\mathrm{x}=2412$. Streaks are dominant but decay in downstream.
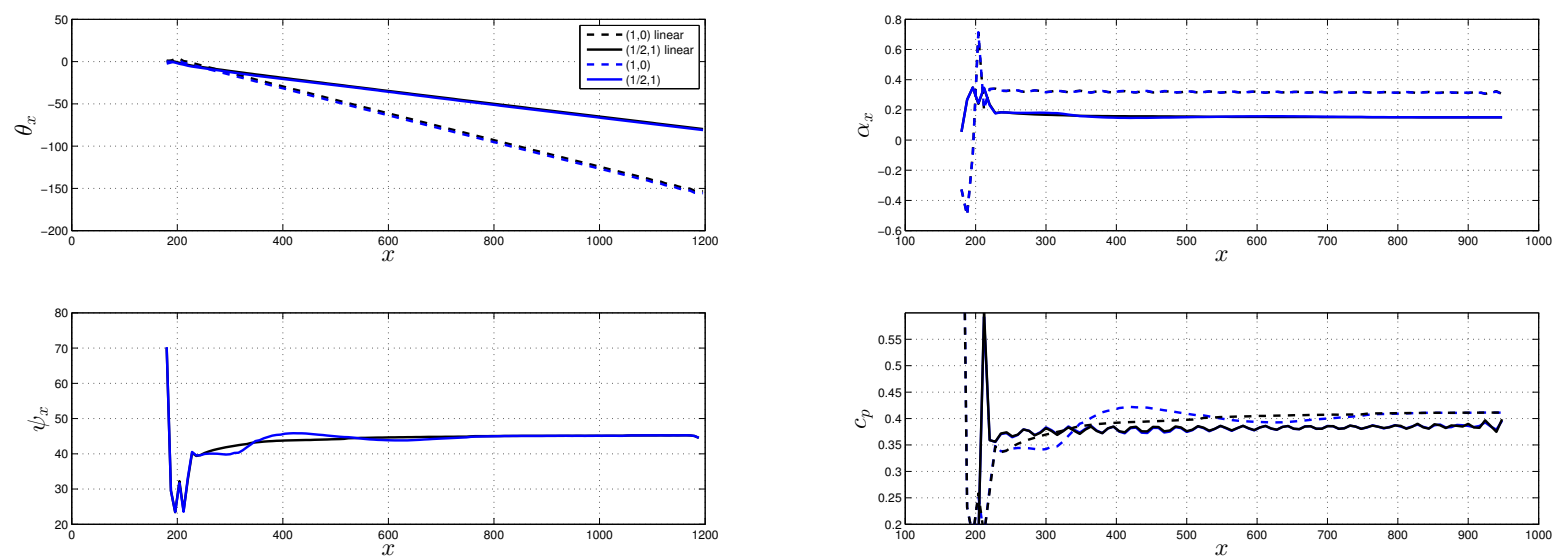

Figure 5.21: Phase locking for subharmonic mode. 


\subsection{Interaction between wavepackets in a boundary layer at Mach 0.9}

In the Mach 0.9 boundary layer, the nonlinear stage of the wave packet occupies a larger region than in the incompressible boundary layer, hence, the wave packet can spread along the domain mainly, in spanwise direction, which is a favorable factor for wave packet spanwise interaction. An isolated packet may represent a rare scenario in natural transition, spanwise interaction of packets could be more realistic model and in the literature there are no results for this kind of problem. Interaction between wave packets may be a destabilizing factor.

For the wave packet interaction study, two cases were considered, a pair of wave packets with two different separations, to represent approximately $50 \%$ of overlap in the linear region and $50 \%$ in the nonlinear region. The distances between packet centers were $d=30$ and $d=60$ respectively. The computational domain and flow parameters were maintained. For each case, the corresponding pair of low amplitude (linear) wave packets were generated by the superposition in the physical space of two isolated packets, to be used as reference case.

\subsubsection{Pair of wave packets separated by $d=30$}

In physical space, the evolution for the linear pair of wave packets is shown in figures 5.22 and 5.23 for the nonlinear pair. At the initial positions the two packets can be easily identified. Further downstream, they are joined in a unique structure, with a stronger modulation than the isolated packet. The nonlinear pair of wave packets develops strong streaks, with higher amplitude than in isolated wave packet.

The spectral evolution for the linear and the nonlinear cases (figure 5.24) shows amplification for bands centered around specific modes. In the nonlinear case, strong steady streaks are amplified. In particular, the mode $(\omega=0.045, \beta=0.16)$, seems to generate the harmonic $(0,0.32)$, that corresponds to the amplification of the mode, $(0,2 \beta)$, as occurs in the oblique transition.

From comparison of spectrum levels at $x=1132$, linear and nonlinear bands easily can be identified (figure 5.25) . The amplification curves were calculated for selected modes (figure 5.26) in several bands. Steady streaks display sustained nonlinear growth along the evolution. In general, it is observed a destabilizing effect by the wave packet interaction, because modes in the others bands have nonlinear amplification, in contrast to the observations on the isolated wave packet. 


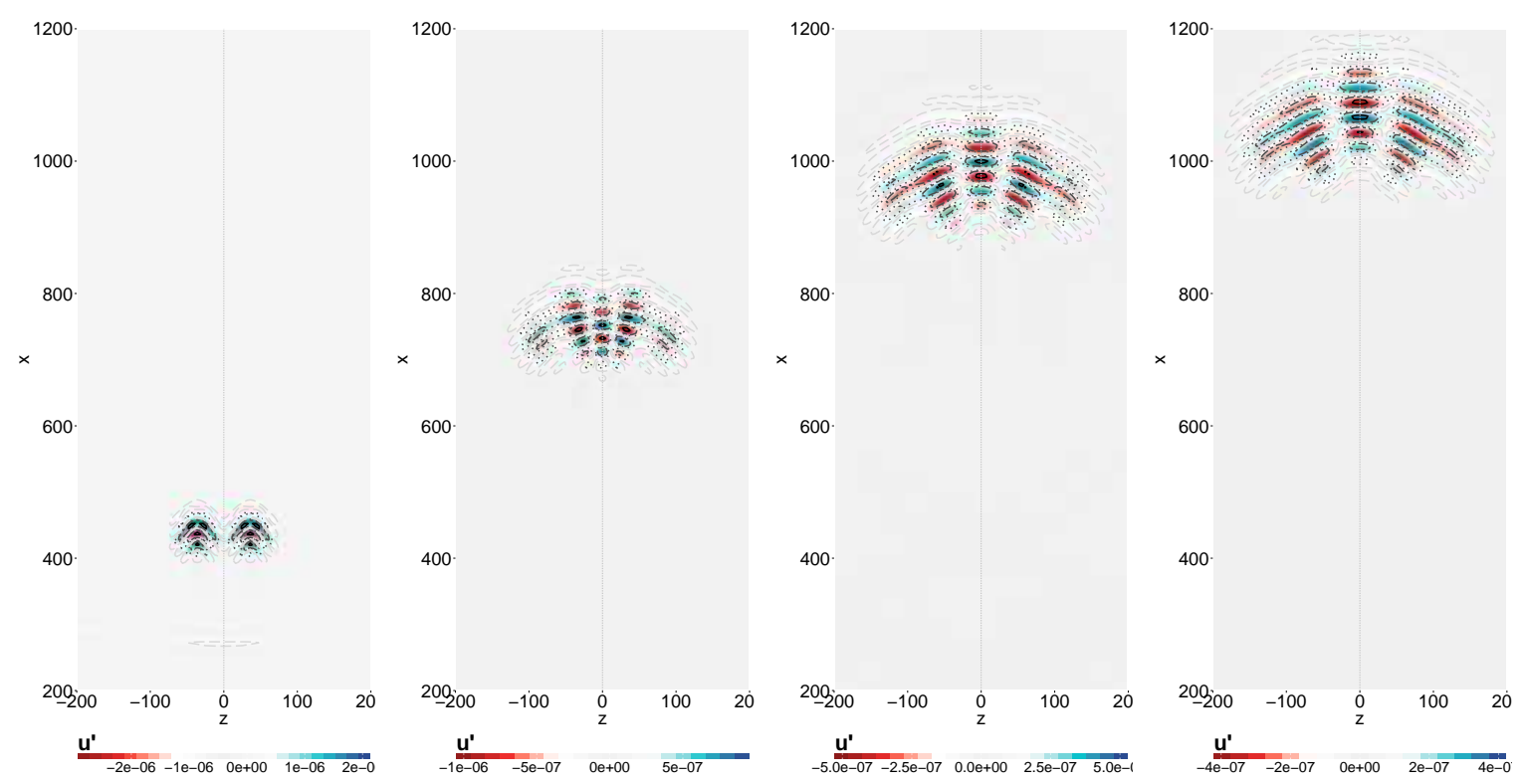

Figure 5.22: Linear evolution in physical of a pair of wave packets separated by $d=30$.
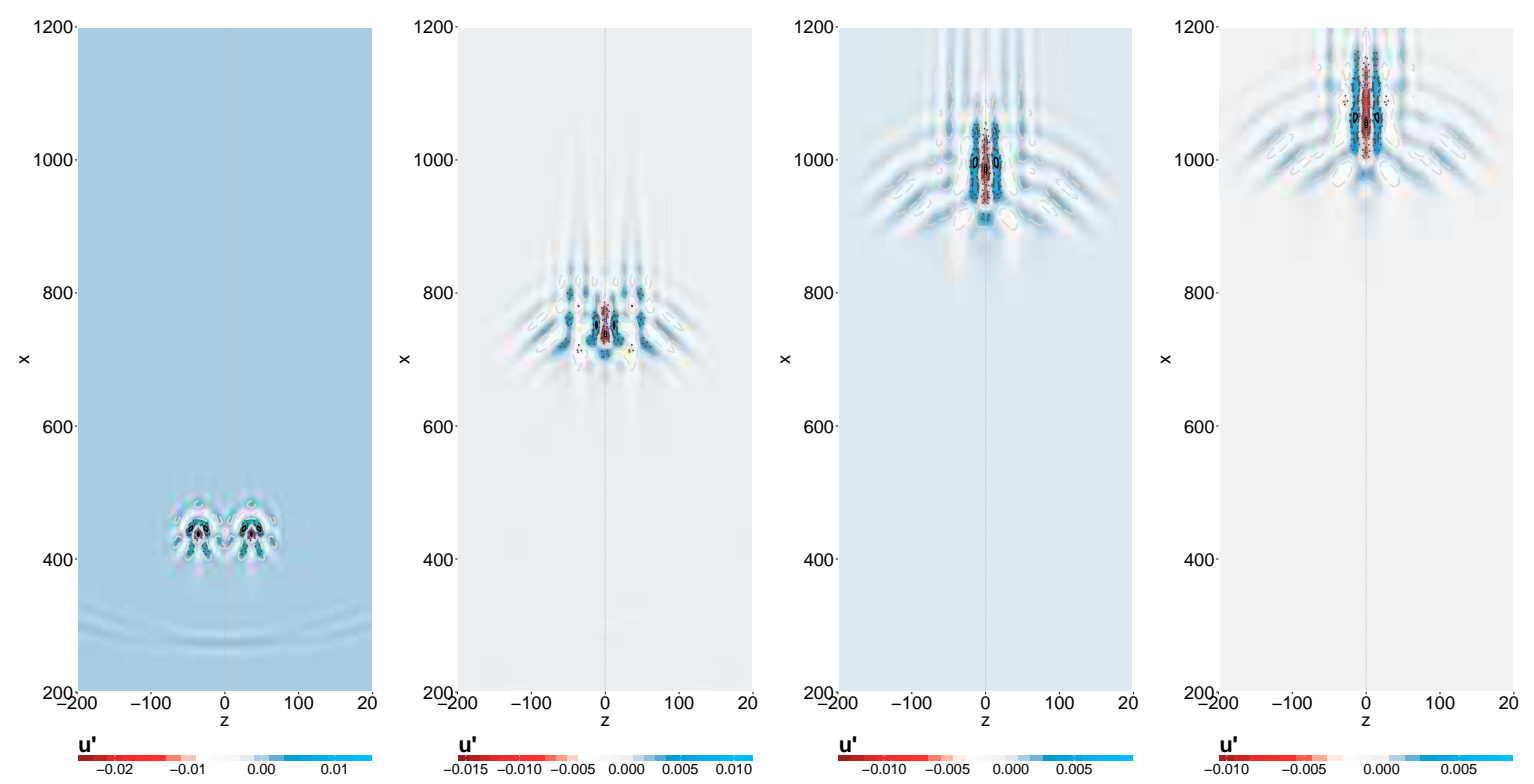

Figure 5.23: Nonlinear evolution in physical space of a pair of wave packets separated by $d=30$. 

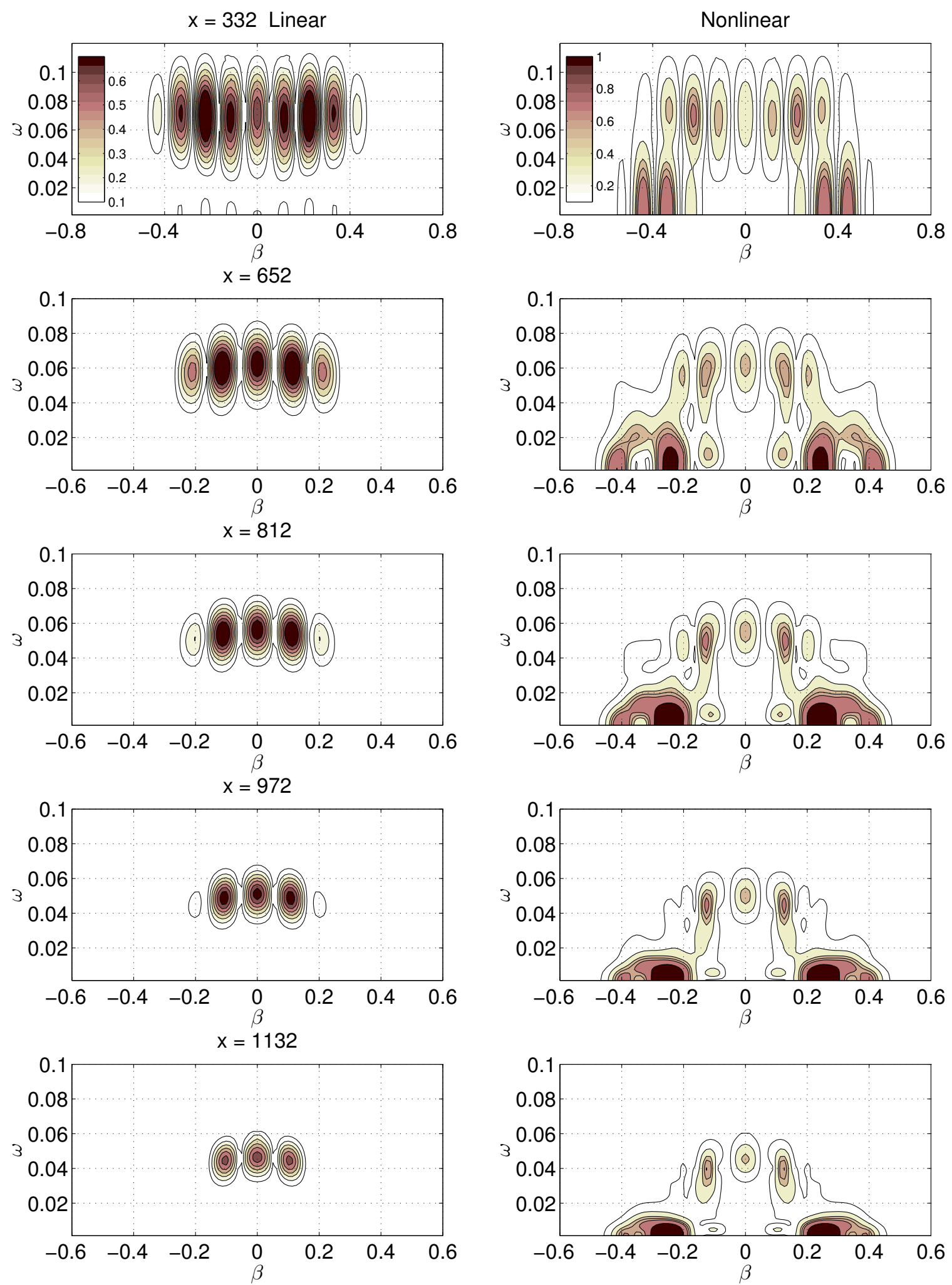

Figure 5.24: Comparison of linear and nonlinear spectral evolution for two packets separated $d=30$. 

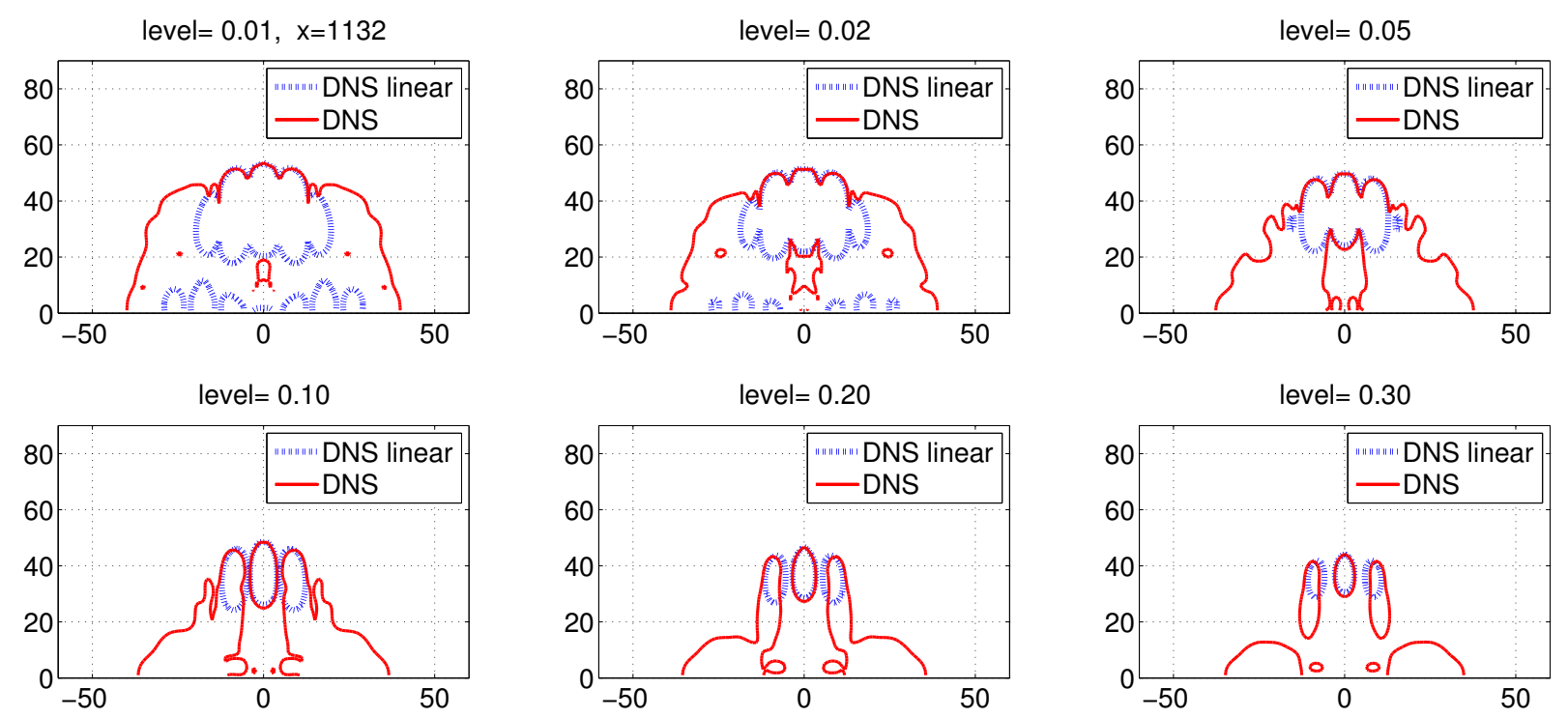

Figure 5.25: Comparison of spectrum contour levels at position $x=1132$.
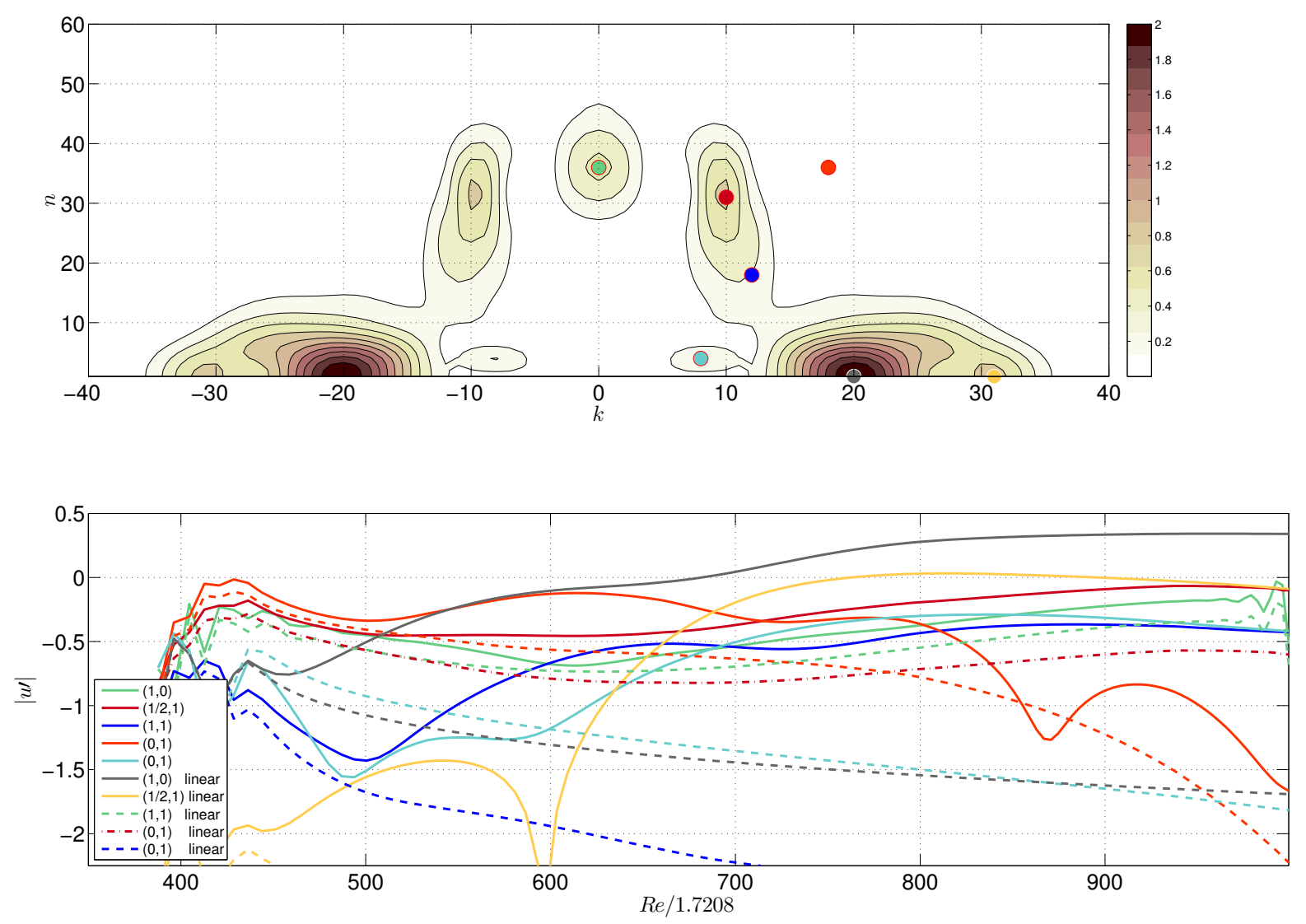

Figure 5.26: Amplification curves for a pair of wave packets separated 30 units. 


\subsubsection{Pair of wave packets separated by $d=60$}

In physical space, the evolution for the linear and nonlinear pair of wave packets are shown in figures 5.27, 5.28 respectively. At the initial positions the two packets can be easily identified, they preserve their individual structure for a substantial part of the evolution. The nonlinear evolution in the last frame in figure 5.28, resembles two isolated packets with a small interaction region at the center of the domain. The steady streaks have similar amplitudes than the isolated packet. The overlap region, presents higher amplitude, but lower than in the separation $d=30$. For better comparison in the physical space, the three cases considered, isolated packet and the two pair interaction, are shown in figure 5.29 .

The spectral evolution for linear and nonlinear cases (figure 5.30) shows amplification for bands centered around specific modes. In nonlinear the case, strong steady streaks are amplified.

The mode $(\omega=0.045, \beta=0.06)$, could be generating the harmonic $(0,0.12)$, that corresponds to amplification of the mode, $(0,2 \beta)$. Also amplification of the mode $(0,4 \beta)$ is observed, as occurs in the oblique transition. There is also a modification of the dominant stationary spanwise mode, in relation to the previous case.

From comparison of spectrum levels at the nonlinear position $x=1132$, linear and nonlinear bands can be easily identified (figure 5.31). The amplification curves were calculated for selected modes (figure 5.32) in several bands. Steady streaks display sustained growth downstream. In general, it is observed a destabilizing effect by the pair of wave packet interaction. However the effect is weaker than observed in closer pair of packets but stronger than for the isolated packet.

In the cases considered, the interaction of wave packets generated regions in the physical space of constructive superposition. This local interaction had a global impact in the spectral evolution, changing drastically the character of the nonlinear evolution in several bands in the spectrum, in comparison with the isolated wave packet. 

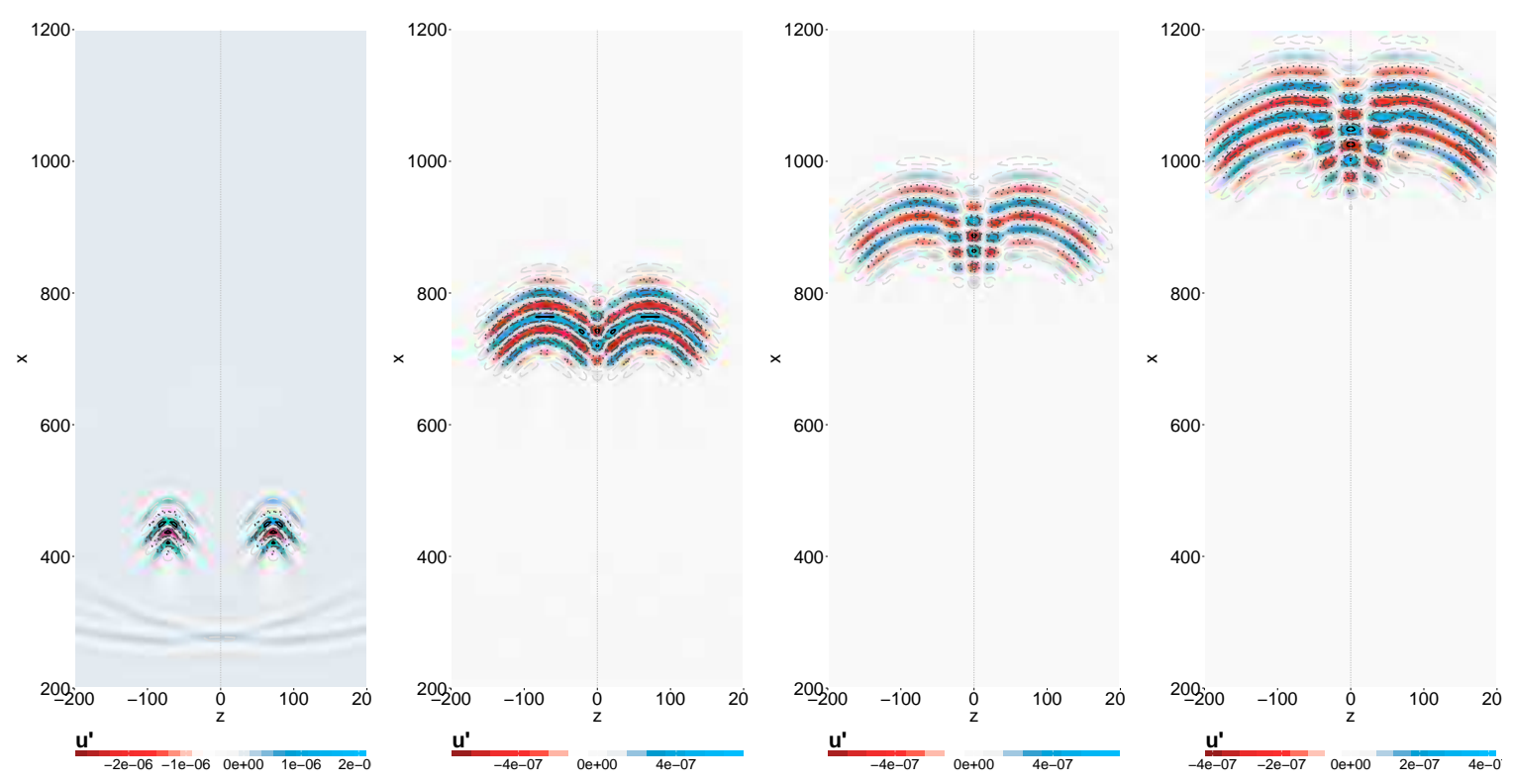

Figure 5.27: Linear evolution of a pair of wave packets separated by $d=60$.
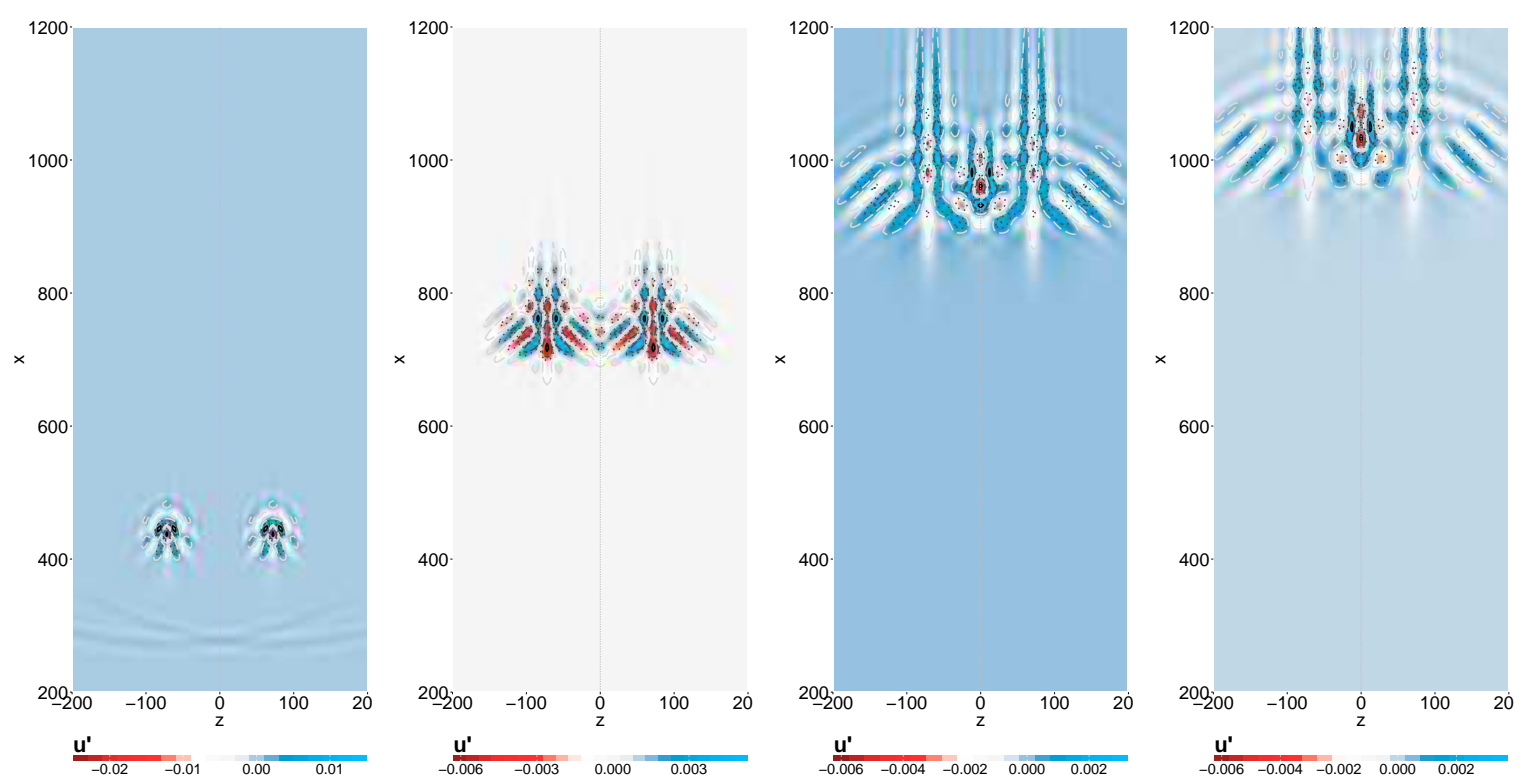

Figure 5.28: Nonlinear evolution of a pair of wave packets separated by $d=60$. 
(a)

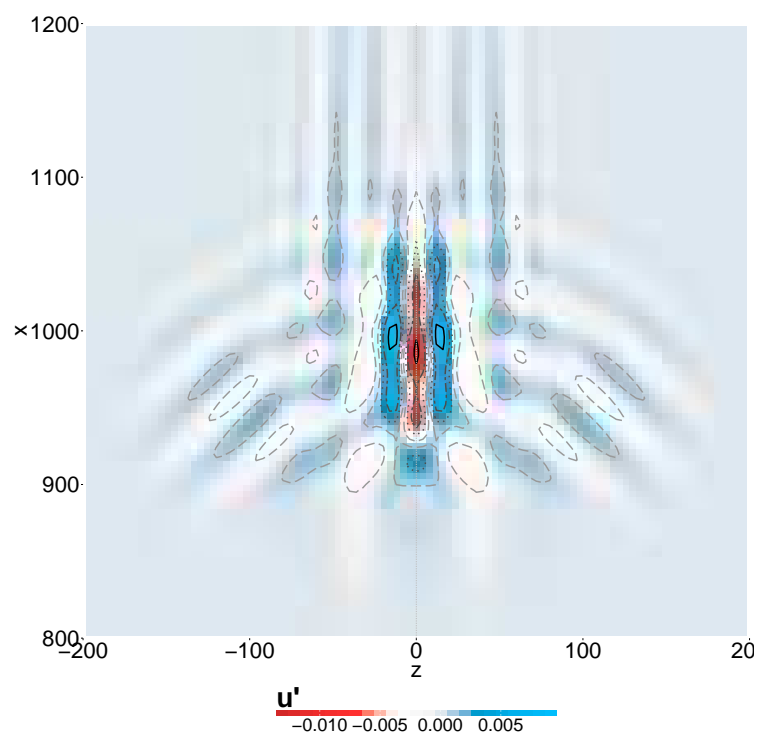

(b)

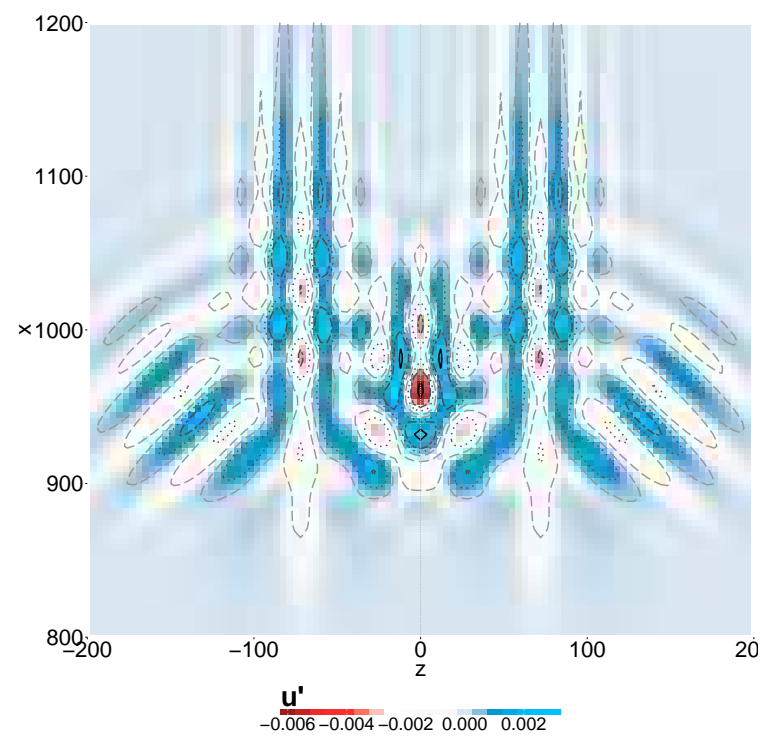

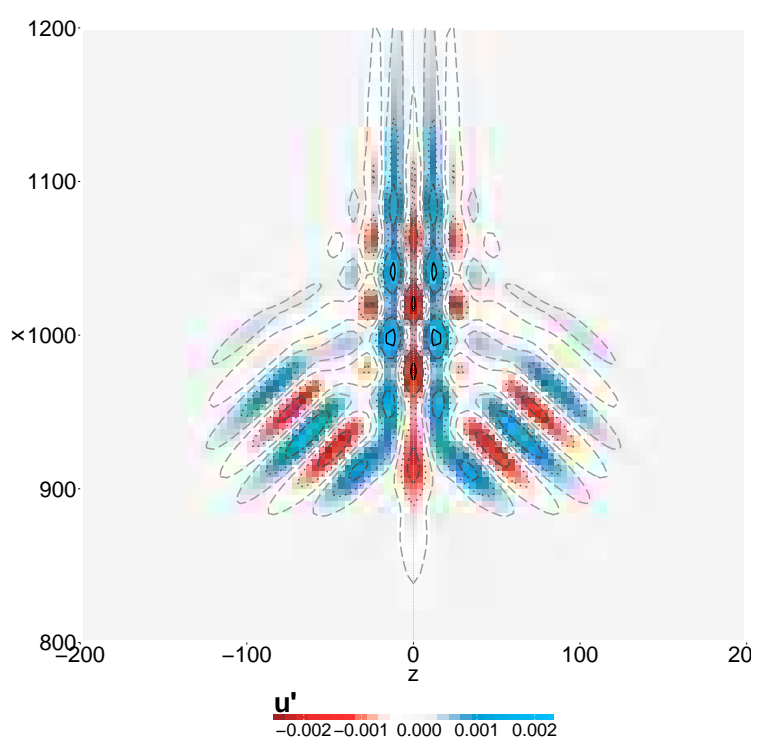

(c)

Figure 5.29: Wave packet interaction, with distances between center packet (a) $d=30$, (b) $d=60$, (c) isolated packet. 

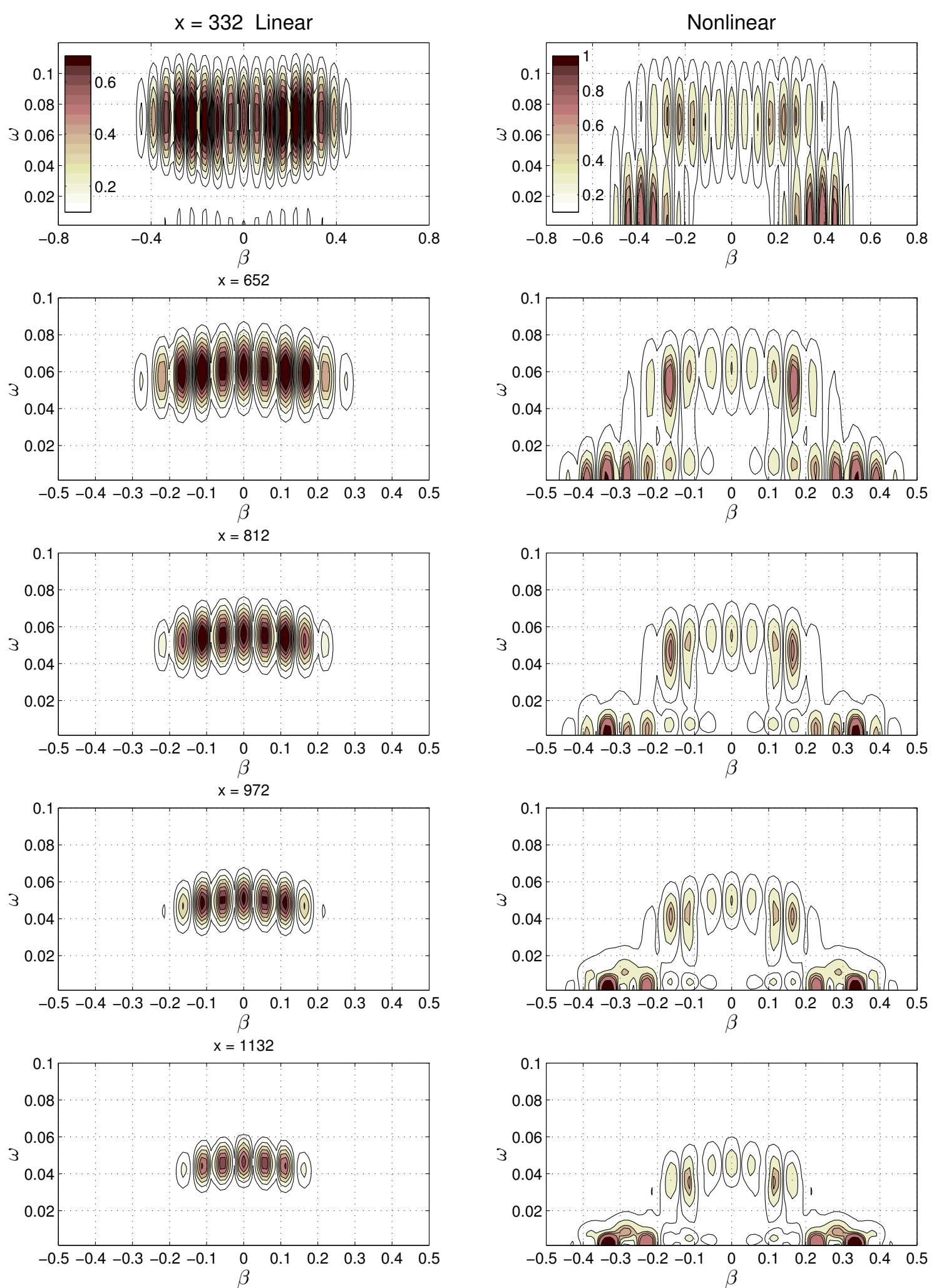

Figure 5.30: Linear and nonlinear spectral evolution for a pair of wave packets separated $d=60$. 

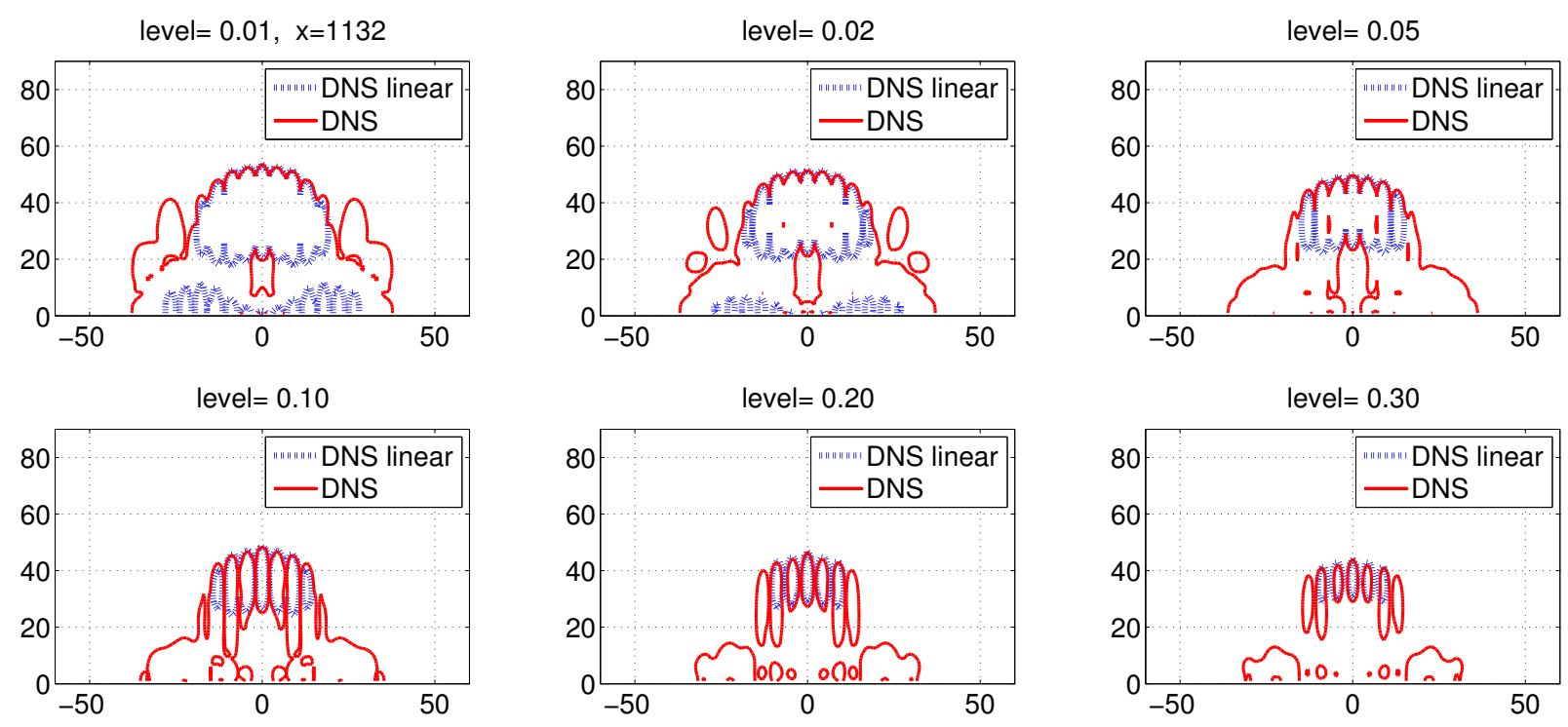

Figure 5.31: Comparison of spectrum levels for a pair of linear and nonlinear wave packets separated $d=60$ at $\mathrm{x}=1132$.
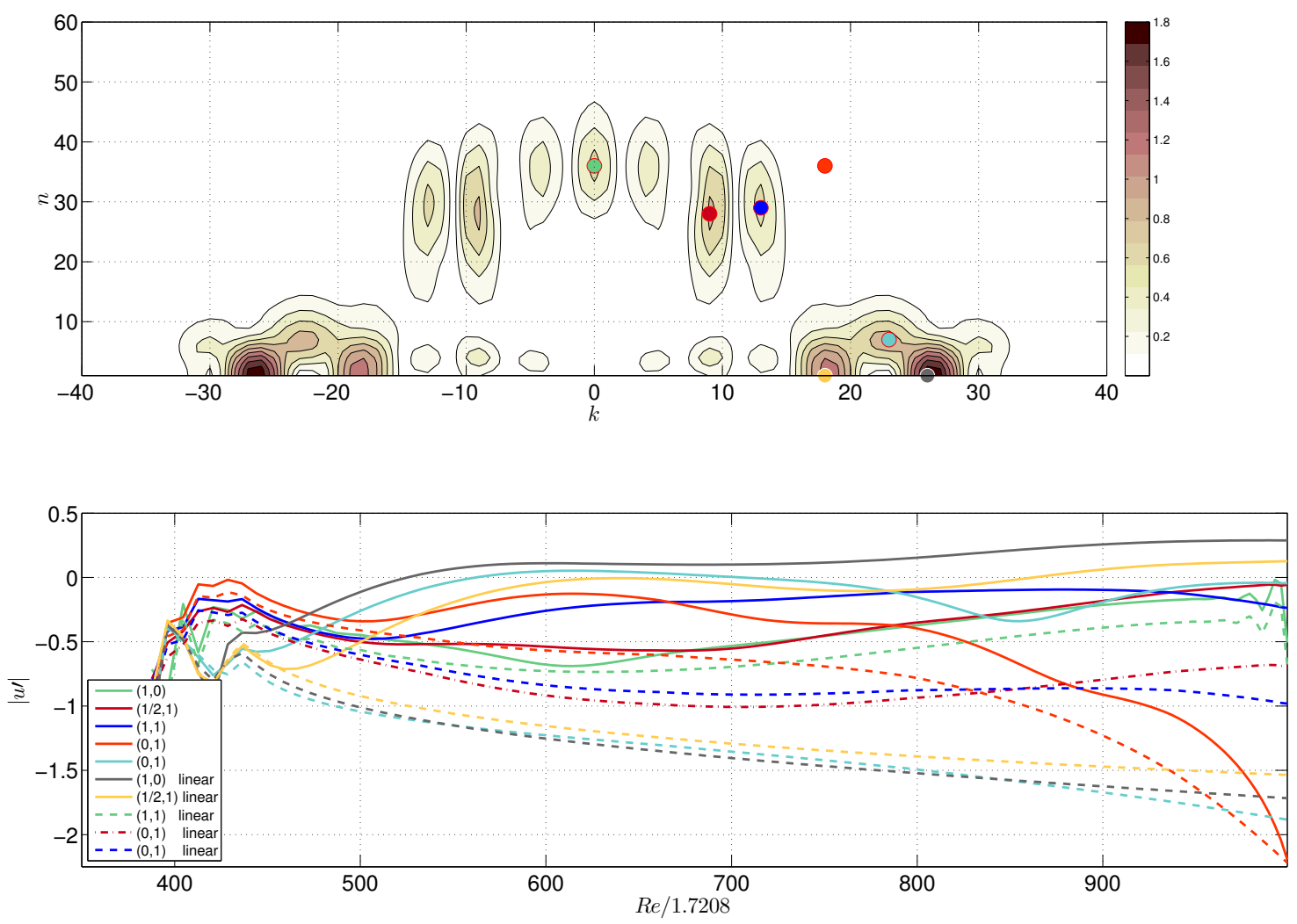

Figure 5.32: Amplification curves for a pair of wave packets separated $d=60$. 


\section{Transition generated by white noise}

A more realistic and hence, complicated scenario for natural transition, is transition generated by white noise. In this chapter results of DNS simulations at Mach 0.2 and Mach 0.9 are presented. In both cases, a low amplitude case (linear) to be used as reference and a nonlinear evolution are considered. Also a preliminary analysis is presented. The focus of this chapter is to evidence the main differences and the influence of compressibility on this kind of problem, to plan future detailed works. Sivasubramanian e Fasel (2014), Sivasubramanian e Fasel (2015) investigated a link between secondary instability mechanisms, wave packet evolution and natural transition for Mach 6. At subsonic Mach numbers there are no works with this approach.

The objective of these simulations is to identify main characteristics of transition induced by white noise and to establish the differences with the wave packet evolution. This information could help to plan future detailed analysis.

\subsection{Generation of white noise disturbance}

A time periodic random signal was generated, with period $T=2 \pi / \omega_{0}$. The random phase $\phi_{k}$ was applied in frequency and spanwise modes, keeping the energy equal for all modes, as can be seen in perturbation spectrum (figure 6.1). The perturbation function employed was:

$$
v^{\prime}=A_{3 D} \sum_{n=1}^{N_{n}} \sum_{k=-N_{k}}^{N_{k}}\left[\cos \left(\alpha_{0}\left(x-x_{0}\right) \pm\left(k \beta_{0} z+\phi_{k}\right)\right) \cos \left(n \omega_{0} t+\phi_{n}\right)\right]
$$

where $-\pi \leq \phi_{n}<\pi$ was generated by using Fortran's rand function. For white noise simulations, the computational domain and flow parameters used in Chapter 5 were maintained. 

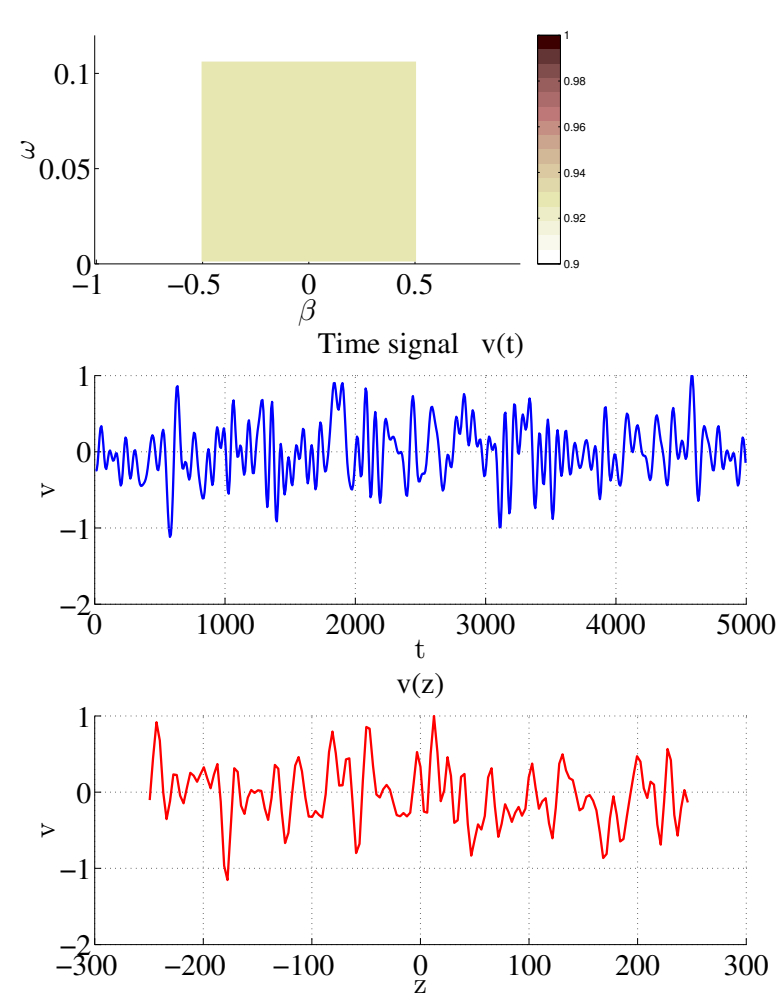
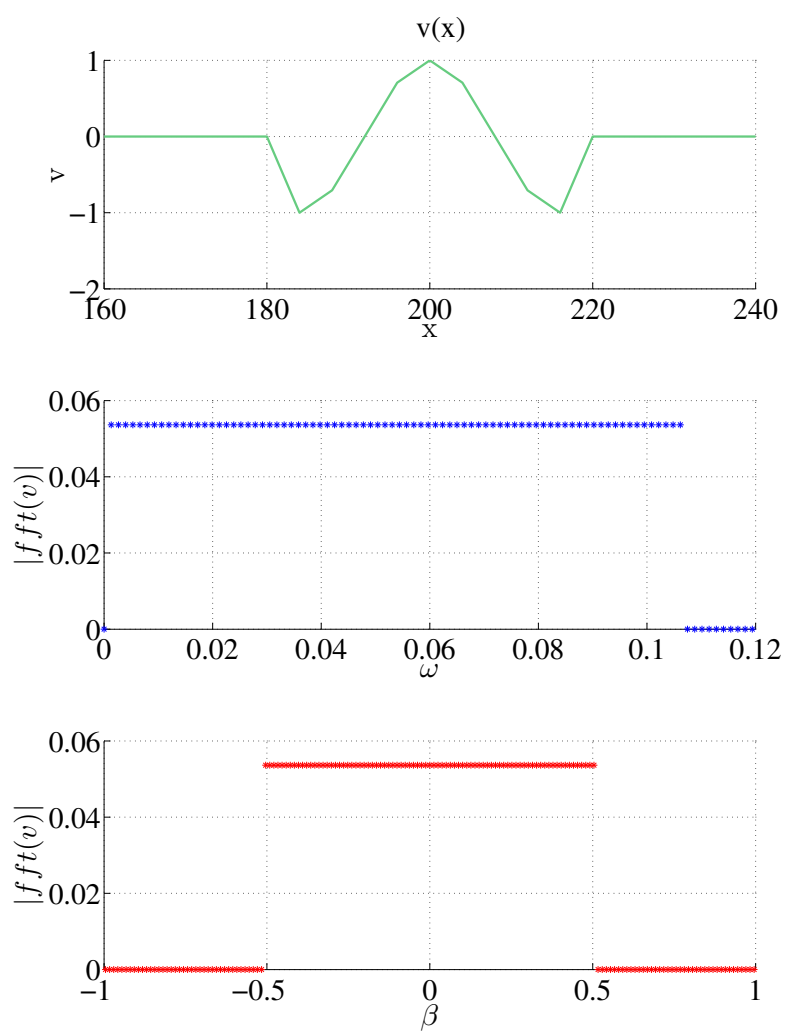

Figure 6.1: Temporal and spatial disturbances used to generate white noise perturbation.

\subsection{White noise evolution at Mach 0.2}

In figure 6.2 linear and nonlinear evolution of white noise are compared in the physical space. Linear case presents smooth distribution of amplitude across entire domain with low amplitude variations, of the order of $\sim 10^{-5}$. The amplitude of the nonlinear case was chosen to provide a linear region after perturbation region, but also to induce nonlinear growth at some downstream position. To observe nonlinear amplification, a disturbance amplitude $\sim 4$ times larger than used for isolated wave packet was needed.

In the nonlinear case, lambda vortex-like structures are observed at localized regions, which have the higher amplitudes. This observation suggest the local presence of fundamental and/or subharmonic resonance. These vortices structures appear and disappear in time. After the initial transient travels out from the computational domain, Fourier analysis was applied in spanwise direction and in time. The spectrum evolution for the linear and the nonlinear cases, is shown in figure (6.4). The linear evolution is identical to that of isolated packet, because the linear evolution is independent of the phase, and the modes do not interact. In the nonlinear evolution linear band remains strong, but now, low frequency modes are amplified (figure 6.5). The linear and the nonlinear bands can be identified in figure 6.6, from the superposition of spectrum levels. 


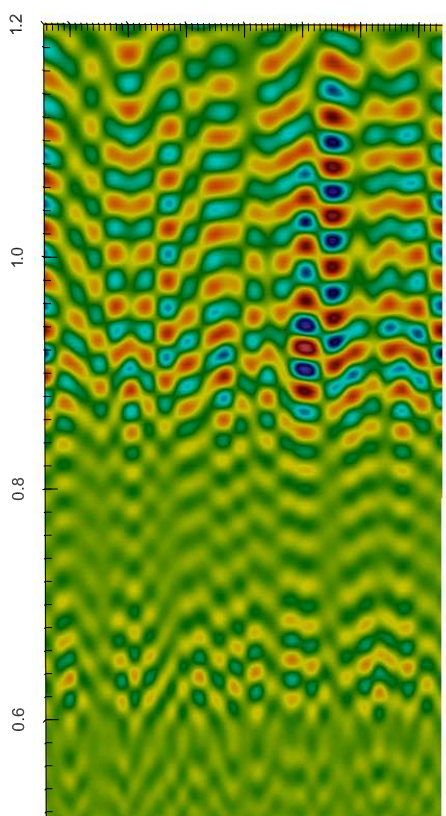

(a)

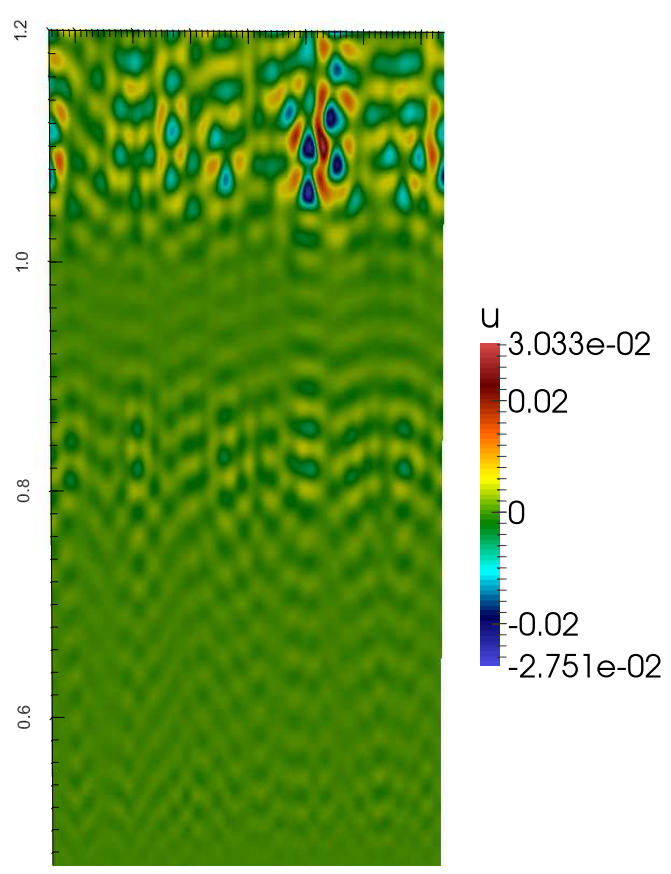

(b)

Figure 6.2: (a) Linear and (b) nonlinear evolution at Mach 0.2 for white noise disturbance. Lambda vortex are generated in nonlinear case.

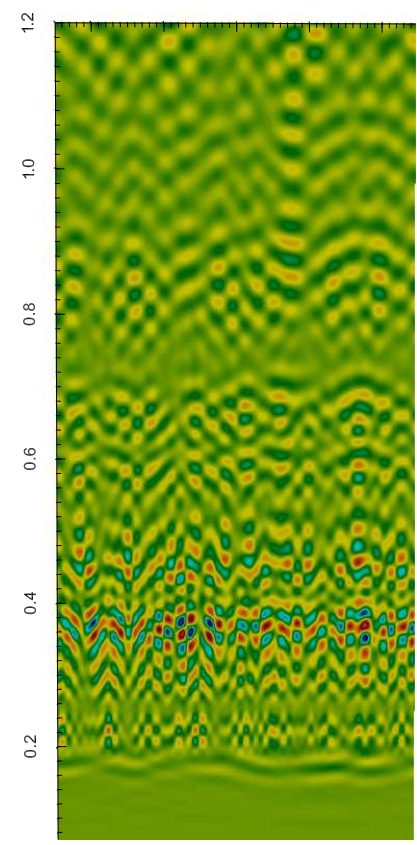

(a)

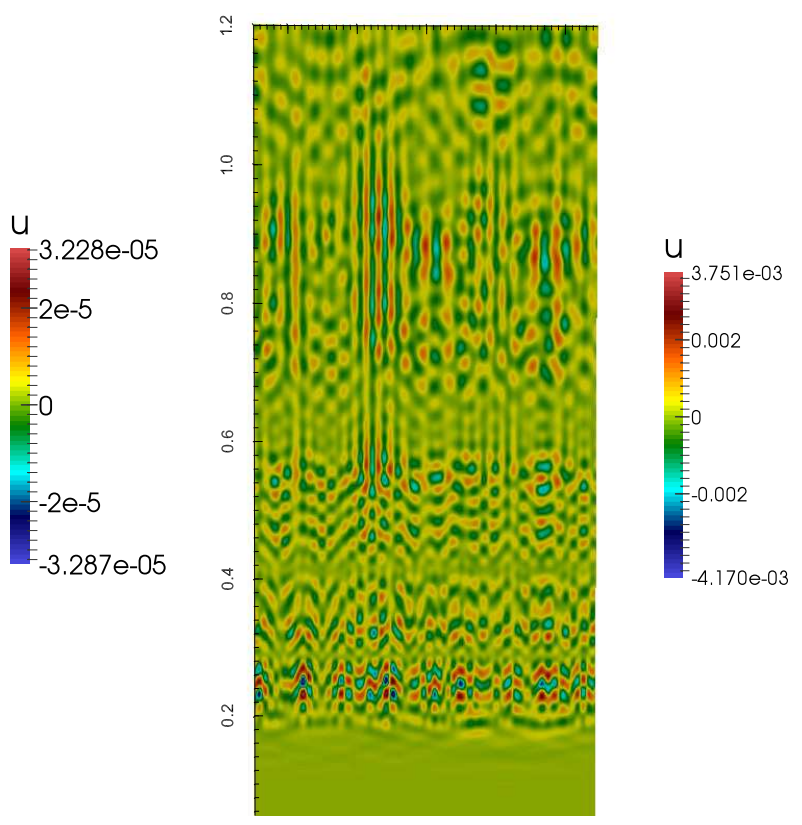

(b)

Figure 6.3: (a) Linear and (b) nonlinear evolution of white noise at Mach 0.9. Streaks along entire domain are generated. 

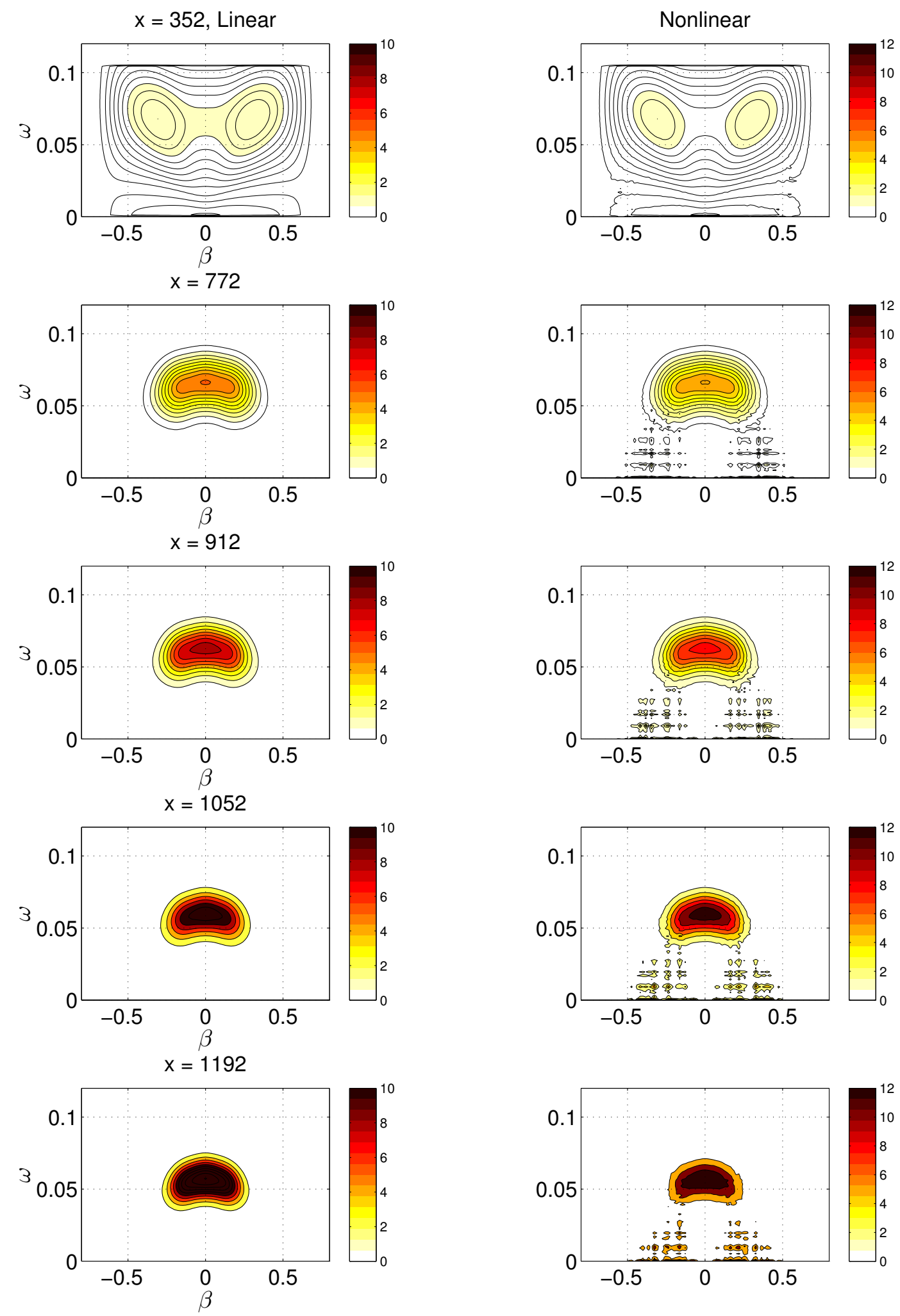

Figure 6.4: Linear and nonlinear evolution of spectral content for white noise perturbation at Mach 0.2 . 


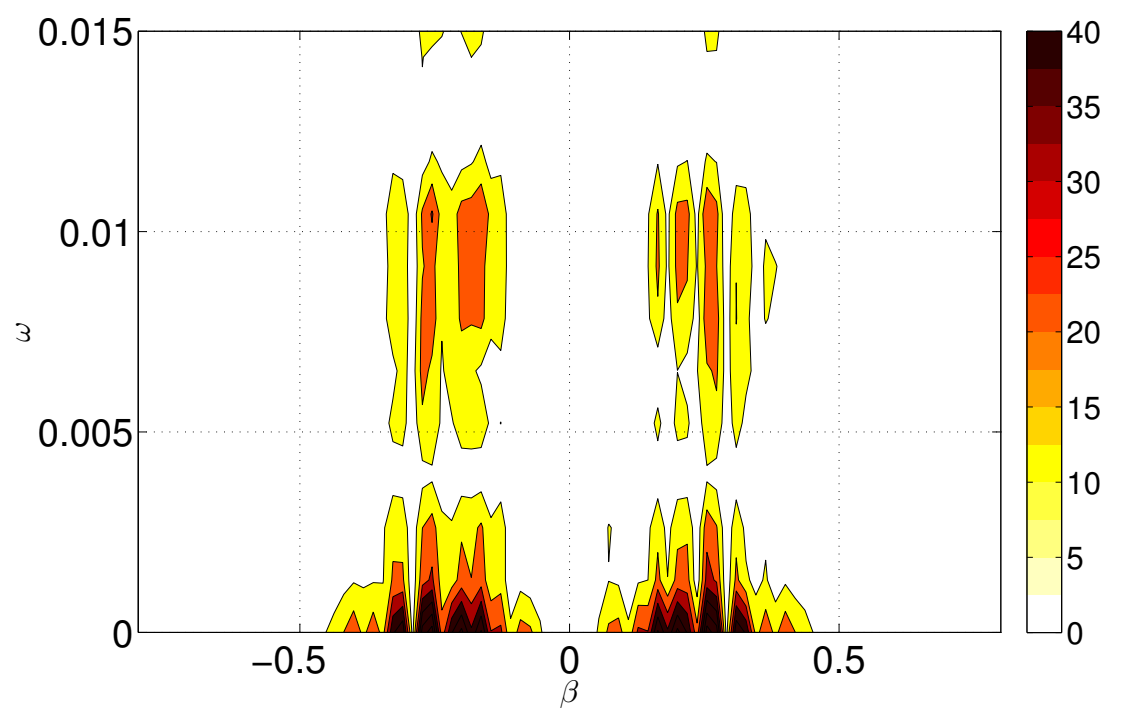

Figure 6.5: Low frequency modes (most amplified) in white noise transition at Mach 0.2.
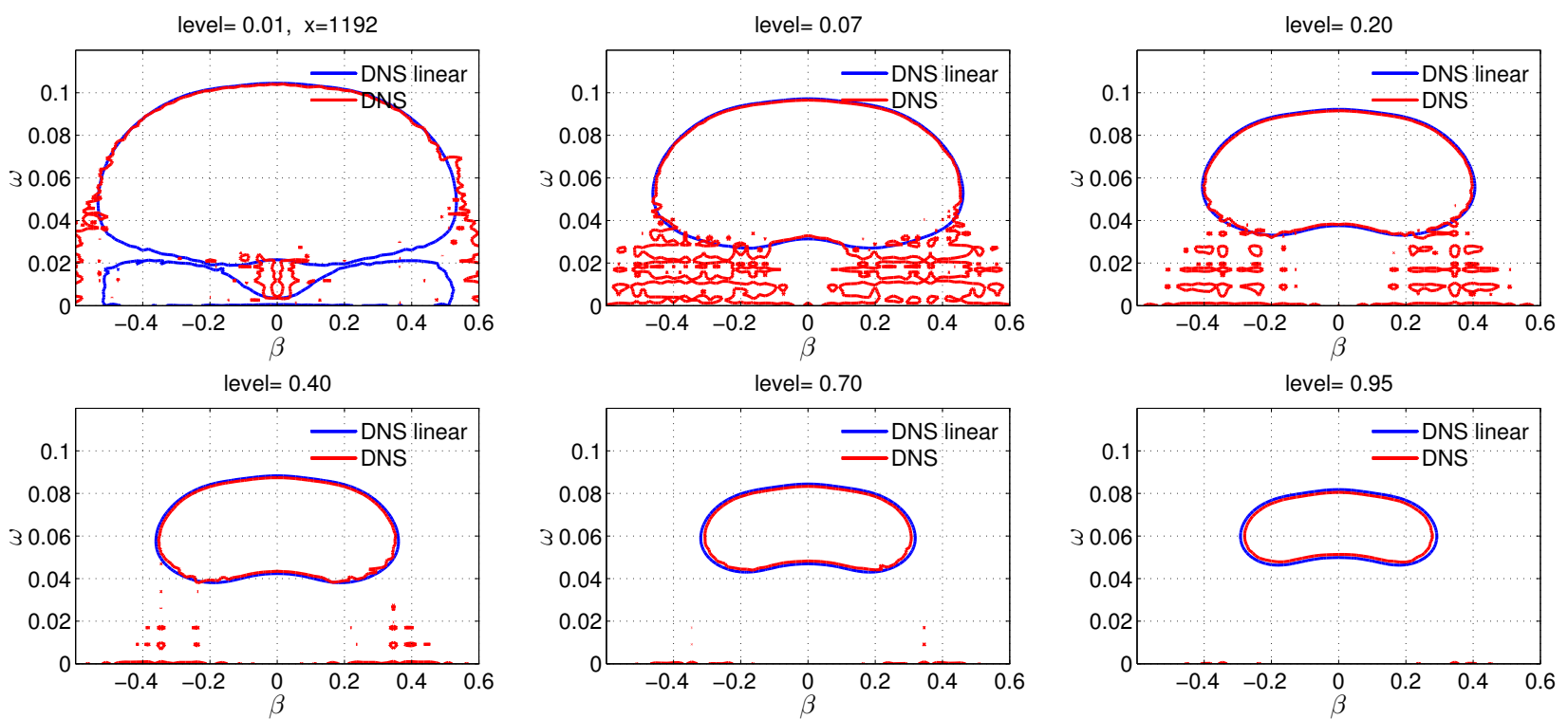

Figure 6.6: Comparison of spectral levels at $\mathrm{x}=1192$. Low frequency modes are amplified nonlinearly. 


\subsection{White noise evolution at Mach 0.9}

In figure 6.3 the linear and nonlinear evolution are compared in the physical space at Mach 0.9. Linear case presents a smooth distribution of amplitude across the entire domain with low amplitude variations, about $\sim 10^{-5}$. The amplitude of the nonlinear case was chosen to provide a linear region downstream of the perturbation region, but also to induce nonlinear growth at some downstream position. To observe nonlinear amplification, a disturbance amplitude $\sim 4$ times larger than used for isolated wave packet was needed.

The nonlinear case result is totally different from the incompressible white noise evolution. Now, from a position around $x \sim 0.6$, longitudinal streaks are observed along the entire domain, that could be linked to oblique transition. After initial transient travels out from the computational domain, Fourier analysis was applied in spanwise direction and time. The spectrum evolution for linear and nonlinear case, is shown in figure (6.7). The linear evolution is identical to an isolated packet, because linear regime is independent of the phase, and the modes do not interact. In the nonlinear evolution, the linear band, is now very weak, in contrast to the incompressible case. Low frequency modes are amplified strongly (figure 6.8). The linear and nonlinear bands can be identified separately by superposition of the spectrum levels (see figure 6.9).

A preliminary localized analysis was performed, by applying a Gaussian windowing in the time velocity signal at a fixed point in space, as shown in figure 6.10(a). From the Fourier transform applied to the windowed signal, low frequency modes have the highest amplitudes (figure 6.10(b)). Also, in this figure, the format of a wave packet can be observed.

A very interesting aspect to note here, is that the wave packet interaction seems to reproduce better aspects of the white noise transition, because in the nonlinear pair packet interaction, low frequency modes were dominant. A more realistic model for natural transition could be constructed considering wave packet interaction, justified by the previous observations. 

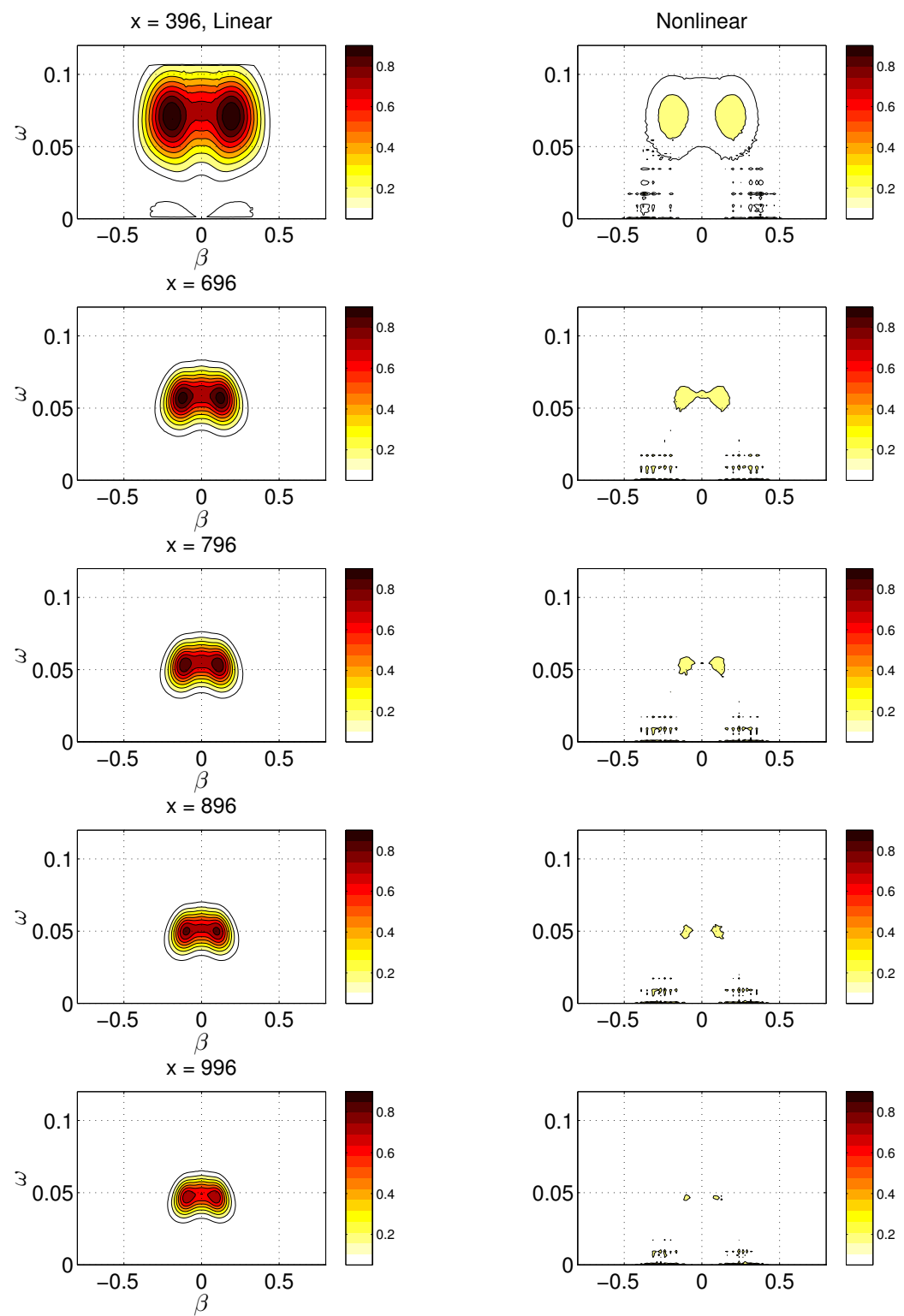

Figure 6.7: Evolution of a white noise perturbation at Mach 0.9.

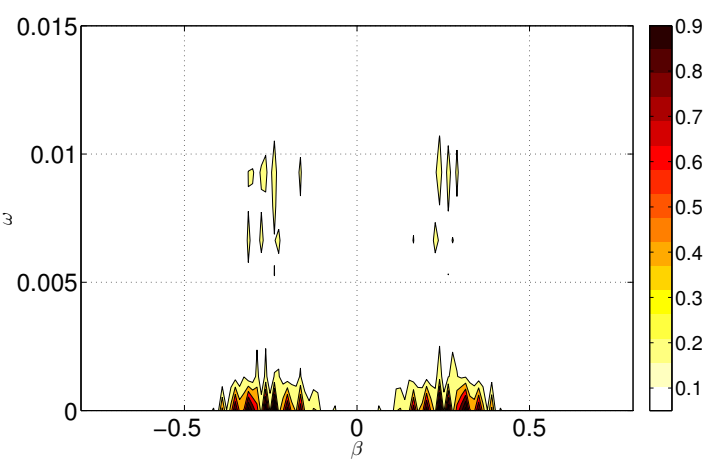

Figure 6.8: Most amplified modes in nonlinear amplification for white noise perturbation at Mach 0.9. 

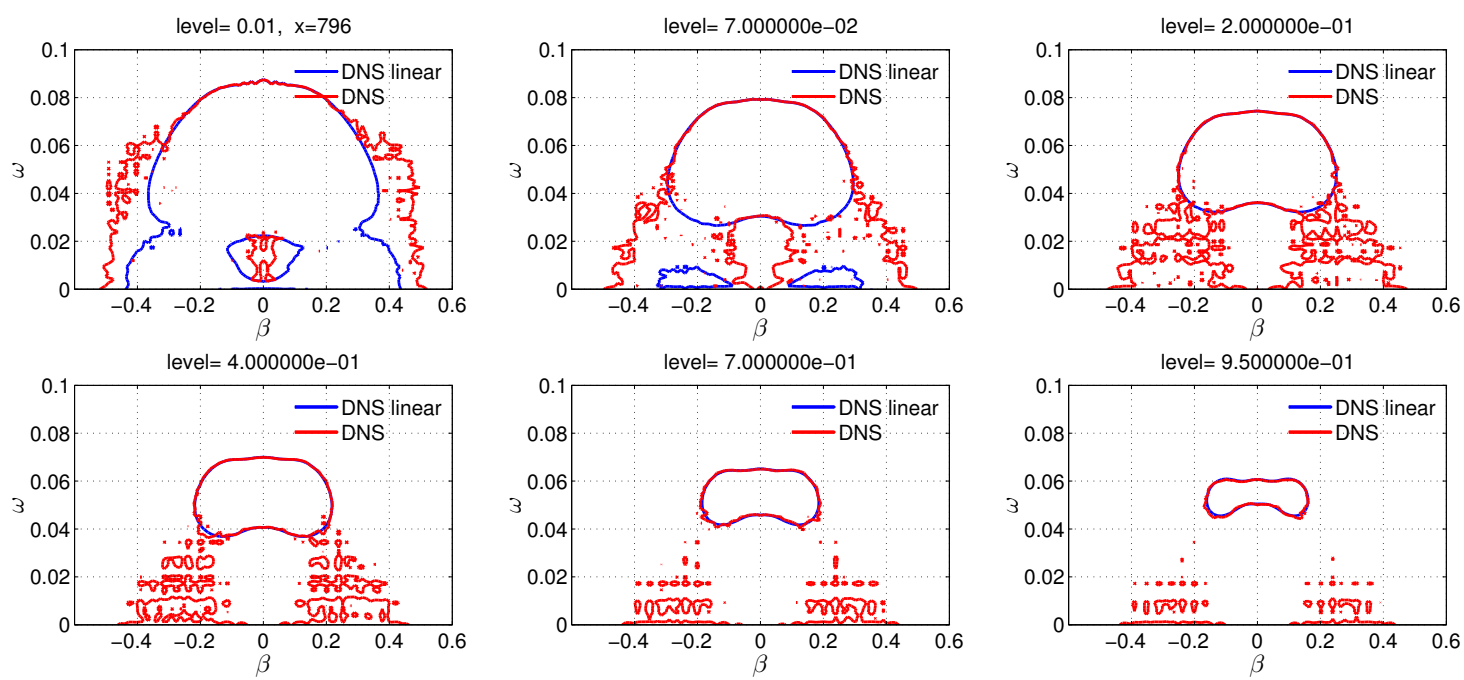

Figure 6.9: Comparison of spectral levels at $\mathrm{x}=796$.

(a)
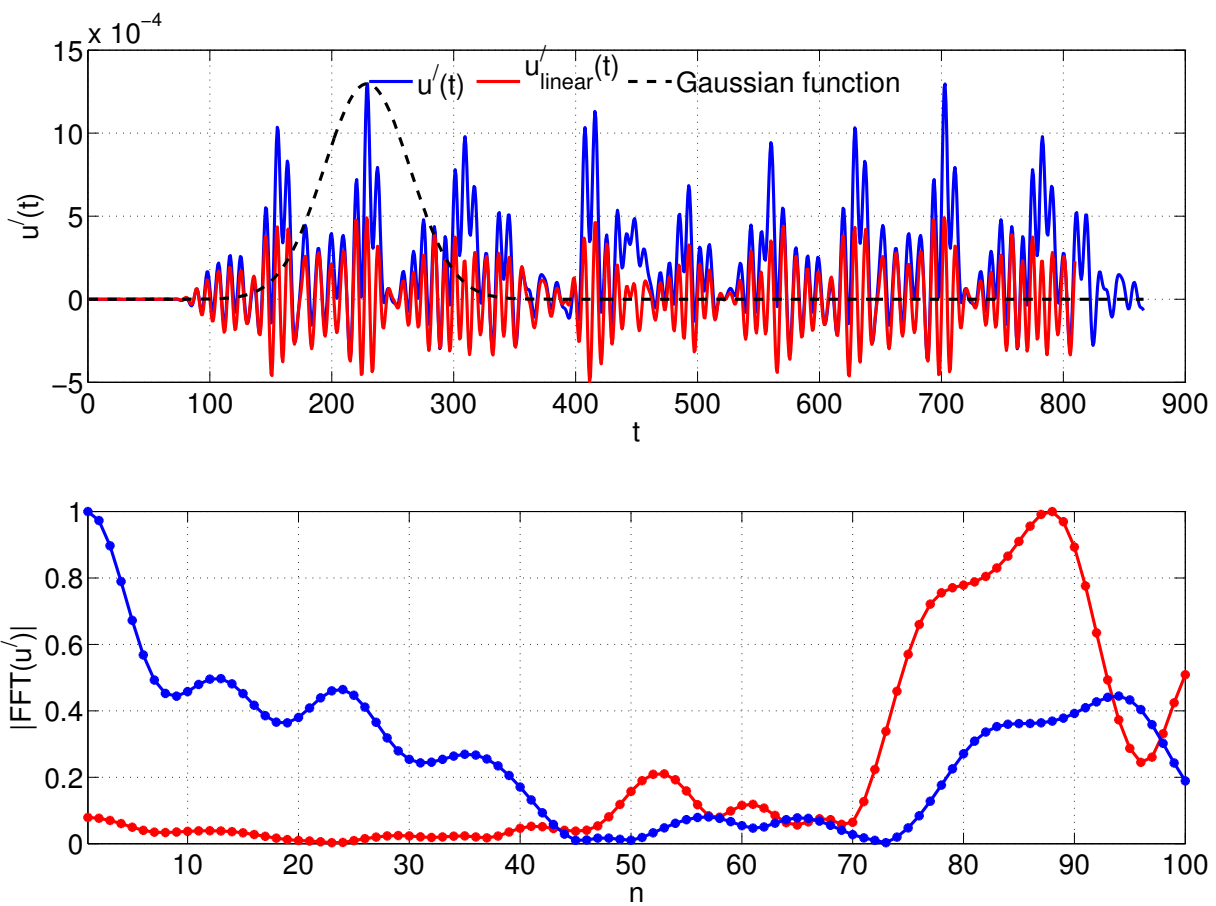

(b)

Figure 6.10: (a) Velocity time signal and Gaussian windowing (b) Fourier transform 


\section{Conclusions and remarks}

\subsection{Conclusions}

A DNS code was developed, to investigate on transition in compressible subsonic boundary layers over a flat plate. Code validation tests were performed, on linear and nonlinear stages of transition, on incompressible and compressible regimes. The focus of the work is to investigate natural transition in subsonic boundary layer, modeled by wave packets and perform a preliminary study of transition induced by white noise. Three main problems were considered, numerical simulation of the experiment (MEDEIROS; GASTER, 1999b) on incompressible boundary layer, the influence of compressibility on wave packet evolution at subsonic Mach numbers and finally, a preliminary study of transition induced by white noise in a boundary layer at Mach 0.2 and 0.9 .

Comparison between numerical and experimental results (MEDEIROS; GASTER, 1999b) are in very good agreement in the linear and nonlinear stages, in both, spatial and Fourier spaces. A nonlinear modal analysis was performed in two bands, subharmonic and fundamental. A numerical investigation of this experiment and a detailed analysis of this kind is not found in the literature for wave packets in the incompressible boundary layer. In the subharmonic band, nonlinear amplification was compared with the controlled case of H-type breakdown, several variables, such as, phase velocity, streamwise wave number, of selected modes were calculated. The growth rates, phase locking and threshold amplitude of the 2D wave of selected modes in the wave packet, corresponds with this mechanism. Sometimes, in literature the modes that could present subharmonic resonance are identified by their peaks in the spectrum in a fixed position. This fact incorrectly suggest, the presence of detuned subharmonic resonance. However, from the analysis performed in Chapter 4, it was concluded that the peaks of the fundamental and subharmonic waves occur at different streamwise positions.

To investigate the origin of the subharmonic modes, they were removed from the pertur- 
bation spectrum. The resulting wave packet from this disturbance, develops an almost identical subharmonic evolution as in previous case, hence, subharmonic modes are generated by previous nonlinear interaction.

In the fundamental band two groups of modes were chosen, with the same spanwise wave number in a range of frequencies. As reported by (COHEN, 1994), both bands have strong nonlinear amplification. The effective amplitude of the 2D mode in the case of controlled transition is of the order of the amplitude of the wave packet. Also, phase locking process is observed between the primary and secondary waves, evidencing the presence of K-type and H-type breakdown. Comparison of the amplification curves for modes with high spanwise wave numbers in the experimental domain, shows good agreement in the growth rates, but the intensity level is underestimated in the the DNS simulation. To attempt to determine the cause of this discrepancy, in the DNS simulation two effective experimental conditions were included. The experimental pressure gradient and experimental asymmetry, last one, added by performing a calibration on each mode of an experimental linear position, in the Fourier space.

Results of these simulations show no influence of these factors on the intensity levels of the modes in the fundamental band. Finally the disturbance amplitude was increased by $20 \%$ to study amplitude effect on the wave packet. In relation with the previous case, differences were found in the fundamental modes, which have higher levels, similar to the observed experimentally, however subharmonic growth rates were overestimated.

Influence of compressibility on the wave packet evolution was investigated in boundary layer at Mach 0.7 and Mach 0.9. In the linear regime, in both cases lower growth rates, than in the incompressible boundary layer were observed. DNS simulations show that linear growth rates at Mach 0.9 are 10 times lower than for Mach 0.2. The frequency of the most linearly unstable 2D mode, decays with Mach number. For Mach $\leq 0.7$ the most linear unstable mode is two-dimensional, at higher Mach numbers the oblique mode are the most linearly unstable, as expected by the results reported in the literature.

At Mach 0.7, two disturbance amplitude were considered, at the highest amplitude, all the selected modes in separated bands have nonlinear amplification. With the lower disturbance amplitude, only modes in the subharmonic band have nonlinear growth. Results suggest that nonlinear mechanisms triggered in the transition process, depends on the disturbance amplitude. The wave packet at Mach 0.9 shows a more stable character, and only has nonlinear amplification in the subharmonic bands. No higher amplitude could be considered because the resultant wave packet become nonlinear from the source.

To represent a possibly closer scenario to natural transition, interaction of a pair of wave packets were considered at Mach 0.9. This value of Mach number was chosen to be typical of transport aircraft at cruise. For the wave packet interaction, two pairs of wave packets were simulated, with different relative distance between them in spanwise direction.

In contrast to the isolated wave packet case, both pair of packets have strong nonlinear 
amplification in several bands. The stationary modes are nonlinearly amplified, which suggest the presence of oblique resonance. Closer packets have a strong nonlinear behavior than the more distant packets. In general, it was found that packet interaction has a stronger nonlinear character than isolated wave packet.

In the cases considered, the interaction of the wave packets generates regions in the physical space of constructive superposition. This local interaction have a global impact in the spectrum evolution, changing drastically the character of the nonlinear evolution in several bands in the spectrum, in relation to the isolated wave packet.

Finally, the transition induced by a white noise disturbance, produced by phase randomization was studied. In the linear regime the spectral evolution is identical to the linear wave packet, as expected, because the phase has no influence in this regime. The nonlinear results for Mach 0.2 and Mach 0.9 are completely different. In the incompressible boundary layer localized lambda vortex structures are observed, that could be associated to the local presence of H-type and/or K-type resonance. In the compressible boundary layer, from a position in downstream, longitudinal vortex are generated, which are distributed across the entire domain. By comparison of the results at Mach 0.9, for isolated wave packet, the packet pair interaction and the transition generated by white noise , the wave packet interaction seems to be a better representation of white noise transition, because has an destabilizing effect and develops longitudinal vortex, as occurs for the the transition induced by white noise. However, a localized analysis is needed to investigate in detail this kind of transition. As a general conclusion in the white noise transition, compressibility has a stronger effect that in the wave packet evolution.

\section{Suggested work}

In the wave packet evolution there are several parameters that deserve a systematic investigation, to identify separately their role in the transition process. From the results of the present work, some factors seem to be interesting, such as the disturbance amplitude and disturbance spectra. It is known that the wall temperature has an important influence on the stability of the compressible boundary layer. At subsonic Mach numbers there are no works of wave packets for this regime. In Chapter 6, preliminary results for transition induced by white noise were presented, a deeper investigation can be done for this problem, considering several random perturbations and applying local analysis techniques. Also, the impact of low level white noise disturbance on the wave packet evolution may help to link features of nonlinear stages observed in wave packet with natural transition. 


\section{Bibliography}

2DECOMP\&FFT. http://www.2decomp.org/.

ANDERSON, J. Hypersonic and High Temperature Gas Dynamics. [S.l.]: AIAA Education, 1989.

BAKE, S.; FERNHOLZ, H. H.; KACHANOV, Y. S. Resemblance of k- and n-regimes of boundary-layer transition at late stages. European Journal of Mechanics - B/Fluids, v. 19, n. 1, p. 1 - 22, 2000. ISSN 0997-7546. Disponível em: <http://www.sciencedirect.com/science/article/pii/S0997754600001084>.

BECH, K. H.; HENNINGSON, D. S.; HENKES, R. A. W. M. Linear and nonlinear development of localized disturbances in zero and adverse pressure gradient boundary-layers. Physics of Fluids, v. 10, n. 6, 1998.

BERGAMO, L. F. Instabilidade hidrodinamica linear do escoamento compressivel em uma cavidade. Dissertação (Mestrado) — Universidade de São Paulo, Escola de Engenharia de São Carlos, Universidade de São Paulo, 2013.

BERGAMO, L. F. et al. Compressible modes in a square lid-driven cavity. Aerospace Science and Technology, v. 44, p. 125 - 134, 2015. ISSN 1270-9638. Instability and Control of Massively Separated Flows.

BERLIN, S.; WIEGEL, M.; HENNINGSON, D. S. Numerical and experimental investigations of oblique boundary layer transition. Journal of Fluid Mechanics, v. 393, p. 23-57, 8 1999. ISSN 1469-7645. Disponível em: <http://journals.cambridge.org.sci-hub.io/article_S002211209900511X>.

BERTOLOTTI, F. P.; HERBERT, T.; SPALART, P. R. Linear and nonlinear stability of the Blasius boundary layer. J. Fluid Mech., v. 242, p. 441-474, 1992.

BESTEK, H.; THUMM, A.; FASEL, H. Thirteenth international conference on numerical methods in fluid dynamics: Proceedings of the conference held at the consiglio nazionale delle ricerche rome, italy, 6-10 july 1992. In: Berlin, Heidelberg: Springer Berlin Heidelberg, 1993. cap. Direct numerical simulation of the three-dimensional breakdown to turbulence in compressible boundary layers, p. 145-149. ISBN 978-3-540-47551-4. Disponível em: <http://dx.doi.org/10.1007/3-540-56394-6_205>. 
BORODULIN, V. I.; KACHANOV, Y. S.; KOPTSEV, D. B. Experimental study of resonant interactions of instability waves in a self-similar boundary layer with an adverse pressure gradient: I. tuned resonances. Journal of Turbulence, v. 3, p. N62, 2002. Disponível em: <http://dx.doi.org/10.1088/1468-5248/3/1/062>.

BORODULIN, V. I. et al. Experimental study of resonant interactions of instability waves in self-similar boundary layer with an adverse pressure gradient: Ii. detuned resonances. Journal of Turbulence, v. 3, p. N63, 2002. Disponível em: <http://dx.doi.org/10.1088/1468-5248/3/1/063>.

BRANDT, L.; SCHLATTER, P.; HENNINGSON, D. S. Transition in boundary layers subject to free-stream turbulence. Journal of Fluid Mechanics, v. 517, p. 167-198, 9 2004. ISSN 1469-7645. Disponível em: <http://journals.cambridge.org/article_S0022112004000941>.

BREUER, K. S.; COHEN, J.; HARITONIDIS, J. H. The late stages of transition induced by a low-amplitude wavepacket in a laminar boundary layer. J. Fluid Mech., v. 340, p. 395-411, 1997.

CHANG, C.-L. et al. Compressible stability of growing boundary layers using parabolized stability equations. In: [S.l.]: American Institute of Aeronautics and Astronautics, 1991. (Fluid Dynamics and Co-located Conferences). Doi:10.2514/6.1991-1636.

CHRISTIAN, S.; J., K. M.; SIEGFRIED, W. Navier-Stokes Simulation of Harmonic Point Disturbances in an Airfoil Boundary Layer. AIAA Journal, American Institute of Aeronautics and Astronautics, v. 38, n. 8, p. 1369-1376, 2000. ISSN 0001-1452. Doi: $10.2514 / 2.1136$.

COHEN, J. The initial evolution of a wave packet in a boundary layer. Phys. Fluids, v. 6, n. 3, p. 1133-1143, March 1994.

COHEN, J.; BREUER, K. S.; HARITONIDIS, J. H. On the evolution of a wave packet in a laminar boundary layer. J. Fluid Mech., v. 225, p. 575-606, 1991.

CRAIK, A. D. D. A model for subharmonic resonance within wavepackets in unstable boundary layers. J. Fluid Mech., v. 432, p. 409-418, 2001.

CRIMINALE, W. O.; JACKSON, T. L.; JOSLIN, R. D. Theory and Computation in hydrodynamic stability. [S.l.]: Cambridge University Press, 2003.

DENG, S. et al. DNS for K- and H-Type flow transition over a flat plate. 43rd AIAA Aerospace Sciences Meeting and Exhibit, American Institute of Aeronautics and Astronautics, jan. 2005. Doi:10.2514/6.2005-666.

DINI, P.; SELIG, M. S.; MAUGHMER, M. D. Simplified linear stability transition prediction method for separated boundary layers. AIAA journal, v. 30, n. 8, p. 1953-1961, 1992.

El-Hady Nabil M. Spatial three-dimensional secondary instability of compressible boundary-layer flows. AIAA Journal, American Institute of Aeronautics and Astronautics, v. 29, n. 5, p. 688-696, 1991. ISSN 0001-1452. Doi: 10.2514/3.10642.

FASEL, H. Investigation of the stability of boundary layers by a finite-difference model of the Navier-Stokes equations. J. Fluid Mech., v. 78, p. 355-383, 1976. 
. Numerical simulation of nonlinear growth of wave packets in a boundary layer. In: TATSUMI, T. (Ed.). Turbulence and Chaotic Phenomena in Fluids. [S.l.]: Elsevier, 1984.

FASEL, H.; KONZELMANN, U. Non-parallel stability of a flat-plate boundary layer using the complete Navier-Stokes equations. J. Fluid Mech., v. 221, p. 311-347, 1990.

FASEL, H. F. Numerical investigation of the interaction of the klebanoff-0mode with a tollmien-schlichting wave. Journal of Fluid Mechanics, v. 450, p. 1-33, 1 2002. ISSN 1469-7645. Disponível em: <http://journals.cambridge.org/article_S0022112001006140>.

FEDOROV, A. Transition and stability of high-speed boundary layers. Annual Review of Fluid Mechanics, Annual Reviews, v. 43, p. 79-95, 2011.

FORGOSTON, E.; TUMIN, A. Three-dimensional wave packets in a compressible boundary layer. Physics of Fluids, AIP, v. 18, n. 10, 2006.

FRANKO, K. J.; LELE, S. K. Breakdown mechanisms and heat transfer overshoot in hypersonic zero pressure gradient boundary layers. Journal of Fluid Mechanics, v. 730, p. 491-532, 9 2013. ISSN 1469-7645. Disponível em: <http://journals.cambridge.org/article_S0022112013003509>.

GAPONOV, S. A. et al. Development of small perturbations in a slightly nonparallel supersonic flow. Journal of Applied Mechanics and Technical Physics, v. 23, n. 3, p. 398-401, 1982. ISSN 1573-8620. Disponível em: <http://dx.doi.org/10.1007/BF00910082>.

GASTER, M. A theoretical model of a wave packet in the boundary layer on a flat plate.

Proc. R. Soc. London A, v. 347, p. 271-289, 1975.

GASTER, M.; GRANT, I. An experimental investigation of the formation and development of a wavepacket in a laminar boundary layer. Proc. Royal Soc. of London A, v. 347, p. 253-269, 1975.

GASTER, M. a. New approaches and concepts in turbulence. In: Basel:

Birkhäuser Basel, 1993. cap. The Origins of Turbulence, p. 235-250. ISBN 78-3-0348$8585-0$.

INGEN, J. van. The eN Method for Transition Prediction. Historical Review of Work at TU Delft. In: . [S.l.]: American Institute of Aeronautics and Astronautics, 2008. (Fluid Dynamics and Co-located Conferences). Doi:10.2514/6.2008-3830.

JOSLIN, R. D.; STREETT, C. L.; CHANG, C.-L. 1993.

KACHANOV, Y. S. On the resonant nature of the breakdown of a laminar boundary layer. J. Fluid Mech., v. 184, p. 43-74, 1987.

Physical mechanisms of laminar boundary layer transition. Ann. Rev. Fluid Mech., v. 26, p. 411-482, 1994.

KACHANOV, Y. S.; KOZLOV, V. V.; LEVCHENKO, V. Y. Nonlinear development of waves in a boundary layer. Izv. Akad. Nauk SSSR, Mekh. Zhidk. Gaza, v. 3, p. 49-53, 1977. In Russian. 
KACHANOV, Y. S.; LEVCHENKO, V. Y. The resonant interaction of disturbances at laminar-turbulent transition in a boundary layer. J. Fluid Mech., v. 138, p. 209-247, 1984.

KARNIADAKIS, R. M. K. I. G. E. Parallel Scientific Computing in C++ and MPI: A Seamless Approach to Parallel Algorithms and their Implementation. 1st edition. ed. Cambridge University Press, 2003. ISBN 9780521520805,0521520800. Disponível em: <http://gen.lib.rus.ec/book/index.php?md5=891B2CD03344654624C22E04A87B7B53>.

KAYNAK, U.; GüRDAMAR, E. Boundary-Layer Transition Under the Effect of Compressibility for the Correlation Based Transition Model. In: . [S.l.]: American Institute of Aeronautics and Astronautics, 2008. (Aerospace Sciences Meetings). Doi:10.2514/6.2008-774.

KAZAKOV, A. V.; KOGAN, M. N.; KUPAREV, V. A. The stability of the subsonic boundary layer during heating of the surface of a flat plate near the leading edge. Fluid Dynamics, v. 20, n. 3, p. 394-398, 1985. ISSN 1573-8507. Disponível em: <http://dx.doi.org/10.1007/BF01049991>.

KLEBANOFF, P. S.; TIDSTROM, K. D.; SARGENT, L. M. The three-dimensional nature of boundary layer instability. J. Fluid Mech., v. 12, p. 1-34, 1962.

KONZELMANN, U.; FASEL, H. Numerical simulation of a three-dimensional wave packet in a growing flat plate boundary layer. In: THE ROYAL AERONAUTICAL SOCIETY. Boundary layer Transition and Control. Cambridge - UK, 1991. p. 24.1-24.11.

KUROTAKI, T. et al. Numerical Simulation around Airfoil with Natural Transition in High Reynolds Numbers. In: [S.l.]: American Institute of Aeronautics and Astronautics, 2007. (Fluid Dynamics and Co-located Conferences). Doi:10.2514/6.20073841.

\section{LAPACK. http://www.netlib.org/lapack/.}

LELE, S. K. Compact finite difference schemes with spectral-like resolution. J. Comp. Phys., v. 103, p. 16-42, 1992.

LIU, Z. et al. Direct numerical simulation of transition in a subsonic airfoil boundary layer. In: [S.1.]: American Institute of Aeronautics and Astronautics, 1997. (Aerospace Sciences Meetings). Doi:10.2514/6.1997-752.

MACK, L. Linear Stability Theory and the Problem of Supersonic Boundary- Layer Transition. AIAA Journal, American Institute of Aeronautics and Astronautics, v. 13, n. 3, p. 278-289, 1975. ISSN 0001-1452. Doi: 10.2514/3.49693.

MACK, L. M. Stability of time dependent and spatially varying flows: Proceedings of the symposium on the stability of time dependent and spatially varying flows held august 19-23, 1985, at nasa langley research center, hampton, virginia. In: . New York, NY: Springer New York, 1987. cap. Review of Linear Compressible Stability Theory, p. 164-187. ISBN 978-1-4612-4724-1. Disponível em: <http://dx.doi.org/10.1007/978-14612-4724-1_9>. 
MAREC, J.-P. Aerodynamic drag reduction technologies: Proceedings of the ceas/dragnet european drag reduction conference, 19-21 june 2000, potsdam, germany. In: _ Berlin, Heidelberg: Springer Berlin Heidelberg, 2001. cap. Drag Reduction: a Major Task for Research, p. 17-27. ISBN 978-3-540-45359-8. Disponível em: $<$ http://dx.doi.org/10.1007/978-3-540-45359-8_3>.

MARTINEZ, A.; MEDEIROS, M. F. Direct numerical simulation of a wavepacket in a boundary layer at mach 0.9. 46th AIAA Fluid Dynamics Conference, American Institute of Aeronautics and Astronautics, jun 2016. Doi:10.2514/6.2016-3195.

MARTINEZ, A. G.; GENNARO, E. M.; MEDEIROS, M. A. Wavepackets in boundary layers close to transonic speeds. Procedia IUTAM, v. 14, p. $374-380,2015$. ISSN 2210-9838. IUTAM_ABCM Symposium on Laminar Turbulent Transition. Disponível em: <http://www.sciencedirect.com/science/article/pii/S2210983815000899>.

MASAD, J. A.; NAYFEH, A. H. Effect of heat transfer on the subharmonic instability of compressible boundary layers. Physics of Fluids A, v. 3, n. 9, p. 2148-2163, 1991. Disponível em: <http://scitation.aip.org/content/aip/journal/pofa/3/9/10.1063/1$.857897>$.

MAYER, C. S. J.; TERZI, D. A. V.; FASEL, H. F. Direct numerical simulation of complete transition to turbulence via oblique breakdown at mach 3. Journal of Fluid Mechanics, v. 674, p. 5-42, 5 2011. ISSN 1469-7645. Disponível em: <http://journals.cambridge.org/article_S0022112010005094>.

MAYER, C. S. J.; WERNZ, S.; FASEL, H. F. Numerical investigation of the nonlinear transition regime in a mach 2 boundary layer. Journal of Fluid Mechanics, v. 668, p. 113-149, 2 2011. ISSN 1469-7645. Disponível em: <http://journals.cambridge.org/article_S0022112010004556>.

MEDEIROS, M. A. et al. Iutam abcm symposium on laminar turbulent transition nonlinear spanwise modulated waves in channel flow. Procedia IUTAM, v. 14, p. 355 - 363, 2015. ISSN 2210-9838. Disponível em: <http://www.sciencedirect.com/science/article/pii/S2210983815000851>.

MEDEIROS, M. A. F. The nonlinear evolution of a wavetrain emanating from a point source in a boundary layer. J. Fluid Mech., v. 508, p. 287-317, 2004.

MEDEIROS, M. A. F.; GASTER, M. The influence of phase on the nonlinear evolution of wavepackets in boundary layers. J. Fluid Mech., v. 397, p. 259-283, 1999.

The production of sub-harmonic waves in the nonlinear evolution of wavepackets in boundary layers. J. Fluid Mech., v. 399, p. 301-318, 1999.

MOIN, P.; MAHESH, K. Direct numerical simulation: a tool in turbulence research. Annual review of fluid mechanics, Annual Reviews 4139 El Camino Way, PO Box 10139, Palo Alto, CA 94303-0139, USA, v. 30, n. 1, p. 539-578, 1998.

NG, L. L.; ERLEBACHER, G. Secondary instabilities in compressible boundary layers. Physics of Fluids A, v. 4, n. 4, p. 710-726, 1992. Disponível em: $<$ http://scitation.aip.org/content/aip/journal/pofa/4/4/10.1063/1.858290>. 
NORMAND, X.; LESIEUR, M. Direct and large-eddy simulations of transition in the compressible boundary layer. Theoretical and Computational Fluid Dynamics, v. 3, n. 4, p. 231-252, 1992. ISSN 1432-2250. Disponível em: <http://dx.doi.org/10$.1007 / \mathrm{BF} 00417915>$.

OPFER, H. Active cancellation of 3D Tollmien-Schlichting waves in the presence of sound and vibrations. Tese (Doutorado) - DLR, 2002.

ÖZGEN, S.; KIRCALI, S. A. Linear stability analysis in compressible, flat-plate boundary-layers. Theoretical and Computational Fluid Dynamics, v. 22, n. 1, p. 1-20, 2007. ISSN 1432-2250. Disponível em: <http://dx.doi.org/10.1007/s00162-0070071-0>.

PAULA, I. B. de et al. Weakly nonlinear stages of boundary-layer transition initiated by modulated tollmien schlichting waves. Journal of Fluid Mechanics, v. 732, p. 571-615, 10 2013. ISSN 1469-7645. Disponível em: <http://journals.cambridge.org/article_S0022112013004205>.

PELTZER, I. Comparative in-flight and wind tunnel investigation of the development of natural and controlled disturbances in the laminar boundary layer of an airfoil. Experiments in Fluids, Springer, v. 44, n. 6, p. 961-972, 2008.

PIROZZOLI, S.; BERNARDINI, M.; GRASSO, F. Direct numerical simulation of transonic shock/boundary layer interaction under conditions of incipient separation.

Journal of Fluid Mechanics, v. 657, p. 361-393, 8 2010. ISSN 1469-7645. Disponível em: <http://journals.cambridge.org/article_S0022112010001710>.

POINSOT, T. J.; LELE, S. K. Boundary conditions for direct simulations of compressible viscous flows. Journal of Computational Physics, v. 101, p. 104-129, 1992.

REEH, A. D.; TROPEA, C. Behaviour of a natural laminar flow aerofoil in flight through atmospheric turbulence. Journal of Fluid Mechanics, Cambridge University Press, Cambridge, UK, v. 767, p. 394-429, 003 2015. Disponível em: <https://www.cambridge.org/core/article/behaviour-of-a-natural-laminar-flow-aerofoil-in-flightthrough-atmospheric-turbulence/C3AA476CF8B74769B9CDD20EE623ECAE>.

REYNOLDS, O. On the experimental investigation of the circumstances which determine whether the motion of water shall be direct or sinuous, and the law of resistance in parallel channels. Phil. Trans. Roy. Soc., v. 174, p. 935-982, 1883.

RICCO, P.; TRAN, D.-L.; YE, G. Wall heat transfer effects on klebanoff modes and tollmien schlichting waves in a compressible boundary layer. Physics of Fluids, v. 21, n. 2, 2009. Disponível em: <http://scitation.aip.org/content/aip/journal/pof2/21/2/10$.1063 / 1.3054155>$.

RICCO, P.; WU, X. Response of a compressible laminar boundary layer to free-stream vortical disturbances. Journal of Fluid Mechanics, v. 587, p. 97-138, 9 2007. ISSN 1469-7645. Disponível em: <http://journals.cambridge.org/article_S0022112007007070>.

RIST, U.; FASEL, H. Direct numerical simulation of controlled transition in a flat plate boundary layer. J. Fluid Mech., v. 298, p. 211-248, 1995. 
SAYADI, T.; HAMMAN, C. W.; MOIN, P. Direct numerical simulation of complete h-type and k-type transitions with implications for the dynamics of turbulent boundary layers. Journal of Fluid Mechanics, v. 724, p. 480-509, 6 2013. ISSN 1469-7645. Disponível em: <http://journals.cambridge.org/article_S0022112013001420>.

SCHLICHTING, H. Zur Entstehung der Turbulenz bei der Plattenströmung. In: Math. Phys. Klasse. [S.l.]: Mitt. Nachr. Ges. Wiss. Göttingen, 1933. p. 182-208.

SCHLICHTING, H.; GERSTEN, K. Boundary-layer theory. [S.l.]: Springer Science \& Business Media, 2003.

SCHMID, P.; HENNINGSON, D. S. A new mechanism for rapid transition involving a pair of oblique waves. Phys. Fluids A, v. 9, p. 1986-1989, 1992.

SCHMID, P. J.; HENNINGSON, D. S. Stability and transition in shear flows. [S.l.]: Springer-Verlag, 2001.

SCHMID, P. J.; REDDY, S. C.; HENNINGSON, D. S. Transition thresholds in boundary layer and channel flows. In: ___ Advances in Turbulence VI: Proceedings of the Sixth European Turbulence Conference, held in Lausanne, Switzerland, 2-5 July 1996. Dordrecht: Springer Netherlands, 1996. p. 381-384. ISBN 978-94-009-0297-8. Disponível em: <http://dx.doi.org/10.1007/978-94-009-0297-8_108>.

SCHUBAUER, G. B.; SKRAMSTAD, H. K. Laminar boundary layer oscillations and transition on a flat plate. [S.l.], 1943.

SEMIONOV, N. V.; KOSINOV, A. D.; MASLOV, A. A. Transition control of supersonic boundary layer on flat plate. In: IUTAM Symposium on Mechanics of Passive and Active Flow Control: Proceedings of the IUTAM Symposium held in Göttingen, Germany, 7-11 September 1998. Dordrecht: Springer Netherlands, 1999. p. 323-328. ISBN 978-94-011-4199-4. Disponível em: <http://dx.doi.org/10.1007/978-94-011-4199-4_52>.

SENGUPTA, T.; DIPANKAR, A.; RAO, A. K. A new compact scheme for parallel computing using domain decomposition. Journal of Computational Physics, v. 220, n. 2 , p. $654-677,2007$. ISSN 0021-9991.

SEVERIN, M. Direct Numerical Simulations Of Three-Dimensional Wavepackets In A Flat-Plate Boundary-Layer. Dissertação (Mestrado) University of Arizona, 2012.

SHAIKH, F. N. Investigation of transition to turbulence using white noise excitation and local analysis techniques. J. Fluid Mech., v. 348, p. 29-83, 1997.

SINGER, B.; FERZIGER, J.; REED, H. Investigation of the effects of initial disturbances on plane channel transition. In: [S.1.]: American Institute of Aeronautics and Astronautics, 1986. (Aerospace Sciences Meetings). Doi:10.2514/6.1986-433.

SIVASUBRAMANIAN, J.; FASEL, H. F. Numerical investigation of the development of three-dimensional wavepackets in a sharp cone boundary layer at mach 6 . Journal of Fluid Mechanics, v. 756, p. 600-649, 10 2014. ISSN 1469-7645. Disponível em: <http://journals.cambridge.org/article_S0022112014004340>. 
Direct numerical simulation of transition in a sharp cone boundary layer at mach 6 : fundamental breakdown. Journal of Fluid Mechanics, v. 768, p. 175-218, 4 2015. ISSN 1469-7645. Disponível em: <http://journals.cambridge.org/article_S0022112014006788>.

SPALART, P. R.; YANG, K.-S. Numerical study of ribbon-induced transition in Blasius flow. J. Fluid Mech., v. 178, p. 345-365, 1987.

THIBERT, J.; ARNAL, D. A review of \{ONERA $\}$ aerodynamic research in support of a future supersonic transport aircraft. Progress in Aerospace Sciences, v. 36, n. 8, p. 581 - 627, 2000. ISSN 0376-0421. Disponível em: <http://www.sciencedirect.com/science/article/pii/S0376042100000105>.

THUMM, A.; WOLZ, W.; FASEL, H. Laminar-turbulent transition: Iutam symposium toulouse/france september 11-15, 1989. In: Berlin, Heidelberg: Springer

Berlin Heidelberg, 1990. cap. Numerical Simulation of Spatially Growing ThreeDimensional Disturbance Waves in Compressible Boundary Layers, p. 303-308. ISBN 978-3-642-84103-3. Disponível em: <http://dx.doi.org/10.1007/978-3-642-84103-3_27>.

TOLLMIEN, W. über die Entstehung der Turbulenz. In: Math. Phys. Klasse. [S.l.]: Mitt. Nachr. Ges. Wiss. Göttingen, 1929. p. 21-44.

TUMIN, A. Three-dimensional spatial normal modes in compressible boundary layers.

Journal of Fluid Mechanics, v. 586, p. 295-322, 9 2007. ISSN 1469-7645. Disponível em: <http://journals.cambridge.org/article_S002211200700691X>.

TUNNEY, A. P. et al. A new inviscid mode of instability in compressible boundary-layer flows. Journal of Fluid Mechanics, v. 785, p. 301-323, 12 2015. ISSN 1469-7645.

Disponível em: <http://journals.cambridge.org/article_S0022112015006278>.

VASUDEVA, B. R. Boundary-layer instability experiment with localized disturbance. Journal of Fluid Mechanics, v. 29, p. 745-763, 9 1967. ISSN 1469-7645.

VISBAL, M. R.; GAITONDE, D. V. On the use of higher-order finite-difference schemes on curvilinear and deforming meshes. Journal of Computational Physics, v. 181, n. 1, p. 155 - 185, 2002. ISSN 0021-9991. Disponível em: <http://www.sciencedirect.com/science/article/pii/S0021999102971172>.

WASISTHO, B.; GEURTS, B.; KUERTEN, J. Simulation techniques for spatially evolving instabilities in compressible flow over a flat plate. Computers and Fluids, v. 26, n. 7, p. 713 - 739, 1997. ISSN 0045-7930. Disponível em: <http://www.sciencedirect.com/science/article/pii/S0045793097000212>.

WHITE, F. Vicous Fluid Flow. [S.l.]: McGraw-Hill, 1974.

WU, X.; STEWART, P. A. Interaction of phase-locked modes: a new mechanism for the rapid growth of three-dimensional disturbances. Journal of Fluid Mechanics, Cambridge Univ Press, v. 316, p. 335-372, 1996.

WU, X.; STEWART, P. A.; COWLEY, S. J. On the catalytic role of the phase locked interaction of tollmien schlichting waves in boundary-layer transition. J. Fluid Mech., v. 590, p. $265-294,2007$. 
YANG, K.-S.; SPALART, P. R.; FERZIGER, J. H. Numerical studies of natural transition in a decelerating boundary layer. Journal of Fluid Mechanics, v. 240, p. 433-468, 7 1992. ISSN 1469-7645. Disponível em: <http://journals.cambridge.org/article_S0022112092000156>.

YEO, K. S. et al. DNS of wavepacket evolution in a Blasius boundary layer. J. Fluid Mech., v. 652, p. 333-372, 2010.

YONGSHENG, L.; WE, S. Laminar - Turbulent Transition of a Low Reynolds Number Rigid or Flexible Airfoil. AIAA Journal, American Institute of Aeronautics and Astronautics, v. 45, n. 7, p. 1501-1513, 2007. ISSN 0001-1452. Doi: 10.2514/1.25812. 